\title{
The Aqueous Environment: Lessons From Small Molecules
}

\author{
Lorna Dougan
}

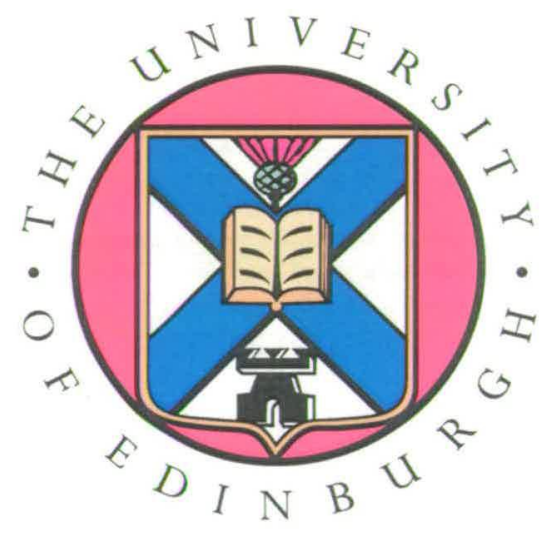

Thesis submitted for the degree of Doctor of Philosophy

The University of Edinburgh

2005 


\section{Abstract}

Amphiphiles are a particularly important class of molecule containing both hydrophobic and hydrophilic domains with competing solubility properties. The simplest amphiphiles are the primary alcohols, such as methanol, ethanol and propanol. Aqueous solutions of these simple molecules are widely studied and have been found to be completely miscible in all proportions and at all state points studied. Recently, experimental and computational studies on aqueous methanol have revealed unexpectedly complex behaviour at medium length-scales leading to a substantially revised view of miscibility in this prototype aqueous amphiphile. In particular, molecular-level segre-

gation has been observed with structural details consistent with those expected for a hydrophobically-driven system.

An emerging route toward the development, testing and refinement of detailed molecular models of the hydrophobic interaction, hydration and the physics of aqueous macromolecules involves the use of small molecule systems as 'prototypes'. These models are potentially of wider significance to areas such as membrane and protein stability. Despite the intense activity and success in studying these model systems at room temperature and pressure, there have been no systematic investigations aimed at mapping out the behaviour of these observed extended structures under non-ambient conditions. In this thesis the simple, prototype system of methanol and water has been studied. High resolution picosecond fluorescence spectroscopy provides an insight into the dynamics of methanol-water solutions over a range of concentrations. In order to an- 
swer fundamental physical questions about the molecular and bulk properties of the methanol-water system, work was carried out for the first time in the low temperature and supercooled regime revealing very interesting results.

An extensive series of neutron diffraction experiments combined with Empirical Structural Refinement Analysis (EPSR) has shown that mixtures of methanol and water exhibit extended structures in solution despite the components being fully miscible in all proportions. Of particular interest is a concentration region (methanol mole fraction between 0.27 and 0.54 ) where both methanol and water appear to form separate, percolating networks. This is the concentration range where many transport properties and thermodynamic excess functions reach extremal values. The observed concentration dependence of several of these material properties of the solution may therefore have a structural origin.

Molecular segregation in methanol-water mixtures is studied across a wide concentration range as a function of temperature and pressure. Cluster distributions obtained from neutron diffraction point to significantly enhanced segregation as the mixtures are cooled or compressed. The observed behaviour is consistent with an approach to an upper critical solution point. Such a point would appear to be 'hidden' below the freezing line, thereby precluding observation of the two-fluid region.

Finally, it is shown that the negative excess entropy of mixing characteristic of aqueous lower alcohols can be understood quantitatively in terms of molecular-scale segregation of the components. A simple model is presented and used to understand the behaviour of methanol-water solutions under extreme conditions using molecular clustering data from neutron diffraction and obviates the need to invoke other restructuring concepts which, though well-known, are unsupported by recent experiments. 


\section{Declaration}

This thesis has been composed by myself and has not been submitted in any previous application for a degree. The work reported within was performed by myself, unless otherwise stated.

Lorna Dougan 


\section{Acknowledgements}

I would like to thank my supervisor Jason Crain for all his support and guidance over the last three years. I am very grateful to Alan Soper for his huge enthusiasm and taking the time to share his superb breadth of knowledge on all things liquid.

Thanks to John Finney, Simon Bates and Rowan Hargreaves for their many helpful discussions. Steven Magennis for his help and advice during the fluorescence spectroscopy experiments and Hugh Vass for excellent technical assistance during the low temperature fluorescence work.

My wonderful friends for their never-fading encouragement, support and fun! This includes many hours of dancing in 'Opium', running the KB4, many, many hours over coffee and cake and many weekends in the hills, 'Munro bagging'. Thanks also for just listening when it all got too much!

Thank-you to Paul for keeping my sanity intact! For keeping a smile on my face and making sure I had some fun during the ordeal of 'writing-up'.

Finally, a huge thank-you to mum, dad, John, Richard and Rachel for being there and showing me, through their own achievements and endeavours, that difficult targets and challenges can be met. 


\section{Contents}

Abstract

Declaration $\quad$ v

Acknowledgements vii

1 Introduction 1

1.1 Crucial Role of Water in Biology . . . . . . . . . . . . . . 1

1.2 A Model System . . . . . . . . . . . . . . . 6

1.3 Exploring Aqueous Alcohol Solutions . . . . . . . . . . . . 7

1.4 Thesis Layout . . . . . . . . . . . . . . . . . . . . . . . . . 8

2 Background Theory 11

2.1 Water- A Very Important Substance . . . . . . . . . . . . . . . 11

2.2 Structure in Liquids . . . . . . . . . . . . . . . . . . . 15

2.3 The Aqueous Environment . . . . . . . . . . . . . . . . 17

2.3.1 Polar and Non-Polar Liquids . . . . . . . . . . . . . . . . 17

xxix 
2.3.2 Amphiphiles ..................... 18

2.3.3 The Hydrophobic Interaction . . . . . . . . . . . . . . . . . . 19

2.4 Thermodynamic Properties of Liquid Mixtures . . . . . . . . . . . . 23

2.4 .1 Gibbs Free Energy . . . . . . . . . . . . . . . . 23

2.4 .2 Excess Functions . . . . . . . . . . . . . . . . . . . . . . 25

2.4 .3 Miscibility and Immiscibility . . . . . . . . . . . . . . . 26

2.5 Review of Current Understanding . . . . . . . . . . . . . . 28

2.5.1 Neutron Diffraction of Aqueous Alcohols . . . . . . . . . 28

2.5.2 Computer Simulation . . . . . . . . . . . . . 32

3 Probing Structure \& Dynamics 37

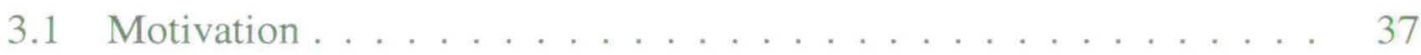

3.2 Principles of Fluorescence . . . . . . . . . . . . . . . . 37

3.3 Fluorescence Spectroscopy Observables . . . . . . . . . . . . . . 41

3.3.1 Excitation Process and Emission Process . . . . . . . . . 41

3.3.2 Fluorescence Intensity Decay and Lifetime . . . . . . . . . . 42

3.4 Experimental Procedure . . . . . . . . . . . . . . . . . 44

3.4.1 The Experimental Probe . . . . . . . . . . . . . . . . . . . 44

3.4.2 Sample Preparation . . . . . . . . . . . . . . . 45

3.4.3 Absorption and Emission Measurements . . . . . . . . . . . 46

3.4.4 Time Resolved Fluorescence Measurements . . . . . . . . . . 47

3.4.5 Analysis of Fluorescence Intensity Decays . . . . . . . . . 49 
3.4.6 Low Temperature Experimental Setup . . . . . . . . . . . 52

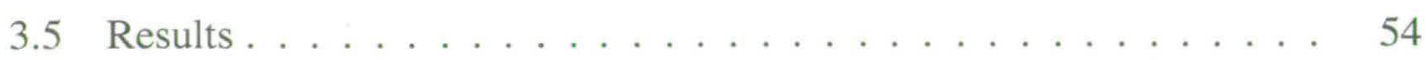

3.5.1 Behaviour of the Probe ............... 54

3.5.2 Structure of the System . . . . . . . . . . . . 56

3.5.3 Dynamics of the System . . . . . . . . . . . . . 60

3.6 Discussion ............................ 66

4 Probing the Structure of Liquids $\quad 73$

4.1 Neutron Diffraction . . . . . . . . . . . . . . 73

4.1.1 The Diffraction Experiment . . . . . . . . . . 75

4.1.2 Neutron Diffraction Under Non-Ambient Conditions . . . . . 76

4.2 From Scattered Intensity to Structure Factor . . . . . . . . . . . . 76

4.2.1 The Scattering Amplitude . . . . . . . . . . . . . . . 79

4.2.2 The Scattered Intensity . . . . . . . . . . . . . . 80

4.2.3 The Structure Factor . . . . . . . . . . . . . . 82

4.3 Real Liquid Systems . . . . . . . . . . . . . . . . . . . . 83

4.3.1 Coherent and Incoherent Scattering . . . . . . . . . . . 84

4.3.2 Inelastic Scattering Considerations . . . . . . . . . . . . 86

4.4 Isotope Substitution Explained . . . . . . . . . . . . . . 87

4.4.1 Isotope Substitution Example . . . . . . . . . . . . . 88

4.5 Correcting the Data . . . . . . . . . . . . . . 91

4.6 Computational Modelling Technique . . . . . . . . . . . . . . . 94 
4.6.1 Fundamentals of EPSR . . . . . . . . . . . . . . . . . . 96

4.6.2 Defining the Reference Interatomic Potential . . . . . . . . . 99

4.6.3 Running the Simulation . . . . . . . . . . . . . . . 100

4.6.4 The Potential of Mean Force . . . . . . . . . . . . . . . . . . 100

4.6.5 Summary of EPSR . . . . . . . . . . . . . . . 102

5 Bi-Percolating Liquid Mixture $\quad 103$

5.1 Motivation . . . . . . . . . . . . . . . . . 103

5.2 Experimental Details . . . . . . . . . . . . . . . . . . . . 104

5.3 Structure Factor Fits . . . . . . . . . . . . . . . 106

5.4 Micro-segregation . . . . . . . . . . . . . . . . . 106

5.5 Defining the Clusters . . . . . . . . . . . . . . . . 108

5.6 Percolation Theory . . . . . . . . . . . . . . . . . . . . . . 109

5.7 A Bi-Percolating Liquid Mixture . . . . . . . . . . . . . . . . . . . . 112

5.8 Discussion . . . . . . . . . . . . . . . . . . . . 115

6 Cooling Enhances Segregation 121

6.1 Motivation . . . . . . . . . . . . . . . . 121

6.2 Experimental Details . . . . . . . . . . . . . . . . . . . 122

6.3 Structure Factor Fits . . . . . . . . . . . . . . . . . . . . . . . . 123

6.4 Cooling Enhances Micro-Segregation . . . . . . . . . . . . . . . . 125

6.5 Enhanced Hydrogen Bond Interactions . . . . . . . . . . . . . . . . . 132

6.6 Cooling Enhances Hydrophobic Interaction . . . . . . . . . . . . . 142 
6.7 Methanol-Water Correlations . . . . . . . . . . . . . . 146

6.8 Discussion . . . . . . . . . . . . . . . . . . . . . . 148

7 Compression Enhances Segregation 153

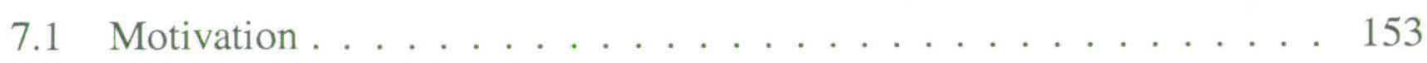

7.2 Experimental Details . . . . . . . . . . . . . . . 154

7.3 Structure Factor Fits . . . . . . . . . . . . . . . 156

7.4 Compression Enhances Micro-segregation . . . . . . . . . . . . . 156

7.5 The Hydrophobic Effect Under Pressure . . . . . . . . . . . . . . . 159

7.6 Water Under Pressure . . . . . . . . . . . . . . . . . . 161

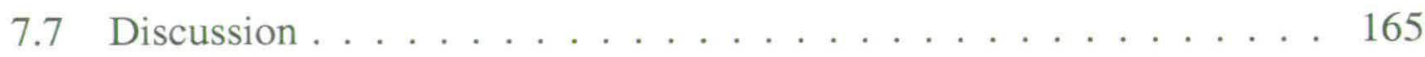

8 A Simple Model to Explain Observed Entropy 169

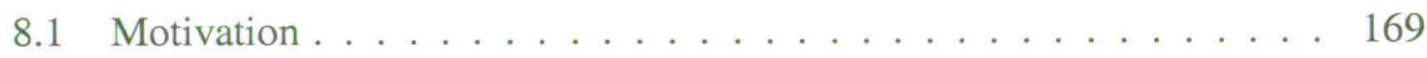

8.2 Calculating the Excess Entropy . . . . . . . . . . . . . 170

8.3 Excess Entropy of Methanol-Water Mixtures . . . . . . . . . . . . 176

8.4 Influence of Cooling on Excess Entropy . . . . . . . . . . . . . . 178

8.5 Influence of Compression on Excess Entropy . . . . . . . . . . . . . 180

8.6 Summary and Discussion . . . . . . . . . . . . . . 181

9 Conclusions and Future Work $\quad 187$

9.1 Conclusions .......................... 187

9.2 Future Work . . . . . . . . . . . . . . . . . . . . 188 


\section{List of Figures}

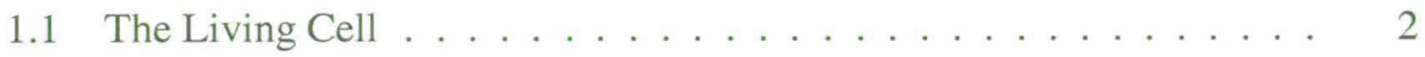

1.2 Illustration of a cartoon protein containing hydrophobic (black circles) and hydrophilic (white circles) regions. In aqueous solution the hydrophobic regions are buried in the core, shielded by the hydrophilic regions from surrounding water. . . . . . . . . . . . . . . 3

1.3 Intermediate structures in the self assembly of the small peptide KFE8 in aqueous solution. The images are Atomic Force Microscopy scans at different times after preparation of solution (a) after 8 minutes, (b) 35 minutes, (c) 2 hours and (d) 30 hours[5]. . . . . . . . . . . . .

2.1 Illustration of a water molecule showing the oxygen atom, $O$, with 2 lone pairs of electrons (shown as small red circles) and consequently a partial -ve charge and the two hydrogen atoms $H$ with a partial +ve

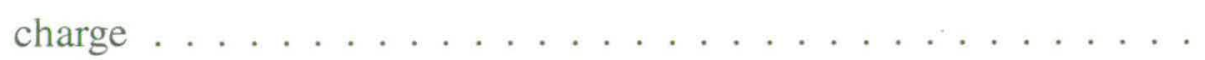

2.2 Illustration of hydrogen bonded network with each water molecule participating in 4 hydrogen bonds. Each hydrogen acting as a donor for a hydrogen bond and oxygen acting as an acceptor for hydrogen bonds. Hydrogen bonds are shown as blue dashed lines, while covalent bonds are shown as black lines. 
2.3 Schematic of a typical radial distribution function for a liquid showing the first and second coordination shell (blue lines) . . . . . . . . . . 16

2.4 Cartoons illustrating a phospholipid with polar hydrophilic head and non-polar hydrophobic tail (left) and the lipid bilayer formed (right) .

2.5 Clathrate Cage of Water Surrounding Hydrophobic Methane Molecule[25] 21

2.6 Illustration of hydrophobic hydration (left) and hydrophobic interaction (right) where the blue crosses represent water and the red circles represent a hydrophobic species . . . . . . . . . . . . . .

2.7 Schematic of miscible and immiscibility regions for a binary mixture with (a) Upper Critical Solution Temperature (UCST), (b)Lower Critical Solution Temperature (LCST) and (c) Closed Loop Immiscibility Region.

2.8 The hydrogen-hydrogen correlation function for a methanol-water $x=$ 0.10 mole fraction solution (line) compared to the same function for pure water (circles). The intramolecular peak at $\mathrm{r} \sim 1.5 \AA$, the hydrogen bond peak at $\mathrm{r} \sim 2.3 \AA$, and a third characteristic peak near $\mathrm{r} \sim 3.8$ $\AA$ are all clearly visible in both cases[26] . . . . . . . . . . . . . . .

2.9 Snapshot of micro-segregation in a methanol-water $x=0.05$ mole fraction solution obtained from neutron diffraction and Empirical Potential Structural Refinement technique[13]. Water and methanol oxygens are shown as white atoms. The methanol carbon atom is shown in black. Black bonds indicate methanol carbons in the first coordination shell. White bonds join all oxygen atoms. 
2.10 Micro-segregation in a methanol-water $x=0.70$ mole fraction system[12]. Methyl groups are shown as grey spheres. Large yellow spheres have been used to highlight the positions of water molecules and small red spheres denote methanol oxygen atoms. Yellow bonds join water oxygen atoms to other oxygen atoms within their first coordination shell. .

2.11 Optimised dilute methanol system, 1 methanol molecule and 12 water molecules. (a)The full cluster and (b)fragment containing only the central methanol molecule and the water molecules hydrogen bonded to the central methanol molecule, where — denotes hydrogen bonds[42] 36

3.1 The Jablonski diagram showing the ground state $S_{0}$, first excited state $S_{1}$, second excited state $S_{2}$ and triplet excited state $T_{1} \ldots \ldots$

3.2 The Frank Condon energy diagram . . . . . . . . . . . . . . 40

3.3 Illustration of Fluorescence Intensity Decay, showing fluorescence intensity decay as a function of fluorescence intensity with time . . . . .

3.4 Molecular structure of 1 - anilinonaphthalene -8 - sulfonic acid (ANS). The fluorescence probe . . . . . . . . . . . . . .

3.5 Schematic of Fluorescence Spectrometer Set-up on Optical Bench .

3.6 Time Correlated Single Photon Counting . . . . . . . . . . . . .

3.7 Illustration of Exponential fit (blue line) to a Fluorescence Intensity Decay (green line) of a fluorophore in solution and residuals (red line) indicating the goodness of fit of data. . . . . . . . . . . 52

3.8 Time Resolved Fluorescence Intensity Decays for ANS in water (left) and ANS in methanol (right) . . . . . . . . . . . . . . 
3.9 The fluorescence lifetime of 1,8-anilinonaphthalene sulfonate ANS in methanol-water mixtures, ethanol-water mixtures and acetonitrile mixtures[54] in relation to concentration of solvent in mole fraction . . . . . . . 58

3.10 The time resolved fluorescence decay of 1,8-anilinonaphthalene sulfonate ANS in methanol-water mixture $x=0.50$ mole fraction at $298 \mathrm{~K}$. The green line shows the fluorescence intensity decay, the black line shows the instrument response function, the blue line shows the exponential fit to the data and the red line shows the residuals, indicating the goodness of fit to the data. . . . . . . . . . . . . . . 59

3.11 Temperature dependence of ANS Fluorescence Decay times in Water $\left(\lambda_{e m}=\right.$ $520 \mathrm{~nm}), \operatorname{Methanol}\left(\lambda_{e m}=490 \mathrm{~nm}\right)$, or Methanol-Water $x=0.5,\left(\lambda_{e m}=\right.$

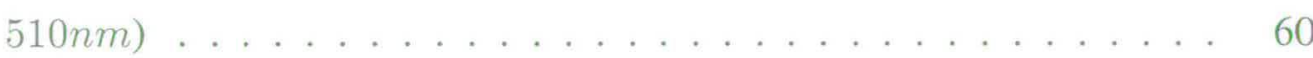

3.12 Wavelength dependence of the amplitudes of $\tau_{1}$ and $\tau_{2}$ for the biexponential fit of the fluorescence decay of ANS in methanol at 200 $\mathrm{K}$

3.13 The time resolved fluorescence intensity decay of 1,8-anilinonaphthalene sulfonate ANS in methanol-water mixture $x=0.50$ mole fraction at $200 \mathrm{~K}$. The green line shows the fluorescence intensity decay, the black line shows the instrument response function, the blue line shows the exponential fit to the data and the red line shows the residuals, indicating the goodness of fit to the data.

3.14 Wavelength dependence of the amplitudes of $\tau_{1}$ and $\tau_{2}$ for the biexponential fit of the fluorescence decay of ANS in methanol-water $x=0.50$ mole fraction at $200 \mathrm{~K}$ 
3.15 A simple schematic representation of solvent relaxation. $S_{0}$ represents the equilibrium ground state of the ANS molecule, $S_{1}^{\prime}$ and $S_{1}^{\prime \prime}$ the nonequilibrium excited states, $S_{1}$ the equilibrium excited state and $S_{0}^{\prime}$ the non-equilibrium ground state. The vertical blue line represents absorption, the vertical green line represents emission and the dashed vertical red line represents solvent relaxation. . . . . . . . . . . . .

4.1 The diffraction experiment .................. 75

4.2 Cut away view showing the interior of the Ti-Zr sample can[79] . . .

4.3 Schematic view of the pressure handling system. V1, V2, V3 and V4 are valves, $\mathrm{S}$ is a syringe used to fill up the system with water after evacuation, $\mathrm{RV}$ is a release valve (100 bar), $\mathrm{N}$ is a pressure generator, $\mathrm{BD}$ are two bursting discs (5000 bar) and PG is the pressure gauge[79]

4.4 Illustration of the geometry of a scattering experiment with incident wave vector $k$ and scattered wave vector $k^{\prime}$. The solid angle $\mathrm{d} \Omega$ is defined about a specific direction $\theta$ and a detector collects particles scattered in the direction defined by the angle $\theta \ldots 78$

4.5 Schematic of a flat plate Titanium-Zirconium sample can . . . . . . 92

4.6 Schematic of the detectors on the SANDALS instrument at the ISIS facility, Rutherford Appleton Laboratory, UK . . . . . . . . . . 93

4.7 A flowchart illustrating the steps involved in the data correction procedure for a neutron diffraction experiment on the SANDALS instrument

4.8 Flowchart illustrating the main steps involved in the Empirical Potential Structural Refinement(EPSR) . . . . . . . . . . . . . .

4.9 Flowchart illustrating the main steps involved in the Empirical Potential Structural Refinement (EPSR) . . . . . . . . . . . . . . 
5.1 Typical example of the fits (lines) obtained by the EPSR computer simulation procedure compared to the original data (circles). The data shown in this case $(x=0.54)$ are the interference differential scattering cross-sections for the samples (i) through (vii) described under the Experimental Details section . . . . . . . . . . . . . . . . . 107

5.2 Snapshot of an experimentally-constrained EPSR model of the methanolwater mixture at $x=0.54$ showing clusters of the segregated components. Methyl groups are shown as black spheres, large yellow spheres highlight the position of water molecules and small red spheres denote methanol oxygen atoms.

5.3 Illustration of a square lattice with some occupied sites (left) leading to some clusters through nearest neighbour sites (right) . . . . . . . . 110

5.4 Illustration of systems with increasing probability of site occupation (left to right) with a fully percolating cluster spanning the dimensions

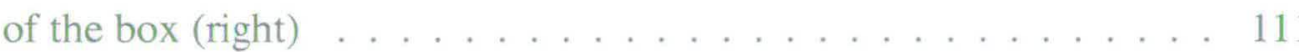

5.5 Schematic of cluster size distribution showing the cluster size (number of molecules) as a function of cluster size distribution. Also shown is the predicted power law $n_{s} \approx s^{-2.2}$ for random percolation on a 3 -d cubic lattice[92].

5.6 Cluster size distributions for water (right) and methanol (left) clusters in methanol-water mixtures mole fractions $0.05,0.27,0.54$ and 0.7 . The dashed lines show the predicted cluster size distribution at the percolation threshold[92]. Percolation in the simulated box occurs when clusters of a size close to the number of molecules in the simulation box form (vertical lines on the right hand side of the plot). 
5.7 Excess entropies of mixing of alcohols with water at $25^{\circ}$ (left), Heats of mixing of alcohols with water at $25^{\circ}$ (middle), Excess free energies of mixing of alcohols with water at $25^{\circ}$ (right)[71] . . . . . . . . 11

5.8 Ratio of number of methanol molecules at the surface of a cluster to total number of methanol molecules in a cluster (left). Ratio of number of water molecules at the surface of a cluster (as defined by being hydrogen bonded to a methanol hydroxyl group) to total number of water molecules in a cluster (right). Dashed line shows the $N^{-1 / 3}$ behaviour expected if the clusters grew equally in 3 dimensions with $\mathrm{N}$ the number of molecules in a cluster. . . . . . . . . . . . . . . 118

6.1 Composite partial structure factors fits (red lines) obtained by the EPSR computer simulation procedure compared to the original data (black circles) for methanol-water mixture mole fraction $\mathrm{x}=0.27$ at $298 \mathrm{~K}$ (upper) and $\mathrm{x}=0.27$ at $238 \mathrm{~K}$ (lower) . . . . . . . . . . . .

6.2 Composite partial structure factors fits (red lines) obtained by the EPSR computer simulation procedure compared to the original data (black circles) for methanol-water mixture mole fraction $\mathrm{x}=0.54$ at $298 \mathrm{~K}$ (top), $\mathrm{x}=0.54$ at $260 \mathrm{~K}$ (middle) and $\mathrm{x}=0.54$ at $190 \mathrm{~K}$ (bottom) . . 128

6.3 Water cluster size distributions in two different methanol-water mixtures, mole fractions $x=0.27$ (left) and $x=0.54$ (right). The black line indicates the system at ambient temperature while the red line indicates the cooled system. The dashed lines show the predicted cluster size distribution at the percolation threshold[92] . . . . . . . . . 
6.4 Methanol cluster size distributions in two different methanol-water mixtures, mole fractions 0.27 (left) and 0.54 (right). The black line indicates the system at ambient temperature while the red line indicates the cooled system. The dashed lines show the predicted cluster size distribution at the percolation threshold $[92] \ldots \ldots . . . .$.

6.5 Snapshot of an experimentally-constrained EPSR model of the methanolwater mixture at $x=0.54$ at $298 \mathrm{~K}$ (left) and $190 \mathrm{~K}$ (right) showing clusters of the segregated components. Methyl groups are shown as red spheres, large blue spheres highlight the position of water molecules and small grey spheres denote methanol oxygen atoms. . . . . . . . 130

6.6 Snapshot of an experimentally-constrained EPSR model of the methanolwater mixture at $x=0.54$ at $298 \mathrm{~K}$ (left) and $190 \mathrm{~K}$ (right) were only the water molecules are shown, highlighted as large blue spheres (water oxygen) and small white spheres (water hydrogens). Hydrogen bonding between water molcules is illustrated with a dashed red line

6.7 Top panel shows partial radial distribution function for OHw correlations in a methanol-water $x=0.27$ mole fraction mixture (left) at 293 $\mathrm{K}$ (black solid line) and mixture at $238 \mathrm{~K}$ (black dashed line) and for a methanol-water $\mathrm{x}=0.54$ mole fraction mixture (right) at $298 \mathrm{~K}$ (black solid line), mixture at $260 \mathrm{~K}$ (black dashed line) and mixture at $190 \mathrm{~K}$ (black dotted line). Lower panel shows partial radial distribution function for HOw correlations in a methanol-water $\mathrm{x}=0.27$ mole fraction mixture (left) at $293 \mathrm{~K}$ (black solid line) and mixture at $238 \mathrm{~K}$ (black dashed line) and for a methanol-water $\mathrm{x}=0.54$ mole fraction mixture (right) at $298 \mathrm{~K}$ (black solid line), mixture at $260 \mathrm{~K}$ (black dashed line) and mixture at $190 \mathrm{~K}$ (black dotted line) . . . . . . . . . . . . . 133 
6.8 Partial radial distribution function for OOw correlations in a methanolwater $\mathrm{x}=0.27$ mole fraction mixture (left) at $293 \mathrm{~K}$ (black solid line) and mixture at $238 \mathrm{~K}$ (black dashed line) and for a methanol-water $\mathrm{x}=$ 0.54 mole fraction mixture (right) at $298 \mathrm{~K}$ (black solid line), mixture at $260 \mathrm{~K}$ (black dashed line) and mixture at $190 \mathrm{~K}$ (black dotted line).

6.9 Partial radial distribution function for $\mathrm{OH}$ correlations in a methanolwater $\mathrm{x}=0.27$ mole fraction mixture (left) at $293 \mathrm{~K}$ (black solid line) and mixture at $238 \mathrm{~K}$ (black dashed line) and for a methanol-water $\mathrm{x}=$ 0.54 mole fraction mixture (right) at $298 \mathrm{~K}$ (black solid line), mixture at $260 \mathrm{~K}$ (black dashed line) and mixture at $190 \mathrm{~K}$ (black dotted line) In both $\mathrm{g}(\mathrm{r}) \mathrm{s}$ the distribution is also shown for pure methanol (red line) for comparison.

6.10 Partial radial distribution function for OO correlations in a methanolwater $\mathrm{x}=0.27$ mole fraction mixture (left) at $293 \mathrm{~K}$ (black solid line) and mixture at $238 \mathrm{~K}$ (black dashed line) and for a methanol-water $\mathrm{x}=$ 0.54 mole fraction mixture (right) at $298 \mathrm{~K}$ (black solid line), mixture at $260 \mathrm{~K}$ (black dashed line) and mixture at $190 \mathrm{~K}$ (black dotted line) In both RDF's the distribution is also shown for pure water (red line) for comparison.

6.11 Partial radial distribution function for $\mathrm{HH}$ correlations in a methanolwater $\mathrm{x}=0.27$ mole fraction mixture (left) at $293 \mathrm{~K}$ (black solid line) and mixture at $238 \mathrm{~K}$ (black dashed line) and for a methanol-water $\mathrm{x}=$ 0.54 mole fraction mixture (right) at $298 \mathrm{~K}$ (black solid line), mixture at $260 \mathrm{~K}$ (black dashed line) and mixture at $190 \mathrm{~K}$ (black dotted line) In both RDF's the distribution is also shown for pure water (red line) for comparison. 
6.12 Partial radial distribution function for OwHw correlations in a methanolwater $\mathrm{x}=0.27$ mole fraction mixture (left) at $293 \mathrm{~K}$ (black solid line) and mixture at $238 \mathrm{~K}$ (black dashed line) and for a methanol-water $\mathrm{x}=$ 0.54 mole fraction mixture (right) at $298 \mathrm{~K}$ (black solid line), mixture at $260 \mathrm{~K}$ (black dashed line) and mixture at $190 \mathrm{~K}$ (black dotted line) In both RDF's the distribution is also shown for pure water (red line) for comparison. . . . . . . . . . . . . . . . . 138

6.13 Partial radial distribution function for OwOw correlations in a methanolwater $\mathrm{x}=0.27$ mole fraction mixture (left) at $293 \mathrm{~K}$ (black solid line) and mixture at $238 \mathrm{~K}$ (black dashed line) and for a methanol-water $\mathrm{x}=$ 0.54 mole fraction mixture (right) at $298 \mathrm{~K}$ (black solid line), mixture at $260 \mathrm{~K}$ (black dashed line) and mixture at $190 \mathrm{~K}$ (black dotted line) In both RDF's the distribution is also shown for pure water (red line) for comparison. . . . . . . . . . . . . . . . . 139

6.14 The triplet bond angle distribution for $O_{W}-O_{W}-O_{W}$ is shown on the left for methanol-water $x=0.27$ at $293 \mathrm{~K}$ (solid black line) and $238 \mathrm{~K}$ (dashed black line) and on the right for methanol-water $x=0.54$ at 298K (solid black line), 260K (dashed black line) and 190K (dotted black line). The triplet bond angle distribution for $O_{W}-O_{W}-O_{W}$ for pure water at $298 \mathrm{~K}$ is also shown on both graphs(solid red line). The vertical line illustrates the position of the tetrahedral angle $109.5^{\circ}$. .

6.15 Partial radial distribution function for CC correlations in a methanolwater $\mathrm{x}=0.27$ mole fraction mixture (left) at $298 \mathrm{~K}$ (black solid line) and mixture at $238 \mathrm{~K}$ (black dashed line) and for a methanol-water $\mathrm{x}=$ 0.54 mole fraction mixture (right) at $298 \mathrm{~K}$ (black solid line), mixture at $260 \mathrm{~K}$ (black dashed line) and mixture at $190 \mathrm{~K}$ (black dotted line). Pure methanol at $298 \mathrm{~K}$ is also shown for comparison(red line). . . . . 142 
6.16 Partial radial distribution function for $\mathrm{CO}$ correlations in a methanolwater $\mathrm{x}=0.27$ mole fraction mixture (left) at $298 \mathrm{~K}$ (black solid line) and mixture at $238 \mathrm{~K}$ (black dashed line) and for a methanol-water $\mathrm{x}=0.54$ mole fraction mixture (right) at $298 \mathrm{~K}$ (black solid line), mixture at 260K (black dashed line) and mixture at 190K (black dotted line). Pure methanol at $298 \mathrm{~K}$ is also shown for comparison(red line). . . . . . . . . 144

6.17 Partial radial distribution function for CM correlations in a methanolwater $\mathrm{x}=0.27$ mole fraction mixture (left) at $298 \mathrm{~K}$ (black solid line) and mixture at $238 \mathrm{~K}$ (black dashed line) and for a methanol-water $\mathrm{x}=0.54$ mole fraction mixture (right) at $298 \mathrm{~K}$ (black solid line), mixture at 260K (black dashed line) and mixture at 190K (black dotted line). Pure methanol at $298 \mathrm{~K}$ is also shown for comparison(red line). . . . . . . .

6.18 Partial radial distribution function for $\mathrm{COW}$ correlations in a methanolwater $\mathrm{x}=0.27$ mole fraction mixture (left) at $293 \mathrm{~K}$ (black solid line) and mixture at $238 \mathrm{~K}$ (black dashed line) and for a methanol-water $\mathrm{x}=$ 0.54 mole fraction mixture (right) at $298 \mathrm{~K}$ (black solid line), mixture at $260 \mathrm{~K}$ (black dashed line) and mixture at $190 \mathrm{~K}$ (black dotted line).

7.1 Composite partial structure factors fits (red lines) obtained by the EPSR computer simulation procedure compared to the original data (black circles) for methanol-water mixture $\mathrm{x}=0.50$ mole fraction at $200 \mathrm{~K}$ and ambient pressure (upper) and $\mathrm{x}=0.50$ mole fraction at $200 \mathrm{~K}$ and 2 kbar (lower). . . . . . . . . . . . . . . . . . . 157 
7.2 Cluster distribution plots for methanol-water $\mathrm{x}=0.50$ mole fraction mixture. Graph shows mixture at $200 \mathrm{~K}$ and ambient pressure (black line) and mixture at $200 \mathrm{~K}$ and $2 \mathrm{kbar}$ (red line) for methanol $\mathrm{CC}$ clusters (left) and water clusters (right) as defined in the text. The dashed lines show the predicted cluster size distribution at the percolation threshold[92]. . . . . . . . . . . . . . 158

7.3 Partial radial distribution function for $\mathrm{CC}$ correlations in a methanolwater $\mathrm{x}=0.50$ mole fraction mixture. Graph shows mixture at $200 \mathrm{~K}$ and ambient pressure (black solid line), mixture at $200 \mathrm{~K}$ and $2 \mathrm{kbar}$ (black dashed line) and pure methanol at $298 \mathrm{~K}$ (red line). . . . . . . . 161

7.4 Partial radial distribution function for OwOw correlations in a methanolwater $\mathrm{x}=0.50$ mole fraction mixture. Graph shows mixture at $200 \mathrm{~K}$ and ambient pressure (black solid line), mixture at $200 \mathrm{~K}$ and 2 kbar (black dashed line) and pure methanol at $298 \mathrm{~K}$ (red line). . . . . . . . 162

7.5 Partial radial distribution functions for Low Density Amorphous (LDA) Ice. Top distribution is $\mathrm{OO}$ correlations, middle distribution shows $\mathrm{OH}$ correlations and lower distribution shows $\mathrm{HH}$ correlations, where $\mathrm{O}$ represents water oxygen atoms and $H$ represents water hydrogen atoms. 163

7.6 Partial radial distribution function for COw correlations in a methanolwater $\mathrm{x}=0.50$ mole fraction mixture. Graph shows mixture at $200 \mathrm{~K}$ and ambient pressure (black solid line) and mixture at $200 \mathrm{~K}$ and 2 kbar (black dashed line). . . . . . . . . . . . . . . . . . . . . . . . . 164

7.7 The triplet bond angle distribution for $O_{W} O_{W} O_{W}$ for pure water at $298 \mathrm{~K}$ (solid red line), methanol-water $x=0.50$ at $200 \mathrm{~K}$ and ambient pressure (solid black line) and $200 \mathrm{~K}$ and $2 \mathrm{kbar}$ (dashed black line). . 164 
7.8 Snapshot from molecular dynamics simulations of an aqueous solution of 10 hydrophobic solutes (methane) in 508 waters at 1 atm (top panel) and 8000 atm (lower panel)[113]. . . . . . . . . . . . . . . . 167

8.1 Schematic of ideal mixing of two liquids A and B to form a homogeneous mixture . . . . . . . . . . . . . . . . 170

8.2 Experimentally measured negative excess entropies, $-\frac{\Delta S}{k N}$ for methanolwater solutions at a range of methanol mole fractions[71] . . . . . . 172

8.3 Schematic illustrating the case of complete demixing of methanol (shown in red) and water (shown in blue) . . . . . . . . . . . . . 173

8.4 Schematic of non-ideal mixing of two liquids $\mathrm{A}$ and $\mathrm{B}$ to form an inhomogeneous mixture . . . . . . . . . . . . . . . . . . . . . 174

8.5 Schematic illustrating a methanol (shown in red) and water (shown in blue) mixture which includes an interfacial region of methanol and water (blue and red hatched region) _. . . . . . . . . . . . 174

8.6 Excess entropy for a range of different methanol water mixtures from experimentally measured values (black line with diamonds), from model with complete demixing (red line with squares), from model using $C O_{W}=3.30 \AA$ definition of clusters (green line with circles), for low temperature measurements (orange traingles) and low temperature and high pressure (blue cross) . . . . . . . . . . . . . . . . . . 179 


\section{List of Tables}

2.1 Intermolecular forces in various types of liquids[24] . . . . . . . . 14

2.2 Relative permittivities, $\epsilon_{r}$, of some non-polar (upper two) and polar (lower two)liquids at $298 \mathrm{~K}$, and dipole moments, $\mu$ of their gaseous molecules. The unit of dipole moment is $D$, the Debye unit, where $1 D$ $=3.338 \times 10^{-30}$ coulomb meters. . . . . . . . . . . 18

2.3 Favourable and Unfavourable Enthalpy Change $\triangle H$ and Entropy Change

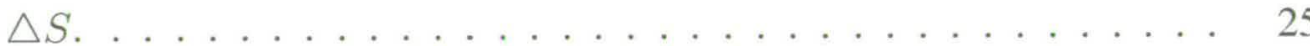

2.4 Classification of solutes according to their Thermodynamic Excess Functions ............................. 26

3.1 Fluorescence emission maximum in relation to concentration for methanolwater solutions from experimental analysis and for ethanol-water solutions from literature[45]. . . . . . . . . . . . . . . . 54

3.2 The fluorescence emission maximum $\lambda_{e m}^{\max }$ for a number of different alcohols and for water, along with their polarity and quantum yield[44] 55

3.3 Fluorescence Decay Parameters of 1,8-anilinonaphthalene sulfonate ANS in Water $\left(\lambda_{e m}=520 \mathrm{~nm}\right)$, Methanol $\left(\lambda_{e m}=490 \mathrm{~nm}\right)$, or MethanolWater $x=0.5,\left(\lambda_{e m}=510 n m\right) \ldots \ldots \ldots$ 
3.4 Global Analysis of a Two Component Mixture of 1,8-anilinonaphthalene sulfonate(ANS) in $x=0.5$ and $x=1$ Methanol-Water solution at $200 \mathrm{~K}$ Measured at 5 Emission Wavelengths, where $\alpha_{1}$ and $\alpha_{2}$ are pre-exponential factors and $f_{1}$ and $f_{2}$ are fractional intensities.

4.1 Scattering lengths and coherent $\left(d \sigma_{c o h}\right)$ and incoherent $\left(d \sigma_{i n c}\right)$ scattering cross-sections for hydrogen, deuterium, carbon and oxygen[81] . .

5.1 Parameters used in Empirical Potential Structural Refinement . . . . . 107

6.1 Parameters of the methanol-water mixtures used in the Empirical Potential Structural Refinement. . . . . . . . . . . . . . . . . . . . .

6.2 Triplet bond angle distributions for $O_{W} O_{W} O_{W}$ for pure water, methanolwater $x=0.27$ and $x=0.54$ at a number of different temperatures after 10000 iterations . . . . . . . . . . . . . . . . 140

7.1 Parameters of the methanol-water mixtures used in the Empirical Potential Structural Refinement. . . . . . . . . . . . . . . . . . . . 156

8.1 Calculation of the negative excess entropy of methanol-water solutions on the basis of a simple model that assumes all the molecules of each component occur in completely segregated clusters of the same density as the pure liquid counterparts. The experimentally observed are also shown for reference[71] . . . . . . . . . . . . . . . 173

8.2 Estimated surface fractions and modified excess entropy of methanolwater solutions, after correcting for the fraction of methanol $\left(f_{m}\right)$ and water $\left(f_{w}\right)$ molecules that occur in the interfacial regions of the respective clusters. 
8.3 Estimated surface fractions and modified excess entropy of methanolwater solutions, after correcting for the fraction of methanol $\left(f_{m}\right)$ and water $\left(f_{w}\right)$ molecules that occur in the interfacial regions of the respective clusters. . . . . . . . . . . . . . . . . . . 183

8.4 Estimated surface fractions and modified excess entropy of methanolwater solutions, after correcting for the fraction of methanol $\left(f_{m}\right)$ and water $\left(f_{w}\right)$ molecules that occur in the interfacial regions of the respective clusters. . . . . . . . . . . . . . . . .

8.5 Estimated surface fractions and modified excess entropy of methanolwater solutions, after correcting for the fraction of methanol $\left(f_{m}\right)$ and water $\left(f_{w}\right)$ molecules that occur in the interfacial regions of the respective clusters. . . . . . . . . . . . . . . . . . 18

B.1 Intermolecular weights for $\mathrm{x}=0.27$ mole fraction methanol water mixture where substitutions are made on the methyl hydrogens. The atom labels $\mathrm{M}, \mathrm{C}, \mathrm{O}$ and $\mathrm{H}$ refer to methanol methyl hydrogen, carbon, oxygen and hydroxyl hydrogen respectively. The atom labels $O_{W}$ and $H_{W}$ refer to water oxygen and hydrogen respectively. In the weights notation $\mathrm{H}$ corresponds to the labelled hydrogen and $\mathrm{X}$ corresponds to the remaining unlabelled atoms. . . . . . . . . . . . . . .

B.2 Intermolecular weights for $\mathrm{x}=0.27$ mole fraction methanol water mixture where substitutions are made on the methanol hydroxyl hydrogens and water hydroxyl hydrogens. The atom labels $\mathrm{M}, \mathrm{C}, \mathrm{O}$ and $\mathrm{H}$ refer to methanol methyl hydrogen, carbon, oxygen and hydroxyl hydrogen respectively. The atom labels $O_{W}$ and $H_{W}$ refer to water oxygen and hydrogen respectively. In the weights notation $\mathrm{H}$ corresponds to the labelled hydrogen and X corresponds to the remaining unlabelled atoms. 195 
B.3 Intermolecular weights for $\mathrm{x}=0.27$ mole fraction methanol water mixture where substitutions are made on all the hydrogens (methyl hydrogens, methanol hydroxyl hydrogens and water hydroxyl hydrogens). The atom labels $\mathrm{M}, \mathrm{C}, \mathrm{O}$ and $\mathrm{H}$ refer to methanol methyl hydrogen, carbon, oxygen and hydroxyl hydrogen respectively. The atom labels $O_{W}$ and $H_{W}$ refer to water oxygen and hydrogen respectively. In the weights notation $\mathrm{H}$ corresponds to the labelled hydrogen and $\mathrm{X}$ corresponds to the remaining unlabelled atoms.

B.4 Intermolecular weights for methanol-water $x=0.54$ mole fraction mixture where substitutions are made on the methanol methyl hydrogens (note. it is only the $\mathrm{M}$ correlation weights which change between different weights files) The atom labels $\mathrm{M}, \mathrm{C}, \mathrm{O}$ and $\mathrm{H}$ refer to methanol methyl hydrogen, carbon, oxygen and hydroxyl hydrogen respectively. The atom labels $O_{W}$ and $H_{W}$ indicate water oxygen and water hydrogen. . . . . . . . . . . . . . . . . . . . . . . 197

B.5 Intermolecular weights for methanol-water $x=0.54$ mole fraction mixture where substitutions are made on methanol hydroxyl hydrogens and water hydroxyl hydrogens (note. it is only the $\mathrm{H}$ correlation weights which change between different weights files) The atom labels $\mathrm{M}, \mathrm{C}, \mathrm{O}$ and $\mathrm{H}$ refer to methanol methyl hydrogen, carbon, oxygen and hydroxyl hydrogen respectively. The atom labels $O_{W}$ and $H_{W}$ indicate water oxygen and water hydrogen. . . . . . . . . . . . . . . 198 
B.6 Intermolecular weights for methanol-water $x=0.54$ mole fraction mixture where substitutions are made on all hydrogens (note. it is only the $\mathrm{M}$ and $\mathrm{H}$ correlation weights which change between different weights files) The atom labels $\mathrm{M}, \mathrm{C}, \mathrm{O}$ and $\mathrm{H}$ refer to methanol methyl hydrogen, carbon, oxygen and hydroxyl hydrogen respectively. The atom labels $O_{W}$ and $H_{W}$ indicate water oxygen and water hydrogen.

B.7 Intermolecular weights for the 50:50 methanol-water mixture where substitutions are made on the methyl hydrogens. The atom labels $\mathrm{M}$, $\mathrm{C}, \mathrm{O}$ and $\mathrm{H}$ refer to methanol methyl hydrogen, carbon, oxygen and hydroxyl hydrogen respectively. The atom labels $O_{W}$ and $H_{W}$ indicate water oxygen and water hydrogen. . . . . . . . . . . . . 200

B.8 Intermolecular weights for the 50:50 methanol-water mixture where substitutions are made on the methanol hydroxyl hydrogen and the water hydroxyl hydrogens. The atom labels $\mathrm{M}, \mathrm{C}, \mathrm{O}$ and $\mathrm{H}$ refer to methanol methyl hydrogen, carbon, oxygen and hydroxyl hydrogen respectively. The atom labels $O_{W}$ and $H_{W}$ indicate water oxygen and water hydrogen. . . . . . . . . . . . . . . . . . 20

C.1 Coordination numbers for pure methanol obtained from integration of the peaks observed in the partial pair distribution functions at $298 \mathrm{~K}$ obtained from the EPSR analysis[106] . . . . . . . . . . . . . . . . . 204

C.2 Coordination numbers for pure water obtained from the integration of the peaks observed in the partial pair distributions functions at $298 \mathrm{~K}$ obtained from the EPSR analysis[126] . . . . . . . . . . . . . . . 204

C.3 Coordination numbers for methanol -water solution mole fraction $\mathrm{x}=$

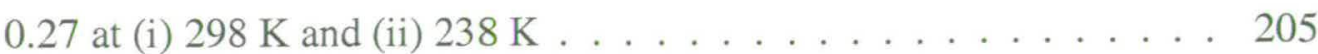


C.4 Coordination numbers for methanol -water solution mole fraction $\mathrm{x}=$ 0.54 at (i) $298 \mathrm{~K}$ and (ii) $260 \mathrm{~K}$ and (iii) $190 \mathrm{~K} \ldots \ldots . . . . . . .206$

C.5 Coordination numbers for methanol -water solution mole fraction $\mathrm{x}=$ 0.54 at (i)298 K and (ii) $260 \mathrm{~K}$ and (iii)190 K . . . . . . . . . . . . 207

C.6 Coordination numbers for methanol -water solution mole fraction $\mathrm{x}=$ 0.50 at (i) $200 \mathrm{~K}$ and ambient pressure and (ii) $200 \mathrm{~K}$ and $2 \mathrm{kbar}$. . 208 


\section{Chapter 1}

\section{Introduction}

\subsection{Crucial Role of Water in Biology}

Water is an important substance and has been the inspiration of poets, painters, composers, philosophers and scientists over the years. Water has the wonderful ability to dissolve an extensive range of compounds making it a superbly fit environment to support life. As a solvent it is the vehicle in which ions or other compounds are transported through the body and is responsible for their ready access to cells and tissues and ready elimination from the body. It has become clear that aqueous environment is far from being just an inert, 'blank canvas' on which complicated biochemical events take place. Water is being recognised as an active participant in biological processes in the sense that it responds structurally and dynamically to the presence of other molecules in subtle and non-intuitive ways. Without water, biomolecules would be left stranded or immobile, like 'beached whales' [1]. They might also no longer truly be biomolecules, unravelling or seizing up and losing their biological function in the process. In living cells, the fluid inside is called cytoplasm and is made up of mostly water (Figure 1.1). However, it also contains proteins, DNA, sugars, salts and fatty acids.

Attempts to understand the role of water in the cell, face a circular dilemma. Firstly, 


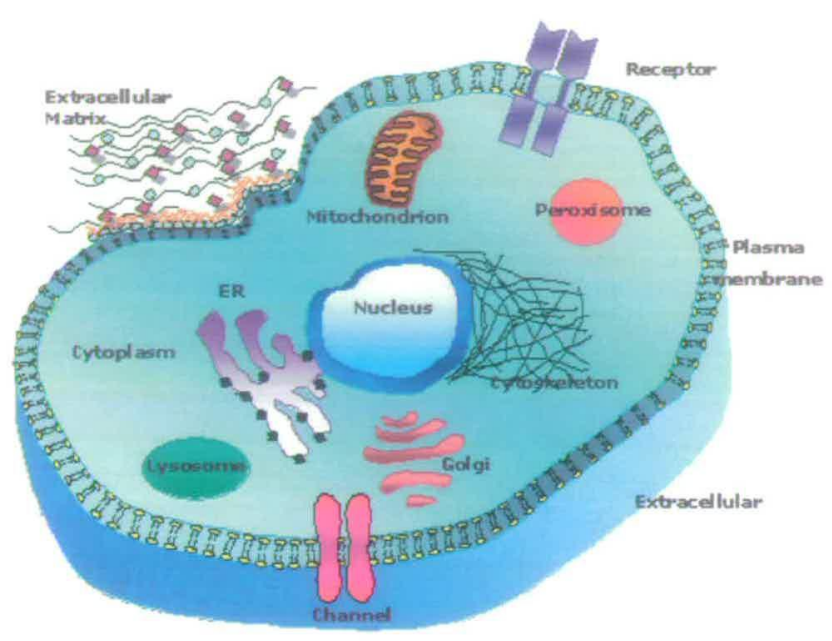

Figure 1.1: The Living Cell

it is necessary to ask how the solvent properties of water influence the character and behaviour of the solutes from simple metal ions to large, complicated proteins. Secondly, there are critical and still mysterious questions about how the presence of these solutes might modify the very ability of water to act as a solvent. There are a number of crucial areas where important questions still remain and research is very active in trying to find some answers.

Proteins are complex, organic biomolecules containing many amino acid groups linked together by covalent peptide bonds. Amino acids are organic acids containing at least one carboxyl group $\mathrm{COOH}$ and at least one amino group $\mathrm{NH}_{2}$. These amino acids are of great importance because they combine together to form proteins. Amino acids form peptides by the reaction of adjacent amino $\mathrm{NH}_{2}$ and carboxyl $\mathrm{COOH}$ groups. Proteins are then polypeptide chains consisting of hundreds of amino acids. Living cells use 21 amino acids and since proteins have hundreds to thousands of amino acids in each molecule, the number of possible proteins is very large. The order of amino acids in proteins is controlled by genes in the cells DNA. The most important proteins are enzymes, which determine all the chemical reactions in the cell and antibodies which combat infection. Structural proteins include keratin and collagen. Gas transport 


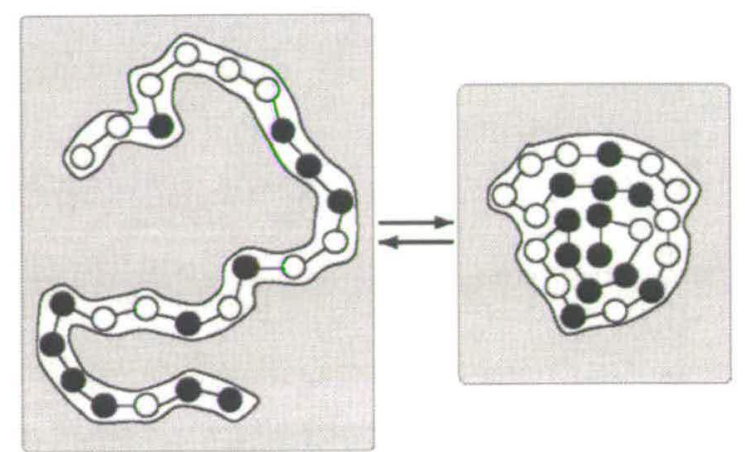

Figure 1.2: Illustration of a cartoon protein containing hydrophobic (black circles) and hydrophilic (white circles) regions. In aqueous solution the hydrophobic regions are buried in the core, shielded by the hydrophilic regions from surrounding water.

proteins include hemoglobin. Muscle proteins include actin and myosin.

Proteins are made up of a large number of hydrophobic, 'water-fearing', and hydrophilic, 'water-loving' regions. This is because their amino acid building blocks have varying degrees of solubility. When surrounded by water, proteins 'gather together' the hydrophobic parts and 'cover them' with the hydrophilic parts. To do this, the protein chain of amino acids folds up into a well defined shape with the hydrophobic regions packed into a buried core shielded by the hydrophilic regions which are at the surface exposed to the surrounding water (Figure 1.2).

A protein's behaviour or function is determined largely by its 3-dimensional shape. A protein's native state is the folded state. For some proteins, if they lose their 3dimensional shape and become strung out in a floppy, loose chain, the protein is no longer able to do its job. The process of altering the native/low free energy conformation of a protein is called denaturation. Denatured proteins do not retain their original properties or biological functions. The antibodies of the immune system are proteins that have binding pockets sculpted simply for seizing onto foreign particles in the body. Thus, their specialised shape is a crucial part of performing their purpose or function. The coiled shape of structural proteins in the bodies tissues, for example collagen in bone, determines the strength, flexibility and elasticity of fibrous tissue. 


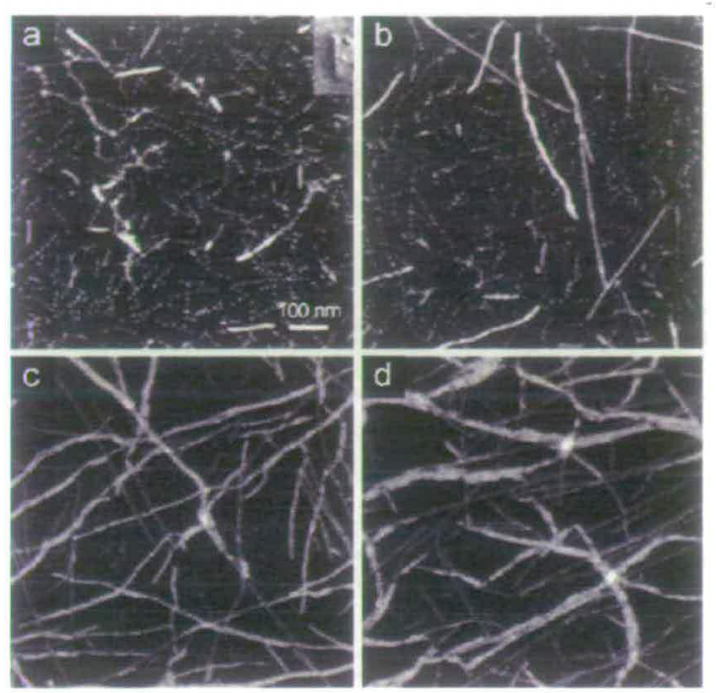

Figure 1.3: Intermediate structures in the self assembly of the small peptide KFE8 in aqueous solution. The images are Atomic Force Microscopy scans at different times after preparation of solution (a) after 8 minutes, (b) 35 minutes, (c) 2 hours and (d) 30 hours[5].

The important point is that the hydrophobic effect is considered to be the driving force for the folding of globular proteins. It results in the burial of the hydrophobic residues in the core of the protein. It is clear that an understanding of protein folding cannot neglect the envelope of water surrounding the protein.

Almost all biological macromolecules (proteins, enzymes and DNA) are inactive in the absence of water. The hydration shell formed by water molecules in the close vicinity to a protein or enzyme is particularly important for the stability of the structure and the function or recognition at a specific site. The role of hydration in enzyme catalysis is well known and has been reviewed in a number of publications[2], [3], [4]. In one of these recent studies[2] it was shown that the dehydration of a protein, which makes it more rigid and increases its denaturation temperature, is correlated with the loss of its physiological function. An understanding of the dynamics of water molecules at the surface of the protein, with molecular structure and dynamical information is important for elucidating a molecular picture of these complex and important systems.

Type-I peptides (containing hydrophobic and hydrophilic surfaces) form beta sheets 
in aqueous solution. Like Lego bricks that have pegs and holes and can only be assembled into particular structures, these peptides can do so at the molecular level. Figure 1.3 shows the intermediate structures in the self assembly of the small peptide KFE8 in aqueous solution. The images are Atomic Force Microscopy scans at different times after preparation of solution (a) after 8 minutes, (b) 35 minutes, (c) 2 hours and (d) 30 hours. The tendency of these so-called 'molecular Lego' molecules to selfassemble into hydrogel scaffolds is being harnessed for potential tissue engineering applications[5]. However, detailed molecular level descriptions of hydrophobicity are the subject of conflicting models and fundamental issues remain controversial. One basic problem existing at both small and large length-scale regimes is the molecular structure of the water-amphiphile interface[6], [7]. A satisfactory resolution to this issue in the important biomolecular context is made worse by the structural complexity of the relevant molecules. Recent X-ray SAXS results on the proteins lysozyme and protein $\mathrm{R} 1$ of E.coli ribonucleotide reductise showed enhanced scattering at the protein-solvent interface relative to that from free water, implying higher density in the hydration shell[8]. This work is in agreement with some molecular dynamics simulations by Levitt et al[9].

However, this work contradicts the traditional Frank and Evans 'iceberg' picture[10]. In the iceberg model the hydrophobic hydration shell is more ordered (ice-like) than in bulk water and consequently of lower density. Also, if there were a hydration shell around proteins of mean density different (higher or lower) from that of the bulk solvent then this would lead to a solvent-dependent, partial specific volume for the protein, which is not observed.

A compromise hypothesis is that because of the heterogeneous nature of macromolecular surfaces, the hydration shell could have, locally, a higher or lower density than the bulk. An example would be with carbohydrates which may be considered as having two hydrophobic surfaces with a hydrogen bonding edge. The idea is that the packing around hydrophobic groups would lead to lower density whereas electrostriction 
around charged groups would lead to higher density. Since half of the protein surface is polar and half is non-polar, the effect of density variations would cancel out yielding a mean shell density similar to that of the bulk. Surface topography also appears to play an important role for biomolecules having a variety of concave, convex and flat surface regions[11].

\subsection{A Model System}

A promising route to the development, testing and refinement of detailed molecular models of the hydrophobic interaction, hydration and the physics of aqueous macromolecules includes the use of small molecule systems as 'prototypes'.

The criteria that a useful model amphiphilic systems must satisfy is that

1. They must show extreme structural simplicity

2. Their aqueous solutions must exhibit thermodynamic non-ideality

3. The model amphiphiles must show some tendency to self-organise in water

4. The 'organisation' must be consistent with hydrophobic-like structures whereby the apolar groups are in contact and sequestered from the aqueous environment.

Methanol is one of the simplest amphiphile like molecules. It has a methyl headgroup which is non-polar and an $\mathrm{OH}$ group which is polar. The non-polar methyl group is hydrophobic (water-fearing), and the polar hydroxyl group is hydrophilic (waterloving).

Water-alcohol mixtures can be used to develop a molecular level understanding of the hydrophobic interaction. They are simple amphiphiles and provide a means to study the hydrophobic interaction of a simple system. The solubility of an alcohol in water depends on the length of the hydrophobic alkyl headgroup. The higher 
and therefore longer alcohols, such as n-butanol and n-octanol ${ }^{1}$, show significant immiscibility when mixed with water. In the past it was widely accepted that since simple alcohols, such as methanol and ethanol, are completely miscible in water, they must be homogeneously mixed. However, recent experimental and computational studies on aqueous methanol have revealed unexpectedly complex behaviour at medium length-scales leading to a substantially revised view of miscibility in this prototype aqueous amphiphile[12, 13, 14]. In particular, molecular-level segregation has been observed with structural details consistent with those expected for a hydrophobically-driven system[12, 13]. The thermodynamic factors that govern hydrophobically driven systems involve the competing forces of enthalpy and entropy. The temperature dependence of these two terms are not the same and it is expected that the hydrophobic interaction will show an appreciable dependence on temperature. Temperature induced effects related to hydrophobicty are particularly dramatic in aqueous solutions of biological macromolecules where processes such as thermal denaturation of proteins are familiar[15]. Recent reports (from simulation[16] and dielectric spectroscopy[17]) on low-temperature dynamical properties of so called hydration water in aqueous protein solutions are offering new probes of hydration processes. These studies are also contributing to the ongoing debate over the peculiar properties of supercooled water[18, 19] where continued controversy[20] surrounds suspected divergences of certain thermodynamic functions at inaccessibly low temperatures in pure liquid water[19, 21].

\subsection{Exploring Aqueous Alcohol Solutions}

High resolution picosecond fluorescence spectroscopy was used to study methanolwater solutions over a range of concentrations. This powerful, high through-put optical

\footnotetext{
${ }^{1}$ where the $\mathrm{n}$ implies that it is the normal configuration of the molecule, with the $\mathrm{OH}$ group at the end of the chain
} 
technique allowed exploration over a vast range of state points and conditions. In order to answer fundamental physical questions about the molecular and bulk properties of a system work was carried out for the first time on the fluorescence spectrometer arrangement in the low temperature and supercooled regime revealing very interesting results. Neutron diffraction with hydrogen/deuterium isotope substitution combined with Empirical Potential Structural Refinement (EPSR) has been used to investigate methanol-water systems across a wide range of concentrations and state points.

\subsection{Thesis Layout}

- Chapter 2: This chapter will present the background theory for investigating aqueous systems. The chapters begins with an introduction to water and the idea of structure in liquids. Aqueous amphiphiles are then introduced as model aqueous systems and the different interactions they experience are discussed in detail. The chapter then gives a review of the current understanding of aqueous amphiphiles from neutron diffraction work, computer simulations and other experimental techniques.

- Chapter 3: This chapter presents results from fluorescence spectroscopy experiments on probing the structure and dynamics of a methanol-water mixture. It begins with an introduction to the experimental technique of fluorescence spectroscopy with particular emphasis on the importance of the fluorescence probe.

- Chapter 4: The experimental technique of neutron diffraction will be described. The chapter begins with a description of the diffraction experiment and introduces the theory behind diffraction. The important technique of isotope substitution is explained and its relevance in real liquid systems. The data correction procedures which must be performed on the data are summarised. Finally, the 
computational modelling technique, the Empirical Potential Structural Refinement is presented and the key features explained.

- Chapter 5: The neutron diffraction results from a study of a range of different concentrations of methanol-water mixtures are presented.

- Chapter 6: The neutron diffraction results from a study of the effects of cooling on two methanol-water solutions are presented.

- Chapter 7: Neutron Diffraction results from a study on the effects of compression on methanol-water mixtures are presented

- Chapter 8: A simple model will be introduced which attempts to explain the negative excess entropy observed in alcohol-water solutions.

- Chapter 9: The thesis concludes with a summary of the main findings and thoughts on avenues for future work in this field 


\section{Chapter 2}

\section{Background Theory}

The aqueous environment can provide a wealth of information about the behaviour of water and the important role it has to play. This chapters begins with an introduction to water and its mysterious properties and the idea of structure in liquids. Aqueous amphiphiles are then introduced as model aqueous systems and the different interactions they experience are discussed. The chapter then gives a review of the current understanding of aqueous amphiphiles.

\subsection{Water- A Very Important Substance}

Water covers $70 \%$ of the earth's surface and is the most widely used solvent on the planet. It is essential to life and makes up $60-70 \%$ of the human body. There have been a huge number of studies on water not only because it is the most important liquid on earth, but also because it has so many interesting and anomalous properties. For example, it takes a relatively large amount of heat to raise the temperature of water by one degree. This enables the world's oceans to store enormous amounts of heat, producing a moderating effect on the world's climate. Liquid water expands when cooled below $4^{\circ} \mathrm{C}$, unlike most liquids which expand only when heated. Ice is 


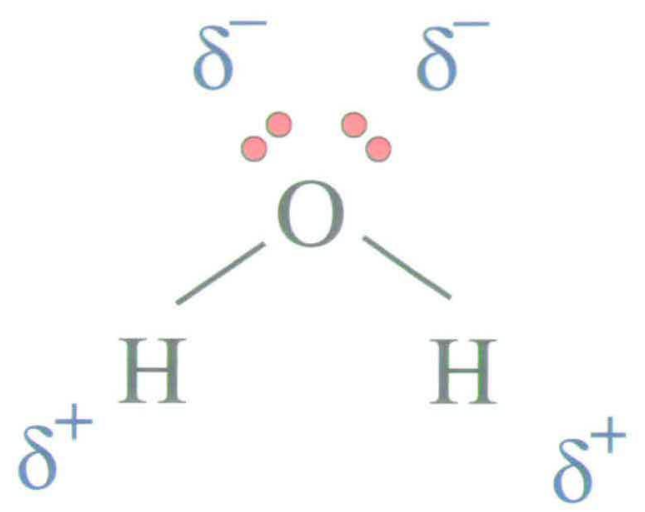

Figure 2.1: Illustration of a water molecule showing the oxygen atom, $O$, with 2 lone pairs of electrons (shown as small red circles) and consequently a partial -ve charge and the two hydrogen atoms $H$ with a partial +ve charge

less dense than liquid water, as can be seen in icebergs floating above the water and when the top layers of ponds freeze over. For a liquid of such low molecular weight, water has unexpectedly high melting and boiling points and latent heat of vaporisation. There are many other substances of low molecular weight and high melting and boiling points, but these are invariably ionic crystals or metals whose atoms are held together by strong colombic or metallic bonds. These properties of water point to the existence of an intermolecular interaction that is stronger than expected for ordinary liquids.

Water has the familiar chemical formula $\mathrm{H}_{2} \mathrm{O}$ and is angular in structure with $104.5^{\circ}$ between hydrogens. If we consider the two lone pairs of electrons, the molecule is tetrahedral[22]. There is an uneven charge distribution within the $\mathrm{O}-\mathrm{H}$ bonds such that there is a +ve partial charge localised around the hydrogens and -ve partial charge associated with the oxygen (Figure 2.1).

The -ve partial charge is twice the magnitude of each hydrogen partial charge. Water is capable of forming a very special type of bond called a hydrogen bond. A water molecule can form up to 4 hydrogen bonds: each hydrogen can serve as a donor, and the oxygen (with 2 sets of unpaired electrons) can participate as a hydrogen bond acceptor in 2 interactions. As a result of this ability, and the tetrahedral arrangement of the donor/acceptor groups, water can potentially form an extensive hydrogen bonded 


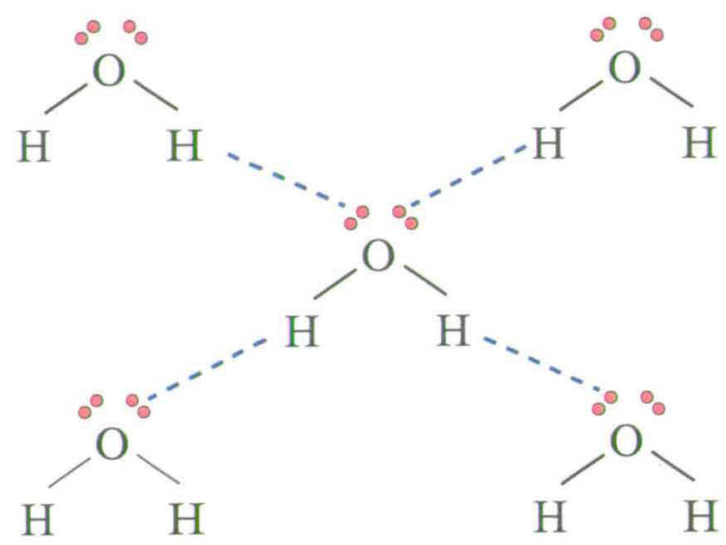

Figure 2.2: Illustration of hydrogen bonded network with each water molecule participating in 4 hydrogen bonds. Each hydrogen acting as a donor for a hydrogen bond and oxygen acting as an acceptor for hydrogen bonds. Hydrogen bonds are shown as blue dashed lines, while covalent bonds are shown as black lines.

network[22] (Figure 2.2).

Hydrogen bonds are not unique to water; they exist to varying degrees between electronegative atoms such as; oxygen, nitrogen and chlorine and hydrogen atoms covalently bound to similar electronegative atoms. These bonds are special in that they only involve hydrogen atoms, which by virtue of their tendency to become positively polarised and their uniquely small size, can interact strongly with nearby electronegative atoms resulting in a hydrogen bond between two electronegative atoms. Hydrogen bonds occur intermolecularly as well as intramolecularly and can happily exist in a non-polar environment. They are consequently very important in macromolecular and biological assemblies such as proteins[23], linking different segments together inside the molecules, and in nuclei acids where they are responsible for the stability of the DNA molecule holding the 2 helical strands together. Therefore, hydrogen bonds have a very important role to play in biological systems.

All molecules experience intermolecular attractions, although in some cases these are very weak[24]. Van der Waals dispersion forces, also known as London forces are another type of intermolecular attraction. These arise due to temporary dipoles which give rise to intermolecular attractions. Dispersion forces between molecules are much 


\begin{tabular}{|c|c|c|}
\hline Intermolecular Force & $\begin{array}{c}\text { Approx Strength } \\
\mathrm{kJ} \mathrm{mol}^{-1}\end{array}$ & $\begin{array}{c}\text { Energy } \\
\mathrm{kT} / \mathrm{molecule}\end{array}$ \\
\hline Covalent bonds & $100-1000$ & $40-400$ \\
Ionic interactions & 15 & 6 \\
Van der Waals (dispersion) & $1-5$ & $0.4-2$ \\
Van der Waals dipole-dipole & $1-10$ & $0.4-4$ \\
Hydrogen Bonds & $10-50$ & $4-20$ \\
\hline
\end{tabular}

Table 2.1: Intermolecular forces in various types of liquids[24]

weaker than the covalent bond within molecules and the size of the attraction varies considerably with the size of the molecule and its shape.

Dipole-dipole interactions are another form of Van der Waals attractions. A molecule like $\mathrm{HCl}$ has a permanent dipole because $\mathrm{Cl}$ is more electronegative than $\mathrm{H}$. These permanent, in-built dipoles will cause the molecules to attract each other rather more than they otherwise would if they had to rely only on dispersion forces. Therefore, depending on the molecule, different intermolecular forces will play an important role. A classification of liquids can be thought of in terms of the intermolecular forces involved (Table 2.1). These interactions will determine the P-V-T phase diagram of the liquid and is therefore characteristic for a given substance. Hydrogen bonds have about a tenth of the strength of an average covalent bond, and are being constantly broken and reformed in liquid water. If the covalent bond between the oxygen and hydrogen is likened to a stable marriage (bond strength $100-1000 \mathrm{~kJ} \mathrm{~mol}^{-1}$ ), the hydrogen bond has 'just good friends' status $\left(10-50 \mathrm{~kJ} \mathrm{~mol}^{-1}\right)$. On the same scale, van der Waals attractions represent mere 'passing acquaintances' $\left(0-10 \mathrm{~kJ} \mathrm{~mol}^{-1}\right)[24]$. 


\subsection{Structure in Liquids}

The structure of a solid is generally defined by the coordinates of atoms or molecules and contains an element of periodicity. A liquid, on the other hand, is characterised by random diffusion or Brownian motion of molecules and is absent of periodic order. This means that the liquid state cannot be described by a set of molecular coordinates like a crystal structure. Liquid structure can still be studied. Investigations carried out using $\mathrm{x}$-ray and neutron diffraction experiments have found that liquids, like crystalline solids, scatter radiation, and that fairly well defined patterns of electron or neutron intensity can be measured.

There is an important distinction between structure in solids and in liquids. What in the solid state is simply measured as an electron density, in the liquid state becomes a probability electron or neutron density. This probability density indicates the level of probability that certain positions are occupied by molecules at certain times.

This probability distribution can be visualised in the radial distribution function $g(r)$ from which much information can be gained about the structure of a liquid. Consider a system of particles in a scattering medium and 1 of the particles, $\alpha$, is chosen as the origin. The radial distribution function, $g_{\alpha \beta}(r)$, is obtained by determining the number of particles, $\beta$, whose centres lie within a distance $\mathrm{dr}$ of a circle of radius $\mathrm{r}$ centred around the origin, $\alpha$, averaged over all atoms in the configuration. Then, $r^{2} g_{\alpha \beta}(r) d r$ is proportional to the probability of finding an atom in a shell $r$ away of thickness $d r$. The general shape for the $g_{\alpha \beta}(r)$ of a liquid is shown (Figure 2.3). If $g_{\alpha \beta}(r)$ is large for a given value of $r$, there is a preferred molecular spacing, rather than a completely random arrangement of molecules, in which case $g_{\alpha \beta}(r)=1$. For low $r$ values $g(r)$ is zero, i.e. there is zero probability of finding a molecule at that value of $r$. This is the hard core repulsion which restricts the approaching atom from coming too close and prevents atomic overlap. Molecules are almost incompressible and they strongly repel each other as they approach a distance of separation given by the Van der Waal 


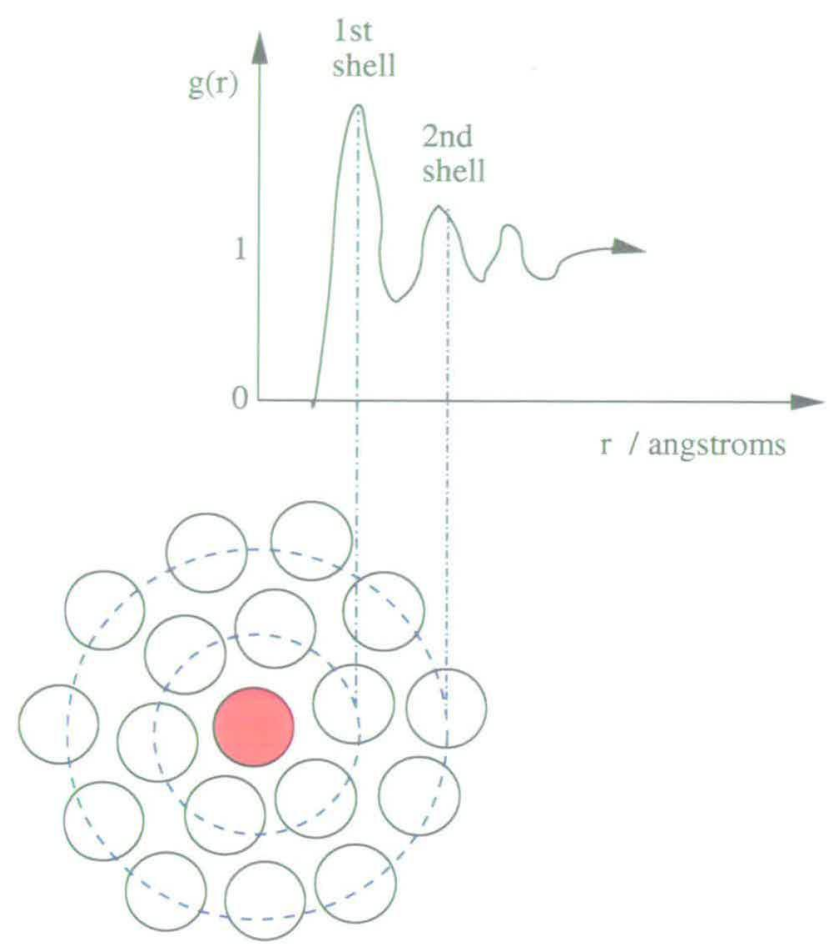

Figure 2.3: Schematic of a typical radial distribution function for a liquid showing the first and second coordination shell (blue lines)

diameter of the molecule. The 1st peak in Figure 2.3 denotes the distance at which the 1st shell of nearest neighbour exists. Correspondingly, the 2 nd peak denotes the distance at which the 2 nd shell of nearest neighbours exists. As the distance from the reference atom increases the oscillations in $\mathrm{g}(\mathrm{r})$ diminish rapidly and the function approaches unity (since $\mathrm{g}(\mathrm{r})$ is normalised to average atomic density of the liquid). Structural correlations in a liquid are short range and extend over a few molecular diameters, beyond which the molecular arrangement becomes uniform and random, with no preferential intermolecular spacings.

Integration under the 1 st peak of a radial distribution functions gives the 1 st neighbour coordination number, i.e the number of atoms in the 1st coordination shell in Figure 2.3. The coordination number can be calculated using 


$$
4 \pi \rho \int_{r=0}^{r=R_{\min }} r^{2} g(r) d r
$$

where $\rho$ is the atomic number density of the particular sample, and $R_{\min }$ is the position of the first minimum in $\mathrm{g}(\mathrm{r})$ after the first peak/coordination shell.

\subsection{The Aqueous Environment}

\subsubsection{Polar and Non-Polar Liquids}

Substances that dissolve readily in water are generally polar. Polar liquids have an uneven charge distribution and therefore have an electric dipole moment. Water and hydrogen fluoride are both polar substances (Table 2.2). Water has a significant negative charge concentrated on its oxygen and a positive charge on the hydrogen. Therefore polar molecules have regions that are positively charged and regions which are negatively charged. Polar liquids interact via dipole-dipole interactions. These interactions are strong, $\sim 40 \mathrm{kJmol}^{-1}$. If the liquid contains hydrogen and an -ve charged atom then the liquids may interact via hydrogen bonds. Water dissolves polar molecules so well because it itself is polar, and so gives to the solute molecules a stabilising attraction between opposite charges. Some biological molecules, such as sugars are considerably polar and are therefore highly soluble in water.

Non-polar molecules have an even charge distribution and an electric dipole moment of zero. Carbon chloride, $\mathrm{CCl}_{4}$, and $\mathrm{C}_{6} \mathrm{H}_{6}$ in Table 2.2 are examples of non-polar liquids. The positive and negative charges are spread evenly throughout the individual molecules. Non-polar liquids interact via Van der Waals dispersion forces, which are weak interactions. 


\begin{tabular}{|c|c|c|}
\hline Molecules & $\epsilon_{r}$ & $\mu / D$ \\
\hline $\mathrm{CCl}_{4}$ & 2.23 & 0 \\
$\mathrm{C}_{6} \mathrm{H}_{6}$ & 2.27 & 0 \\
$\mathrm{H}_{2} \mathrm{O}$ & 78.4 & 1.85 \\
$\mathrm{HF}$ & 175 & 1.82 \\
\hline
\end{tabular}

Table 2.2: Relative permittivities, $\epsilon_{r}$, of some non-polar (upper two) and polar (lower two)liquids at $298 \mathrm{~K}$, and dipole moments, $\mu$ of their gaseous molecules. The unit of dipole moment is $D$, the Debye unit, where $1 D=3.338 \times 10^{-30}$ coulomb meters.

\subsubsection{Amphiphiles}

Many biological molecules are made up of parts that are polar and non-polar. These molecules are known as amphiphiles. In the presence of water, the polar regions are said to be hydrophilic or 'water-loving', while the non-polar regions are said to be hydrophobic or 'water-fearing'. Amphiphilic molecules can spontaneously self-assemble in aqueous solution to form super-structures[23]. This self-assembly is driven by the two extreme responses the hydrophilic and hydrophobic groups have to the presence of water. To illustrate this important effect consider an important example.

Cell membranes are made primarily of tadpole-shaped molecules called phospholipids, which have a polar, hydrophilic head and a non-polar, hydrophobic tail (Figure 2.4). The phospholipid molecules are packed side by side in sheets in the cell walls with their heads and tails aligned. The sheets are arranged back to back in double layers with the tails of the molecules in one sheet meeting those in the other. This molecular arrangement allows the hydrophobic tail regions to be shielded from water by the hydrophilic head regions (Figure 2.4). 


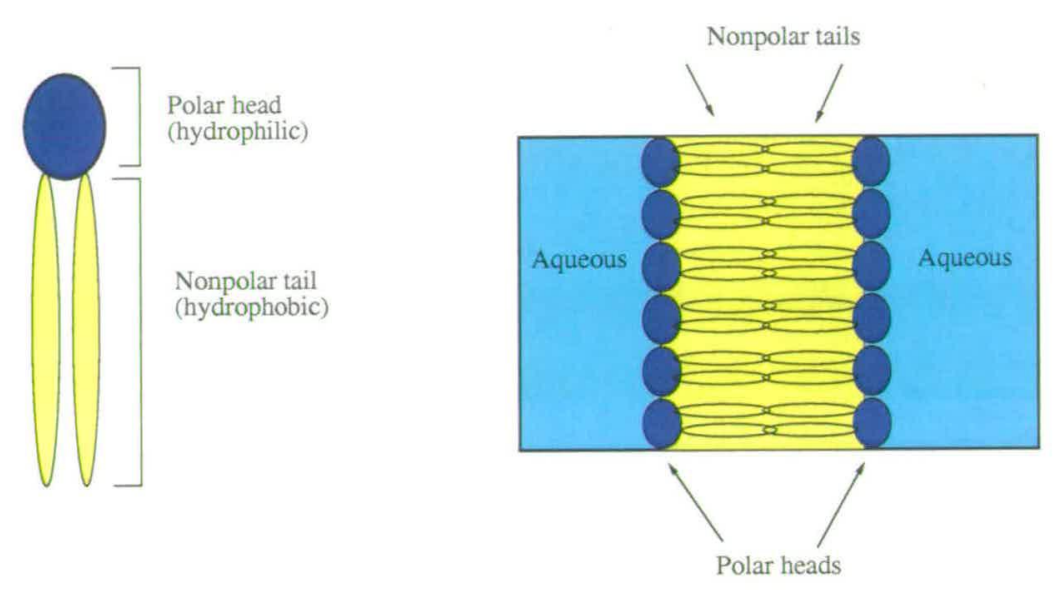

Figure 2.4: Cartoons illustrating a phospholipid with polar hydrophilic head and non-polar hydrophobic tail (left) and the lipid bilayer formed (right)

\subsubsection{The Hydrophobic Interaction}

In bulk water the molecules are joined together into a vast, random network of hydrogen bonds. Consider what happens to this hydrogen bonded network when something is added to water. Adding a solute to water will disturb its hydrogen bonding network. If the solute is capable of forming hydrogen bonds, new hydrogen bonds will be formed between water molecules and solute molecules. However consider what happens if the solute molecule is incapable of forming hydrogen bonds. There has been a great motivation to understand how water responds to the presence of hydrophobic 'invaders'[1].

Methane is a small hydrophobic molecule. Its chemical formula is $\mathrm{CH}_{4}$. It cannot form hydrogen bonds as it does not have an electric dipole to enable it to exploit favourable electrostatic interactions with a polar solvent like water. When methane is added to water the solution warms up. This is an exothermic reaction and energy is released. This is a sign that bonds or interactions are being formed in the solution and the methane molecules are taking part in favourable interactions. Any breaking of bonds as methane forces out a cavity in the water network is more than balanced out by other favourable interactions, which can only be due to Van der Waals forces, between 
methane and surrounding water.

This is all good, however, methane does not dissolve well in water. This is because heat change is only part of the story. Whether or not a process will occur (i.e. whether water dissolves methane) depends on the balance between two different forces, enthalpy (heat change) and entropy. The favourable heat change is counteracted by an unfavourable entropy change. Entropy is a measure of the disorder in a system. When methane is added to water the entropy in the system decreases and there is more order. This means that when methane is introduced into water the overall system is more ordered than the constituent parts. The presence of a hydrophobic molecule like methane amidst the random hydrogen-bonded network of liquid water somehow increases the structure in the water. The preference of water molecules to form hydrogen bonds with each other has a strong influence on their interaction with non-polar molecules that are incapable of forming hydrogen bonds. It would appear that whichever way the water molecules face one or more of the four charges per molecule will have to point toward the non-polar solute molecule. The best configuration would have the least number of tetrahedral charges pointing toward the non-polar species so that the other charges can point toward the water phase and so be able to participate in hydrogen bond attachments as before. If the non-polar solute molecule is not too large, it is possible for water to pack around it without giving up any of their hydrogen bond sites.

When methane is introduced into water some of the waters will organise into a clathrate (pentagon dodecahedron) cage[25] which surrounds the methane (Figure 2.5). The hydrogen bonds are not stronger than in pure water, but the water molecules forming these cages are more ordered than in bulk water. This cage has the characteristic that each water molecule is positioned such that no potential hydrogen bonding donor or acceptor group is positioned toward the centre of the cage. The result is that each water can still completely satisfy its hydrogen bond requirement while the non-polar group resides inside the cage. 


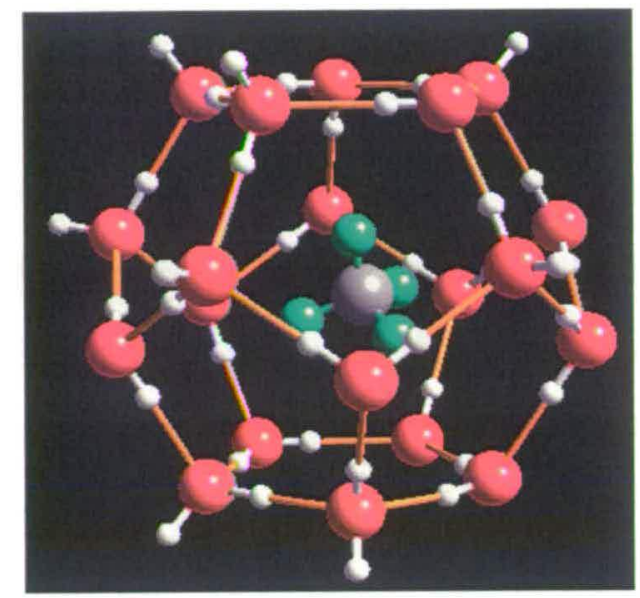

Figure 2.5: Clathrate Cage of Water Surrounding Hydrophobic Methane Molecule[25]

This is known as hydrophobic solvation or hydrophobic hydration. The restructuring or reorientation is entropically very unfavourable, since it disrupts the existing water structure and imposes a new more ordered structure on the surrounding water molecules. It is for this reason that hydrocarbons like methane are so sparingly soluble in water. The immiscibility of substances with water, and the mainly entropic nature of this incompatibility is known as the hydrophobic effect.

In a classic paper Frank and Evans[10] proposed water molecules at the surface of the cavity created by the non-polar solute must be capable of rearranging themselves in order to regenerate the broken hydrogen bonds, even generating stronger hydrogen bonds than those that have been broken.

'When a rare gas atom or a non-polar molecule dissolves in water at room temperature it modifies the water structure in the direction of greater 'crystallinity'-the water, so to speak, builds a microscopic iceberg around it.' [10]

The term iceberg was used to represent 'a microscopic region, either of pure water or surrounding a solute molecule or ion, in which water molecules are tied together in some sort of quasi-solid structure.' [10] However, the term 'ice-berg' is potentially misleading. It implies that the proposed solvent cage is virtually crystalline and composed 

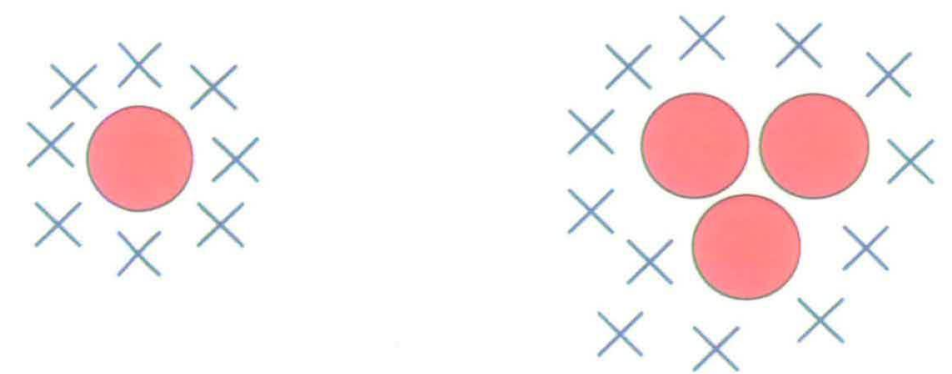

Figure 2.6: Illustration of hydrophobic hydration (left) and hydrophobic interaction (right) where the blue crosses represent water and the red circles represent a hydrophobic species

of ice-like patterns of hydrogen bonds.

In 1959, Walter Kauzmann recognised the true nature of the hydrophobic attraction and clustering must lie within the entropy of hydration. According to the Frank and Evans model[10], hydrophobic molecules in water are surrounded by hydration shells in which water molecules are more highly structured and so have increased order or structure (lower entropy). If two such molecules are brought together so that they are touching, the hydration shells overlap. This means that some of the 'structured' water molecules in these shells can be released. Therefore, the total amount of water needed to hydrate the two particles is less than when they are together. The amount of enhanced water structure, or of entropy reduction, imposed by the hydrophobic solutes is therefore reduced when they are brought together.

This apparent force of attraction is called the hydrophobic interaction and it arises from the preference for maximal disorder. The Kauzmann model only makes sense if the hydration shells of hydrophobic particles really do contain more highly structured water. This has proved difficult to find true as will be discussed in the review of work on aqueous solutions in a later section in this chapter. 


\subsection{Thermodynamic Properties of Liquid Mixtures}

Consider what happens when 2 liquids are mixed together. Are the liquids miscible (form a homogeneous mixture) or immiscible? To answer these questions it is important to introduce the thermodynamics of liquid mixtures.

To begin with it is important to define some terms which are measures of the composition of a mixture. The most important quantity, as far as the laws of mixing are concerned, is the mole fraction $x$. The mole fraction of a species $A$ is defined as the ratio of the number of moles af $A$ to the total number of moles present in the mixture. This is written:

$$
x_{A}=\frac{n_{A}}{\sum N} .
$$

It follows from equation 2.2 that the sum of the mole fractions of each species in a mixture is equal to unity.

\subsubsection{Gibbs Free Energy}

The Gibbs Free Energy $G$ of a system at any moment in time is defined as the enthalpy of the system $H$ minus the product of the temperature $T$ times the entropy of the system $S$,

$$
G=H-T S \text {. }
$$

$G$ therefore reflects the balance between the forces of enthalpy and entropy in a system.

The Gibbs Free Energy of the system is a state function because it is defined in terms of thermodynamic properties that are state functions. The change in the Gibbs free energy of the system that occurs during a reaction is therefore equal to the change in 
the enthalpy of the system minus the change in the product of the temperature times the entropy of the system:

$$
\triangle G=\triangle H-T \triangle S
$$

The enthalpy $H$ is a measure of heat content in the system where

$$
H=U+P V,
$$

where $U$ is the internal energy of the system, $P$ is the pressure and $V$ is the volume. The change in enthalpy of a system is therefore given by:

$$
\triangle H=\triangle U+P \triangle V
$$

A positive value of $\triangle H$ indicates an endothermic change i.e. heat is absorbed. $\mathrm{A}$ negative value of $\triangle H$ indicates an exothermic change, heat is released.

The entropy $S$ is a measure of the disorder in the system. The change in entropy $\triangle S$ indicates whether the system has become more disordered, larger value of $\triangle S$, or more ordered, smaller value of $\triangle S$.

The beauty of the equation defining the Gibbs free energy of a system (equation 2.4) is its ability to determine the relative importance of the $H$ and $S$ terms as driving forces behind a particular reaction. The change in free energy of the system that occurs measures the balance between the two driving forces that determine whether a reaction is spontaneous (Table 2.3). .

From the definition of the Gibbs free energy (equation 2.4), $\triangle G$ is negative for any reaction for which $\triangle H$ is negative and $\triangle S$ is positive. Therefore, $\triangle G$ is negative for any reaction which is favoured by both enthalpy and entropy terms. Any reaction 


\begin{tabular}{|c|c|}
\hline Favourable & Unfavourable \\
$\triangle H<0$ & $\triangle H>0$ \\
$\triangle S>0$ & $\triangle S<0$ \\
\hline
\end{tabular}

Table 2.3: Favourable and Unfavourable Enthalpy Change $\triangle H$ and Entropy Change $\triangle S$.

in which $\triangle G$ is negative is a favourable or spontaneous reaction. Conversely, any reaction in which $\triangle G$ is positive is an unfavourable or non-spontaneous reaction, i.e. it is difficult for this process to occur.

\subsubsection{Excess Functions}

A useful way of representing the thermodynamic behaviour of liquid mixtures is by the concept of excess functions. The excess Gibbs Free Energy change of mixing of a binary mixture is

$$
\triangle G^{E}=\triangle G_{\text {expt }}-\triangle G_{\text {ideal }},
$$

where $\triangle G_{\text {expt }}$ is the experimentally determined free energy of mixing of a real solution and $G_{i d e a l}$ is the free energy calculated on the basis of an ideal mixture. Ideal systems are defined as those for which intermolecular interactions (enthalpic contributions) are negligible. In ideal systems there are interactions, but the average A-B interactions in the mixture of liquid $\mathrm{A}$ and $\mathrm{B}$ are the same as the average $\mathrm{A}-\mathrm{A}$ and $\mathrm{B}-\mathrm{B}$ interactions in the pure liquids. Therefore the driving force for mixing is the increasing entropy of the systems as the molecules mingle and the enthalpy of mixing is zero. In ideal systems homogeneous mixing is always favoured where the properties of the solution are the same for all regions within the occupied volume. $G^{E}$ therefore measures the contribution of interactions between liquids $\mathrm{A}$ and B. Other thermodynamic excess functions, such as entropy and enthalpy, can be defined in a similar manner. 


\begin{tabular}{|c|c|c|c|c|c|c|}
\hline$H^{E}$ & $G^{E}$ & $T S^{E}$ & $V^{E}$ & $C_{p}^{E}$ & examples & phase behaviour \\
\hline+ & + & - & + & - & nitriles, ketones & $\begin{array}{l}\text { Complete miscibility or } \\
\text { upper-critical demixing }\end{array}$ \\
\hline+ or - & + & - & - & + & ethers, alcohols, amines & $\begin{array}{c}\text { Upper critical demixing, } \\
\text { tending to closed-loop }\end{array}$ \\
\hline- & + & - & - & + & tert-amines, & Closed-loop \\
\hline- & - & - & - & $\approx 0$ & urea, $\mathrm{H}_{2} \mathrm{O}_{2}$, polyols, sugars & Completely miscible \\
\hline
\end{tabular}

Table 2.4: Classification of solutes according to their Thermodynamic Excess Functions

Liquid mixtures can be classified according to the signs and magnitudes of their excess functions. For mixtures of chemically similar and symmetrical molecules $G^{E}$ is likely to be small, positive and symmetrical about $x=0.5$, where $x$ is the mole fraction of the solute species. For molecules of dissimilar sizes or where there are specific interactions, such as hydrogen bonds, the excess functions can be quite complex and water is an excellent example of this. Table 2.4 shows the classification of solutes according to their thermodynamic excess functions.

\subsubsection{Miscibility and Immiscibility}

If two liquids are placed in a container but separated by a partition, then when the partition is removed the liquids will mix. The liquids may mix spontaneously to form a homogeneous mixture. This results in a single phase, $P=1$ and the liquids are said to be miscible. On the other hand the liquids may not mix homogeneously and are said to be immiscible and have two phases, $P=2$.

For example, ethanol and water will form a single phase at room temperature whatever 
their relative proportions. Benzene and water when mixed will form two liquid layers ( 2 phases) unless one of the two components is present in large excess. Benzene has very low solubility in water and water has very low solubility in benzene so over most of the mole fraction range benzene and water are essentially immiscible.

From before, we know that $\triangle G_{m i x}$ must be negative to have a spontaneous homogeneous mixture. Remembering that

$$
\triangle G_{m i x}=\Delta H_{m i x}-T \triangle S_{m i x},
$$

then, this can be achieved by having $\triangle H_{m i x}$ negative and $\triangle S_{m i x}$ positive.

If both $\triangle H_{m i x}$ and $\triangle S_{m i x}$ are positive the liquids may be immiscible at low temperatures but miscible at high temperatures. The minimum temperature at which the liquids are miscible in all proportions is called the upper critical solution temperature (UCST). Below the UCST the liquids are in a two phase region $(\mathrm{P}=2)$, while above the UCST they are in a one phase region (P=1) (Figure 2.7(a)).

If both $\triangle H_{m i x}$ and $\triangle S_{m i x}$ are negative the liquids may be miscible at low temperatures and immiscible at high temperatures. The maximum temperature for which the system shows a single fluid phase $(\mathrm{P}=1)$ is called the lower critical solution temperature (LCST). Above LCST the liquids exist in a two fluid region $(\mathrm{P}=2)$ (Figure 2.7(b))

Since $\triangle H_{m i x}$ and $\triangle S_{m i x}$ are functions of temperature they may change sign as the temperature is varied and there are systems which exhibit both UCST and LCST. These systems have what is known as a closed loop immiscibility shown in Figure 2.7(c). Within the loop the fluids exist in a two fluid phase $(\mathrm{P}=2)$, while outside the loop they exist in a single fluid phase $(\mathrm{P}=1)$. Table 2.4 summarises and shows examples of these situations. 

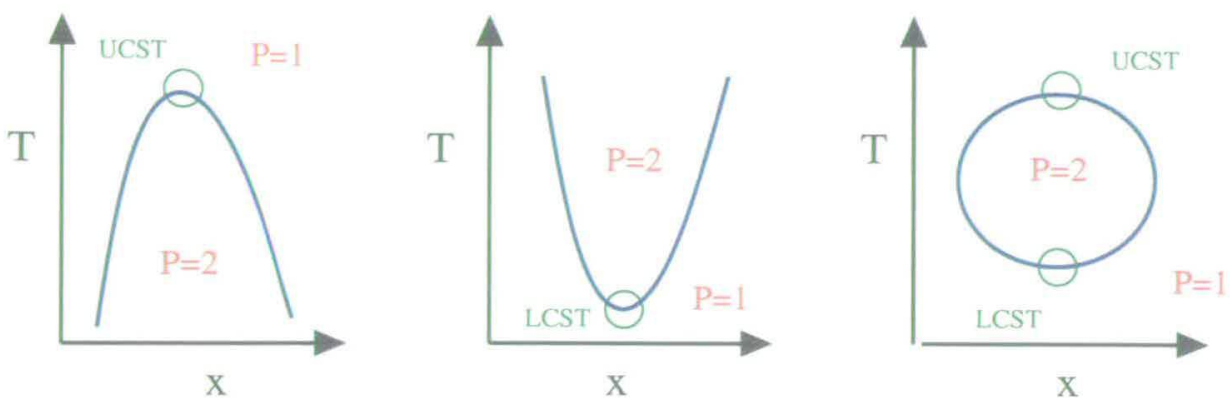

Figure 2.7: Schematic of miscible and immiscibility regions for a binary mixture with (a) Upper Critical Solution Temperature (UCST), (b)Lower Critical Solution Temperature (LCST) and (c) Closed Loop Immiscibility Region.

\subsection{Review of Current Understanding}

A wide range of experimental and simulation work has been carried out on aqueous alcohol systems. These 'simple' systems provide access to experimentally accessible areas which can be investigated in detail. In this section there will be a summary of the main findings from previous work.

\subsubsection{Neutron Diffraction of Aqueous Alcohols}

When a simple alcohol is mixed with water, the entropy of the system increases far less then expected for an ideal solution of randomly mixed molecules. In other words, the disorder of the system is not as much as might be expected. Pure water is conventionally regarded as a hydrogen bonded structure, with specific orientational (roughly tetrahedral) correlation between neighbouring molecules. It has been speculated that normal water structure is significantly enhanced by a hydrophobic entity, resulting in a more ordered (ice-like) structure near the headgroup. The loss of entropy associated with generating a 'cage' of water molecules around hydrophobic headgroups has been cited as the driving force for folding of proteins. One of the intriguing questions in the physics of aqueous solutions concerns the structure and dynamics of water cages around the hydrophobic headgroups in solution. 
But, is there any experimental evidence for this structural enhancement?

Neutron diffraction with comprehensive isotope substitution was used to study the molecular structure of a methanol-water $x=0.10$ mole fraction mixture[26]. This technique was used in the experiments carried out in this thesis and the method will be clearly explained in a following chapter.

These experiments aimed to study the structure of 'real' water around a dissolved methanol molecule using hydrogen isotope substitution to extract particular methanolwater correlation functions. The data gave the first direct experimental evidence that water molecules form a disordered cage around the methanol molecule. Evidence was found for the structure of hydration shells around the $\mathrm{CH}_{3}$ groups of the methanol molecule. The orientation of the water molecules around the $\mathrm{CH}_{3}$ group were tangential, much like that predicted by the standard model. However, further analysis suggested that the shell is achieved without a significant modification of the orientational order between water molecules which form a loose hydrogen bonded cage around the methanol molecule (Figure 2.8). Therefore, this work contradicted the speculation that water structure was enhanced by the methanol molecule.

Further work was carried out on methanol-water solutions in the dilute and concentrated regime. A methanol-water $x=0.05$ mole fraction was studied, also using the technique of neutron diffraction isotope substitution[13]. At this concentration, direct evidence was found for methanol association, with more than $80 \%$ of methanol molecules existing in clusters of 3 to 8 molecules. This non-polar association was thought to be a signature of the hydrophobic interaction. Figure 2.9 shows a simulation box containing methanol-water $x=0.05$ mole fraction solution[13]. Water and methanol oxygens are shown as white atoms. The methanol carbon atom is shown in black. Black bonds indicate methanol carbons in the first coordination shell. White bonds join all oxygen atoms. The snapshot of the simulation box reveals significant solute association with clusters of methanol molecules. Such cluster sizes are unex- 


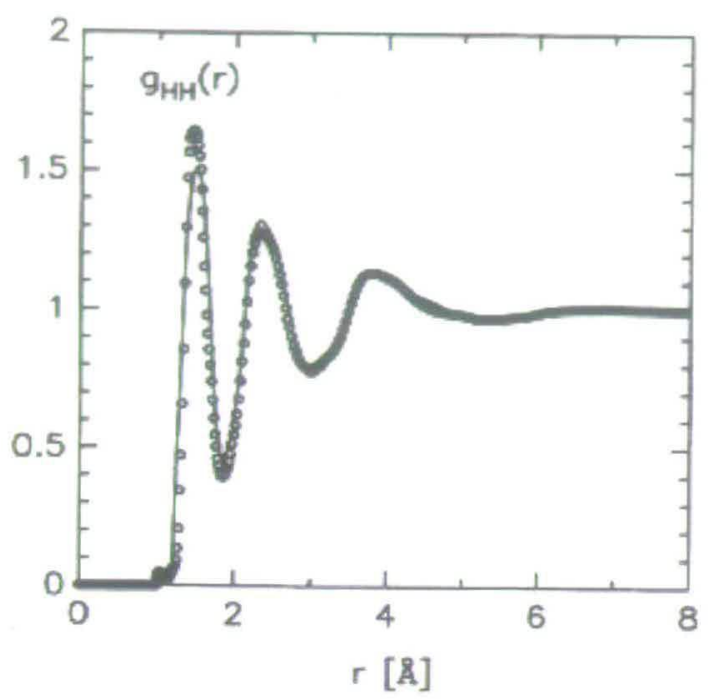

Figure 2.8: The hydrogen-hydrogen correlation function for a methanol-water $x=0.10$ mole fraction solution (line) compared to the same function for pure water (circles). The intramolecular peak at $r \sim 1.5 \AA$, the hydrogen bond peak at $r \sim 2.3 \AA$, and a third characteristic peak near $r \sim 3.8 \AA$ are all clearly visible in both cases[26]

pected on the basis of the accepted model of homogeneous mixing in this system. This solute association was also observed in a dilute tertiary butanol-water $x=0.06$ mole fraction system[27]. Contrary to previous studies, and from conventional views on hydrophobicity, no enhancement of water structure surrounding non-polar groups was observed.

Structural studies on other aqueous mixtures with solutes like tetraalkylammonium ions and tert-butanol have supported the case for unperturbed water structure[27], [28], [29]. Thus the methanol-water $x=0.05$ mole fraction mixture results provide confirmation of a perhaps initially unexpected effect. In this simple system, which has a much smaller non-polar headgroup, and the hydrophobic driving force is likely to be smaller, solute association is still evident.

The solute association seen at this concentration was further explored by performing experiments on a more concentrated solution, methanol-water $x=0.70$ mole fraction mixture. An increasing number of experimental and theoretical investigations suggested that hydrophobic headgroups in alcohol molecules cluster together. However, a 


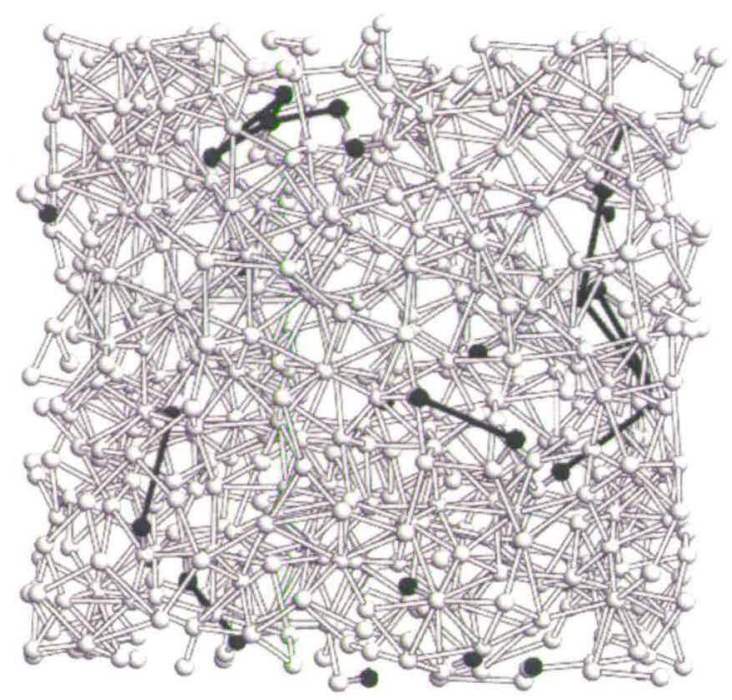

Figure 2.9: Snapshot of micro-segregation in a methanol-water $x=0.05$ mole fraction solution obtained from neutron diffraction and Empirical Potential Structural Refinement technique[13]. Water and methanol oxygens are shown as white atoms. The methanol carbon atom is shown in black. Black bonds indicate methanol carbons in the first coordination shell. White bonds join all oxygen atoms.

consistent description of the details of this self-association was lacking until a very important paper was published in 2002[12]. Neutron diffraction experiments were carried out to probe the molecular scale structure of a concentrated methanol-water mixture. Experiments indicated that most of the water molecules existed as small hydrogen bonded strings and clusters in a 'fluid' of close packed methyl groups[12]. The water molecules acted as bridges between neighbouring methanol hydroxyl groups through hydrogen bonding.

This work suggested that the anomalous thermodynamics of water-alcohol systems arises from incomplete mixing at the molecular level and from retention of remnants of 3-dimensional hydrogen bonded network structure of bulk water. Figure 2.10 shows a simulation box containing a methanol-water $x=0.70$ mole fraction mixture. Methanol methyl groups are shown as grey spheres. Large yellow spheres have been used to highlight the positions of water molecules and small red spheres denote methanol oxygen atoms. It is evident from this image that there is incomplete mixing at the molecular 


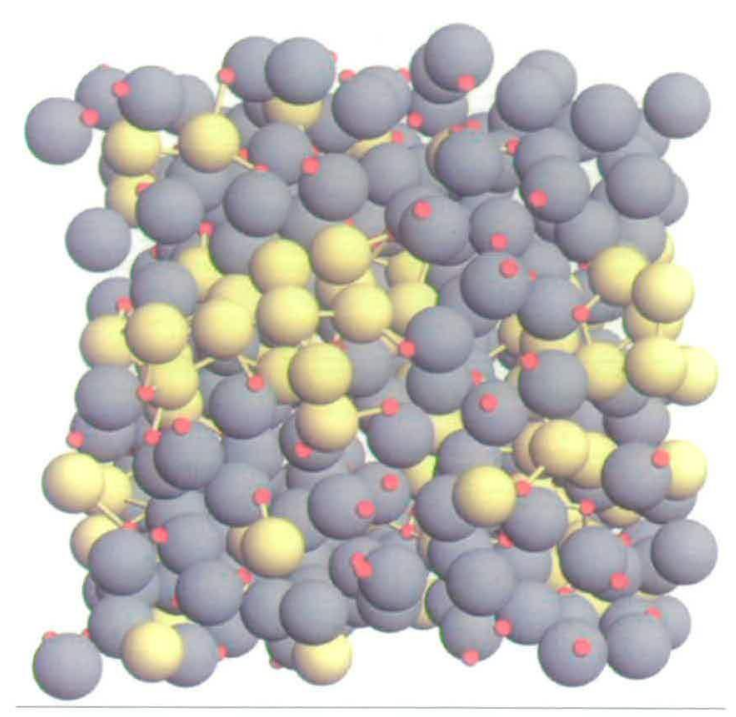

Figure 2.10: Micro-segregation in a methanol-water $x=0.70$ mole fraction system[12]. Methyl groups are shown as grey spheres. Large yellow spheres have been used to highlight the positions of water molecules and small red spheres denote methanol oxygen atoms. Yellow bonds join water oxygen atoms to other oxygen atoms within their first coordination shell.

level. The methanol-water solution is displaying micro-immiscibility.

\subsubsection{Computer Simulation}

Progress in the technology of fast computers has provided new techniques for probing the interactions and structures in liquids. The advantages of simulation methods include the potential to explore regions of phase space not readily accessible to experiment. For example, exploring the high pressure and low temperature regime of the complex water phase diagram. Simulations also provide graphic descriptions of molecular distributions and dynamics in liquids. The disadvantages are of course the size of the system in the simulation which is restricted by computer time and power. Also the output is essentially only as good as the input interaction potential. If a good model interaction potential is not known, then it is highly unlikely that we will learn anything real about our system.

The simulation of liquid water provides an excellent exemplification of both the power 
and the limitations of empirical potential simulations. As Guillot points out in an excellent recent review of the field [30] 'not a water model available in the literature is able to reproduce with a great accuracy all the water properties' And it is not for the want of trying; there are over 50 separate parameterisations for the water force field and many papers published annually devoted to the study of this unique and apparently simple liquid.

There are a huge number of publications of empirical simulations and ab initio of aqueous systems and these are beginning to address the issues of structure[31]. Early investigations by Okazaki et al[32] and Bolis et al[33, 32] used Monte Carlo methodologies and low or infinitely dilute concentrations of alcohol. Despite different computational models, and some apparent contradictions in their results, they all found an enhanced cage-like structure of water around the methyl group, in accordance with the Frank and Evans model. Later, Molecular Dynamics simulations explored other mixture compositions [34, 35] using effective potential models. Tanaka and Gubbins [36] were amongst the first to highlight the role of the water-water interactions in discussing aqueous solutions.

However, another early paper by Jorgensen et al[37] provided contradictory work to that of Okazaki[32] and Bolis[33]. According to Jorgensen, the main feature of the hydration in the water-rich region is the favourable solute-solvent hydrogen bonding. They found that the first coordination shell around the carbon of a methanol molecule contained 3.4 water molecules, which formed 2.3 hydrogen bonds with the methanol molecule, an average of 2.9 water-water hydrogen bonds per water molecule. Consequently, they formed 3.6 hydrogen bonds per water molecule, which was exactly the same as that for a water molecule in pure water. Although Okazaki et al[32] found an iceberg-like structure of water molecules around a methanol molecule, Jorgensen et al[37] did not observe a large distortion of the water molecules around the methyl group. 
Results supporting these findings were recently obtained by Fidler and Rodger[38] via molecular dynamics simulations. They found no evidence for enhanced structure around the hydrophobic end of alcohols up to $n$-butanol. Instead they found that the structure of water around the hydrophobic moiety of alcohol to be essentially the same as that found in pure water. In particular they found no evidence of the presence of a clathrate-like cage around the hydrophobic moiety of the alcohol. Some change in the water structure was found in the vicinity of the hydroxyl group of the alcohol, with a hydrogen bonded network closer to tetrahedral than that in pure water. They also found the dynamics of water, both translation and rotation, in the solvation shell of methanol to be modified.

Meng and Kollman [39] have performed MD simulations of various solutes including methanol using two different water models. The SPC/E model, a pairwise-additive potential, and the related POL3 model which includes non-additivity in the form of atomic polarisabilities. The system was of infinite dilution ( 1 methanol and 500 water molecules. They also found that the water structure around the hydrophobic groups is preserved rather than enhanced in the Frank-Evans 'iceberg' scenario. They also found that waters hydrogen bonding to polar solute groups sacrifice solvent-solvent hydrogen bonds to some degree. Laaksonen et al [40] have performed similar investigations of the water-methanol system across the entire composition range, thus permitting investigation of solute-solute association. They looked at the effect of alcohol on water structure and water on methanol structure. In the methanol-rich solutions they saw a high degree of ordering, characterised by a strong preference for tetrahedral arrangements, where the water molecules again appear must highly localised around the hydroxyl group of methanol. Strongly hydrated methanol molecules adopted specific relative positions in order to accommodate the ordering within their hydration cages. These very distinctive methanol-methanol correlations were evident at longer range, consistent with the suggestion that each methanol molecule is strongly solvated by a 'cage' of water molecules. 
The first study of liquid water using Ab Initio molecular dynamics was published in 1993. Since then the field has developed rapidly. Meijer et al[41] has published a number of papers on dilute aqueous mixtures. They studied a single methanol molecule in water (32 or 64 molecules in total). Their structural analysis indicated that the water structure was minimally perturbed by the presence of methanol, therefore providing no evidence of enhanced water structure. In a very recent paper Ruckenstein et al[42], $\mathrm{Ab}$ Initio quantum mechanical methods were used to examine clusters of methanol and water molecules. Their goal was to obtain information about the intermolecular interactions and the structure of methanol/water clusters at the molecular level. Their system consisted of 1 methanol molecule in 10-12 water molecules (dilute methanol system), and 1 water molecule in 10 methanol molecules (concentrated methanol system). It was found that the solvent molecules in both clusters (dilute methanol system and concentrated methanol system) could be sub-divided into two classes; not hydrogen bonded with central solute molecule and hydrogen bonded with central molecule (Figure 2.11). They stated that although these molecules were located at almost the same distance from the central solute molecule, they possessed very different intermolecular interaction energies with the central molecule. However, these systems sizes are extremely small and far from the complex reality of a real aqueous alcohol system.

Over the years there has been much work carried out on aqueous amphiphile systems using computer simulations. Findings from these studies have produced very contradictory ideas on the behaviour of these systems. Simulation work appears to provide evidence both for and against the traditionally-adopted model of water structure enhancement around the hydrophobic group. It is very clear that there is a lack of good experimental data to use as a benchmark to compare the findings from simulations. There is an obvious need to directly compare structural information from the results of experimental techniques with that from computer simulations. 
(a)

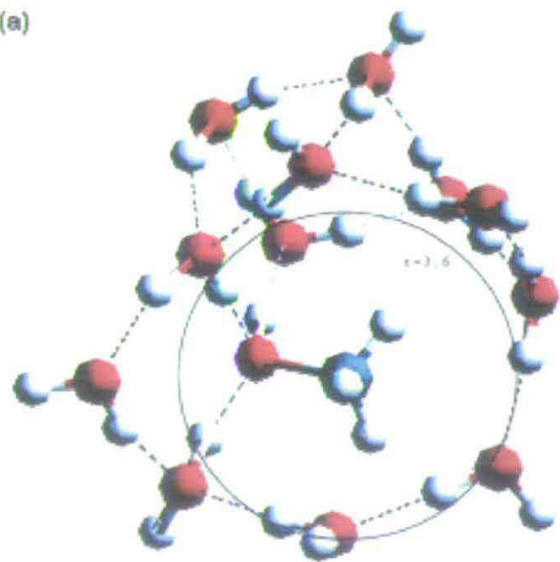

(b)

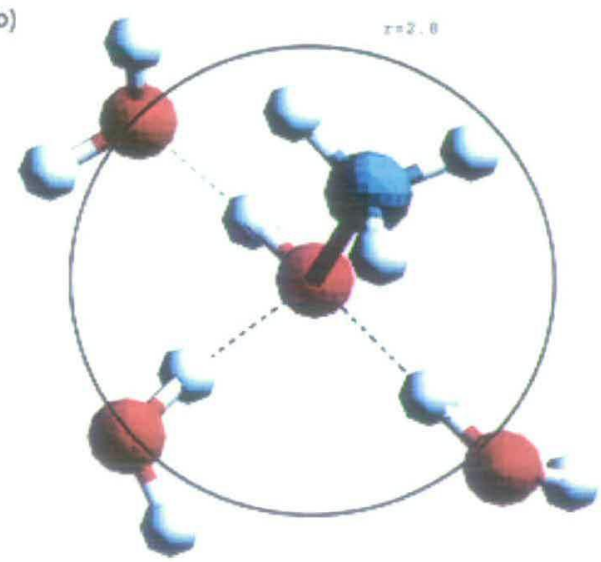

Figure 2.11: Optimised dilute methanol system, 1 methanol molecule and 12 water molecules. (a)The full cluster and (b)fragment containing only the central methanol molecule and the water molecules hydrogen bonded to the central methanol molecule, where - denotes hydrogen bonds[42] 


\section{Chapter 3}

\section{Probing Structure \& Dynamics}

\subsection{Motivation}

The technique of fluorescence spectroscopy was used to explore aqueous solutions of methanol. Using this powerful optical tool it was possible to probe a range of aqueous solutions at ambient temperature and under extreme conditions. The technique therefore provided an excellent opportunity to do preliminary investigations of the system over a huge range of state points. The technique was used to try and further explore the micro-segregated structure in methanol-water mixtures[13] and learn more about the dynamics of the system. The chapter begins with an introduction to the principles of fluorescence and the experimental setup. The results from a range of experiments are then presented.

\subsection{Principles of Fluorescence}

Fluorescence is used for studying the structure and dynamics of complex molecular systems. A molecule which is electronically excited returns to the ground state ei- 
ther by a radiative mechanism ${ }^{1}$ or non-radiative mechanism. The fluorescence photons have information about energy, time, polarisation and intensity (number of photons) at a given wavelength. Each of the parameters of the fluorescence photons gives information about the local environment surrounding the excited molecule (or fluorophore) under investigation. In this way, fluorescence intensity, polarisation and time dependence of fluorescence properties can be utilised as important parameters and used for characterisation.

The mechanisms by which electronically excited molecules come to the ground state are illustrated by a Jablonski diagram shown in Figure 3.1. The thick black horizontal lines represent electronic energy levels, while the thin black horizontal lines denote the various vibrational energy states (rotational energy states are ignored). Transitions between the states are illustrated as straight or dashed vertical lines, depending upon whether the transition is associated with absorption or emission of a photon (straight lines) or results from a molecular internal conversion or non-radiative relaxation process (dashed lines).

The diagram shows the ground state $\left(S_{0}\right)$, the first and second excited singlet states $\left(S_{1}\right.$ and $S_{2}$ ) and the excited triplet state $\left(T_{1}\right)$. In a singlet excited state the electron in the higher energy orbital has opposite spin orientation to the second electron in the lower orbital. The two electrons are said to be paired. In the triplet excited state the electrons are unpaired as their spins have the same orientation. Return to the ground state from the excited singlet state does not require an electron to change its spin orientation. A change in the spin orientation is needed for the triplet state to return to the singlet ground state. At each of these electronic energy levels the fluorophores can exist in a number of vibrational energy levels 0,12 etc. The transitions between the various electronic levels are vertical. This presentation is used to illustrate the instantaneous nature of light absorption. This process occurs in about $10^{-15} \mathrm{~s}$, a time too short for significant displacement of nuclei, an illustration of the Frank Condon principle (Figure

\footnotetext{
${ }^{1} \mathrm{~A}$ radiative mechanism is one where kinetic energy is converted into electromagnetic radiation
} 


\section{$\mathrm{S} 2$}

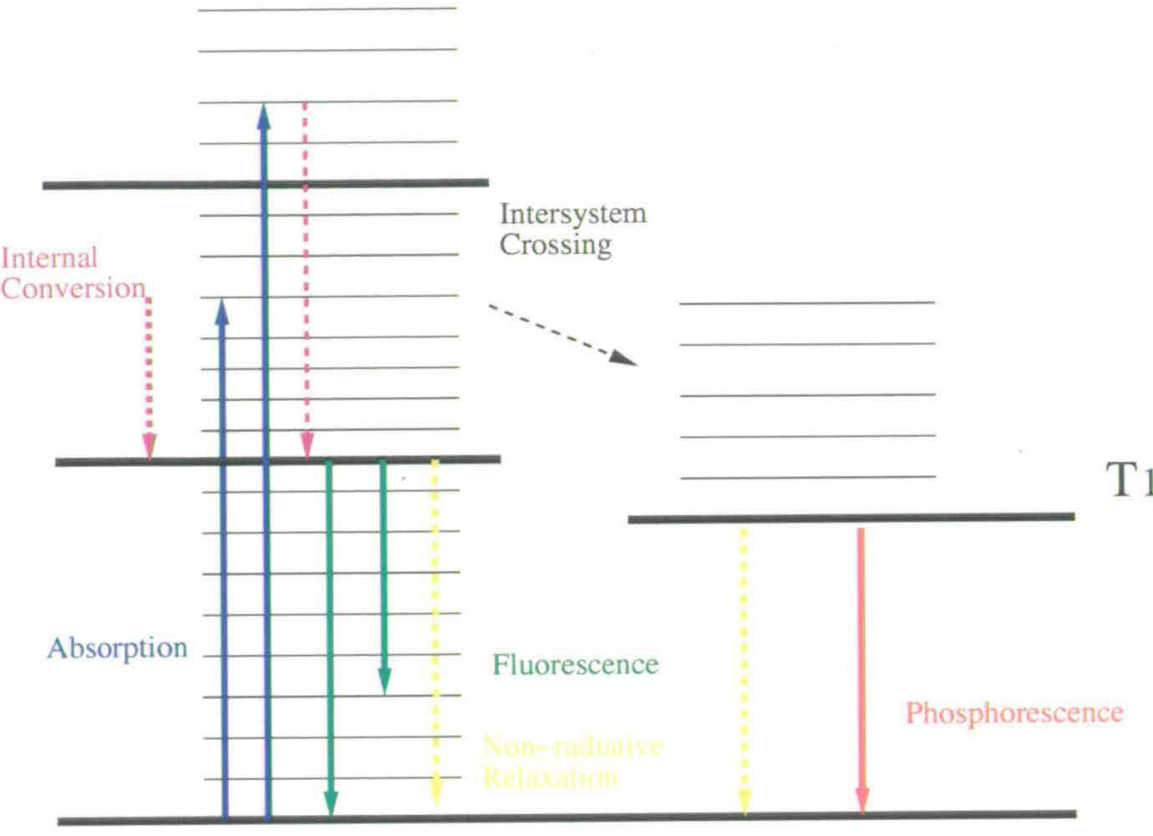

Figure 3.1: The Jablonski diagram showing the ground state $S_{0}$, first excited state $S_{1}$, second excited state $S_{2}$ and triplet excited state $T_{1}$

\section{$3.2)^{2}$.}

The absorption of a photon takes the molecule from the ground state (singlet state, $S_{0}$ ) to either the first excited state (singlet state, $S_{1}$ ) or the second excited state (singlet state, $S_{2}$ ). The excited molecule then relaxes to the lowest vibronic level of the first excited state through a process called internal conversion (IC). This process is very fast and due to this rapid relaxation, emission spectra are usually independent of excitation wavelength. From the lowest vibronic level it can relax from the singlet excited state to the ground state via three possible mechanisms (Figure 3.1). The first possibility is by fluorescence emission due to de-excitation of an electron from a singlet excited state (an allowed transition). Secondly, it can reach the ground state without emitting a photon, which is a non-radiative mechanism. Thirdly, it can go to a triplet state $\left(T_{1}\right)$ by a process called inter system crossing (ISC) which is also a non-radiative process. The electronic transition from the triplet $\left(T_{1}\right)$ to ground state is a forbidden transition and

\footnotetext{
${ }^{2}$ The nuclei are stationary during electronic transitions, and so excitation occurs to vibrationally excited levels of the excited electronic state
} 


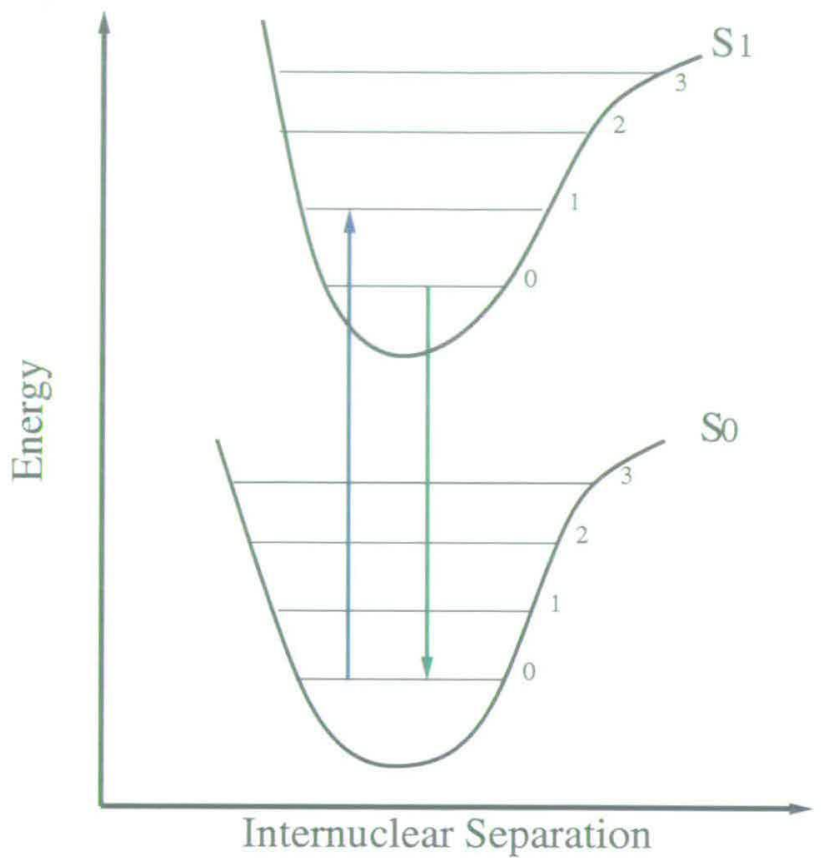

Figure 3.2: The Frank Condon energy diagram

hence is a very slow process relative to fluorescence excitation. The term forbidden transition refers only to the lowest order dipolar transition; higher order transitions are allowed but have much longer lifetimes. Emission from $T_{1}$ is called phosphorescence, and is generally shifted to much longer wavelengths relative to fluorescence. Although not indicated explicitly, a variety of other processes can influence the fluorescence emission; solvent effects, solvent relaxation, quenching and excited state reactions.[43]

Fluorescence-based techniques can be immensely useful in providing both microscopic and macroscopic-scale structural information, in addition to probing dynamical processes that occur on the timescale of fluorescence decay. Fluorescent molecules are often extremely sensitive to their local environment with many fluorophores being profoundly influenced by surrounding solvent molecules. The study of solvent-sensitive fluorescent molecules in heterogeneous solvent systems can potentially provide a very powerful means to probe both the underlying structure of the solvent molecules and also the kinetics of processes such as solvent exchange. 
In order to understand the potential of this technique it is necessary to introduce some of the important fluorescence spectroscopy observables. This will be done in the next section.

\subsection{Fluorescence Spectroscopy Observables}

\subsubsection{Excitation Process and Emission Process}

Structural information about the ground and excited state of molecules can be gained by laser induced fluorescence. An excitation experiment yields information on the excited state, while emission experiments yield information on the ground state.

The excitation process involves exciting a fluorophore with a range of different wavelengths and observing where the peak absorption occurs. Absorption occurs when the incident radiation matches a vibronic transition. It corresponds to moving to an excited state by absorbing a photon.

The emission process corresponds to decay back to the ground state by emission of a photon. A fluorescence emission spectrum is a plot of fluorescence intensity versus wavelength. The spacing between the observed bands gives the spacing between the vibrational levels in the ground state. The emission spectrum is characteristic of a fluorophore and sensitive to its local surrounding environment. In this way the emission spectrum can be used to probe the structure of the local environment of the fluorophore.

The emission spectra is shifted to longer wavelengths than the excitation spectra. This difference in wavelength is known as the Stokes' Shift and is a result of conservation of energy. Spectra of some compounds such as perylene, show significant structure due to the individual vibrational levels of the ground and excited states. Other compounds such as quinine show spectra devoid of any structure [43]. Therefore it important to know what to expect with the fluorophore you wish to utilise. 


\subsubsection{Fluorescence Intensity Decay and Lifetime}

The fluorescence intensity is a measure of the ability of a fluorescence molecule to decay through radiative $k_{r}$ or non-radiative $k_{n r}$ mechanisms. The fluorescence quantum yield $\phi$ is the probability with which an excited fluorophore emits a photon and is related to the radiative rate and non-radiative rate of the deactivation of the excited state as follows

$$
\begin{gathered}
\phi=\frac{k_{r}}{k_{r}+k_{n r}}, \\
k_{n r}=k_{I C}+k_{I S C}+k_{q}[Q],
\end{gathered}
$$

where $k_{n r}$ is the sum of $k_{I C}, k_{I S C}, k_{q}$, the rate constants for internal conversion, inter system crossing and quenching, respectively. Q denotes the quencher concentration. The quantum yield can be close to unity if the non-radiative rate of deactivation is much smaller than the radiative rate of fluorescence decay, $k_{n r} \ll k_{r}$.

Time resolved fluorescence methods give the kinetic information on the various processes involved in the deactivation of the excited state. With the advent of pulsed, mode-locked lasers as the excitation source, it is possible to have the time resolution of the order of femtoseconds for the excited processes under investigation. The typical fluorescence intensity decay is a plot of fluorescence intensity as a function of time (Figure 3.3). For a simple system having a single fluorophore and only one solvent environment the fluorescence intensity decay, $I(t)$, is a single exponential and is given as

$$
I(t)=I_{0} \exp \frac{-t}{\tau}
$$

where $I_{0}$ is the initial intensity and $\tau$ is the fluorescence lifetime. The fluorescence lifetime is related to the radiative and the non-radiative rates. It is defined by the 


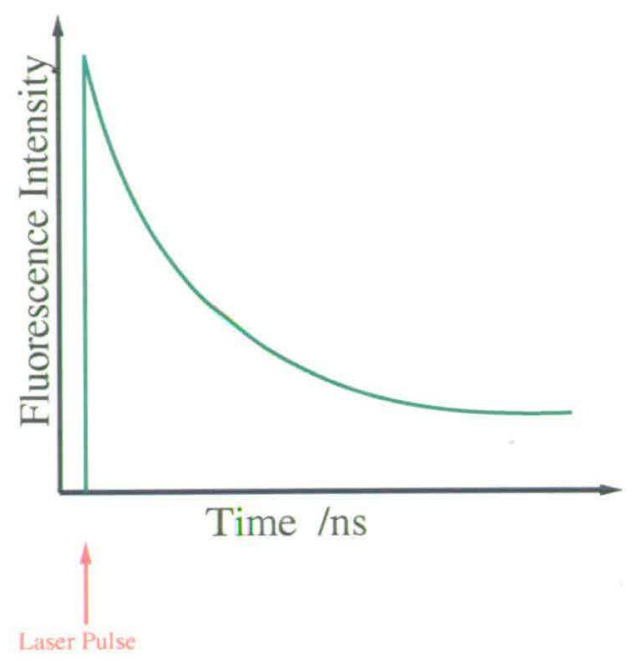

Figure 3.3: Illustration of Fluorescence Intensity Decay, showing fluorescence intensity decay as a function of fluorescence intensity with time

average time the molecule spends in the excited state, $S_{1}$, prior to return to ground state, $S_{0}$.

$$
\tau=\frac{1}{k_{r}+k_{n r}} .
$$

The fluorescence quantum yield of the excited state, mentioned earlier, is related to the fluorescence lifetime in the following way

$$
\tau=\frac{\phi}{k_{r}}
$$

The fluorescence emission is a random process, and few molecules emit their photons at $t=\tau$. The fluorescence lifetime is therefore an average value of the time spent in the excited state. Molecules with fluorescence spectra, quantum yields and fluorescence lifetimes that are sensitive to their environment can be used as probes for structural studies of biological macromolecules and mixtures of solutions. The basic information contained in fluorescence measurements relates to the molecular environment around the fluorescence probe. The fluorescence reflects various interactions of the probe with 
the surrounding molecules, especially those present during its excited state, and carries information about the rotations and displacements of the fluorescence probe molecules caused by Brownian thermal motion.

Generally, complex systems may have multiple environments for the fluorophore and hence more than one fluorescence lifetime. The fluorescence intensity decay $I(t)$ will no longer be fitted by a simple exponential function. Instead, a multi-exponential function is needed and the fluorescence intensity decay can be written:

$$
I(t)=\sum_{i=1}^{n} \alpha_{i} e^{\frac{-t}{\tau_{i}}},
$$

where $\alpha_{i}$ and $\tau_{i}$ are the ith pre-exponential factors (amplitude) and the fluorescence lifetime in the multi-exponential decay respectively. Pre-exponential factors are generally positive but can be negative whenever there is excited state kinetics. In the case of multi exponential decays the average lifetime, which is proportional to the total area under the fluorescence decay curve is defined as:

$$
\tau_{a v}=\frac{\sum_{i} \alpha_{i} \tau_{i}}{\sum_{i} \alpha_{i}} .
$$

\subsection{Experimental Procedure}

In this section all the experimental procedures for the fluorescence spectroscopy investigations will be introduced and explained.

\subsubsection{The Experimental Probe}

The fluorescence probe used for this experiment was 1 - anilinonaphthalene - 8 sulfonic acid (ANS). The molecular structure of ANS is shown in Figure 3.4. 


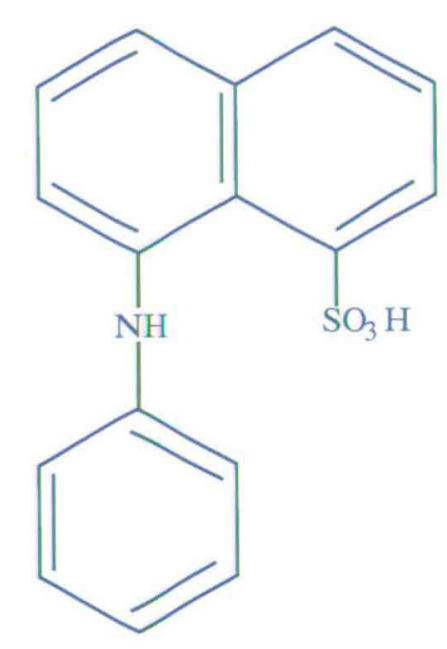

Figure 3.4: Molecular structure of 1 - anilinonaphthalene $-8-$ sulfonic acid (ANS). The fluorescence probe

The fluorescence spectrum of ANS in solution depends strongly on the nature of the solvent, especially on its polarity. ANS shows a dramatic decrease in fluorescence quantum yield $\phi$ when the solvent is changed from methanol $(\phi=0.17)$ to water ( $\phi$ $=0.003)$. Accompanying the decrease in $\phi$ is a large red shift in the fluorescence emission maximum $\lambda_{e m}^{\max }$. The fluorescence lifetime of ANS in methanol is known to be 6000 ps. The fluorescence lifetime of ANS in water is known to be much shorter, 250 ps[44]. An ANS molecule surrounded mainly by water molecules will have a fluorescence lifetime shorter by a factor of nearly 24 than one surrounded mainly by methanol molecules. The ANS molecule can therefore act as a probe for local solvent structure.

\subsubsection{Sample Preparation}

All fluorescence spectroscopy measurements were made with the ammonium salt of ANS (Fluka, used as received). The methanol used in this study was spectroscopic grade from Fluka and was used as received. The water was HPLC grade (Aldrich) and was used as received. The solvents were stored in clean glass containers and 
sealed with parafilm to avoid contamination and evaporation. Solutions of ANS were prepared from a stock solution and stored in the dark. A stock solution of ANS in methanol was prepared at a concentration of $10^{-4} M$. The dilution factor was considered allowing samples of volume $2 \mathrm{ml}$ and concentration $10^{-6} \mathrm{M}$ to be prepared in agreement with previous work.[45] If using a stronger concentration of ANS a dimer may form (excited 2 molecule state). The lifetime of ANS fluorescence at room temperature was used as a routine check of sample purity after cooling and after storage. No emission could be detected from the solvents under the instrumental conditions employed.

A number of different concentrations of methanol-water solutions were prepared using clean pipettes for each solvent. For the fluorescence experiment the sample was held in a $1 \mathrm{~cm}-1 \mathrm{~cm}$ glass cuvette which was sealed with a plastic lid. The cuvette was thoroughly cleaned using acetone, rinsed with HPLC grade water and then placed in an oven to dry. This ensured that any contaminants from previous experiments were removed. The same cuvette was used throughout the experiment to ensure direct comparison between results. The cuvette was further sealed with parafilm to reduce the rate of evaporation of the sample.

\subsubsection{Absorption and Emission Measurements}

Absorption spectroscopy is a spectroscopic tool which provides the wavelength of transition and the corresponding molar extinction coefficient $\epsilon_{\lambda}$ of a fluorophore under investigation[43]. Absorption measurements were recorded on a Cary 50 spectrometer. Absorption spectra give information about the energy of the transitions of the absorbing molecule. The absorbance $A$ of an absorber of concentration $C$, having a molar extinction coefficient $\epsilon_{\lambda}$ at wavelength $\lambda$ is given by the equation

$$
A=\log \frac{I_{0}}{I}=\epsilon_{\lambda} C l,
$$


where $I_{0}$ and $I$ are the intensities of the incident and transmitted light respectively and $l$ is the path length of the light beam passing through the sample. The sample of ANS and solvent is contained in a quartz cuvette with path length $1 \mathrm{~cm}$. Concentrations were determined using equation 3.8 and using the molar extinction coefficient $\epsilon_{\lambda}$ at wavelength $\lambda$. The absorbance of solutions was between 0.1-0.2 at the excitation wavelength.

The emission measurements were carried out on an Edinburgh Instruments picosecond fluorescence spectrometer. The design and setup of this instrument will be explained in a later section. For the emission process the laser is fixed at one particular excitation wavelength. The laser excitation results in emitted fluorescence from the fluorescence molecule in the solution and is dispersed into component wavelengths by a monochromator. The emission spectrum is eharacteristic of a fluorophore and sensitive to its local surrounding environment.

\subsubsection{Time Resolved Fluorescence Measurements}

Time resolved fluorescence measurements were carried out using a high power analysis setup. A schematic of the complete experimental arrangement on the optical bench is shown in Figure 3.5. The samples were measured in a $1 \mathrm{~cm}$ pathlength cuvette in an Edinburgh Instruments picosecond fluorescence spectrometer. The spectrometer was a modular, computer controlled L-geometry spectrometer for measuring fluorescence lifetimes based on the technique of Time Correlated Single Photon Counting. An optical trigger module was used in the alignment to act as a starter for the lifetime measurements. A monochromator was used as a detector. The design and operation of this equipment has been described in more detail in other publications[46]. The 2nd chamber of the spectrometer was used allowing space for the development and addition of a low-temperature control system, which will be described in detail. The excitation source was a ultrafast mode locked Ti-Sapphire femtosecond laser system 


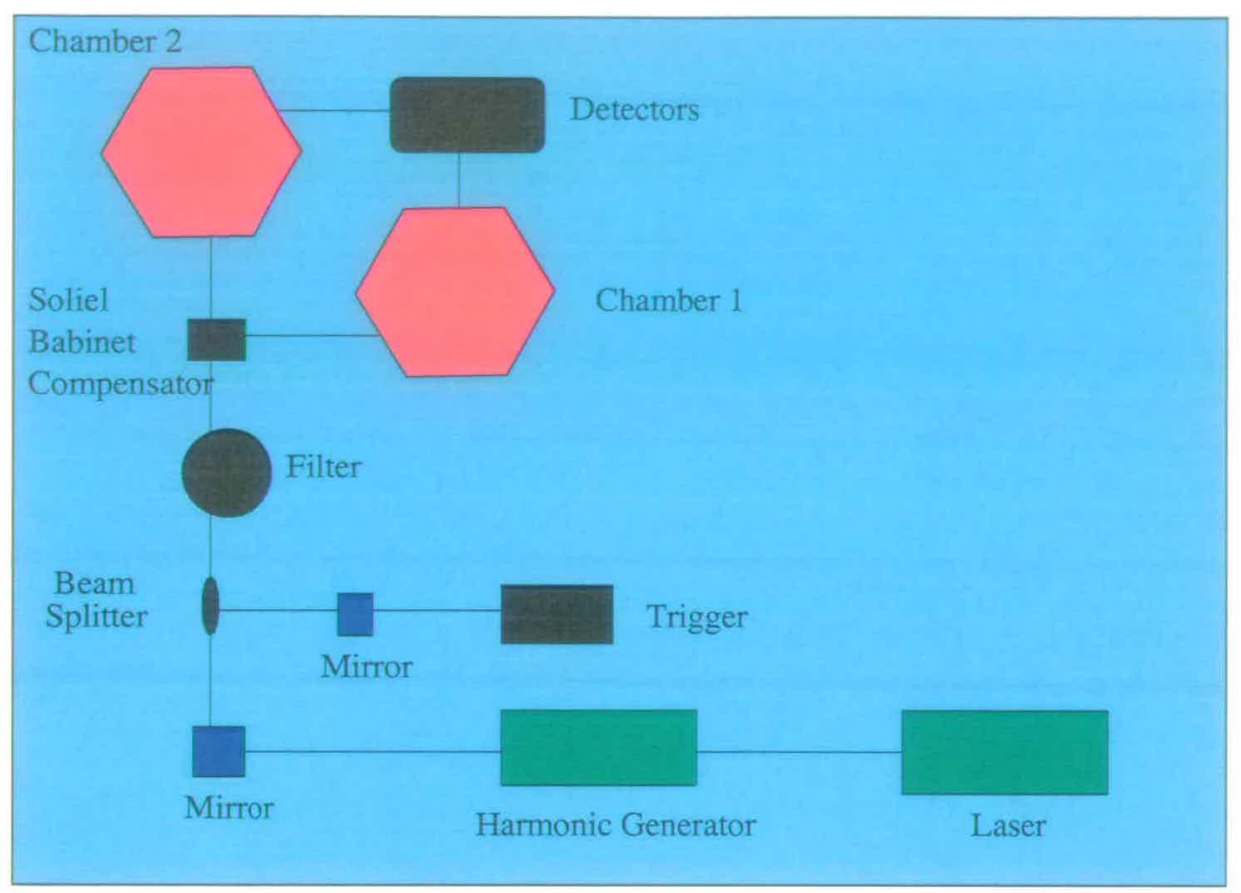

Figure 3.5: Schematic of Fluorescence Spectrometer Set-up on Optical Bench

from Coherent (10 W Verdi and Mira Ti-Sapphire laser) producing pulses of $200 \mathrm{fs}$ at $76 \mathrm{MHz}$. The output of the Mira was passed through a pulse picker (reducing the repetition rate to $4.75 \mathrm{MHz}$ ) and then frequency doubled to give an output at $395 \mathrm{~nm}$. The laser produced short pulses, with fixed intervals, providing high power analysis of solutions.

The excitation beam was split, and one portion was used to trigger a fast photodiode. The emission from the sample was collected at right angles to the excitation direction and at the magic angle with respect to the vertical polarisation of the incident beam. This allows elimination of polarisation bias. Excitation polarisation was controlled by a Soleit-Babinet compensator (Figure 3.5). The Soleil Babinet Compensator device modifies the orientation of linear polarised laser light required for fluorescence anisotropy studies. The light was passed through a monochromator (bandpass $10 \mathrm{~nm}$ ) then detected by a Hamamatsu MCP-PMT (R3809U-50). Decay curves were recorded with 4096 channels and to 10,000 counts in the peak channel on 5,20 and 50 ns ranges. 
The quality of the fits was determined by the value of the reduced chi-squared statistical parameter and by visual inspection of residuals. This analysis technique will be explained in more detail in the following section. The instrument response function (IRF) of the system was measured using a very dilute solution of Ludox scatterer. The full width at half maximum (FWHM) was approximately 50 ps.

Time-resolved fluorescence spectroscopy was performed using the technique of time correlated single photon counting (TCSPC)[23]. TCSPC (Figure 3.6) is a well established and accurate technique for fluorescence lifetime measurements. The principle of TCSPC is the detection of single photons and the measurement of their arrival times in respect to a reference signal, the light source. TCSPC is a statistical method and a high repetitive light source, in this case a Titanium-Sapphire laser, is needed to accumulate a sufficient number of photon events for a required statistical data precision. The lifetimes were extracted from the data by iterative convolution analysis using the F900 software provided by Edinburgh Instruments[46]. The electronics of the TCSPC can be compared to a fast stopwatch with two inputs (Figure 3.6). The clock is started by the START signal pulse and stopped by the STOP signal pulse. The time measured for one sequence is represented by an increase of a memory value in a histogram, where the channels on the $\mathrm{x}$-axis represent time. The resulting histogram counts versus channels represent the fluorescence intensity versus time (Figure 3.7). The TCSPC technique is widely accepted to be the method of choice for maximum sensitivity, dynamic range, accuracy and precision.

\subsubsection{Analysis of Fluorescence Intensity Decays}

The experimentally measured time resolved fluorescence intensity $F(t)$ was analysed by fitting it to a function with the sum of exponentials and which is now described[47]. The observed time resolved fluorescence $F(t)$ is not the true fluorescence response function $I(t)$ from the sample under investigation but is a convolution of the instrument 


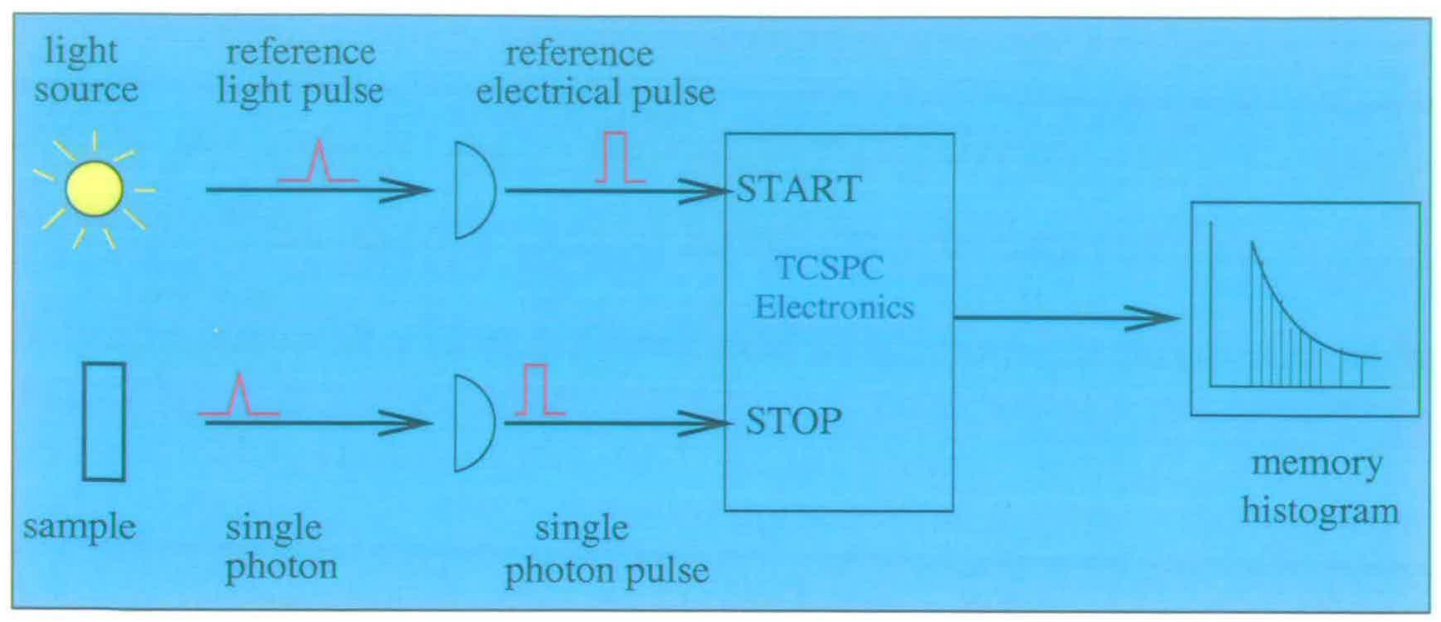

Figure 3.6: Time Correlated Single Photon Counting

response function $R(t)$ and $I(t)$ and is given by

$$
F(t)=\int_{0}^{t} R(s+\delta) I(t-s) d s,
$$

in which $\delta$ is the shift parameter. The shift is necessary because the IRF is measured at a wavelength near to the excitation wavelength whereas the fluorescence is detected at a wavelength far from the excitation. As the time response of the detector (photomultiplier) depends on wavelength (due to the wavelength dependence of the energies of the electrons ejected from the photocathode), the arrival of the signal at the TAC from the MCP or PMT is wavelength dependent and hence the shift parameter is needed in the analysis.

Iterative reconvolution analysis was used to determine the pre-exponential factors $\alpha_{i}$ and lifetimes $\tau_{i}$ for the fluorescence decay curves (Figure 3.7). Discrete component analysis was performed with F900 software[46], whilst global analyses were performed using FAST software (Alango Ltd.). The fractional intensities $\left(f_{i}\right)$ were calculated from the values of lifetimes $\left(\tau_{i}\right)$ and pre-exponential factors $\left(\alpha_{i}\right)$ as follows: 


$$
\begin{aligned}
& f_{1}=\frac{\alpha_{1} \tau_{1}}{\alpha_{1} \tau_{1}+\alpha_{2} \tau_{2}}, \\
& f_{2}=\frac{\alpha_{2} \tau_{2}}{\alpha_{1} \tau_{1}+\alpha_{2} \tau_{2}} .
\end{aligned}
$$

Non-linear least-squares method ${ }^{3}$ was used for obtaining $\alpha_{i}$ and $\tau_{i}$. In the iterative reconvolution method the parameters $\alpha_{i}$ and $\tau_{i}$ were adjusted iteratively such that the convergence was attained. The goodness of the fits were judged by the reduced $\chi^{2}$ value which should be close to $1.0[48]$. The reduced $\chi^{2}$ is defined as

$$
\chi^{2}=\frac{1}{n-1} \sum_{1=1}^{n} \frac{\left(F_{e}\left(t_{i}\right)-F_{c}\left(t_{i}\right)\right)^{2}}{\sigma_{i}^{2}} \approx 1.0,
$$

where $F_{e}\left(t_{i}\right)$ is the experimental value of time resolved fluorescence intensity at time $t_{i}, F_{c}\left(t_{i}\right)$ is the calculated value of the time resolved fluorescence intensity at time $t_{i}$, $n$ is the number of data points fitted, $l$ is the number of free parameters in the analysis and $\sigma_{i}$ is the standard deviation associated with the ith data point and is defined

$$
\sigma_{i}=\sqrt{F_{e}\left(t_{i}\right)},
$$

and is based on Poisson statistics.

The goodness of fit is also judged by the randomness of the weighted residuals of the experimental data with that of the calculated data (Figure 3.7). The weighted residuals $r\left(t_{i}\right)$ of the data are defined as

$$
r\left(t_{i}\right)=\frac{F_{e}\left(t_{i}\right)-F_{c}\left(t_{i}\right)}{\sigma_{i}} .
$$

For a best fitted experimental data the weighted residuals $r\left(t_{i}\right)$ should be distributed randomly about zero (red line on Figure 3.7).

\footnotetext{
${ }^{3}$ Levenberg-Marquardt (also called Marquardt method) was adopted for the non-linear analysis
} 


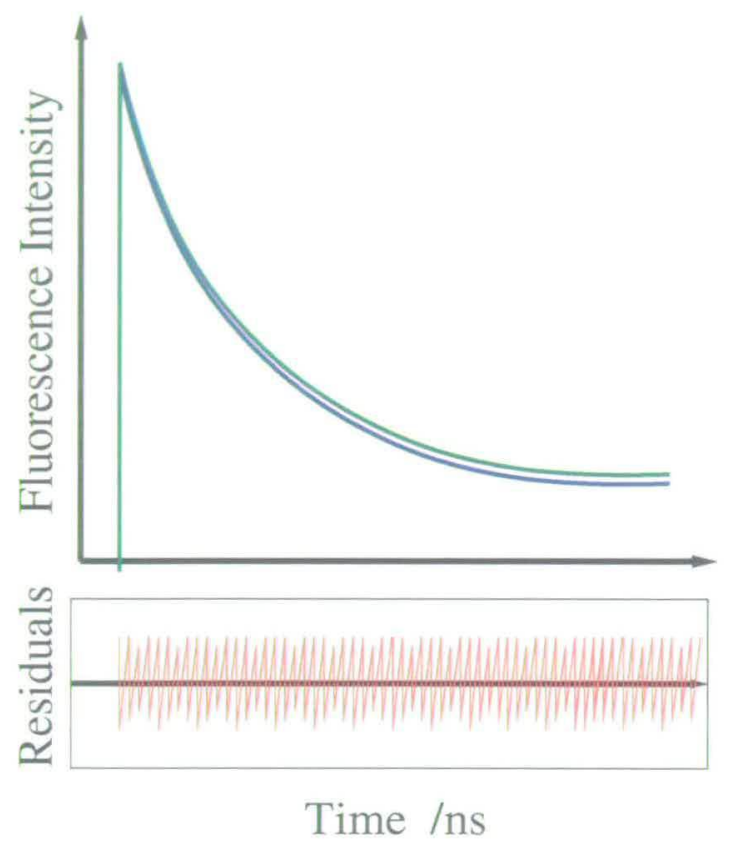

Figure 3.7: Illustration of Exponential fit (blue line) to a Fluorescence Intensity Decay (green line) of a fluorophore in solution and residuals (red line) indicating the goodness of fit of data.

\subsubsection{Low Temperature Experimental Setup}

To explore the behaviour of the aqueous system under the conditions of cooling an extensive set of experiments were carried out on the methanol-water system. The motivation was to understand how the dynamics of the system changes and learn more about the structure of the micro-segregated solution. The low temperature setup was designed by Hugh Vass.

For temperature dependent measurements, a copper holding cell was designed to securely hold the sample cuvette in place in the spectrometer chamber. Windows in the copper cell were positioned in the chamber to give optimum signal for the sample with right-angle detection geometry. The temperature was controlled and adjusted with a flow of cold nitrogen gas through copper tubing around the copper cell. Temperatures were measured with a thermocouple placed directly on the sample cuvette. The copper cell was enclosed in a blackened polystyrene case to provide both insulation and to avoid stray reflections. 
The temperature controller is used to establish the current temperature. To stabilise the system and reach a required temperature, the heater is switched on which uses the thermocouple on the copper holder to warm up the cuvette. The stability of the temperature controller was within $\sim 0.1 \%$ of the desired temperature. Both the liquid nitrogen and the heater can be used to reach a particular temperature and stabilise the system for measurements. For the lower temperatures the power meter was increased slowly allowing small scale decrease in temperature of the cuvette, avoiding condensation forming on the cuvette and causing reflections. 


\begin{tabular}{|l|c|c|}
\hline $\begin{array}{l}\text { Concentration } \\
\mathrm{x}\end{array}$ & $\begin{array}{c}\text { Methanol nm } \\
\lambda_{e m}^{\max }\end{array}$ & $\begin{array}{c}\text { Ethanol nm } \\
\lambda_{e m}^{\max }\end{array}$ \\
\hline 1.0 & 485 & 464 \\
\hline 0.7 & 490 & 485 \\
\hline 0.5 & 498 & 490 \\
\hline 0.27 & 510 & 503 \\
\hline 0.10 & 514 & 512 \\
\hline 0.00 & 525 & 523 \\
\hline
\end{tabular}

Table 3.1: Fluorescence emission maximum in relation to concentration for methanol-water solutions from experimental analysis and for ethanol-water solutions from literature[45].

\subsection{Results}

\subsubsection{Behaviour of the Probe}

A marked solvent dependence on the fluorescence emission maximum with concentration in a methanol-water solution was observed (Table 3.1). A shift of $40 \mathrm{~nm}$ occurred between ANS in pure water $\left(\lambda_{e m}^{\max }=525 \mathrm{~nm}\right)$ and ANS in pure methanol $\left(\lambda_{e m}^{\max }=\right.$ $485 \mathrm{~nm}$ ). This shift is highly nonlinear with changes in solvent concentration in the mixed solvents in agreement with work done by Robinson et al[45] on ethanol-water solutions at different concentrations (Table 3.1).

In low viscosity solvents the position of the fluorescence maximum depends in a simple way on the polarity of the solution only. The polarity of water is $94.6 \mathrm{Z}$, and the polarity of methanol is $83.6 \mathrm{Z}[49]$ ). From Table 3.1 it is evident that the higher the proportion of methanol then the lower the polarity and the shorter the fluorescence emission maximum $\lambda_{e m}^{\max }$ (i.e. the bluer the emission). As more water is added to the mixture, the polarity of the solution increases and $\lambda_{e m}^{\max }$ becomes longer (i.e. the redder the emission). The polarity and quantum yields of a range of alcohols are displayed 


\begin{tabular}{|l|c|c|c|}
\hline Solvent & Quantum Yield & $\begin{array}{c}\text { Polarity } \\
\mathrm{Z}\end{array}$ & $\begin{array}{c}\lambda_{e m}^{m a x} \\
\mathrm{~nm}\end{array}$ \\
\hline octanol & 0.646 & 75.0 & 465 \\
\hline butanol & 0.516 & 77.7 & 477 \\
\hline propanol & 0.476 & 78.3 & 479 \\
\hline ethanol & 0.361 & 79.6 & 480 \\
\hline methanol & 0.17 & 83.6 & 485 \\
\hline ethylene glycol & 0.12 & 85.1 & 495 \\
\hline water & 0.0032 & 94.6 & 520 \\
\hline
\end{tabular}

Table 3.2: The fluorescence emission maximum $\lambda_{e m}^{\max }$ for a number of different alcohols and for water, along with their polarity and quantum yield[44]

in Table 3.2. The increase in quantum yield is accompanied by a blue shift of fluorescence maximum as the polarity of the solvent decreases. The fluorescence emission is dramatically quenched in more polar solvents.

Part of the mystery of the ANS emission spectrum has concerned the exact nature of the solute-solvent interaction in the excited state, and there are a number of different theories. Kosower et al.[50] contend that the spectral shifts arise because of the chargetransfer nature of the emitting state. ANS is known[50] to undergo charge transfer (CT) from one aromatic moiety to the other ring and solvation. In steady state, in non-polar solvents, the emission is strong and is mostly from the locally excited state, before charge separation. In polar solvents, the fluorescence quantum yield decreases and is dominated by emission from the $\mathrm{CT}$ state. The solvent polarity and rigidity determine the wavelength and yield of emission, making ANS a very useful biological probe.

On the other hand, hydrogen-bonding interactions may be involved. As the proportion of water molecules in the solvent is increased, extra hydrogen-bonding stabilisation of the excited state may contribute to the fluorescence red-shift. Brand et al[51], using 
the theories of solvent reorientation due to Lippert[52] and Bakhshiev[53], interpret the fluorescence shifts in terms of a large change of dipole moment between the ground and excited states.

\subsubsection{Structure of the System}

Under ambient conditions, the fluorescence lifetime decay of the ANS fluorescence probe was investigated for both water and methanol. The pure solvents were found to be single exponential in pure water and pure methanol and the lifetimes were in good agreement with literature values[49]. The fluorescence lifetime of ANS in methanol was found to be $6.09 \mathrm{~ns}$ with $\chi^{2}=1.06$ while the fluorescence lifetime of ANS in water was found to be $0.24 \mathrm{~ns}$ with $\chi^{2}=1.09$. The fluorescence decay curves are shown to illustrate the excellent fit to the experimental data (Figure 3.8). The green line shows the fluorescence decay of ANS in the solvent, the black line shows the instrument response function (IRF), the blue line shows the reconvolution tail fit to the data and the red line below shows the residuals. For the ANS in water system, only a small portion of the decay was fitted, illustrated by the blue line, due to the influence of the instrument response in this extremely fast fluorescence decay.

The fluorescence lifetime measurements of ANS in a number of methanol-water solutions, across the concentration range, were then investigated. The fluorescence lifetimes calculated for each solution are plotted in Figure 3.9. For all solutions, the fluorescence intensity decay could be accurately fitted with a single exponential. Figure 3.9 also shows the relationship between fluorescence lifetime and concentration of aqueous solution for ethanol and acetonitrile from work carried out be Ebbesen et al[54]. The relation is highly nonlinear as the concentration of solute is increased. Interestingly, it is evident that the fluorescence lifetime at all intermediate concentrations lies below linear expectations i.e. the fluorescence lifetime is always faster than what would be expected if there was a linear relationship between the fluorescence life- 

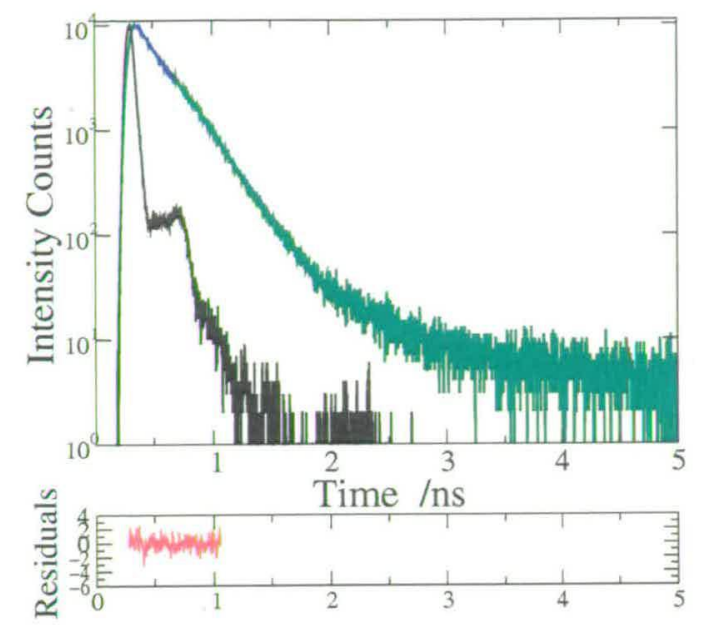
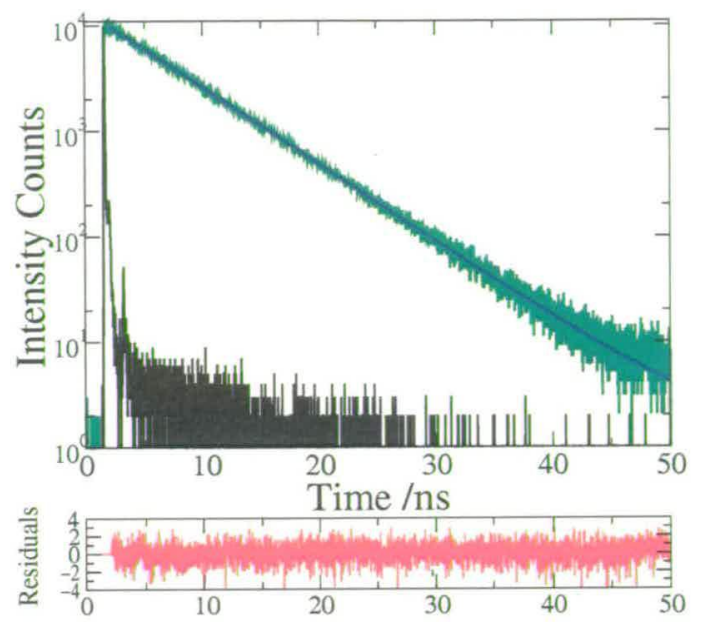

Figure 3.8: Time Resolved Fluorescence Intensity Decays for ANS in water (left) and ANS in methanol (right)

time of ANS in pure water and pure methanol. These results suggest that on average the ANS molecule prefers to be in the water environment in these different solutions. Therefore the ANS molecule would appear to prefer to be in a more polar environment. To improve the resolution of a fluorescence lifetime measurement it is necessary to perform measurements at additional wavelengths. This is known as a global analysis. There may be difficulties in resolving decay times and amplitudes in a solution which might need a multi-exponential fit to the decay. The basic idea of global analysis is to combine a number of fluorescence experiments in which some of the parameters are the same in all measurements and some are different. A global experiment would be to measure the fluorescence intensity decays at a number of different wavelengths. The multiple intensity decay curves are then analysed simultaneously to recover the fluorescence lifetime values $\tau_{i}$. The $\tau_{i}$ values are assumed to be independent of emission wavelength. In the case of global analysis, the calculation of $\chi^{2}$ now extends over several data sets. For the fitted functions the pre-exponential factors $\alpha_{i}$ and fractional intensities $f_{i}$ are different for each wavelength because of the different relative contributions of the fluorescent probe.

The fluorescence decay for ANS in a $\mathrm{x}=0.5$ mole fraction methanol-water solution 


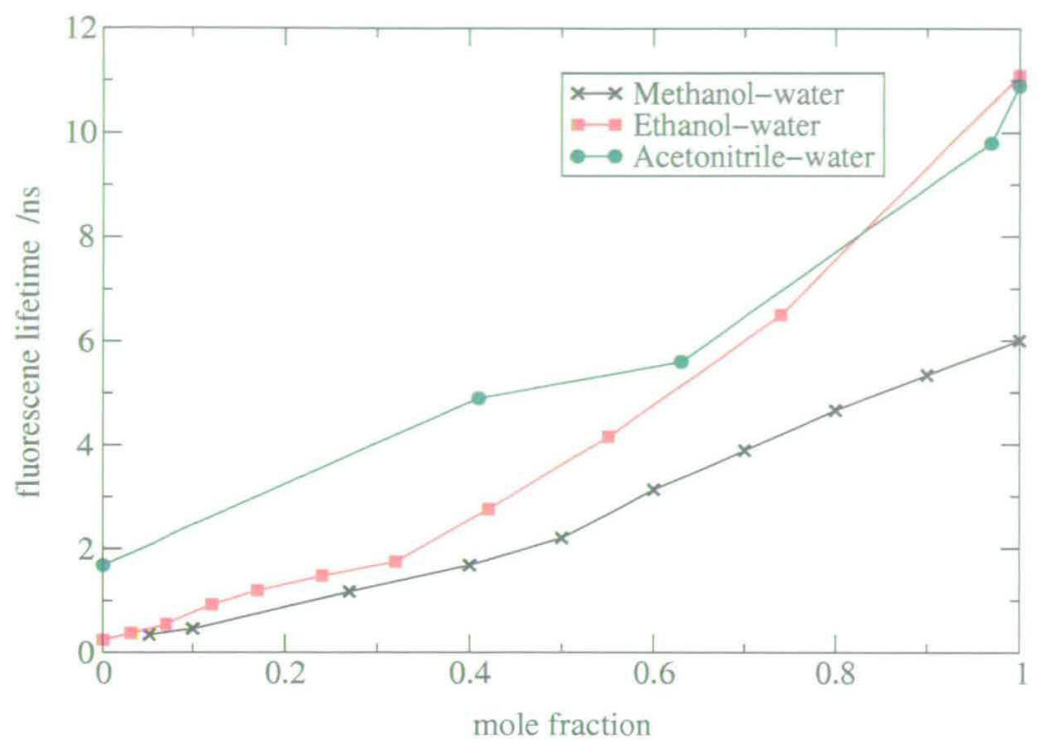

Figure 3.9: The fluorescence lifetime of 1,8-anilinonaphthalene sulfonate ANS in methanolwater mixtures, ethanol-water mixtures and acetonitrile mixtures[54] in relation to concentration of solvent in mole fraction

was investigated using global analysis procedure. Again, this solution was indeed found to be single exponential under ambient conditions and this was independent of emission wavelength. Global analysis gave a lifetime of $2.2 \mathrm{~ns}\left(\chi^{2}=1.13\right)$ for the methanol-water solution. The quality of the fitted data is evident in Figure 3.10, which shows data, fit and residuals for the mixture at an emission wavelength of $510 \mathrm{~nm}$.

A number of studies of ANS fluorescence in binary solvent systems have been reported, but these have not included the measurement of a methanol-water mixture. Fluorescent lifetime measurements of ANS in a ethanol-water mixture have been reported, though the exact nature of the time-resolved fluorescence decays is not clear[45]. The most recent study indicated that under ambient conditions the fluorescence decay of ANS in ethanol-water mixtures was single exponential[54]. This is in contradiction to an earlier study stating that the decays were clearly non-exponential[45]. This particular work was carried out using the old fluorescence lifetime method of an electrophotonics streak camera. The technique and equipment described in this thesis is much advanced of this old method with the obvious advantage of much faster pulses and 


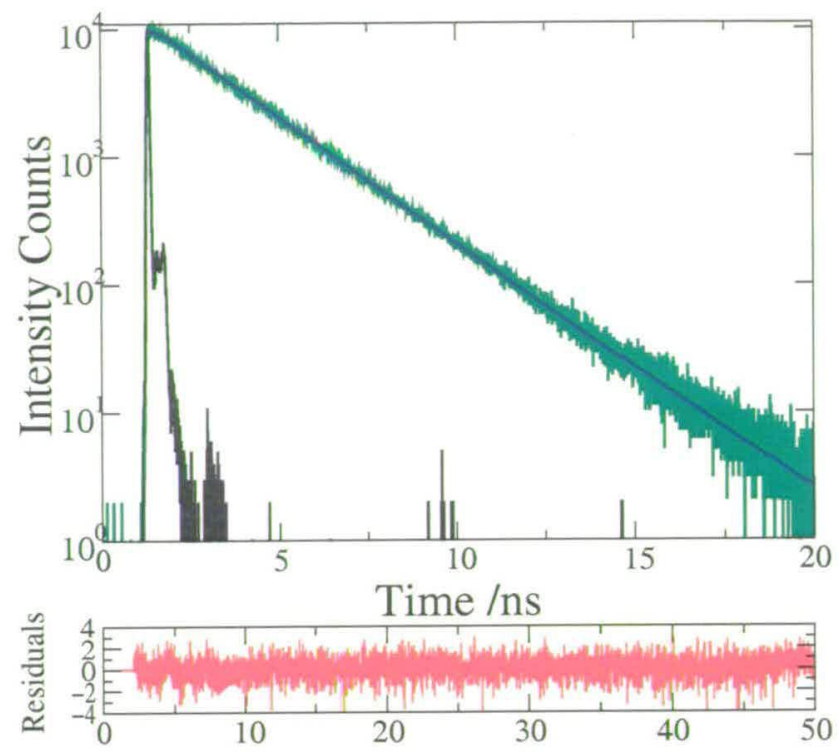

Figure 3.10: The time resolved fluorescence decay of 1,8-anilinonaphthalene sulfonate ANS in methanol-water mixture $x=0.50$ mole fraction at $298 \mathrm{~K}$. The green line shows the fluorescence intensity decay, the black line shows the instrument response function, the blue line shows the exponential fit to the data and the red line shows the residuals, indicating the goodness of fit to the data.

greater accuracy.

To further understand the aqueous alcohol system a large number of experiments were carried out on distinct systems. Firstly, the mixture of ethanol and water was explored. This involved an investigation into the fluorescence lifetime decays of the pure solvent and across the concentration range. This work revealed again that the fluorescence lifetime decay of ANS in ethanol-water solutions could be very accurately described by a single exponential decay. The fluorescence lifetimes obtained were in very good agreement with the previous work of Ebbsen[54]. The higher order alcohols tertiarybutanol and propanol were also explored and a range of concentrations of the aqueous mixtures. Again, the fluorescence lifetime decays could be accurately described by single exponential decays. A number of different fluorescence probes were also used to explore these aqueous systems. The molecules pyrene, dansyl chloride and ethidium bromide were used in the same way as ANS to act as probes for the local solvent environment of methanol-water solutions and ethanol-water solutions. Again, the flu- 


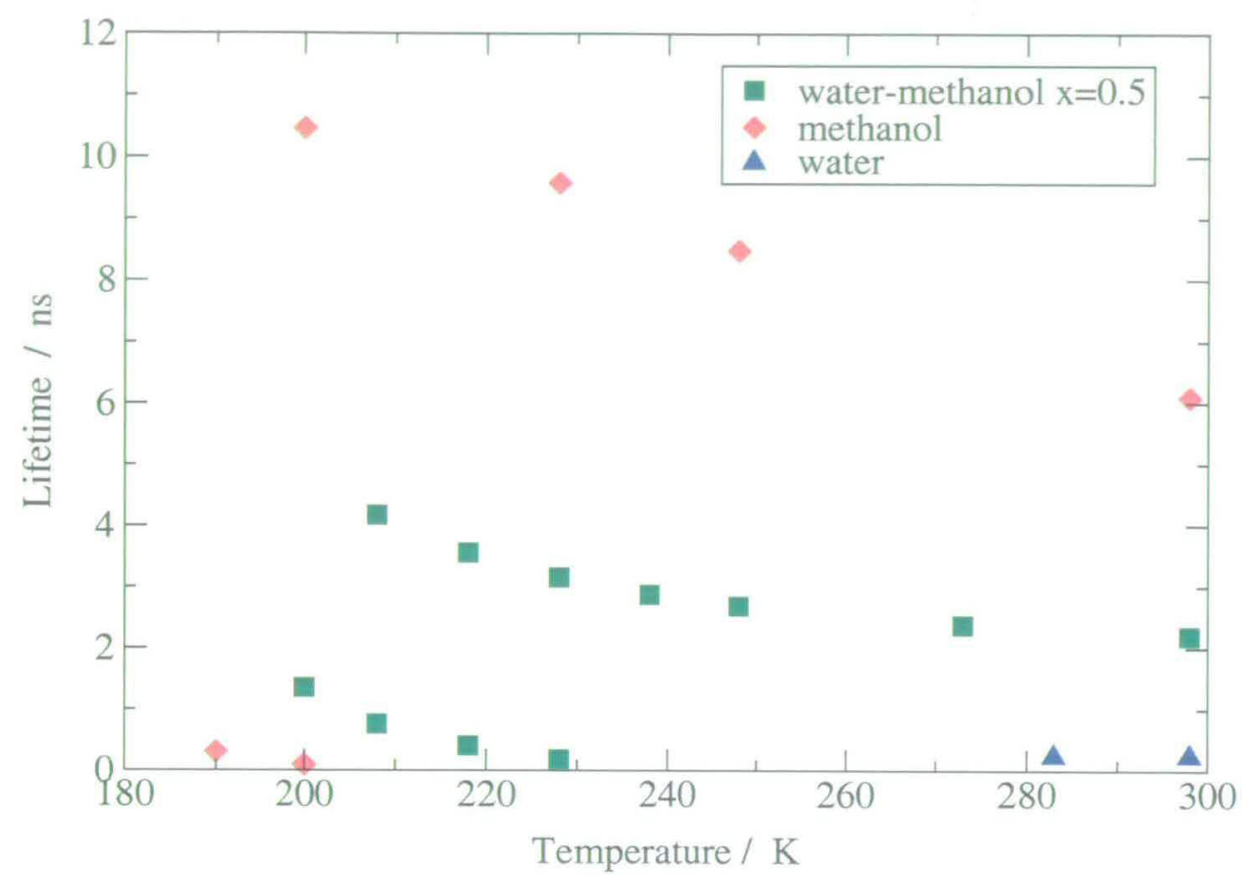

Figure 3.11: Temperature dependence of ANS Fluorescence Decay times in Water $\left(\lambda_{e m}=\right.$ $520 \mathrm{~nm})$, Methanol $\left(\lambda_{e m}=490 \mathrm{~nm}\right)$, or Methanol-Water $x=0.5,\left(\lambda_{e m}=510 \mathrm{~nm}\right)$

orescence lifetime decay could be accurately described by single exponential decay.

Therefore, irrespective of the fluorescence probe used and the alcohol system investigated the fluorescence lifetime decay could be described by single exponential decays. The importance of this result will be discussed further at the end of this chapter.

\subsubsection{Dynamics of the System}

The methanol-water system was investigated under the non-ambient of low temperature to explore the effects on the dynamics of the system. The fluorescence decay was measured for ANS in (i) water, (ii) methanol and (iii) $x=0.50$ mole fraction methanol-water solution as a function of temperature. The results are summarised in Table 3.3 and displayed graphically in Figure 3.11 (where water is represented by $\triangle$, methanol is represented by $\diamond$ and $x=0.5$ mole fraction methanol-water solution is represented by $\mathbf{0})$. 


\begin{tabular}{|c|c|c|c|c|c|c|c|c|}
\hline Solution & $\begin{array}{c}\text { Temp. } \\
\text { /K }\end{array}$ & $\begin{array}{l}\text { Lifetimes } \\
\qquad \tau_{1}\end{array}$ & $\begin{array}{l}\text { (ns) } \\
\tau_{2}\end{array}$ & $\begin{array}{l}\text { Preexp } \\
\qquad \alpha_{1}\end{array}$ & $\begin{array}{l}\text { fact } \\
\alpha_{2}\end{array}$ & $\begin{array}{c}\text { Frac } \\
f_{1}\end{array}$ & $\begin{array}{c}\text { Intens } \\
f_{2}\end{array}$ & $\chi^{2}$ \\
\hline \multirow[t]{2}{*}{ Water } & 298 & 0.241 & & 1 & & 1 & & 1.09 \\
\hline & 283 & 0.242 & & 1 & & 1 & & 1.19 \\
\hline \multirow[t]{5}{*}{ Methanol } & 298 & 6.09 & & 1 & & 1 & & 1.06 \\
\hline & 248 & 8.47 & & 1 & & 1 & & 1.14 \\
\hline & 228 & 9.57 & & 1 & & 1 & & 1.14 \\
\hline & 200 & 10.46 & 0.100 & 0.841 & 0.159 & 0.994 & 0.006 & 1.10 \\
\hline & 190 & 10.75 & 0.310 & 0.817 & 0.183 & 0.998 & 0.002 & 1.07 \\
\hline Meth:Water & 298 & 2.197 & & 1 & & 1 & & 1.08 \\
\hline \multirow[t]{7}{*}{$(x=0.5)$} & 273 & 2.376 & & 1 & & 1 & & 1.09 \\
\hline & 248 & 2.687 & & 1 & & 1 & & 1.05 \\
\hline & 238 & 2.875 & & 1 & & 1 & & 1.06 \\
\hline & 228 & 3.153 & 0.193 & 0.856 & 0.144 & 0.990 & 0.010 & 1.01 \\
\hline & 218 & & 0.416 & 0.831 & 0.169 & 0.977 & 0.023 & 1.02 \\
\hline & 208 & 4.168 & 0.769 & 0.829 & 0.171 & 0.963 & 0.037 & 1.04 \\
\hline & 200 & 4.940 & 1.357 & 0.860 & 0.140 & 0.957 & 0.043 & 1.14 \\
\hline
\end{tabular}

Table 3.3: Fluorescence Decay Parameters of 1,8-anilinonaphthalene sulfonate ANS in Water $\left(\lambda_{e m}=520 \mathrm{~nm}\right)$, Methanol $\left(\lambda_{e m}=490 \mathrm{~nm}\right)$, or Methanol-Water $x=0.5,\left(\lambda_{e m}=510 \mathrm{~nm}\right)$ 
It is evident from Figure 3.11 that the fluorescence decay times increase upon cooling for all three solutions. This is in agreement with a previous study[55], and this commonly observed temperature dependence is attributed to a decrease in the contribution of non-radiative deactivating pathways, $k_{n r}$. From equation 3.4 , this decrease in $k_{n r}$ results in an increase in the fluorescence lifetime $\tau_{f}$.

Let us first consider the effect of cooling on the fluorescence lifetime decay of ANS in methanol ( $\diamond$ on Figure 3.11). The lifetime of ANS in pure methanol is single exponential until a temperature of around $200 \mathrm{~K}$ is reached. At this point the data is best described by bi-exponential kinetics, with a long component of $10.5 \mathrm{~ns}$ and a short component of 100 ps (Table 3.3). On cooling to $193 \mathrm{~K}$ this short component increases to around $300 \mathrm{ps}$.

A global analysis procedure was carried out on this system which, as before, involved measuring fluorescence intensity decays at various wavelengths across the emission spectrum. Table 3.4 shows the results of a global analysis study on an ANS methanol solution at $200 \mathrm{~K}$. The fluorescence decay was measured at 5 emission wavelengths and the decay was fitted with a bi-exponential decay with two fluorescence lifetimes $\tau_{1}$ and $\tau_{2}$. Also shown are $\alpha_{1}$ and $\alpha_{2}$, the pre-exponential factors and $f_{1}$ and $f_{2}$, the fractional intensities. The global analysis wavelength dependent study at $200 \mathrm{~K}$ shows the pre-exponential factor $\alpha_{2}^{b}$ for the short lifetime component varies from a positive decay at short wavelength to a negative rise time at long wavelength (Table 3.4). This variation in the pre-exponent factor or amplitude can be visualised in Figure 3.12.

Next, let us consider the effect of cooling on the fluorescence lifetime decay of the aqueous solution ( symbols). Table 3.4 shows the results of a global analysis study on an ANS methanol-water $x=0.50$ mole fraction solution at $200 \mathrm{~K}$. The fluorescence decay was measured at 5 emission wavelengths and the decay was fitted with a biexponential decay with two fluorescence lifetimes $\tau_{1}$ and $\tau_{2}$. At $298 \mathrm{~K}$ the fluorescence decay of this methanol-water solution could be described by a single exponential decay. 


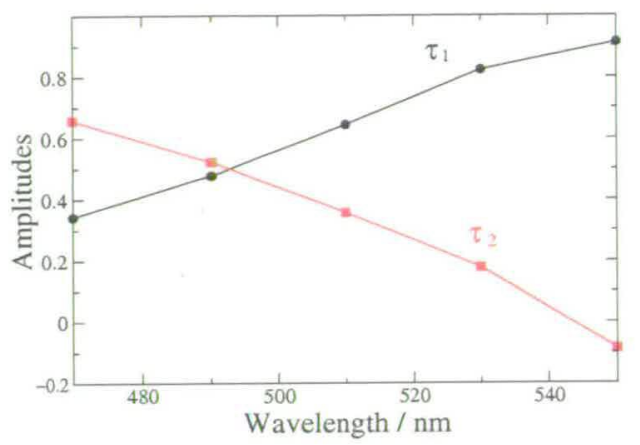

Figure 3.12: Wavelength dependence of the amplitudes of $\tau_{1}$ and $\tau_{2}$ for the bi-exponential fit of the fluorescence decay of ANS in methanol at $200 \mathrm{~K}$

On cooling the fluorescence decay shows a transition to bi-exponential kinetics. Figure 3.13 shows the time resolved fluorescence decay of ANS in $x=0.50$ methanol-water solution at $200 \mathrm{~K}$. The fluorescence decay can be accurately fitted by a experiential decay with $\tau_{f}^{1}=4855$ ps and $\tau_{f}^{2}=943$ ps.

The global analysis wavelength dependent study at $200 \mathrm{~K}$ shows the pre-exponential factor $\alpha_{2}^{b}$ for the short lifetime component varies from a positive decay at short wavelength to a negative rise time at long wavelength (Table 3.4). This variation in the pre-exponent factor or amplitude can be visualised in Figure 3.14. However, in this case the transition from single to pre-exponential kinetics occurs at a much higher temperature $(\sim 230 \mathrm{~K})$ and the difference between the two lifetimes is much less. The shorter lifetime is an order of magnitude greater than for the methanol solution at 200 K.

The onset of bi-exponential kinetics observed for ANS in methanol and ANS in $\mathrm{z}$ $x=0.50$ mole fraction solution is due to a slowing of solvent relaxation dynamics upon cooling. The short component which is observed and its wavelength dependent change in sign is thought to be clear signature of solvent relaxation. This process will now be explained.

According to the standard model[43] solvent molecules around the ground-state fluorophore retain the same orientational arrangement during the electronic transition upon 


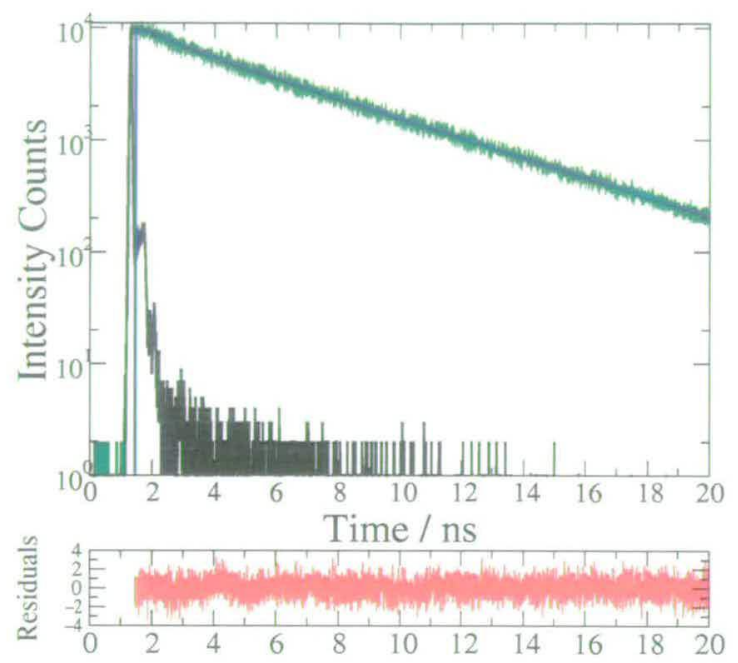

Figure 3.13: The time resolved fluorescence intensity decay of 1,8-anilinonaphthalene sulfonate ANS in methanol-water mixture $x=0.50$ mole fraction at $200 \mathrm{~K}$. The green line shows the fluorescence intensity decay, the black line shows the instrument response function, the blue line shows the exponential fit to the data and the red line shows the residuals, indicating the goodness of fit to the data.

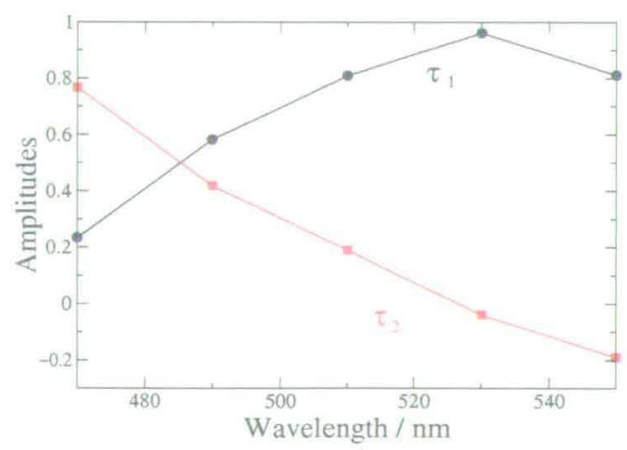

Figure 3.14: Wavelength dependence of the amplitudes of $\tau_{1}$ and $\tau_{2}$ for the bi-exponential fit of the fluorescence decay of ANS in methanol-water $x=0.50$ mole fraction at $200 \mathrm{~K}$ 


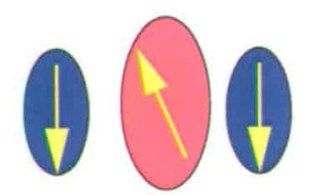

Solvent Dipoles Unaligned

with Excited Fluorophore Dipole

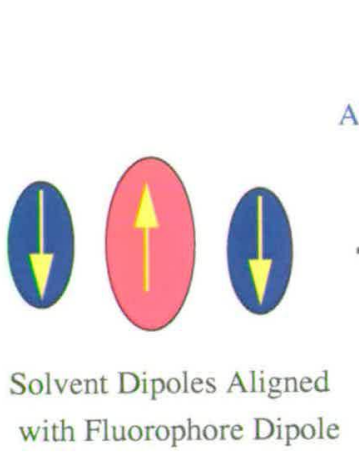

Figure 3.15: A simple schematic representation of solvent relaxation. $S_{0}$ represents the equilibrium ground state of the ANS molecule, $S_{1}^{\prime}$ and $S_{1}^{\prime \prime}$ the non-equilibrium excited states, $S_{1}$ the equilibrium excited state and $S_{0}^{\prime}$ the non-equilibrium ground state. The vertical blue line represents absorption, the vertical green line represents emission and the dashed vertical red line represents solvent relaxation.

excitation. If the dipole moment of the fluorophore in the excited state is different, then solvent molecules re-orient to a new equilibrium orientational distribution. This is known as solvent relaxation[56], [57], [58], [51], [59]. The relaxation of molecules solvating the ANS molecule and can be understood using an illustration of a simplified Jablonski diagram (Figure 3.15).

After absorption of light (represented by the vertical blue line) the ANS molecule shifts from its equilibrium ground state $\left(S_{0}\right.$ in Figure 3.15$)$ to its excited state (non equilibrium Frank-Condon excited state $S_{1}^{\prime \prime}$ ). The dipole moment of the ANS molecule in its excited state is greater than in the ground state[56] and hence the interaction of an excited ANS molecule with surrounding molecules is different from that before absorption. Reorientation and translation of nearest-neighbour molecules allow the ANS molecule to relax gradually to is equilibrium excited state $\left(S_{1}\right)$.

In solutions of low viscosity where these relaxations are very fast the fluorescence 
almost always takes place from the equilibrium excited state $\left(S_{1}\right)$. In other words, practically all the ANS molecules reach the $S_{1}$ state before emission of the fluorescence photon (represented by vertical green line). In this situation $\tau_{R} \ll \tau_{f}$, where $\tau_{R}$ is the relaxation lifetime and $\tau_{f}$ the fluorescence lifetime. On emitting the photon the ANS molecule returns to its non-equilibrium ground state $S_{0}^{\prime}$ which finally relaxes to the equilibrium ground state $S_{0}$ (Figure 3.15).

In more viscous solutions, the relaxation (represented by the red dashed line) of the molecules surrounding ANS may be so slow that the time required for the relaxation to the equilibrium excited state $S_{1}$ is comparable or even greater than the fluorescence lifetime of the excited state. In this situation $\tau_{R} \gg \tau_{f}$ and many ANS molecules will emit fluorescence photons before reaching the equilibrium excited state $S_{1}$. Hence, the emission takes place from one of the energetically higher excited states. One of such intermediate non-equilibrium excited states $S_{1}^{\prime}$ and the corresponding fluorescence is illustrated by the red dashed line in Figure 3.15. As the viscosity of the environment changes, solvent relaxation will either play a bigger role or be less evident, and hence the fluorescence emission maximum in the fluorescence spectrum will change.

In conclusion, experiments have shown the onset of bi-exponential kinetics for ANS in methanol and ANS in z $x=0.50$ mole fraction solution is due to solvent relaxation. The dynamics of the system can be readily understood using a simple two-state solvent relaxation model[60],[61].

\subsection{Discussion}

ANS fluorescence has received considerable attention over the years, owing to extensive use as a biomolecular probe[49], [62]. ANS is an ideal probe of the structure and dynamics of a water-methanol mixture because of the 24-fold difference in lifetime of ANS in water compared with ANS in methanol. Whilst the effect of solvent on flu- 


\begin{tabular}{|c|cccc|cccc|}
\hline & ANS & $x=0.5$ & Methanol & Water & ANS & $x=1.0$ & Methanol & \\
& $\tau_{1}=$ & $4.85 \mathrm{~ns}$ & $\tau_{2}=$ & $0.795 \mathrm{~ns}$ & $\tau_{1}=$ & $9.85 \mathrm{~ns}$ & $\tau_{2}=$ & $0.115 \mathrm{~ns}$ \\
$\lambda_{e m} \mathrm{em}$ & $\alpha_{1}^{b}$ & $f_{1}^{b}$ & $\alpha_{2}^{b}$ & $f_{2}^{b}$ & $\alpha_{1}^{b}$ & $f_{1}^{b}$ & $\alpha_{2}^{b}$ & $f_{2}^{b}$ \\
\hline 470 & 0.233 & 0.650 & 0.767 & 0.350 & 0.343 & 0.978 & 0.657 & 0.022 \\
\hline 490 & 0.582 & 0.894 & 0.418 & 0.106 & 0.478 & 0.987 & 0.522 & 0.013 \\
\hline 510 & 0.809 & 0.963 & 0.191 & 0.037 & 0.644 & 0.994 & 0.356 & 0.006 \\
\hline 530 & 0.961 & 0.993 & -0.039 & -0.007 & 0.823 & 0.997 & 0.177 & 0.003 \\
\hline 550 & 0.811 & 0.963 & -0.189 & -0.037 & 0.913 & 0.999 & -0.087 & -0.001 \\
\hline
\end{tabular}

Table 3.4: Global Analysis of a Two Component Mixture of 1,8-anilinonaphthalene sulfonate(ANS) in $x=0.5$ and $x=1$ Methanol-Water solution at $200 \mathrm{~K}$ Measured at 5 Emission Wavelengths, where $\alpha_{1}$ and $\alpha_{2}$ are pre-exponential factors and $f_{1}$ and $f_{2}$ are fractional intensities.

orophores has been the subject of considerable investigation[43], [63], relatively little of this has focused on mixed solvent systems (e.g. [63]-[64]). In addition, the solvent environment of the fluorescence molecules in biological complex systems is more akin to a mixed solvent than a pure solvent, for example, water and hydrocarbon-like medium near the interface of a bilayer membrane.

A recent notable exception is a study of intra-molecular non-radiative decay $k_{n r}$ of the fluorescence probe rhodamine 3B in ethanol-water mixtures[65]. In this study the full range of ethanol-water mixtures concentrations was explored and the effect of different polarity and viscosity on the radiationless rate $k_{n r}$ of decay was investigated. The trend in variation of composition was seen to reflect the dependence on the viscoelastic response of ethanol-water mixtures and produced evidence of solvent clustering. The clustering of ethanol at low ethanol content was interpreted to disrupt the water structure and induce enhanced hydrophobicity around the chromophore which manifests in non-Markovian effects of the reaction rates, $k_{n r}$. However, the rhodamine 3B probe has essentially identical lifetimes in either of the pure solvents, so the use of ANS and 
its fluorescence lifetime is a very different approach to the investigation of molecular segregation.

In general, studies of fluorophores in mixed solvents have shown complex fluorescence decays, in some cases attributed to a distribution of fluorophore environments [43], [66], but also to site-specific solvated species[67]. In this context, the first very interesting feature of the fluorescence of ANS in the methanol-water mixture is that it displays single exponential kinetics at ambient temperatures (Figure 3.10).

At low temperature, the lifetime of ANS in both methanol and the mixed solvent are best described by bi-exponential kinetics. The wavelength dependent data were globally analysed and were found to fit a two-state solvent relaxation model. It may seem surprising that such a simple kinetic scheme can explain solvent relaxation, which is known to be an extremely complex process[63]. However, it has been demonstrated in a number of independent studies that solvent relaxation in certain systems (notably where charge transfer is involved) is best described by a two-state process [60], [61], [68], [69].

Solvent relaxation can often be correlated with simple properties relating to solvent mobility such as viscosity[68], [70]. For most low viscosity solvents under ambient conditions solvent relaxation typically occurs on a picosecond timescale, resulting in fluorescence being almost exclusively observed from the solvent-relaxed state. This has been demonstrated for ANS in pure water or methanol at room temperature[62]. Since methanol $(\eta=0.0547 \mathrm{cP})$, water $(\eta=0.89 \mathrm{cP})$ and methanol-water $x=0.50$ mole fraction solution $(\eta=1.9 \mathrm{cP})$ have relatively low viscosities[71] at room temperature solvent reorientation is expected to be very fast. The rotational correlation time of a molecule depends on the molecular volume and viscosity $\left(\tau_{\text {rot }}=\eta V / k_{B} T\right.$, where $k_{B}$ is the Boltzmann constant, $\mathrm{T}$ is the temperature, $\eta$ is the viscosity and $\mathrm{V}$ is the molecular volume[43]). If the ANS molecule has a volume of $\sim(3 \AA)^{3}$ then at room temperature the rotational correlation time of ANS will be $\sim 4$ ps in methanol, $\sim 7$ ps 
in water and $\sim 14 \mathrm{ps}$ in methanol-water $x=0.50$ mole fraction at room temperature. These rotational correlation times can be equated to the reorientational relaxation rates of the fluorescence molecule in the solutions. Since these relaxation times are very fast, most of the ANS molecules will have relaxed to their equilibrium excited state before emitting a photon. Therefore, only one fluorescence lifetime will be observed, which is seen experimentally.

However, as the system is cooled the viscosities are increasing. In the methanol-water mixture $x=0.50$ mole fraction at $200 \mathrm{~K}$ the viscosity has increased by a large amount to $\eta=90.4 \mathrm{cP}$. This leads to a new $\tau_{\text {rot }} \sim 650 \mathrm{ps}$. Therefore, on cooling the methanolwater system has become much more viscous. This increased viscosity slows down the reorientational relaxation rates of the fluorescence molecule in the solutions. Consequently, the time required for relaxation to the equilibrium excited state may be so slow that some of the ANS molecules will emit a photon from one of the energetically higher excited states. Therefore, two fluorescence lifetimes will be observed and the two state model of relaxation will become applicable.

It has also recently been proposed that the low-temperature dynamics of aqueous solvents is dominated by the behaviour of the aqueous component[72]. In that study, an extensive series of Brillouin scattering experiments on simple aqueous solutions were explored over a range of temperatures and pressures. For all the solutes studied, ranging from alcohols to salts, freezing was inhibited and access allowed to temperatures far below the normal supercooling limit of water. Clear spectroscopic evidence of viscoelastic behaviour was found at temperatures around $233 \mathrm{~K}$. This work suggests that the low-temperature viscoelastic dynamics of these aqueous solutions is dominated by the behaviour of the aqueous component which was shown to exhibit a pronounced decrease in relaxation time though the temperature range over which it occurs is inaccessible unless freezing is suppressed by the presence of solutes. The solutions then behave as if they are approaching a glass transition at lower temperatures. The change toward glassy dynamics occurs close to the temperature at which solvent relaxation 
becomes prominent in the methanol-water mixture reported herein. Like the Brillouin study, there is a sharp change at this temperature; in this case it is the lifetime and solvent relaxation time that increase. The time-resolved fluorescence measurements therefore support the explanation that a partial demixing of methanol-water mixtures occurs at the molecular level in these systems.

The mounting evidence of self-association by alcohol molecules in aqueous solutions does not explain the simplicity of the fluorescence intensity decays observed in the present study. Indeed, the temperature and wavelength dependence of the data is consistent with a homogeneous solvent system. An earlier report of the use of a fluorescent molecule as a probe of water-alcohol mixtures also reported single exponential decay kinetics[73]. In that study, the ratio of the intensities of vibronic peaks of pyrene fluorescence was used as an indicator of the degree of pyrene accumulation in selfassociating alcohol aggregates. Whilst steady-state fluorescence measurements indicated self-association was occurring, the observation of single exponential lifetimes was interpreted as being due to the alcohol aggregates having a very short lifetime, relative to the timescale of pyrene fluorescence decay. At room temperature the fluorescence lifetime $\tau_{f}$ of pyrene in water $\sim 200$ ns while in alcohol $\sim 340 \mathrm{~ns}$. In the present study, however, the ANS fluorescence decay is approximately 2-3 orders of magnitude faster than for pyrene, which will allow the solvent exchange dynamics in the binary mixture to be probed on a correspondingly shorter timescale. The difference between the lifetime in pure methanol $\left(\tau_{f} \sim 6 \mathrm{~ns}\right)$ and water $\left(\tau_{f} \sim 0.25 \mathrm{~ns}\right)$ is also far greater for the ANS probe. It would be very useful to gain an insight into the timescale or lifetime of the microsegregated clusters of water and methanol in these solutions. In the methanol-water $x=0.50$ mole fraction solution a single exponential decay was observed. Therefore, it is at least known that the upper limit on the timescale of water clusters is $250 \mathrm{ps}$, however, it is probable that the lifetimes are much shorter than this. A recent study of fast diffusion of small hydrophobic species in water illustrates the feasibility of rearrangement dynamics on these timescales due to rapid hydrogen bond 


\section{fluctuations[74].}

The persistence of clustered structures in these methanol-water systems is reflected in the average lifetime of clusters and single molecules. An estimate of these lifetimes has been obtained from analysis of molecular dynamics trajectories[75]. Simulations were performed within the NVT ensemble, utilising previously tested intermolecular potentials for both methanol [76] and water [77] that predict the structure and dynamics of the single component liquids well. The results from this work is particularly interesting in the simulation performed at the $x=0.7$ methanol mole fraction. Isolated individual water molecules in the solution were found to be short-lived and survived, on average, for only 2 ps before being absorbed into a cluster. However, in rare cases, lifetimes of 100 ps are found. A similar result was found for actual clusters in the solution which showed average lifetimes of about $3 \mathrm{ps}$ though there were also persistent clusters surviving for up to $0.5 \mathrm{~ns}$. Methanol hydrogen-bonded clusters were noted to be much reduced in size by the presence of the water and extremely short-lived; most persisting for approximately $1 \mathrm{ps}$ with no methanol hydrogen-bonded structure lasting for more than $40 \mathrm{ps}$. Thus these simulation results suggest that the extended structures characterising the methanol-water system are very dynamic with rapid shedding and reforming of cluster members.

For ANS to function as a probe of molecular-scale heterogeneity, it is important that it is small enough to sample different solvent environments. An ANS molecule has a small volume of $\sim(3 \AA)^{3}[78]$. However, it is also important to consider whether an ANS molecule has the correct dimensions to sample a methanol rich environment and a water rich environment which may have a preferred topology. If the micro-segregated regions (clusters) are spherical-like then it it would be possible for an ANS molecule to reside in a cluster environment. However, the clusters could also be string-like or sheet-like in shape, which would make it very difficult for ANS to be in a cluster. Another possibility for the simple decay kinetics could involve the ANS having a solvent shell that is independent of the surrounding bulk solvent. Although there is evidence of 
specific interactions of water with ANS[54], the gradual change in fluorescence properties observed upon altering solvent composition makes this explanation unlikely[45]. Finally, it may be that the ANS molecule has a preference to reside in the interfacial regions of bulk solvent and clusters, preventing it from sampling distinct environments. Fluorescence spectroscopy has provided an excellent experimental tool to explore the dynamics of the methanol-water system. To learn more about the observed microsegregation it is now necessary to improve our understanding of the structure of this simple system. From the fluorescence spectroscopy investigations many new questions have arisen. Molecular segregation is known to exist in these systems but no information is available on what the clusters look like, their size, their topology and lifetimes. To answer these questions it is necessary to utilise another experimental technique, neutron diffraction. In the next chapter neutron diffraction will be explained in detail. 


\section{Chapter 4}

\section{Neutron Diffraction: Probing the Structure of Liquids}

Neutron diffraction experiments are a very powerful tool for exploring the structure of liquids. These experiments can allow access to real structural information about the methanol-water system under investigation. This chapter gives an introduction to the diffraction experiment and presents the theory of neutron diffraction. A full description of the data correction procedure which must be performed to the experimental data will follow. Finally, computational modelling techniques for extracting useful structural information will be introduced.

\subsection{Neutron Diffraction}

A diffraction experiment allows the investigation of structure over a range of different length scales $(0.1-10 \lambda)$. The basic idea behind scattering of any kind is that the incident radiation used should have a wavelength comparable to the length scale of the system under investigation. When a beam of radiation is incident on a target, the scattered intensity will contain information on the positions of the scattering centres in 
the target.

A neutron is an uncharged subatomic particle with mass 1839 times that of an electron. It has a spin of $\frac{1}{2}$ and a magnetic moment of -1.9132 nuclear magnetons. A neutron with speed $v$ has a de Broglie wavelength given by

$$
\lambda=\frac{h}{m_{n} v},
$$

where $\lambda$ is the wavelength of the neutron, $h$ is Planck's constant, $m_{n}$ is the mass of a neutron and and $v$ is the velocity of the neutron, and thus the neutron exhibits wavelike behaviour including diffraction. Neutrons scatter from materials by interacting with the nucleus of an atom rather than the electron cloud. Neutrons have wavelengths similar to atomic spacings, permitting diffraction measurements to be performed. Neutrons with wavelengths in the range 1-10 $\AA$ are therefore ideal to investigate the structural correlations in aqueous solutions. These wavelengths correspond to energies of the order of meV which can be obtained at most neutron facilities. Experiments involving neutrons are highly advantageous for studying aqueous systems for a number of reasons.

- It is easy to sense light atoms, such as hydrogen, in the presence of heavy atoms.

- Neighbouring elements in the periodic table generally have substantially different scattering cross sections and can be distinguished.

- The interaction of a neutron with the nucleus of an atom is weak (but not negligible) making them a highly penetrating probe.

- The nuclear dependence of scattering allows isotopes of the same element to have substantially different scattering lengths for neutrons. 


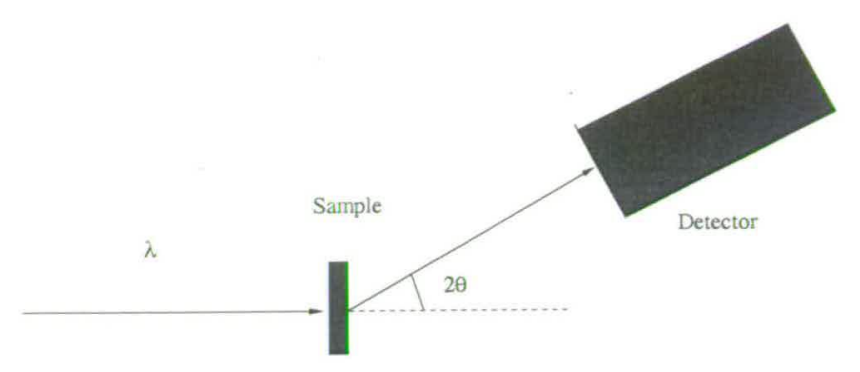

Figure 4.1: The diffraction experiment

\subsubsection{The Diffraction Experiment}

The neutron diffraction experiment can be understood very simply. A thin sample of liquid is set in the path of radiation of given wavelength $\lambda$, where $\lambda$ is of the order of the molecular spacings in the liquid (Figure 4.1). The intensity of the scattered radiation $I(\theta)$ is determined using detectors, and $I(\theta)$ is recorded as a function of $\theta$. This quantity is Fourier transformed to give the familiar function $g(r)$, the radial distribution function which measures the probability of finding a molecule at a distance from a central molecule. This important function will be presented in more detail in the next section.

All the diffraction experiments performed in this thesis were carried out on SANDALS, the Small Angle Neutron Diffractometer for Amorphous And Liquid Samples at the ISIS facility at Rutherford Appleton Laboratory in the UK. ISIS is a pulsed neutron source and uses the time-of-flight measurement. Charged particles (protons) are accelerated in a particle accelerator and then collide with a material (a tantalum target) that produces neutrons from nuclear reactions in a short pulse. The pulse is directed onto the sample and from the sample to a neutron detector, where it is recorded as a function of time:

$$
t=\frac{m_{n}}{h} L \lambda
$$

where $t$ is the time-of flight, $m_{n}$ is the mass of a neutron, $h$ is Planck's constant, $L$ is the 
path length and $\lambda$ is the wavelength of the neutron. As the distance from the source to the sample and sample to the detector is known, neutron velocities and hence energy and wavelength can be measured. The instrument SANDALS offered the scattering angles range of $2 \theta\left(3.5^{\circ}-37^{\circ}\right)$ and neutron wavelengths $[0.05 \AA-4.5 \AA]$.

\subsubsection{Neutron Diffraction Under Non-Ambient Conditions}

The high pressure experiments were also carried out on the SANDALS instrument. The sample pressure was regulated and monitored using a pressure rig. A detailed description of the experimental apparatus used is given in reference[79]. Figure 4.2 shows a cut away view of the interior of the $\mathrm{Ti}-\mathrm{Zr}$ can used to hold the liquid sample. It consisted of an array of seven cylindrical holes (1.5 $\mathrm{mm}$ diameter) drilled into Ti-Zr alloy. This material was used because of its high strength and excellent corrosion resistance to high pressure and temperature aqueous solutions. The alloy has, on average, a zero coherent scattering amplitude for neutrons. This makes Ti-Zr an excellent choice for housing the sample since it makes very little contribution to the total coherent scattering from the sample. The container wall thickness was chosen to allow pressures up to $5 \mathrm{kbar}$ to be handled safely. A schematic view of the pressure handling system is shown in Figure 4.3. Heaters were placed at the top and bottom of the cell and controlled independently. The temperature was kept stable to within $0.2 \mathrm{~K}$ and temperature uniformity was of the order of $0.5 \mathrm{~K}$ from the top and bottom to the middle of the container.

\subsection{From Scattered Intensity to Structure Factor}

It is important to understand how the scattered intensity $I(\theta)$ maps onto the spatial structure of the system under investigation. What follows is an explanation of the theory of scattering in liquid systems. Figure 4.4 illustrates the scattering geometry of 


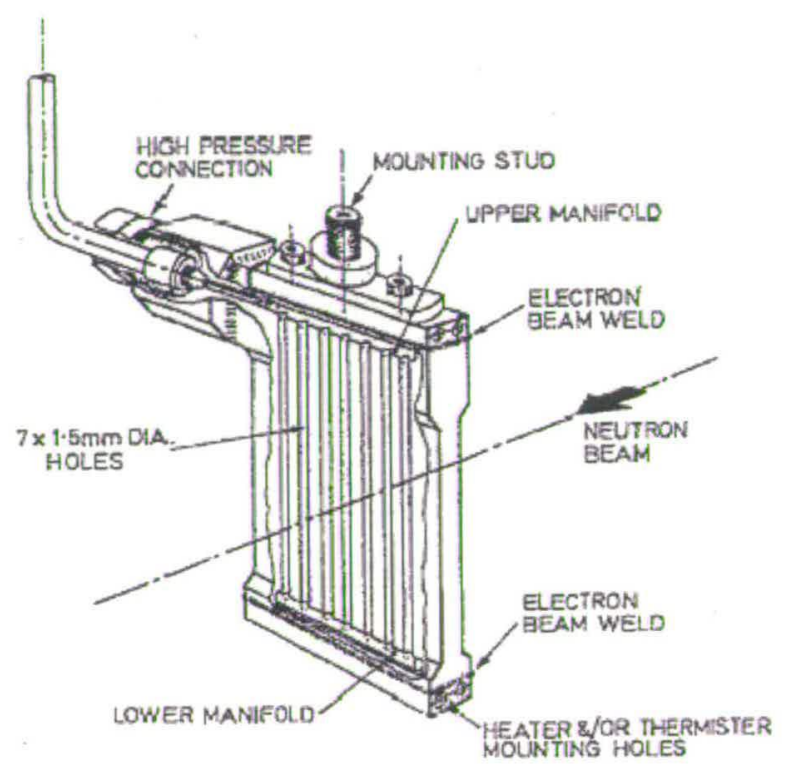

Figure 4.2: Cut away view showing the interior of the Ti-Zr sample can[79]

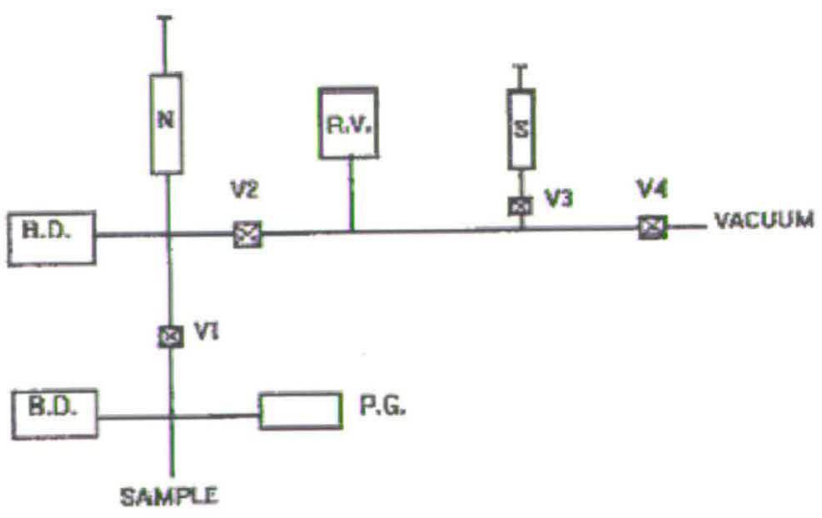

Figure 4.3: Schematic view of the pressure handling system. V1, V2, V3 and V4 are valves, S is a syringe used to fill up the system with water after evacuation, $R V$ is a release valve (100 bar), $\mathrm{N}$ is a pressure generator, $\mathrm{BD}$ are two bursting discs (5000 bar) and $\mathrm{PG}$ is the pressure gauge[79] 


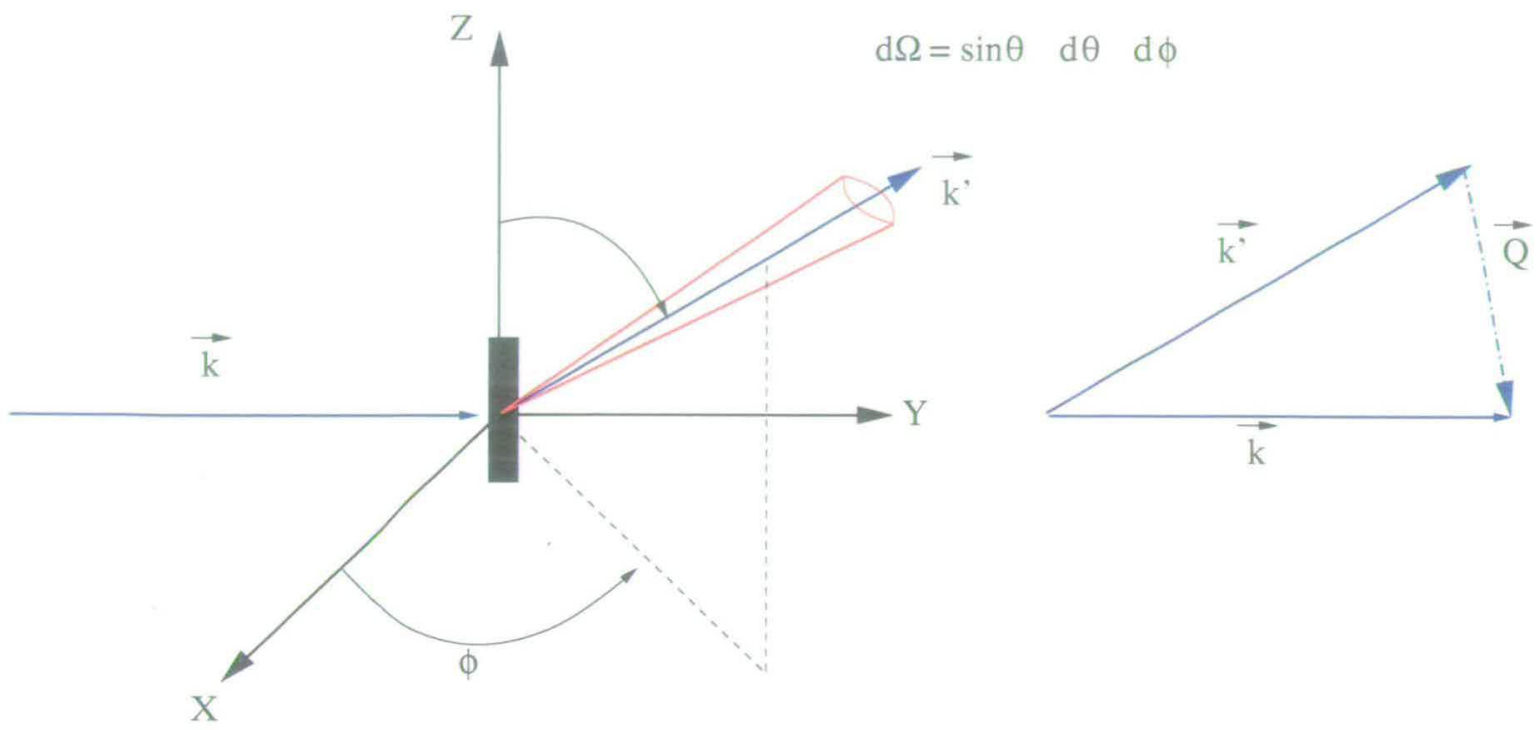

Figure 4.4: Illustration of the geometry of a scattering experiment with incident wave vector $k$ and scattered wave vector $k^{\prime}$. The solid angle $\mathrm{d} \Omega$ is defined about a specific direction $\theta$ and a detector collects particles scattered in the direction defined by the angle $\theta$

a sample in a scattering experiment with incident wave vector $\vec{k}$ and scattered wave vector $\overrightarrow{k^{\prime}}$ (where $k=2 \pi / \lambda$ ). The collision is elastic and so the incoming and outgoing scattering vectors have the same magnitude. The scattering vector $\vec{Q}$ is a measure of the momentum transfer to the sample and is defined as:

$$
\vec{Q}=\vec{k}-\overrightarrow{k^{\prime}}
$$

The solid angle $d \Omega$ is defined about a specific direction $\theta$ and a detector collects particles scattered in the direction defined by the angle $\theta$

If the particle interacts with the scattering medium via a potential $U$, then by Fermi's Golden Rule the scattering amplitude is proportional to the matrix element formed by the incoming wave function of the incident particle, the scattering potential and the outgoing wave function of the scattered particle ${ }^{1}$.

\footnotetext{
${ }^{\mathrm{I}}$ This is only true if the interaction is sufficiently weak that only the lowest order scattering needs to be considered for the entire sample
} 


$$
\text { amplitude } \approx\langle\text { out }|U| \text { in }\rangle
$$

\subsubsection{The Scattering Amplitude}

This scattering amplitude is denoted by $A_{k, k^{\prime}}$ where the two plane wave states of the scattered particle are characterised by the incident and scattered wave vectors. Therefore, we can express this amplitude as:

$$
A_{k, k^{\prime}}=\int e^{-i \vec{k}^{\prime} \cdot \vec{r}} U(\vec{r}) e^{i \vec{k} \cdot \vec{r}} d^{3} \vec{r},
$$

where $e^{-i \vec{k} \vec{r}}$ is the wave function of the incident particle and $e^{-i \vec{k} \cdot \vec{r}}$ is the wave function of the scattered particle.

This is the scattering amplitude for a single particle system. Let us now consider the case of a multi-particle system.

The interaction potential for a multi-particle system $U(\vec{r})$ is given by:

$$
U(\vec{r})=\sum_{\alpha} U_{\alpha}\left(\vec{r}-\vec{r}_{\alpha}\right)
$$

where $r_{\alpha}$ is the position of an atom arbitrarily labelled as $\alpha$. The amplitude of the scattered wave is given by:

$$
A_{k, k^{\prime}}=\sum_{\alpha} \int e^{-i \vec{k}^{\prime} \cdot \vec{r}} U_{\alpha}\left(\vec{r}-\vec{r}_{\alpha}\right) e^{i \vec{k} \cdot \vec{r}} d^{3} \vec{r}
$$

This can be written in a more convenient form by defining a variable $\vec{R}_{\alpha}=\vec{r}-\vec{r}_{\alpha}$ so that the scattering form factor (which will be defined later) appears multiplicatively times a factor with information about atomic positions. 


$$
A_{k, k^{\prime}}=\sum_{\alpha} \int e^{-i \vec{k}^{\prime} \cdot\left[\vec{r}_{\alpha}+\vec{R}_{\alpha}\right]} U_{\alpha}\left(\vec{R}_{\alpha}\right) e^{i \vec{k} \cdot\left[\vec{r}_{\alpha}+\vec{R}_{\alpha}\right]} d \vec{R}_{\alpha}
$$

Grouping the terms in $R_{\alpha}$ together and using equation 4.3 for the scattering momentum we get for the scattering amplitude:

$$
A_{k, k^{\prime}}=\sum_{\alpha}\left[\int e^{i \vec{Q} \cdot \vec{R}_{\alpha}} U_{\alpha}\left(\vec{R}_{\alpha}\right) d \vec{R}_{\alpha}\right] e^{i \vec{Q} \cdot \vec{r}_{\alpha}}
$$

This can be written as:

$$
A_{k, k^{\prime}}=\sum_{\alpha} U_{\alpha}(\vec{Q}) e^{i \vec{Q} \cdot \vec{r}_{\alpha}}
$$

where the integral in equation 4.9 can be denoted by $U_{\alpha}(\vec{Q})$. This term is known as the atomic form factor and contains all the information on the spatial extent of the interaction potential.

\subsubsection{The Scattered Intensity}

The scattered intensity is the square of the matrix element $A_{k, k^{\prime}}$ derived above:

$$
I(\vec{Q})=\left|A_{k, k^{\prime}}\right|^{2}
$$

In a typical scattering experiment, the differential cross section $\frac{d \sigma}{d \Omega}$ is the experimentally accessible quantity. It is defined as the ratio of the number of particles scattered into the direction $(\theta, \sigma)$ per unit time, per unit solid angle $(d \Omega)$ divided by the incident flux [80]. $\frac{d \sigma}{d \Omega}$ is a static cross section, i.e. it is obtained from a scattering experiment by integrating over all possible energy transfers to the medium. It is therefore a function of the scattering vector $\vec{Q}$.

The differential cross section is proportional to the matrix element squared: 


$$
\frac{d \sigma}{d \Omega} \sim \sum_{\alpha, \alpha^{\prime}} U_{\alpha}(\vec{Q}) U_{\alpha}^{*}(\vec{Q}) e^{i \vec{Q} \cdot \vec{r}_{\alpha}} e^{-i \vec{Q} \cdot \vec{r}_{\alpha^{\prime}}}
$$

This equation expresses the scattering matrix element $A_{k, k^{\prime}}$ for a particular configuration, specified by the position vectors $r_{\alpha}$ of atoms in the sample. Figure 4.4 shows a typical scattering geometry illustrating the incident wave vector $\vec{k}$, scattered wave vector $\overrightarrow{k^{\prime}}$ and scattering vector $\vec{Q}$. If the positions of the atoms in the sample in Figure 4.4 are rigidly fixed, as they would be classically at absolute zero, then equation 4.12 gives the correct differential cross-section. However, in real materials, particles move about, probing large regions of phase space and some ensemble average of the ideal cross-section is required. If the detector accepts all particles scattered by a certain wave vector independent of energy change, then each scattering event takes a snapshot of the sample. In a real experiment, data is collected over a period of time that is very long compared to thermal equilibration times. Therefore, the different snapshots correspond to a time average over many sample configurations. Assuming that time averages and averages over all allowed configurations (ensemble averages denoted by the angular brackets) are equivalent ${ }^{2}$ we have the static or quasi-static limit. When the medium under investigation is a homogeneous isotropic fluid, the structural information does not depend on the direction of $\vec{Q}$ but only on its magnitude.

If the atoms in the system are identical, the atomic form factor $U_{\alpha}(\vec{Q})$ in equation 4.12 comes outside the sum and the differential cross section for the scattering from the system becomes:

$$
\frac{d \sigma}{d \Omega} \sim\left|U_{\alpha}(Q)\right|^{2} I(Q)
$$

The scattered intensity can then be defined as:

\footnotetext{
${ }^{2}$ i.e. the system is ergodic
} 


$$
I(\vec{Q})=\left\langle\sum_{\alpha, \alpha^{\prime}} e^{\left.i \vec{Q} \cdot\left[\vec{r}_{\alpha}-\vec{r}_{\alpha^{\prime}}\right]\right\rangle}\right.
$$

and depends only on the positions of the atoms in the scattering medium and not on the nature of the interactions between atoms and the scattering probes.

\subsubsection{The Structure Factor}

For the final step we now need to relate the scattered intensity to the structure factor $S(Q)$ which contains all the relevant information about the structural correlations in the system. The structure factor $S(Q)$ is defined as:

$$
S(\vec{Q})=N^{-1}[I(\vec{Q})]
$$

where $N$ is the total number of particles in the scattering medium. A typical scattering experiment collects scattered particles over a significant $\vec{Q}$ range. When $|\vec{Q}|=0$, $S(\vec{Q})=N$, which corresponds to a straight through beam. For all remaining values of $\vec{Q}$ the structure factor simplifies to:

$$
\begin{gathered}
S(\vec{Q})=\frac{1}{N}\left\langle\sum_{\alpha, \alpha^{\prime}} e^{i \vec{Q} \cdot\left[\vec{r}_{\alpha}-\vec{r}_{\alpha^{\prime}}\right]}\right\rangle, \\
S(\vec{Q})=\sum_{\alpha \neq \alpha^{\prime}} \int e^{i \vec{Q} \cdot \vec{r}}\left\langle\frac{1}{N} \delta\left(\vec{r}-\left[\vec{r}_{\alpha}-\vec{r}_{\alpha^{\prime}}\right]\right)\right\rangle d \vec{r}+1,
\end{gathered}
$$

where the 1 comes from the $\alpha=\alpha^{\prime}$ terms and the term in angular brackets is defined as the pair correlation function $g(\vec{r})$ multiplied by the scattering medium density $\rho$. We can rewrite part of equation 4.17 as

$$
\rho g(\vec{r})=\left\langle\frac{1}{N} \delta\left(\vec{r}-\left[\vec{r}_{\alpha}-\vec{r}_{\alpha^{\prime}}\right]\right)\right\rangle,
$$


and therefore, the structure factor can now be written as:

$$
S(\vec{Q})=1+\rho \int[g(\vec{r})-1] e^{i \vec{Q} \cdot \vec{r}} d \vec{r} .
$$

In this chapter we have seen that the differential cross-section for scattering of plane wave states provides direct information about the spatial structure of many particle systems. The structure factor is an extremely useful quantity, but its structural information is entirely in $Q$ space. For the experimenter, the quantity of interest is $g(\vec{r})$ which gives information about structure in real space. When the medium under investigation is a homogeneous isotropic fluid, the pair correlation function in equation 4.19 does not depend on the direction of $\vec{r}$ but only on its magnitude. The resulting function is the radial distribution function $g(r)$, introduced in Chapter 2, and is the probability of finding an atom a distance $r$ away from any other.

\subsection{Real Liquid Systems}

Let us now think of the differential cross section in terms of another parameter. The scattering length of a nucleus defines the amplitude of the scattered wave. Consider again equation 4.13 where the interaction potential $U(\vec{Q})$ is now substituted by the scattering length $b_{\alpha}$ :

$$
\frac{d \sigma}{d \Omega} \sim\left|\sum_{\alpha} b_{\alpha} e^{i \vec{Q} \cdot \vec{r}_{\alpha}}\right|^{2},
$$

where the scattering length is related to the interaction potential by:

$$
U(\vec{Q})=\frac{2 \pi \hbar^{2}}{m} b .
$$

This can now be simplified[81] to yield: 


$$
\frac{d \sigma}{d \Omega} \sim N\left\langle b_{\alpha}\right\rangle^{2}\left|e^{i \vec{Q} \cdot \vec{r}_{\alpha}}\right|^{2}+N\left(\left\langle b_{\alpha}^{2}\right\rangle-\left\langle b_{\alpha}\right\rangle\right)^{2}
$$

where

$$
\left|\sum_{\alpha} b_{\alpha}\right|^{2}=N\left\langle b_{\alpha}\right\rangle^{2}+N\left(\left\langle b_{\alpha}^{2}\right\rangle-\left\langle b_{\alpha}\right\rangle\right)^{2}
$$

The scattered intensity consists of contributions from two separate differential cross sections.

\subsubsection{Coherent and Incoherent Scattering}

The first term in the equation yields contributions from coherent scattering, which is the quantity of interest.

$$
N\left\langle b_{\alpha}\right\rangle^{2}\left|e^{i \vec{Q} \cdot \vec{r}_{\alpha}}\right|^{2}
$$

It contains all the information on the positions of the nuclei. The phase factor $\left(e^{i \vec{Q} \cdot \vec{r}_{\alpha}}\right)$ considers all nuclei as the same even though individual nuclei can have a range of scattering lengths. It is the coherent contribution to the differential cross section which contains information about the interference information relating to the positions of the atoms.

The second term:

$$
N\left(\left\langle b_{\alpha}^{2}\right\rangle-\left\langle b_{\alpha}\right\rangle\right)^{2}
$$

does not contain a phase term and measures the mean square deviation of each scattering length from the mean. This term is called the incoherent scattering since it contains no positional information. It is important for contributions from incoherent scattering 
to be removed from the measured raw data before analysis can be carried out. This procedure will be described in a later section.

In complex systems such as molecular liquids there are several atomic species and the scattering length $b_{\alpha}$ of each has to be accounted for separately. The atomic form factor $U_{\alpha}$ contains contributions from $b_{\alpha}$ for each of the different atomic species. The total scattered intensity is a sum of several structure factors with appropriate weights proportional to the product of $b$ and the atomic weight fraction $c_{\alpha}$ of each species. For a complex scattering system which is made up of a molecular liquid, the total structure factor measured by a diffraction experiment is a combination of two terms, a "self" term and a "distinct" term.

$$
F(Q)=\sum_{\alpha} c_{\alpha} b_{\alpha}^{2}\left[S_{\alpha}(Q)\right]+\sum_{\alpha \neq \beta} c_{\alpha} c_{\beta} b_{\alpha} b_{\beta}\left[S_{\alpha \beta}(Q)-1\right]
$$

$S_{\alpha \beta}$ is the partial structure factor for atom types $\alpha$ and $\beta$ and is defined as:

$$
S_{\alpha \beta}(Q)-1=4 \pi \rho \int r^{2}\left[g_{\alpha \beta}(r)-1\right] \frac{\sin (Q r)}{Q r} d r
$$

where $\rho$ is the average number density of the system under investigation and $g_{\alpha \beta}(r)$ is the partial pair correlation function for the two atom types.

The "distinct" scattering arises from interference between two distinctly different atoms, whilst the "self" scattering arises from correlations between an atom and itself. The "self" terms contains contributions from incoherent scattering. The "distinct" terms provides information on coherent scattering and is the important quantity that the experimenter is interested in. 


\subsubsection{Inelastic Scattering Considerations}

In a scattering experiment it is also important to consider the effects of inelastic scattering. In most neutron scattering processes the scattering nucleus recoils under neutron impact. The neutron exchanges energy with the scattering system and inelasticity effects have to be accounted for. The structure factor can no longer be considered to be a function of the scattering vector $Q$ alone but is also a function of energy $w$. The resulting structure factor is then a function of $Q$ and $w[82]$. This $S(Q, w)$ is called the dynamic structure factor with respect to $w$ along a path of constant. Hence, the scattering experiment actually integrates $S(Q, w)$ over all energy transfers and gets an ensembled averaged snapshot of the system at $t=0$.

Inelastic scattering takes place particularly in the presence of light atoms such as hydrogen since the mass of hydrogen is comparable to that of a neutron. Inelastic scattering affects the structure factors measured and has to be taken into account when studying systems which contain light atoms. Inelastic scattering effects can be reduced in two ways. Firstly, by working with high energy neutrons and secondly, by working with low scattering angles making pulsed neutron sources the experimental system of choice for studying these systems. A pulsed neutron source produces very high energy neutrons and also provides a wide range of scattering angles to explore different systems. The inelasticity correction is much more severe at high scattering angles than it is at low scattering angle. This means that the instrument SANDALS, which has its detectors placed at low scattering angles, has been very successful in studying light disordered systems, such as aqueous solutions.

The actual raw data collected during a neutron diffraction experiment needs to undergo a number of corrections before meaningful information can be extracted. This data correction procedure will be outlined in the next section. 


\subsection{Isotope Substitution Explained}

Isotope substitution neutron diffraction is one of the most powerful experimental techniques that can directly probe intermolecular structure in the liquid state. In the particular system of interest for this thesis; methanol and water, the liquids both contain similar species. Methanol and water both contain hydrogen and oxygen. To learn more about the structure of this mixture it is vital that species of each liquid can be distinguished. In this way it would be possible to map and differentiate between the behaviour of hydrogens belonging to the methanol methyl group, hydrogens on the methanol hydroxyl group and hydrogens belonging to the water, for example. If this were not possible then the technique of neutron diffraction would fail to give any useful information to the experimenter. For this reason isotope substitution is a vital tool for exploiting the neutron diffraction experiment when studying aqueous solutions.

Isotope Substitution exploits the difference in scattering length between isotopes of the same element.[83] Since scattering of neutrons depends on the scattering length, an experiment can be performed on chemically similar systems which have different isotopes. The scattering intensity is then different for each sample and depends on the response of the neutrons to the isotopes. This technique allows us to pin down the vantage point of the substituted atom in the liquid and survey the liquid environment about that point.

Table 4.1 shows the scattering lengths $b$ and coherent $\left(d \sigma_{c o h}\right)$ and incoherent $\left(d \sigma_{i n c}\right)$ scattering cross-sections for a number of different elements. In particular, it is clear that for hydrogen and its isotope deuterium the scattering lengths are very different.

In a single neutron diffraction experiment, the quantity obtained after the data has been corrected is the total structure factor $F(Q)$. This is defined as:

$$
F(Q)=\sum_{\alpha \beta} c_{\alpha} c_{\beta} b_{\alpha} b_{\beta}\left[S_{\alpha \beta}(Q)-1\right]
$$




\begin{tabular}{|c|c|c|c|}
\hline Element & $\begin{array}{c}\text { Scattering length } \\
\left(10^{-15} \mathrm{~m}\right)\end{array}$ & $\begin{array}{c}d \sigma_{\text {coh }} \\
\left(10^{-28} \mathrm{~m}^{2}\right)\end{array}$ & $\begin{array}{c}d \sigma_{\text {inc }} \\
\left(10^{-28} \mathrm{~m}^{2}\right)\end{array}$ \\
\hline Hydrogen (H) & -3.740 & 1.758 & 79.7 \\
Deuterium (D) & 6.674 & 5.597 & 2.0 \\
Carbon (C) & 6.648 & 5.6 & $<0.02$ \\
Oxygen (O) & 5.830 & 4.23 & $<0.02$ \\
\hline
\end{tabular}

Table 4.1: Scattering lengths and coherent $\left(d \sigma_{c o h}\right)$ and incoherent $\left(d \sigma_{i n c}\right)$ scattering crosssections for hydrogen, deuterium, carbon and oxygen[81]

where $c_{\alpha}$ and $c_{\beta}$ are the concentrations of atoms of species $\alpha$ and $\beta$ respectively, whilst $b_{\alpha}$ and $b_{\beta}$ are their coherent neutron scattering lengths. The summation is over all atom pairs in the sample and $S_{\alpha \beta}(Q)$ are the partial structure factors which result from inter-atomic correlations between atom pairs $\alpha$ and $\beta$.

These partial structure factors are related to the partial pair distribution functions $g_{\alpha \beta}(r)$ by the mathematical relation

$$
S_{\alpha \beta}(Q)-1=4 \pi \rho \int r^{2}\left[g_{\alpha \beta}(r)-1\right] \frac{\sin (Q r)}{Q r} d r
$$

where $\rho$ is the average atomic number density of the sample.

Through the use of isotopes of differing coherent neutron scattering lengths, it is then in principle possible to extract some of the partial structure factors from the total structure factor measured by any single diffraction experiment. Provided that the structure of an isotopically enhanced sample is to first order identical, this is a reliable technique.

\subsubsection{Isotope Substitution Example}

Now, let us consider the system of interest in this thesis, methanol and water. For this relatively complex system performing the much larger number of isotopically dis- 
tinct diffraction experiments that would be necessary to extract all the partial structure factors would be a very tall order. In a methanol-water mixture there are 6 chemically distinct atoms in the system (methyl carbon $\mathbf{C}$, methyl hydrogen $\mathbf{M}$, methanol hydroxyl oxygen $\mathbf{O}$, methanol hydroxyl hydrogen $\mathbf{H}$, water oxygen $O_{W}$ and water hydrogen $H_{W}$. We would require $6 \times 7 / 2=21$ isotopically distinct experiments to yield the 21 partial structure factors. This would be impossible for two main reasons. Firstly, it would be impossible to prepare the 21 distinct solutions as not all elements have isotopes with significantly different scattering lengths and secondly, the requirements for neutron beam time would be extremely high.

There are other ways to get around this problem and obtain all the structural information we require, but the 'best' kind of isotopic substitution we can do is to extract the three intermolecular structural correlations that determine the liquid properties. These three correlations are those between solute molecules, those between solvent molecules and finally between solute and solvent molecules.

Isotope substitutions on the methyl hydrogens can provide information on solutesolute interactions. The methyl hydrogens can provide a unique handle on the molecular correlations. Three samples are needed and scattering data from each is required. The 3 samples are $\mathrm{CH}_{3} O D$ in $D_{2} O, C D_{3} O D$ in $D_{2} O$ and a $1: 1$ mixture of $\mathrm{CH}_{3} \mathrm{OD} / \mathrm{CD} \mathrm{D}_{3} \mathrm{OD}$ in $\mathrm{D}_{2} \mathrm{O}$. Heavy water was used because the information on the structural correlations between methyl groups is required and deuterium has a significantly lower incoherent cross-section compared to hydrogen. Therefore inelastic corrections are minimised.

The total structure factor is then:

$$
F(Q)=c_{X}^{2} b_{X}^{2}\left[S_{X X}(Q)-1\right]+2 c_{X} c_{H} b_{X} b_{H}\left[S_{X H}(Q)-1\right]+c_{H}^{2} b_{H}^{2}\left[S_{H H}(Q)-1\right]
$$

where again $\mathrm{X}$ represents an unsubstituted atom such as carbon, oxygen and hydroxyl 
hydrogens and $\mathrm{H}$ represents substituted atoms, the methyl hydrogens. $S_{X X}(\mathrm{Q})-1$ and $S_{X H}(\mathrm{Q})-1$ are composite partial structure factors (CPSF) and are a neutron weighted average of several partial structure factors.

A similar set of experiments can be performed on the hydroxyl hydrogens of methanol or water or both together. Altogether this yields 9 CPSF. However, a huge amount of information remains hidden in the CPSF. Alternative data modelling techniques are crucial to get the detailed information on partial radial distribution functions and intermolecular orientations which remain hidden in the CPSF. These techniques will be explored at the end of this Chapter.

Therefore, the best kind of substitution which can be done in a methanol-water system is to deuterate the hydrogens on (a) the methyl groups of the alcohol headgroup and (b) the hydroxyl group of the alcohol and (c) the water. For each substitution we work with (a) a fully deuterated mixture; (b) fully hydrogenated mixture; (c) a 50:50 hydrogenated/deuterated mixture. This gives three values of the neutron scattering length of the substituted sites, and hence, for each triplet of substitutions, enables us to obtain three sets of partial structure factors. We call these $S_{H H}(Q), S_{H X}(Q)$ and $S_{X X}(Q)$.

The corresponding set of experiments can now be specified as follows:

1. Solvent-solvent distribution functions are probed through substitution on the water hydrogen sites to yield the composite partial structure factors $S_{H H}(Q)$, $S_{H X}(Q)$ and $S_{X X}(Q)$ for the solvent-solvent correlations. The three experiments are made with (a) $C D_{3} O D$ in $D_{2} \mathrm{O}$, (b) $C D_{3} O H$ in $\mathrm{H}_{2} \mathrm{O}$ and (c)50:50 $\mathrm{CD}_{3} \mathrm{OH} / \mathrm{CD} \mathrm{D}_{3} \mathrm{OD}$ in $\mathrm{H}_{2} \mathrm{O} / \mathrm{D}_{2} \mathrm{O}$

2. Solute-solute distribution functions are probed through substitution on the alcohol methyl hydrogen sites to yield the composite partial structure factors $S_{H H}(Q)$, $S_{H X}(Q)$ and $S_{X X}(Q)$ for the solute-solute correlations. The three experiments 
are made with (a) $C D_{3} O D$ in $D_{2} O$, (b) $\mathrm{CH}_{3} O D$ in $\mathrm{D}_{2} \mathrm{O}$ and (c) 50:50 CD $\mathrm{D}_{3} \mathrm{OD}$ / $\mathrm{CH}_{3} \mathrm{OD}$ in $\mathrm{D}_{2} \mathrm{O}$

3. Solute-solvent distribution functions are probed through substitution on the alcohol hydroxyl hydrogen sites and water hydrogen sites to yield the composite partial structure factors $S_{H H}(Q), S_{H X}(Q)$ and $S_{X X}(Q)$ for the solute-solvent correlations. The three experiments are made with (a) $\mathrm{CD}_{3} \mathrm{OD}$ in $\mathrm{D}_{2} \mathrm{O}$, (b) $\mathrm{CH}_{3} \mathrm{OH}$ in $\mathrm{H}_{2} \mathrm{O}$ and (c)50:50 $\mathrm{CD}_{3} \mathrm{OD} / \mathrm{CH}_{3} \mathrm{OH}$ in $\mathrm{H}_{2} \mathrm{O} / \mathrm{D}_{2} \mathrm{O}$

Thus there appears to be nine isotopically distinct experiments. However, note that samples 1(a), 2(a) and 3(a) are identical. Therefore, only need to perform 7 experiments to yield the 9 composite partial structure factors specified above, namely $S_{H H}(Q), S_{H X}(Q)$ and $S_{X X}(Q)$ for each of the solvent-solvent, solute-solute and solute-solvent cases considered.

Despite the fact that we have only performed 7 different experiments, it is possible to use this data, together with known chemical information, to extract the 21 partial structure factors we require. This is done using a computational modelling method called the Empirical Potential Structural Refinement which will be described in detail. First, the correction which must be performed on the experimental data will be outlined.

\subsection{Correcting the Data}

In the neutron diffraction experiment the liquid sample is contained in a can made from an alloy of Titanium(68\%) and Zirconium(32\%) (Figure 4.5). This alloy has zero coherent scattering. This means that no Bragg peaks are observed in the scattered intensity due to the can.

In a neutron diffraction experiment four sets of data are collected:

1. Background intensity $B$ 


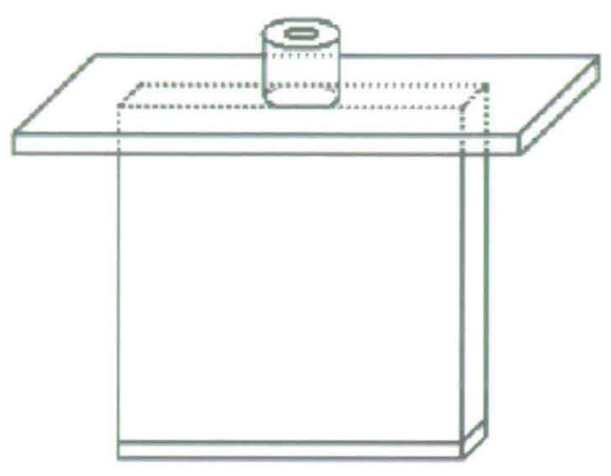

Figure 4.5: Schematic of a flat plate Titanium-Zirconium sample can

2. Neutrons scattered from vanadium $\mathrm{V}$

3. Neutrons scattered from Titanium Zirconium can C

4. Neutrons scattered from Titanium Zirconium can plus sample S+C

In practise the differential cross-section for the sample is determined from performing the following operation with the measured time-of-flight data:

$$
\text { CorrectedResult }=\frac{\text { sample }- \text { container }}{\text { vanadium }- \text { background }}
$$

A fully corrected dataset will also have been corrected for such effects as attenuation, multiple scattering and detector dead-time. An in depth report of these standard data correction procedures are beyond the scope of this thesis. More detailed information on all aspects of these correction procedures can be found in literature references[84], [85]. However, it is important to outline the main features of this data correction procedure. An outline of the steps involved in extracting the structure factor from the raw data will now follow:

- The scattered intensity or detector counts, is collected as a function of scattering angle $\theta$. The 1270 detectors on SANDALS are in approximately cylindrical arrangement in rows, shown in Figure 4.6. The raw data files are amalgamated 


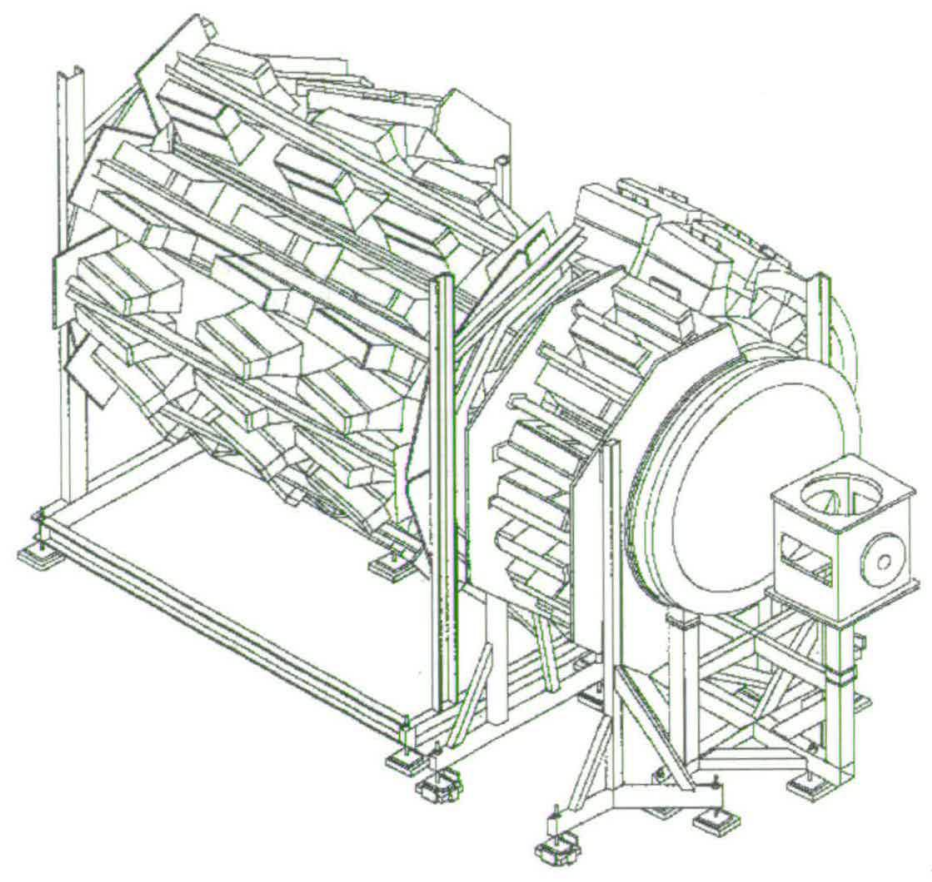

Figure 4.6: Schematic of the detectors on the SANDALS instrument at the ISIS facility, Rutherford Appleton Laboratory, UK

into 18 groups, and each group is specified by a particular scattering angle $\theta$ at a series of angles between $3.5^{\circ}$ and $37^{\circ}$. Before this amalgamation procedure, noisy detectors are eliminated.

- For each detector group, the summed intensity is calculated as a function of scattering vector $\vec{Q}$. The distance travelled by a neutron is calculated from the time-of-flight equation 4.2 in order to calculate the value of $\vec{Q}$ for a given scattering angle. At this stage the incident and transmission spectra are also determined as a function of $\vec{Q}$

- The transmission spectrum is used to determine contributions from multiple scattering events which arise due to; a neutron being scattered by more than one nucleus, and attenuation which occurs due to absorption of some neutrons by the sample. This is determined for the sample + can, the can and vanadium standard data. 
- Intensity measurements from vanadium are also corrected for noise and normalised to the number of scattering nuclei in the vanadium sample.

- Contributions from the can are subtracted from the intensity measurements from the sample + can and the background counts are subtracted from the vanadium data. The vanadium measurement allows the experimenter to normalise out the flux distribution from the moderator. The scattering from vanadium is almost completely incoherent and the Bragg peaks are extremely small.

- The last normalisation involves dividing the entire intensity spectrum by the number of scattering nuclei in the sample itself. The result is a scattering intensity which is a measure of the structure factor and is normalised to the vanadium data to get absolute intensity scales.

- The final step involves removal of the self scattering terms. Procedures to remove these terms can be found in literature[84] and will not be elaborated upon here.

A simple flow chart (Figure 4.7) illustrates all the steps that have been outlined above. This flowchart describes each of the steps involved in a typical data correction procedure for neutron diffraction experiments performed on SANDALS.

\subsection{Computational Modelling Technique}

The Empirical Potential Structural Refinement (EPSR) is a computational method for building atomic and molecular structural models of disordered materials, such as liquids and glasses, that are consistent with available structural data and known physical and chemical constraints.

There are three main ingredients for EPSR to operate:

- Experimental data 


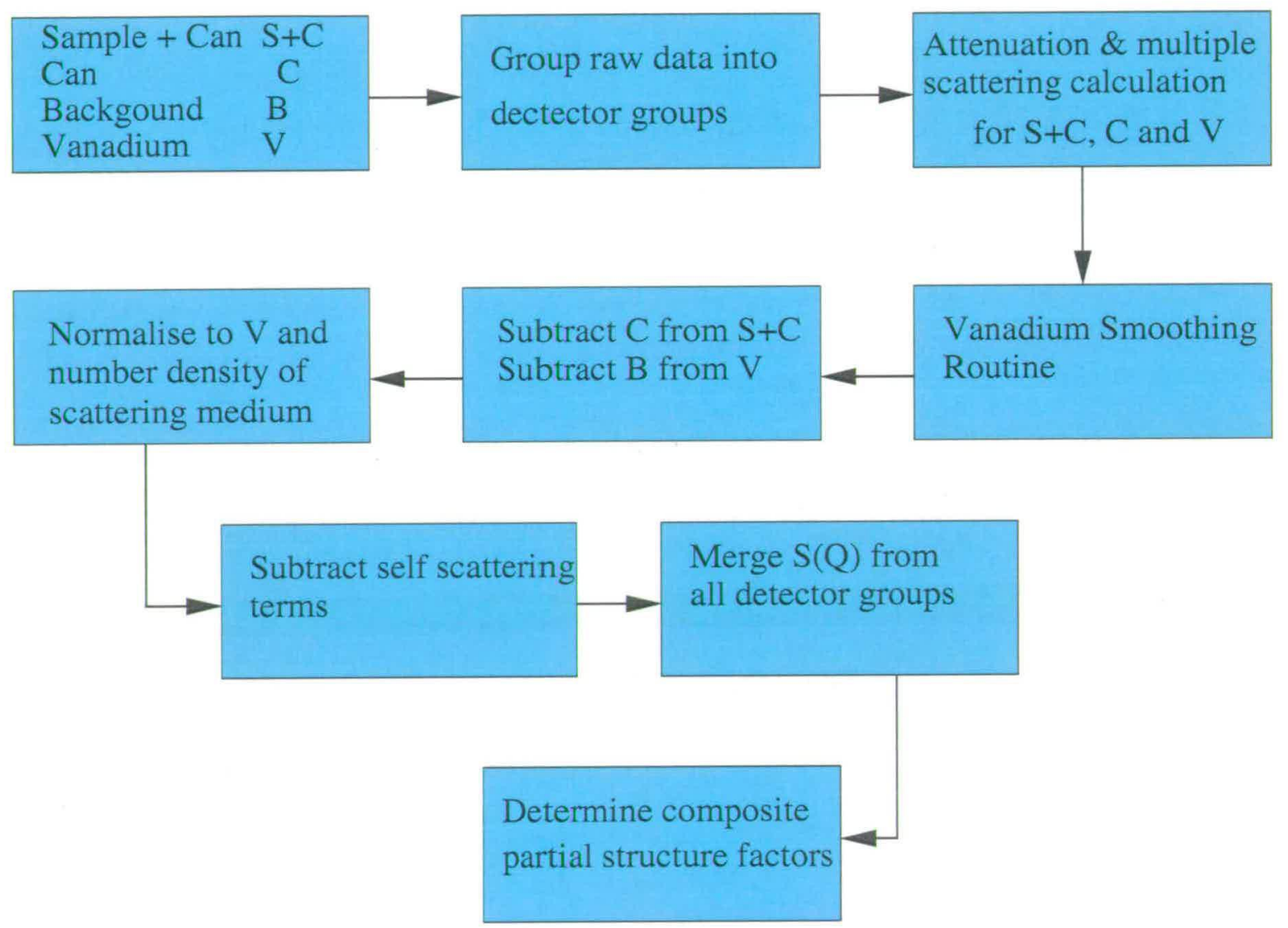

Figure 4.7: A flowchart illustrating the steps involved in the data correction procedure for a neutron diffraction experiment on the SANDALS instrument 
- Monte Carlo computer simulation

- Known constraints such as density and molecular structures.

The experimental data in this case, is the neutron diffraction data obtained on the SANDALS instrument at the Rutherford Appleton Laboratories. The fundamentals of the computer simulation will be explained in the next section.

\subsubsection{Fundamentals of EPSR}

EPSR is a refined Monte Carlo simulation. A number of key items are needed to carry out a Monte Carlo simulation.

- A computer representation of a box of atoms and molecules which stores the coordinates for the system under investigation.

- A set of potential energy functions to model the interactions between atoms in the box.

- A set of rules by which the atoms, molecules and functional groups within the model are moved.

- A set of tools to interrogate the development of the interatomic and intermolecular structures that the model will produce.

There are four types of atom move within EPSR, whole molecule translations, whole molecule rotations, rotation of specific molecular headgroups and individual atomic moves within molecules. A move consists of a small random change in the $(x, y, z)$ coordinates of the atom or molecule, or else a rotation of the molecule by a random amount about a randomly chosen axis.

The acceptance of the move is based on the Metropolis condition[86] which relates to the change in the potential energy of the system as a result of the move. 


$$
\begin{gathered}
\Delta U=U_{\text {before }}-U_{\text {after }}<0 \quad A C C E P T \\
\Delta U=U_{\text {before }}-U_{\text {after }}>0 \text { ACCEPT with probability } e^{\left[-\frac{\Delta U}{k T}\right]}
\end{gathered}
$$

This procedure ensures the system proceeds along a Markov chain ${ }^{3}$ and over a period of time visits a large volume of the available phase space.

The potential energy in EPSR consists of two primary terms. the reference potential energy, $U_{R e f}$, and the empirical potential energy, $U_{E P}$. $U_{R e f}$ takes on a standard form and reference values may be taken from literature depending on the system in question. This potential is used throughout the EPSR simulation, but at the onset on its own to get the simulation box into a likely region of phase space for the system being studied. This ensures that there are sensible molecular geometries and no atomic overlaps. Once the simulation with the reference potential alone has come to equilibrium, $U_{E P}$ is used to guide the atomic and molecular moves in directions that give the closest representation of the diffraction data.

The total potential of the system is represented as $U=U_{R e f}+U_{E P}$. Both of these potential energy terms can be spilt into terms relating to the separation of the individual atoms and molecules, with atoms of different types having different interaction potentials.

For example, the potential energy between atoms of type $i$ and type $j$ separated by a distance $r$ would be given by

$$
U_{i j}(r)=U_{i j}^{(R e f)}(r)+U_{i j}^{(E P)}(r),
$$

\footnotetext{
${ }^{3} \mathrm{~A}$ Markov chain is a sequence of trials that satisfies two conditions: (i)The outcome of each trial belongs to a finite set of outcomes, called the state space, (ii)The outcome of each trial depends only on the outcome of the trial that immediately precedes it.
} 


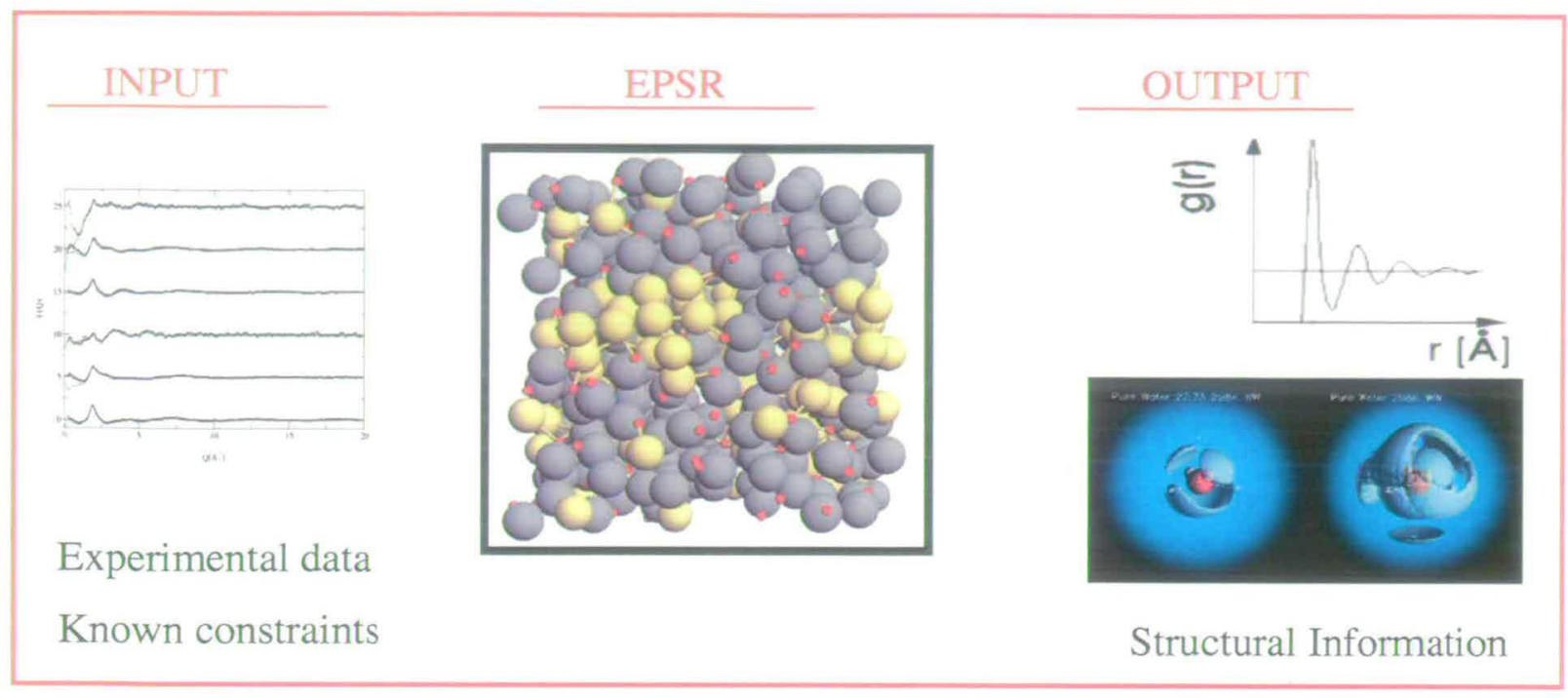

Figure 4.8: Flowchart illustrating the main steps involved in the Empirical Potential Structural Refinement(EPSR)

with the total potential energy of the system being given by

$$
U=\frac{1}{2} \sum_{i} \sum_{j \neq i} U_{\alpha(i) \alpha(j)}\left(r_{i j}\right),
$$

where $r_{i j}$ is the separation of atoms $i, j$ and $\alpha(i)$ represents the type of atom $i$, i.e. whether it is carbon, hydrogen, oxygen etc. The factor of $\frac{1}{2}$ in the equation is needed to prevent double counting of atom pairs, and the summation proceeds over all atom pairs in the system.

The EPSR procedure results in an ensemble average of three-dimensional molecular configurations of the mixture exhibiting average structural correlations that are consistent with the available diffraction data. The experimenter now has access to a set of configurations for the system in three dimensions. It is important to discuss some of the finer points of the EPSR procedure. This is necessary to fully understand the mechanics of this important computer modelling technique. 


\subsubsection{Defining the Reference Interatomic Potential}

The starting point of the EPSR simulation is to build an ensemble of molecules whose internal structure reproduces that which can be obtained from the diffraction experiment. Each intra-molecular distance is characterised by an average distance, $d_{\alpha \beta}$, and a width, $w_{\alpha \beta}$, and the intramolecular structure is established by assuming the atoms in each molecule interact via a harmonic potential. The total (dimensionless) intramolecular energy of the system is represented by

$$
U_{\text {intra }}=C \sum_{i} \sum_{\alpha \beta \neq \alpha} \frac{\left(r_{\alpha_{i} \beta_{i}}-d_{\alpha \beta}\right)^{2}}{2 w_{\alpha \beta}^{2}}
$$

where $r_{\alpha_{i} \beta_{i}}$ is the actual separation of the atoms $\alpha, \beta$ in molecule $i$, and $w_{\alpha \beta}^{2}=$ $d_{\alpha \beta} / \sqrt{\mu_{\alpha \beta}}$ with $\mu_{\alpha \beta}=M_{\alpha} M_{\beta} /\left(M_{\alpha}+M_{\beta}\right)$ the reduced mass of the atom pair $\alpha \beta$ and $M_{\alpha}$ the mass of atom $\alpha$ in atomic mass units. $\mathrm{C}$ is a constant determined by comparing the simulated structure factors with the measurements at large Q.

The intermolecular reference potential is based on the Lennard Jones potential plus effective Coulomb charges where appropriate

$$
U_{\alpha \beta}\left(r_{i j}\right)=4 \epsilon_{\alpha \beta}\left[\left(\frac{\sigma_{\alpha \beta}}{r_{i j}}\right)^{12}-\left(\frac{\sigma_{\alpha \beta}}{r_{i j}}\right)^{6}\right]+\frac{q_{\alpha} q_{\beta}}{4 \pi \epsilon_{0} r_{i j}} .
$$

Here $\alpha, \beta$ represent the types of atoms $i, j$ respectively, and if there are $N_{\alpha}$ such atoms in the system, there will be $N_{\alpha}\left(N_{\alpha}+1\right) / 2$ pairs of such interactions. Where the types of the two atoms are different, the well depth parameter, $\epsilon_{\alpha \beta}$ and the range parameter $\sigma_{\alpha \beta}$ are given by Lorentz-Berthelot mixing rules in terms of their values for the individual atoms.

$$
\epsilon_{\alpha \beta}=\left(\epsilon_{\alpha} \epsilon_{\beta}\right)^{\frac{1}{2}}
$$




$$
\sigma_{\alpha \beta}=\frac{1}{2}\left(\sigma_{\alpha}+\sigma_{\beta}\right)
$$

The charges and Lennard-Jones constants for the SPC/E potential of Berendsen et al[87] are used for the water molecules. The H1 potential of Haughney et al is used for methanol molecules[88]. Methanol-water interactions are simulated by LorentzBerthelot mixing rules.

\subsubsection{Running the Simulation}

The simulation is initially run with only the reference potentials $U_{\text {ref }}$. Once the simulation with the reference potential alone has equilibrated, i.e. the calculated structure factors become stationary and no longer change as the simulation proceeds, the empirical potential can be introduced. This is calculated by taking the difference in Q-space between diffraction data $\mathrm{D}(\mathrm{Q})$ and the simulated structure factor, $\mathrm{F}(\mathrm{Q})$, and fitting a function in the form of a series of power exponential functions.

The empirical potential must only represent the true difference between the simulation and diffraction data. Ideally, it should not contain any artifacts associated with the statistical noise, systematic errors and truncation effects of the diffraction data. Since isotope substitution has been performed, there will be several datasets to fit, so that an empirical potential will be generated for each of the site-site distributions of the system.

\subsubsection{The Potential of Mean Force}

The mechanics of EPSR can be understood in terms of the potential of mean force. The potential of mean force is given by 


$$
\psi_{\alpha \beta}(r)=-k_{B} T \ln \left(g_{\alpha \beta}(r)\right) .
$$

If a reasonable first guess potential is known for a system, the potential of mean force can be used to indicate where this model potential needs to be modified to improve agreement with a measured set of site-site pair correlation functions.

The potential of mean force can be linked to the reference potential. Each radial distribution function, whether experimentally(E) determined or modelled(S) has a corresponding potential of mean force

$$
\begin{aligned}
& \psi_{\alpha \beta}^{E}(r)=-k_{B} T \ln \left(g_{\alpha \beta}^{E}(r)\right), \\
& \psi_{\alpha \beta}^{S}(r)=-k_{B} T \ln \left(g_{\alpha \beta}^{S}(r)\right) .
\end{aligned}
$$

The negative gradient of this potential is the force between an atom of species $\alpha$ and an atom of species $\beta$ within the system with all the other atoms in place. This is distinct from the force between an $\alpha$ atom and a $\beta$ atom in free space, which can be obtained by taking the negative gradient of $U_{\alpha \beta}$. The reference potential $U_{\alpha \beta}^{0}(r)$ can then be coupled with a perturbation derived from the difference between the potentials of mean force

$$
U_{\alpha \beta}^{N}(r)=U_{\alpha \beta}^{0}(r)+\left(\psi_{\alpha \beta}^{E}(r)-\psi_{\alpha \beta}^{S}(r)\right)=U_{\alpha \beta}^{0}(r)+k_{B} T\left(\ln \left(g_{\alpha \beta}^{S} / g_{\alpha \beta}^{E}(r)\right)\right) .
$$

This new potential $U_{\alpha \beta}^{N}(r)$ can then be used to run a simulation, this in turn can then be used to generate a new $\psi_{\alpha \beta}^{S}(r)$, which in turn can be used to calculate a new 'new' potential. 


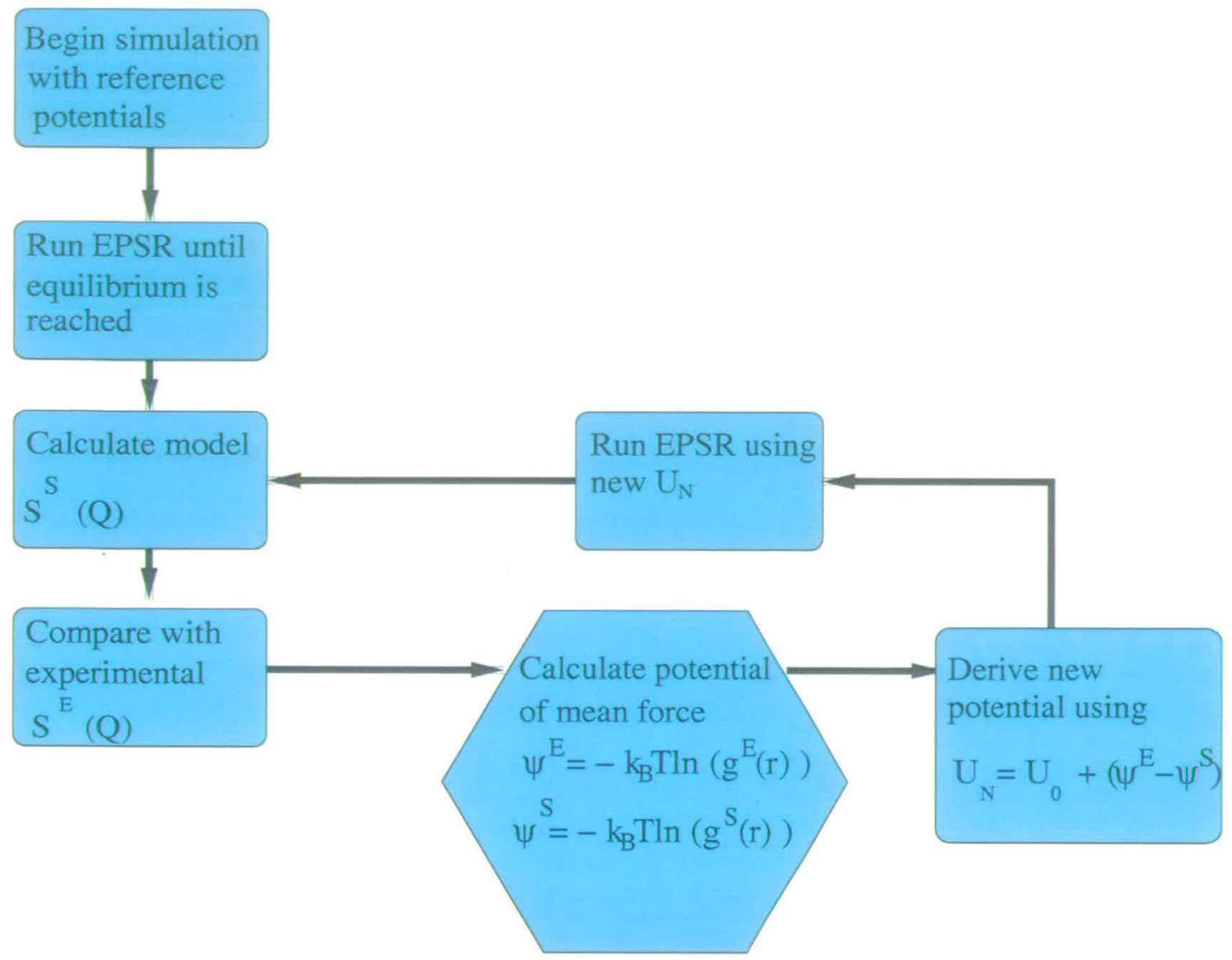

Figure 4.9: Flowchart illustrating the main steps involved in the Empirical Potential Structural Refinement (EPSR)

\subsubsection{Summary of EPSR}

The algorithm to run EPSR is summarised in Figure 4.9. Once the empirical potential has stopped changing, or the absolute energy of the empirical potential has reached its limit, the simulation can be used to extract ensemble averages of required quantities. EPSR is a valuable tool for extracting useful information from the diffraction data. In the next four chapters some exciting new results, obtained from EPSR, will be presented in detail. 


\section{Chapter 5}

\section{Bi-Percolating Liquid Mixture}

\subsection{Motivation}

The availability of experimental data only at dilute and concentrated alcohol limits[13, 12], and the apparently contradictory results from computer simulation[32, 33], [37, $39,40]$ has provided a strong motivation to undertake a systematic survey of the methanol-water system. The extended structure (clustering) as a function of concentration in the model aqueous methanol system was explored using neutron diffraction and Empirical Potential Structural Refinement (EPSR) performed at specific state points. This chapter presents this very interesting work, exploring the changes in the clustering behaviour as a function of concentration. The chapter begins with an account of the experimental details and EPSR modelling procedure. Then the cluster distributions are presented and discussed in relation to percolation theory. 


\subsection{Experimental Details}

Protiated and deuterated samples of methanol and water were obtained from SigmaAldrich and used without additional purification. Neutron diffraction measurements were performed on the SANDALS time-of-flight diffractometer on the ISIS pulsed neutron source at the Rutherford Appleton Laboratory, U.K. SANDALS is especially built to investigate the structure of liquids and amorphous materials. The combination of an intense pulsed neutron source and a large number of detectors at low angles make it particularly useful for measuring structure factors containing light atoms such as hydrogen and deuterium. Isotopically substituted, but otherwise similar mixtures of methanol and water were prepared with the correct methanol-water molecular ratio.

A total of 7 samples were measured for methanol mole fraction $x=0.27, x=0.54$ and $x=0.70$, these were:

1. $C D_{3} O D$ in $D_{2} O$

2. $\mathrm{CD}_{3} \mathrm{OH}$ in $\mathrm{H}_{2} \mathrm{O}$

3. A 50:50 mixture of 1 and 2

4. $\mathrm{CH}_{3} \mathrm{OD}$ in $\mathrm{D}_{2} \mathrm{O}$

5. A 50:50 mixture of 1 and 4

6. $\mathrm{CH}_{3} \mathrm{OH}$ in $\mathrm{H}_{2} \mathrm{O}$

7. A $50: 50$ mixture of 1 and 6

For $x=0.05,5$ samples were measured, these were:

1. $C D_{3} O D$ in $D_{2} O$

2. $\mathrm{CD}_{3} \mathrm{OH}$ in $\mathrm{H}_{2} \mathrm{O}$ 
3. A $50: 50$ mixture of 1 and 2

4. $\mathrm{CH}_{3} \mathrm{OH}$ in $\mathrm{H}_{2} \mathrm{O}$

5. A 50:50 mixture of 1 and 4

In order to learn more about methanol-methanol interactions, the solute-solute partial structure factor was obtained by isotope substitution on the methyl hydrogens using sample 1,2 , and 3 .

Isotope substitution on the hydroxyl hydrogens of water and methanol in samples 1, 4, and 5 gave the correlations between hydroxyl hydrogens.

Samples 1,6 , and 7 provide measure of the correlations between all the hydrogens in the solutions.

Samples were placed in flat plate cells made from a Ti-Zr alloy that gives negligible coherent scattering. These were mounted on a closed cycle refrigerator, and neutron diffraction measurements were made at temperatures of $293 \mathrm{~K}$ (with mole fraction $x=0.05, x=0.27, x=0.7)$ and $298 \mathrm{~K}(x=0.54)$ respectively. Corrections for attenuation and multiple scattering were made using the ATLAS program suite (see Chapter 4). A further correction for inelastic scattering was also made[84]. The differential scattering cross-section for each sample was obtained by normalising to a vanadium standard sample. These procedures lead to a structure factor $F(Q)$ having the form $F\left(S_{\mathrm{HH}}(Q), S_{\mathrm{XH}}(Q), S_{\mathrm{XX}}(Q)\right)$ where $\mathrm{S}_{H H}(\mathrm{Q})$ gives correlations between labelled atoms and $\mathrm{S}_{X H}(\mathrm{Q})$ and $\mathrm{S}_{X X}(\mathrm{Q})$ are the two composite partial structure factors which give the remaining correlations between other types of atoms $(X)$ and the labelled atom type $(\mathrm{H})$ in the form of a weighted sum of individual site-site partial structure factors.

The four neutron diffraction experiments that have been described where carried out by Dr Sanhita Dixit ( $x=0.70, x=0.27, x=0.05)$ and Dr Valarie Réat $(x=0.54)$. 
All of the modelling and analysis, using the Empirical Potential Structural Refinement procedure, was carried out by the author of this thesis. This will now be described in detail below.

\subsection{Structure Factor Fits}

A comparison between the experimentally-measured partial structure factors (circles) and those generated from the ensemble-averaged EPSR configurations (lines) is shown in Figure 5.1 for methanol-water mole fraction $x=0.54$. A total of 600 molecules (methanol and water) were contained in a cubic box of the appropriate dimensions to give the measured density of each solution. The constraints used in the EPSR modelling for each concentration of methanol water solution are shown in Table 5.1. The data shown are the interference differential scattering cross-sections for the samples (i) through (vii) described under the Experimental Details section. Discrepancies are observed in the low $Q$ region. These are caused by difficulties in removing completely the effect of nuclear recoil from the measured data. However, this recoil effect is expected to have only a monotonic dependence on $Q$ and so is unlikely to influence the model structure to any significant extent. Similar structure factor fits were obtained for all of the methanol-water concentrations studied; methanol mole fraction $x=0.70$, $x=0.27$ and $x=0.05$.

\subsection{Micro-segregation}

Using EPSR a snapshot of the simulation box showing the experimentally constrained system can be obtained. Visual inspection of the boxes of atoms reveals significant segregation of water from methanol at all concentrations. An example is shown for methanol mole fraction $x=0.54$ in Figure 5.2. Methyl groups are shown as black 


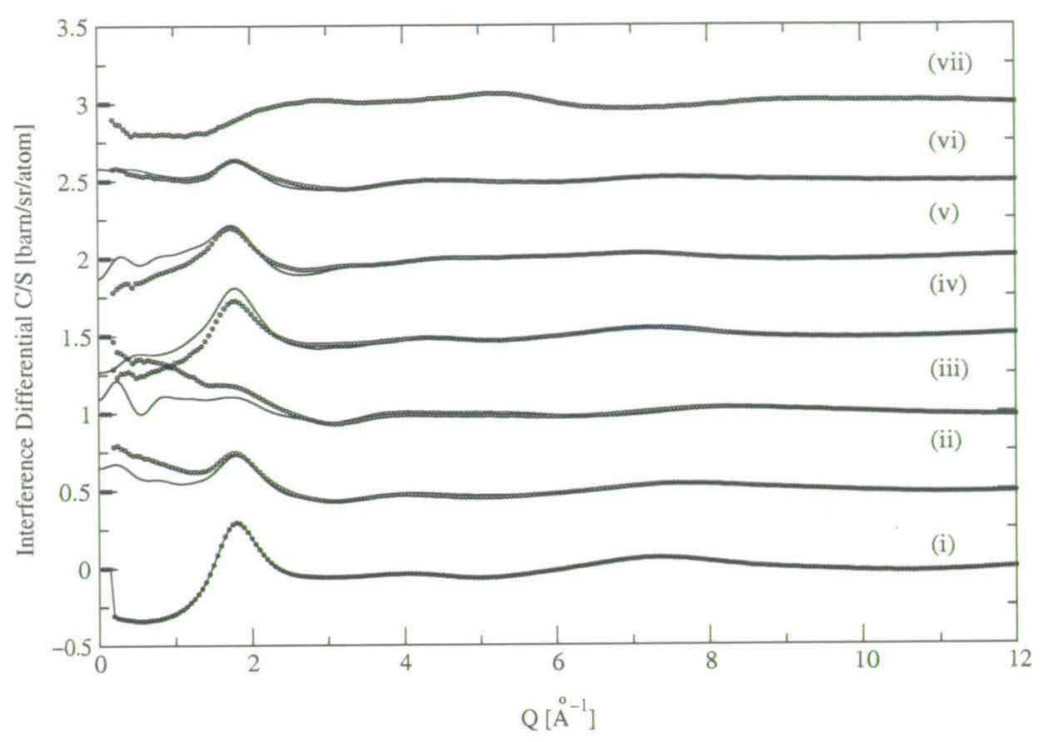

Figure 5.1: Typical example of the fits (lines) obtained by the EPSR computer simulation procedure compared to the original data (circles). The data shown in this case $(x=0.54)$ are the interference differential scattering cross-sections for the samples (i) through (vii) described under the Experimental Details section

\begin{tabular}{|c|c|c|c|c|c|c|}
\hline $\begin{array}{c}\text { Mole fraction } \\
\mathrm{x}\end{array}$ & $\begin{array}{c}\text { Temp. } \\
\mathrm{K}\end{array}$ & $\begin{array}{c}\text { Total No. } \\
\text { molecules }\end{array}$ & $\begin{array}{c}\text { No. methanol } \\
\text { molecules }\end{array}$ & $\begin{array}{c}\text { No. water } \\
\text { molecules }\end{array}$ & $\begin{array}{c}\text { No. density } \\
\text { atoms/ } \AA^{3}\end{array}$ & $\begin{array}{c}\text { Box Size } \\
\AA\end{array}$ \\
\hline 0.05 & 293 & 600 & 30 & 570 & 0.0995 & 26.68 \\
\hline 0.27 & 293 & 600 & 162 & 438 & 0.0967 & 28.69 \\
\hline 0.54 & 298 & 600 & 324 & 276 & 0.0955 & 30.73 \\
\hline 0.70 & 293 & 600 & 420 & 180 & 0.0930 & 32.04 \\
\hline
\end{tabular}

Table 5.1: Parameters used in Empirical Potential Structural Refinement 


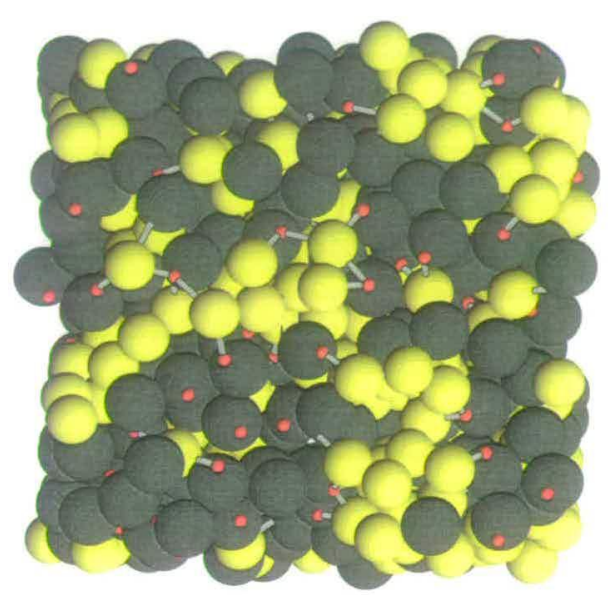

Figure 5.2: Snapshot of an experimentally-constrained EPSR model of the methanol-water mixture at $x=0.54$ showing clusters of the segregated components. Methyl groups are shown as black spheres, large yellow spheres highlight the position of water molecules and small red spheres denote methanol oxygen atoms.

spheres, large yellow spheres highlight the position of water molecules and small red spheres denote methanol oxygen atoms. Visual inspection also suggests that the methanol clusters do not tend to form hydrogen-bonded chains to the same extent as in the pure alcohol. Instead the methyl headgroups tend to be in contact, with the hydroxyl headgroups bonding to water molecules forming the main boundary between methanol- and water-rich regions. This is broadly as expected for a hydrophobicallydriven system and is what has been observed in earlier diffraction work[27, 89].

\subsection{Defining the Clusters}

In analysing the experimentally-constrained EPSR configurations, a particular definition of a cluster was made based on bond connectivity. Specifically, water molecules are assigned to the same cluster if they can be connected by a continuous hydrogenbond network. The criterion used is that two water molecules are said to be hydrogen- 
bonded if their constituent oxygen atoms are less than $g_{O_{W} O_{W}}$ apart, where $g_{O_{W} O_{W}}$ is determined as the minimum following the first peak in the water oxygen-water oxygen pair correlation function (approximately $3.5 \AA$ for EPSR-fitted functions). For the methanol molecules, clusters may be defined in one of two ways. When investigating hydrogen-bonded clusters, the same criterion was used as for water, i.e. if constituent methanol oxygen atoms are less then $g_{O O}$ apart. However, the clustering of methanol molecules via methyl group association was also investigated. In this case, two methanol molecules are assigned to the same cluster if the $g_{C C}$ distance is less than the minimum following the first peak determined from the methanol carbon-methanol carbon pair correlation function (which is approximately $5.7 \AA$ ). According to this criterion, methanol molecules that are in contact only via their non-polar groups are not within the same (hydrogen-bonded) cluster.

\subsection{Percolation Theory}

Consider immersing a porous stone in a bucket of water. What is the probability that the centre of the stone will be wetted? To answer this question we can use something called percolation theory which allows modelling of a random medium. In this section the basic principles of percolation theory will be introduced[90]. It will in no way attempt to cover the entire theory but key issues will be clearly presented and illustrated. A number of steps most be followed. Imagine the stone in question can be considered as a large array of squares. This array is considered to be so large that any effects from its boundaries are negligible.

If one of squares in the lattice contains water then it is said to be 'occupied'. If a square is empty it is said to be 'vacant'. A cluster can be defined as a group of neighbouring squares which are occupied or contain water. From Figure 5.3 it can be seen that squares are called neighbours if they have one side in common but not if they only 

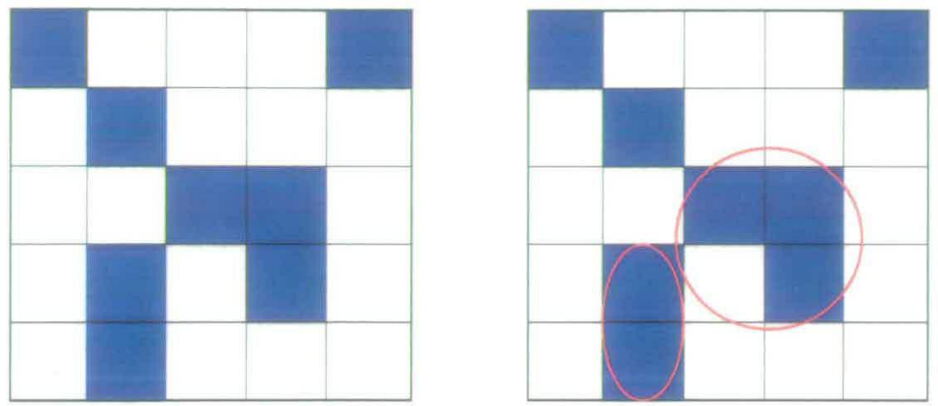

Figure 5.3: Illustration of a square lattice with some occupied sites (left) leading to some clusters through nearest neighbour sites (right)

touch at one corner. Cells with one side touching are known as "nearest neighbour sites on the square lattice', whereas squares touching at one corner only are known as 'next nearest neighbours'. All sites within one cluster are thus connected to eachother by one unbroken chain of nearest neighbour links from one occupied square to a neighbour square also occupied. Large scale penetration of the porous stone by water is related to the existence of an infinite connected cluster which spans the square lattice. Percolation theory deals with the number and properties of these clusters. The questions of interest include; the probability of a spanning cluster, the value of the mean cluster and the strength of the largest cluster. It has been claimed[91] that percolation theory is a cornerstone of the theory of disordered media. It can be used to study a diverse range of disordered systems from epidemics to fires in orchards. In the example of the stone in a bucket of water, the sites were occupied if water was present. In other systems the occupation will be specific to the system. For example, in a binary mixture of liquid A and liquid B, a site on a square lattice could be defined as occupied if it contained liquid $\mathrm{A}$ and vacant if it contained liquid $\mathrm{B}$.

Simulations are excellent indicators of the likely structure of the lattice. A number of simulations can be run for different probabilities of site occupation. The output is then a distribution of cluster sizes for each probability (c.f. For the example of a binary liquid mixture this probability corresponds to the concentration of say liquid A). For example Figure 5.4 shows a distribution of clusters for a probability, $p=0.28$, where 

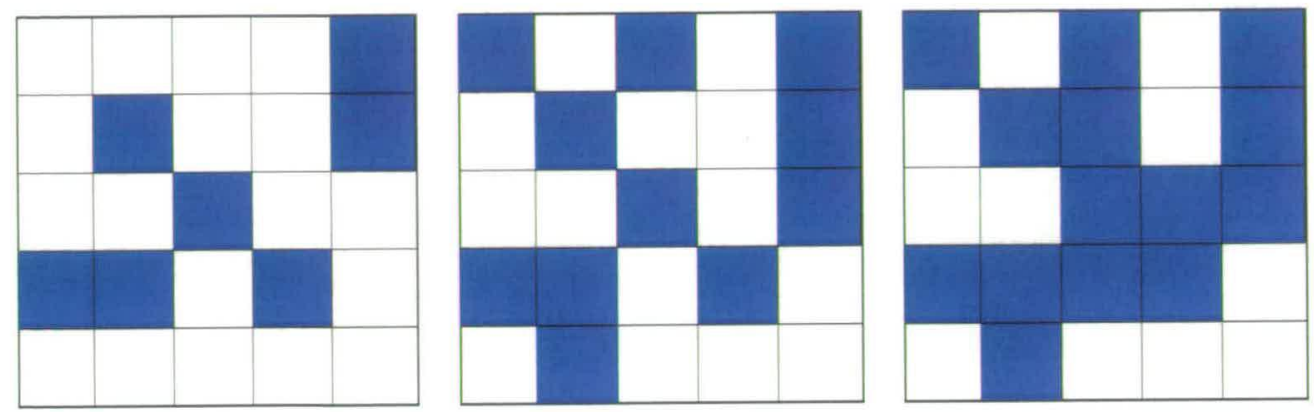

Figure 5.4: Illustration of systems with increasing probability of site occupation (left to right) with a fully percolating cluster spanning the dimensions of the box (right)

the clusters are small and isolated (lattice on left). As the probability of site occupation is increased to a value of $p=0.44$, the size of the clusters increase (middle lattice in Figure 5.4). At a critical value of $p$ ( $p=0.56$ for a $2 \mathrm{D}$ square lattice) a cluster forms which pervades the entire lattice. The point at which the system changes from being a lattice of many isolated clusters to that of a spanning cluster is known as the percolation threshold. The behaviour of the system close to this point is very important.

The occurrence of a critical phenomenom is central to the appeal of percolation. In physical terms one might say that the wetting of the stone is a surface effect when the probability is below the percolation threshold, and a volume effect when the probability is above the percolation threshold. The percolation threshold is therefore an important parameter to determine in a disordered system.

Derivation of exact values of the percolation threshold has been possible to date only for certain lattices related to Bethe lattice ${ }^{1}$ and $2 \mathrm{D}$ lattices. Percolation thresholds of 3D networks have been calculated numerically by Monte Carlo simulations. One very important example is that of random site percolation on a 3D simple cubic lattice[92]. Monte Carlo simulations were carried out to determine the percolation threshold for a very large system of lattice length 10001 (this is the largest 3D percolation system ever simulated). The percolation threshold was found to be $p_{c}=0.311600$. It is known that the number of clusters $n_{s}$ is related to cluster size $s$ by a power law decay which

\footnotetext{
${ }^{1}$ A Bethe lattice is an endlessly branching structure without any closed loops
} 
defines the Fisher exponent $\tau$, where

$$
n_{s}\left(p_{c}\right) \propto s^{-\tau}
$$

The Fisher exponent $\tau$ can be found for 2D, 3D and Bethe lattice systems[90]. In the simulation, the number of clusters $n_{s}$ of a certain size $s$ where found at the percolation threshold and these were fitted with a power law with the exponent $\tau=2.2[92]$

The distribution of methanol clusters and water clusters defined previously were plotted using histograms, were the number of clusters of a size $s, n(s)$, was plotted as a fraction of the total number of clusters, $N$, where $N=\sum_{s} n(s)$ (Figure 5.5). It is known that the number of clusters $n_{s}$ is related to cluster size $s$ by a power law decay which defines the Fisher exponent $\tau$. The predicted power law $n_{s} \approx s^{-2.2}$ for random percolation on a 3-d cubic lattice[92] is also shown on Figure 5.5, illustrated as a dashed line. Clusters lying to the right-hand-side of this dashed line are then known as percolating clusters, while those lying to the left of this dashed line are known as non-percolating clusters. A percolating cluster is one which spans all three dimensions of the simulation box, i.e. crosses all 6 sides of the box.

The dependence of cluster distributions in relation to the size of the system employed was investigated. Using different system sizes, it was found that the cluster distributions were practically identical in all cases and therefore it seems that observations of clusters are not significantly dependent on the choice of system size.

\subsection{A Bi-Percolating Liquid Mixture}

The dependence of cluster distributions was investigated for four different solutions of methanol-water mole fraction $x=0.05, x=0.27, x=0.54$ and $x=0.70$. The clustering of both species, methanol and water, were explored quantitatively as a function of concentration. For water molecules the hydrogen-bond definition was used to 


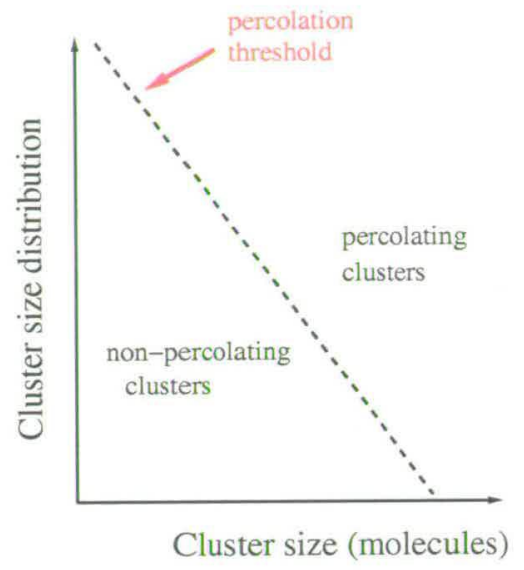

Figure 5.5: Schematic of cluster size distribution showing the cluster size (number of molecules) as a function of cluster size distribution. Also shown is the predicted power law $n_{s} \approx s^{-2.2}$ for random percolation on a 3-d cubic lattice[92].

designate which molecules belong to a given water cluster, while for methanol clusters the $\mathrm{C}-\mathrm{C}$ distance definition was used, as this criterion is more indicative of the nature of the methanol clustering than the hydrogen bond criterion.

The cluster size distributions as obtained from the EPSR ensembles (for $x=0.7,0.54$, 0.27 and 0.05 ) are shown in Figure 5.6. The predicted power law $n_{s} \approx s^{-2.2}$ for random percolation on a 3-d cubic lattice[92] is also shown on Figure 5.6, illustrated as a dashed line. Cluster size distributions for water clusters are shown on the right and methanol clusters are shown on the left.

It can be seen that for the water clusters, percolating clusters are achieved at concentrations of methanol-water mole fraction $x=0.05$ and $x=0.27$. At $x=0.54$ the clusters size distribution also crosses the percolation threshold. For methanol clusters, percolating clusters are achieved at concentrations of methanol-water mole fraction $x=0.70, x=0.54$ and $x=0.27$.

Several "special" concentrations therefore emerge as defined by changes in cluster- 

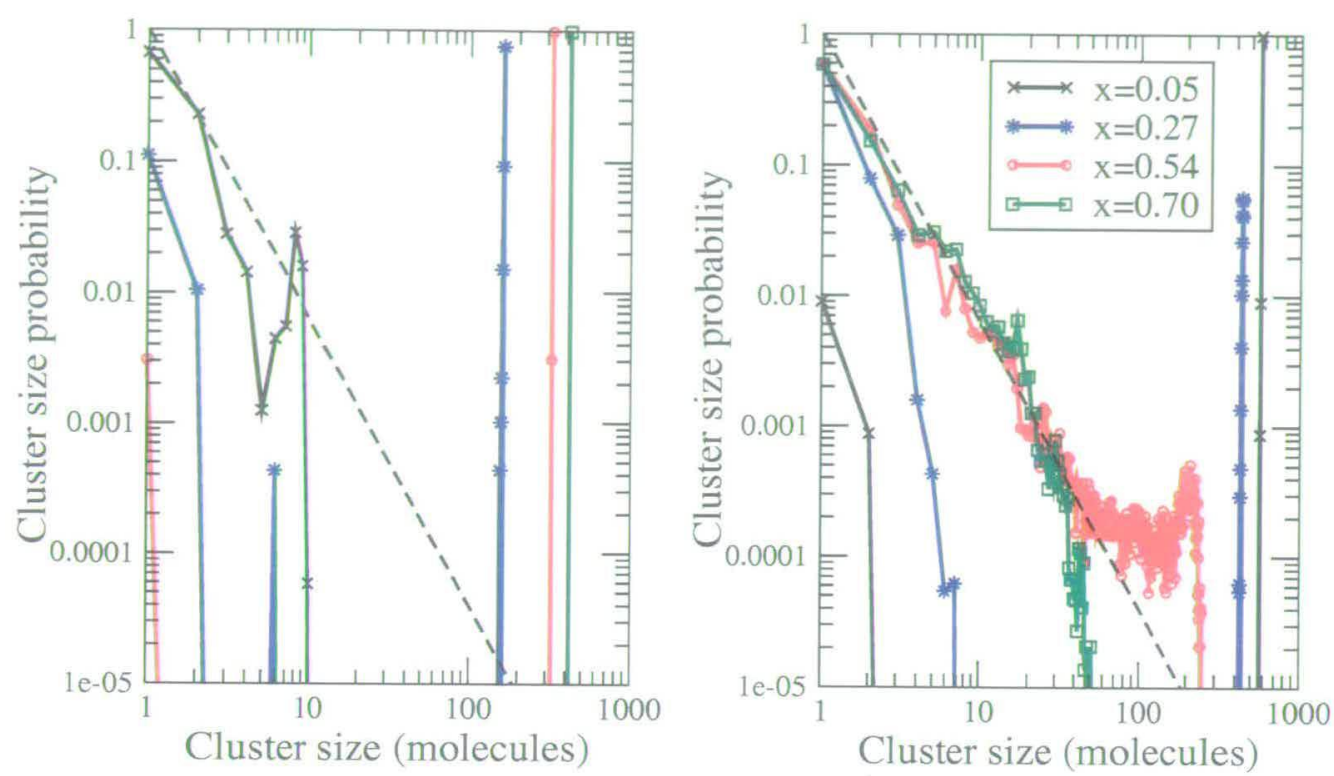

Figure 5.6: Cluster size distributions for water (right) and methanol (left) clusters in methanolwater mixtures mole fractions $0.05,0.27,0.54$ and 0.7 . The dashed lines show the predicted cluster size distribution at the percolation threshold[92]. Percolation in the simulated box occurs when clusters of a size close to the number of molecules in the simulation box form (vertical lines on the right hand side of the plot).

ing behaviour. Specifically, $x \approx 0.27$ determines the approximate alcohol concentration below which water percolates throughout the mixture while methanol does not - occurring instead only in small, isolated clusters. Above this molar fraction, however, methanol percolates throughout the mixture. The larger water clusters also percolate (at $x=0.54$, see Figure 5.6 ) but become increasingly isolated until they are confined to distinct, non-spanning clusters by $x=0.7$. Thus, according to the experimentally-constrained EPSR data in the approximate concentration range defined by $0.27<x<0.54$ both water and alcohol clusters percolate simultaneously, making this a bi-percolating liquid mixture. 


\subsection{Discussion}

Despite their structural simplicity, it is well known that the thermodynamic and transport properties of alcohol-water mixtures, such as the mean molar volume, the diffusion coefficient, the compressibility and the excess entropy, are significantly smaller, and the viscosity significantly larger, than the values that might be expected from an ideal mixture of the pure liquids[71, 93, 94, 95, 96, 97]. The longstanding explanation of these effects in terms of an enhanced structuring of the water in the presence of the alcohol[10] does not appear to be supported by modern diffraction experiments $[26,13,98]$ and an alternative model is needed.

Recent neutron diffraction studies of alcohol-water binary mixtures[13, 89, 27, 12] are leading to new insights into the behaviour of water near molecules containing both hydrophobic and hydrophilic groups. These have established that, in the dilute alcohol limit, the alcohol molecule appears to have a mildly compressive effect on the water structure, as is seen from the slight inwards movement of the second peak of the wateroxygen radial distribution function compared to the same function in pure water. This second peak, which occurs near $r \approx 4.5 \AA$ in the $O_{W} O_{W}$ radial distribution function of pure water, has widely been interpreted as the signature of the tetrahedral ordering in water. By contrast, in the opposite (concentrated alcohol) limit, the system segregates into what is effectively a molecular-scale microemulsion[12], with methyl head groups pushed toward each other, and the hydrophilic hydroxyl groups forming a boundary around small pockets of a water-like fluid.

These simple systems have also been the subject of considerable computational investigations. The earliest of these $[32,33,37]$ used Monte Carlo methodologies at low or infinitely dilute concentrations of alcohol. Despite different computational models, and some apparent contradictions between their results, they all found an enhanced cage-like structure of water around the methyl group, in accordance with the Frank and Evans model[10]. Recently however, Meng and Kollman [39] have performed 
MD simulations of various solutes (including methanol) at infinite dilution and found that the water structure around the hydrophobic groups is preserved rather than enhanced. Ab initio simulations of alcohol-water mixtures have also recently been reported $[41,99]$, however the computational expense of these simulations is such that they are restricted to picosecond simulation on small system sizes. Nonetheless, these studies have also pointed to the lack of structural enhancement of the water surrounding the hydrophobic moiety in the alcohol. Very recently, results from MD simulations of an alcohol-rich methanol-water solution provides evidence of extreme clustering and micro-immiscibility[100]

Now, through the extensive use of neutron diffraction and EPSR modelling is has been possible to explore the extended structure (clustering) across a range of concentrations in a methanol water system. This work suggests that the nature and extent of clustering in these mixtures may offer a structural explanation for thermodynamic anomalies observed. The micro-segregation in these methanol-water mixtures could be responsible for the maxima and minima observed in particular thermodynamic quantities such as enthalpy and entropy. On the left of figure 5.7 the excess entropies of mixing of alcohols with water at $25^{\circ}$ [71] are shown. For the case of methanol and water the excess entropy is observed to reach a minimum value at methanol mole fraction $x \approx 0.40$. Figure 5.7 (middle graph) also shows the heats of mixing of alcohols with water at $25^{\circ}$ [71]. For methanol and water the heats of mixing goes through an extremum at methanol mole fraction $x \approx 0.33$. Finally the right hand figure 5.7 shows the excess free energies of mixing of alcohols with water at $25^{\circ}$ [71]. The excess free energy reaches it's maximum value at methanol mole fraction $x \approx 0.50$.

Consider now how a random system of 2 liquids might behave which contains methanol 'sized' spheres and water 'sized' spheres, i.e. how would spheres of a size similar to carbon, oxygen and hydrogen pack together in a simulation box and what would happen to the percolation of the system. To do this the hydrogen bonding in the methanolwater system was neglected and the packing was simply considered. When the water 

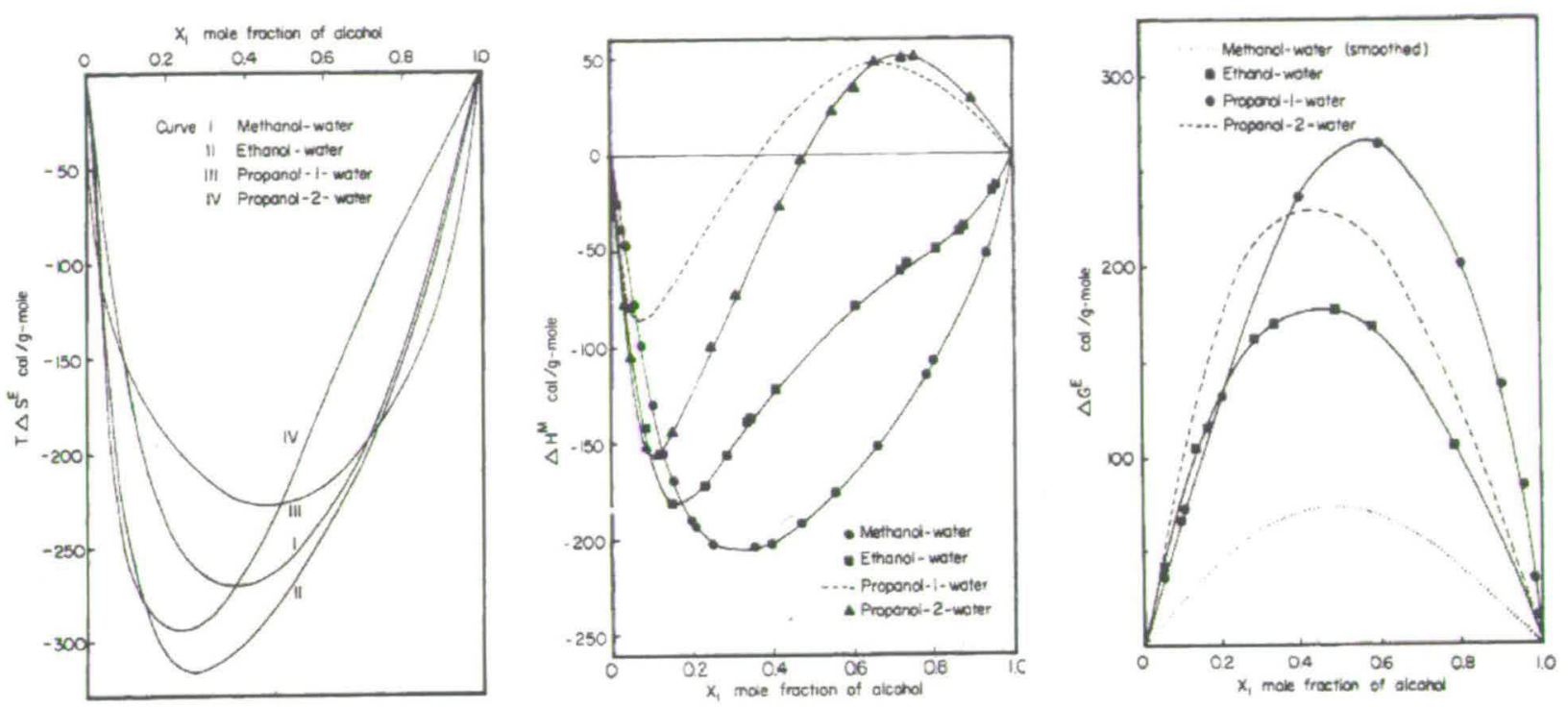

Figure 5.7: Excess entropies of mixing of alcohols with water at $25^{\circ}$ (left), Heats of mixing of alcohols with water at $25^{\circ}$ (middle), Excess free energies of mixing of alcohols with water at $25^{\circ}$ (right)[71]

clusters and methanol clusters were investigated it was found that there were nonpercolating water clusters and percolating water clusters. However the percolating clusters were evident to a much less extent than seen in the EPSR cluster distribution anaysis. although to a lesser degree than in the hydrogen bonded system. Therefore, it seems that in the hydrogen-bonded water system the interactions play a crucial role in the bi-percolation observed.

In earlier work on liquids, computer simulations have identified percolation transitions in supercritical Lennard-Jones fluids[101], supercritical water[102], water in aqueous acetonitrile[103] and aqueous tetrahydrofuran (THF) [104, 105]. However, this is the first report of simultaneous percolation of two fully miscible fluids.

A further feature of the EPSR clusters is revealed in Figure 5.8. The figure shows the ratio of number of water molecules at the surface of a cluster (as defined by being hydrogen bonded to a methanol hydroxyl group) to total number of water molecules in a cluster (right). The ratio of number of methanol molecules at the surface of a cluster to total number of methanol molecules in a cluster is shown on the left. The dashed 


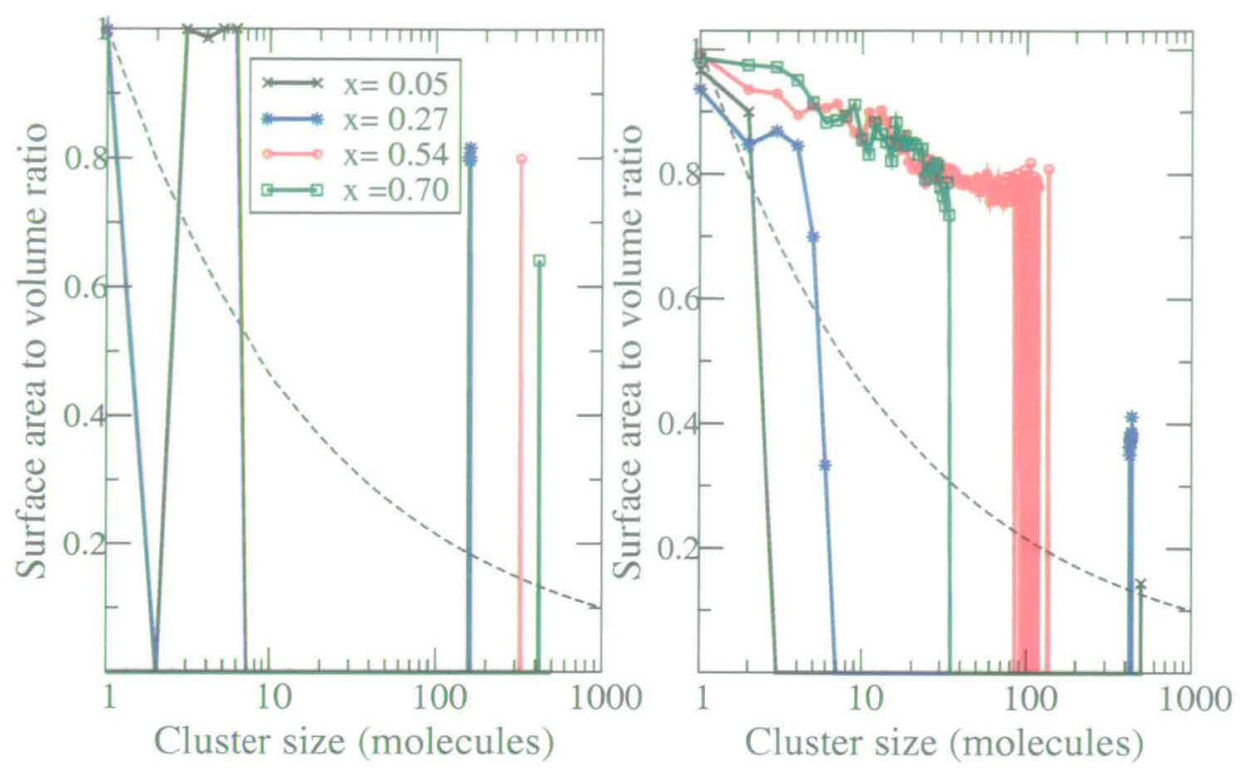

Figure 5.8: Ratio of number of methanol molecules at the surface of a cluster to total number of methanol molecules in a cluster (left). Ratio of number of water molecules at the surface of a cluster (as defined by being hydrogen bonded to a methanol hydroxyl group) to total number of water molecules in a cluster (right). Dashed line shows the $N^{-1 / 3}$ behaviour expected if the clusters grew equally in 3 dimensions with $\mathrm{N}$ the number of molecules in a cluster.

line shows the $N^{-1 / 3}$ behaviour that would be expected for this ratio if the clusters grew equally in 3 dimensions with $\mathrm{N}$ the number of molecules in a cluster.

Only for the fully percolating water cluster at $\mathrm{x}=0.05$ do the clusters show normal $3 \mathrm{D}$ behaviour. For the methanol clusters, even at $x=0.7$ the clusters do not approach full $3 \mathrm{D}$ behaviour. Clearly the ratio does not decay as $N^{-1 / 3}$ as would be expected for a 3-dimensional object: the clusters appear to maximise their surface area by forming as many bonds as possible with methanol. The observed behaviour corresponds much more closely to a 2-dimensional object, suggesting the clusters occur in the form of disordered sheets or cylinders, rather than the sphere-like objects that might be expected in conventional micelle formation. Only at the highest water content, $x=0.05$, do the water clusters appear to have adopted 3D characteristics.

Isotope-labelled neutron diffraction measurements analysed using the empirical potential structural refinement method has been used at particular state points to explore 


\subsection{DISCUSSION}

structure in methanol-water mixtures at several concentrations. The local and extended structures are well described by this method. Highly heterogeneous mixing across the entire concentration range was observed despite apparent miscibility of both components in all proportions. Extended chain, sheet and three-dimensional structures form depending on concentration.

At a particular concentration regime near $x=0.27$ these structures form percolating networks for both components. This concentration has long been considered 'special' as it is near the point where many transport coefficients and thermodynamic functions have extremal values. Other alcohols also show extrema of these same material properties (at lower mole fractions) and the present work suggests a structural basis for these observations connected to the details of mixing heterogeneities. 


\section{Chapter 6}

\section{Cooling Enhances Segregation}

\subsection{Motivation}

Despite the intense activity and success in studying aqueous alcohol systems at room temperature and pressure, there have been no systematic investigations aimed at mapping out the behaviour of the observed extended structures under non-ambient conditions. Therefore, there has been much motivation to explore structural properties of methanol-water mixtures far from the ambient state point. This chapter explores the effects of cooling on the structure and micro-segregation of methanol-water mixtures. The specific objective of the work is to identify the effects of temperature on the structures formed in these solutions and to comment on the nature of intermolecular contacts. Two different concentrations of mixture are presented methanol-water $x=0.27$ and $x=0.54$ mole fraction. The effects of cooling on the water structure will be presented in terms of short range order (local structure) and medium range order (clustering). This work allows exploration of the effects of cooling on local water structure and the water network and could provide an insight into the effects of cooling on more complex biological systems. 


\subsection{Experimental Details}

Isotopically substituted, but otherwise similar mixtures of methanol and water were prepared with the correct methanol-water molecular ratio (mole fraction).

A total of 7 samples were measured for methanol-water $x=0.27$ and $x=0.54$ mole fraction these were:

1. $C D_{3} O D$ in $D_{2} O$

2. $\mathrm{CD}_{3} \mathrm{OH}$ in $\mathrm{H}_{2} \mathrm{O}$

3. A $50: 50$ mixture of 1 and 2

4. $\mathrm{CH}_{3} \mathrm{OD}$ in $\mathrm{D}_{2} \mathrm{O}$

5. A 50:50 mixture of 1 and 4

6. $\mathrm{CH}_{3} \mathrm{OH}$ in $\mathrm{H}_{2} \mathrm{O}$

7. A 50:50 mixture of 1 and 6

Isotope substitution on the hydroxyl hydrogens of water and methanol in samples 1,2 and 3 gave the correlations between hydroxyl hydrogens.

In order to learn more about methanol-methanol interactions, the solute-solute partial structure factor was obtained by isotope substitution on the methyl hydrogens using sample 1,4 and 5 .

Samples 1, 6 and 7 provide a measure of the correlations between all the hydrogens in the solutions.

Samples were placed in flat plate cells made from a $\mathrm{Ti}-\mathrm{Zr}$ alloy that gives negligible coherent scattering. These were mounted on a closed cycle refrigerator, and neutron 
diffraction measurements were made at temperatures of $293 \mathrm{~K}$ and $238 \mathrm{~K}$ (mole fraction $x=0.27)$ and $298 \mathrm{~K}, 260 \mathrm{~K}$ and $190 \mathrm{~K}(x=0.54)$ respectively. Corrections for attenuation and multiple scattering were made using the ATLAS program suite described in the data correction procedure in Chapter 4. A further correction for inelastic scattering was also made[84]. The differential scattering cross-section for each sample was obtained by normalising to a vanadium standard sample.

These procedures lead to a structure factor $F(Q)$ having the form $F\left(S_{\mathrm{HH}}(Q), S_{\mathrm{XH}}(Q)\right.$, $\left.S_{\mathrm{XX}}(Q)\right)$ where $\mathrm{S}_{H H}(\mathrm{Q})$ gives correlations between labelled atoms and $\mathrm{S}_{X H}(\mathrm{Q})$ and $\mathrm{S}_{X X}(\mathrm{Q})$ are the two composite partial structure factors which give the remaining correlations between other types of atoms $(\mathrm{X})$ and the labelled atom type $(\mathrm{H})$ in the form of a weighted sum of individual site-site partial structure factors. All the intermolecular weights for the composite partial structure factors obtained from the neutron diffraction experiment are given in Appendix B.

The neutron diffraction experiments that have been described were carried out by $\mathrm{Dr}$ Sanhita Dixit $(x=0.27)$ and Dr Valarie Réat $(x=0.54)$. All of the modelling and analysis, using the Empirical Potential Structural Refinement procedure, was carried out by the author of this thesis. This will now be described in detail below.

\subsection{Structure Factor Fits}

EPSR produces an ensemble of three-dimensional molecular configurations of the mixture that are consistent with the available diffraction data. A total of 600 molecules (methanol and water) were contained in a cubic box. The ratio of molecules in the simulation box was chosen to maintain the correct mole fraction of the mixture and the box size was fixed to maintain the correct density of the mixture. The values used in the EPSR refinement are shown in Table 6.1. A comparison between the experimentally measured partial structure factors (black circles) and those generated from the 


\begin{tabular}{|c|c|c|c|c|c|c|}
\hline $\begin{array}{c}\text { Mole fraction } \\
x\end{array}$ & $\begin{array}{c}\text { Temp. } \\
/ K\end{array}$ & $\begin{array}{c}\text { Total No. } \\
\text { molecules }\end{array}$ & $\begin{array}{c}\text { No. methanol } \\
\text { molecules }\end{array}$ & $\begin{array}{c}\text { No. water } \\
\text { molecules }\end{array}$ & $\begin{array}{c}\text { No. density } \\
/ \text { atoms } / \AA^{3}\end{array}$ & $\begin{array}{c}\text { Box Size } \\
/ \AA\end{array}$ \\
\hline 0.27 & 293 & 600 & 162 & 438 & 0.0967 & 28.69 \\
\hline 0.27 & 238 & 600 & 162 & 438 & 0.0967 & 28.69 \\
\hline 0.54 & 298 & 600 & 324 & 276 & 0.0955 & 30.26 \\
\hline 0.54 & 260 & 600 & 324 & 276 & 0.0975 & 30.52 \\
\hline 0.54 & 190 & 600 & 324 & 276 & 0.1000 & 30.73 \\
\hline
\end{tabular}

Table 6.1: Parameters of the methanol-water mixtures used in the Empirical Potential Structural Refinement.

ensemble averaged EPSR procedure (red line) with 10000 configurations are shown in Figures 6.1 and 6.2

The fits can be seen to deviate slightly at low $Q$ values. This is due to difficulties encountered in removing the inelastic scattering. However, these deviations correspond to low $\mathrm{Q}$ values and do not affect correlations at intermolecular distances which are of interest in the present work. The EPSR procedure now proceeds to calculate quantities of interest such as the partial radial distribution functions and cluster distributions. 


\subsection{Cooling Enhances Micro-Segregation}

When methanol and water are mixed they form a micro-segregated solution in which the water molecules are found in small 'pockets' or water-rich regions and the methanol molecules are in separate regions[12]. It is very interesting to explore this microsegregation further. This can be done by considering groups of water molecules as water clusters and groups of methanol molecules as methanol clusters. In the previous chapter the cluster distributions of methanol and of water were investigated across the concentration range. It was found that at a particular concentration range both species were percolating in the mixture. Consider now how this bi-percolating mixture behaves as the system is cooled.

The dependence of cluster distributions on cooling was investigated for two different solutions of methanol-water at different temperatures. Firstly, methanol mole fraction $x=0.27$ at $293 \mathrm{~K}$ and $238 \mathrm{~K}$ and methanol mole fraction $x=0.54$ at $298 \mathrm{~K}, 260 \mathrm{~K}$ and $190 \mathrm{~K}$. It is important to note that these are the concentrations at which a bi-percolating mixture was observed. For water molecules the hydrogen-bond definition was used to designate which molecules belong to a given water cluster. The criterion used is that two water molecules are said to be hydrogen-bonded if their constituent oxygen atoms are less than $g_{O_{W} O_{W}}$ apart, where $g_{O_{W} O_{W}}$ is determined as the minimum following the first peak in the water oxygen-water oxygen pair correlation function (approximately $3.5 \AA$ for EPSR-fitted functions).

For methanol clusters the $g_{C C}$ distance definition was used where two methanol molecules are assigned to the same cluster if the $g_{C C}$ distance is less than the minimum following the first peak determined from the methanol carbon-methanol carbon pair correlation function (which is approximately $5.7 \AA$ ).

The cluster size distributions as obtained from the EPSR ensembles (for $x=0.27$ and $x=0.54$ ) are shown in Figure 6.3 for water clusters. The predicted power law $n_{s} \approx s^{-2.2}$ for random percolation on a 3-d cubic lattice[92] is also shown on Figure 
6.3 , illustrated as a dashed line. The number of clusters containing $s$ molecules is plotted as a fraction of total number of clusters, $n(s) / N$ (where $N=\sum_{s} n(s)$ ) against the cluster size $s$.

The water cluster distributions show an enhanced probability of the largest water clusters on cooling, at the expense of medium-sized (100 molecules or so) clusters. The system exhibits larger water clusters and these clusters are more frequently present upon cooling. This is consistent with increased segregation of the two components upon cooling. The same trends are seen in the EPSR analysis of a mole fraction $x=0.27$ mixture, although the effect is less marked since the water clusters are already bigger at this concentration.

The methanol cluster size distributions as obtained from the EPSR ensembles (for $x=0.27$ and $x=0.54)$ are shown in Figure 6.4. 

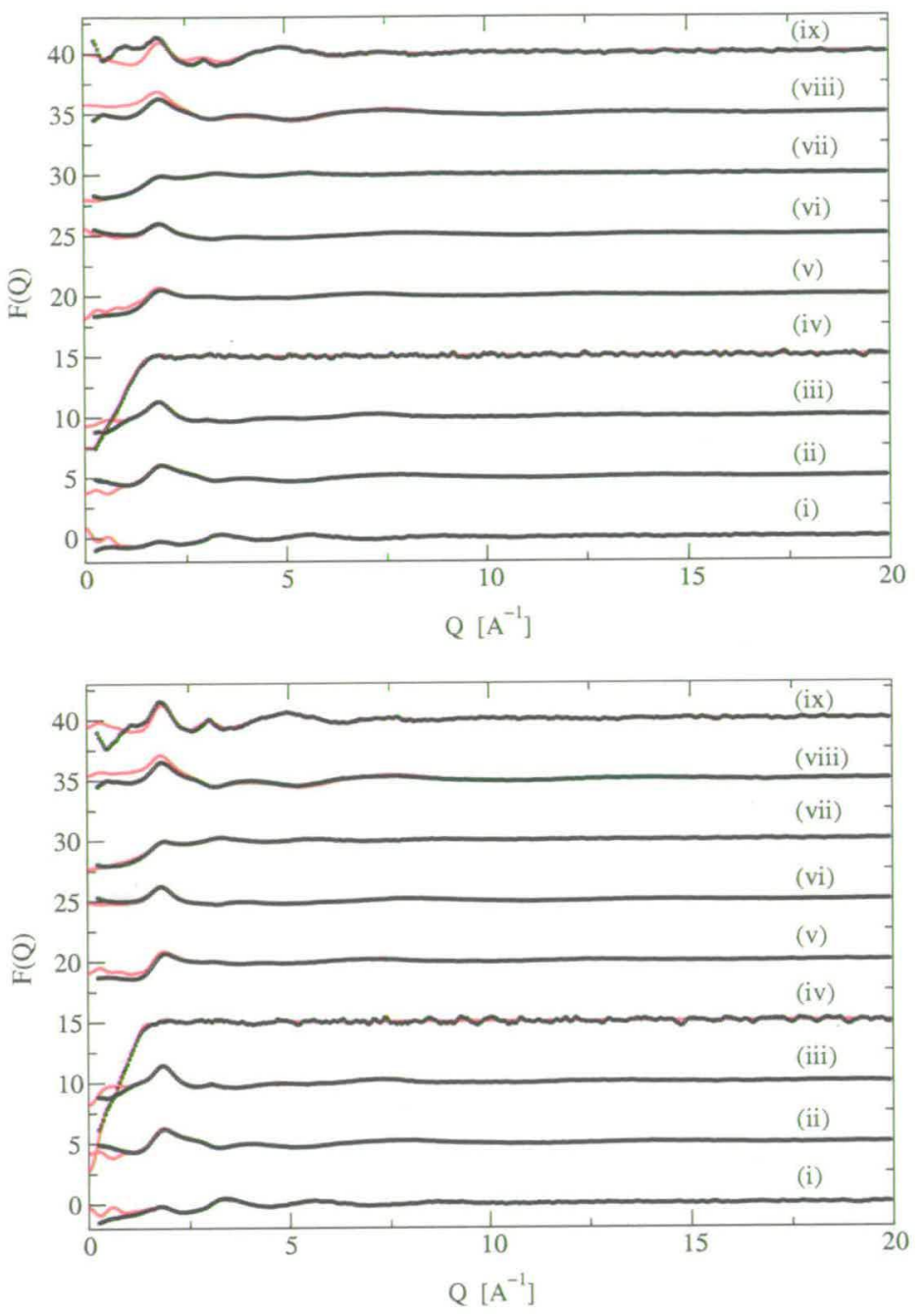

Figure 6.1: Composite partial structure factors fits (red lines) obtained by the EPSR computer simulation procedure compared to the original data (black circles) for methanol-water mixture mole fraction $\mathrm{x}=0.27$ at $298 \mathrm{~K}$ (upper) and $\mathrm{x}=0.27$ at $238 \mathrm{~K}$ (lower) 

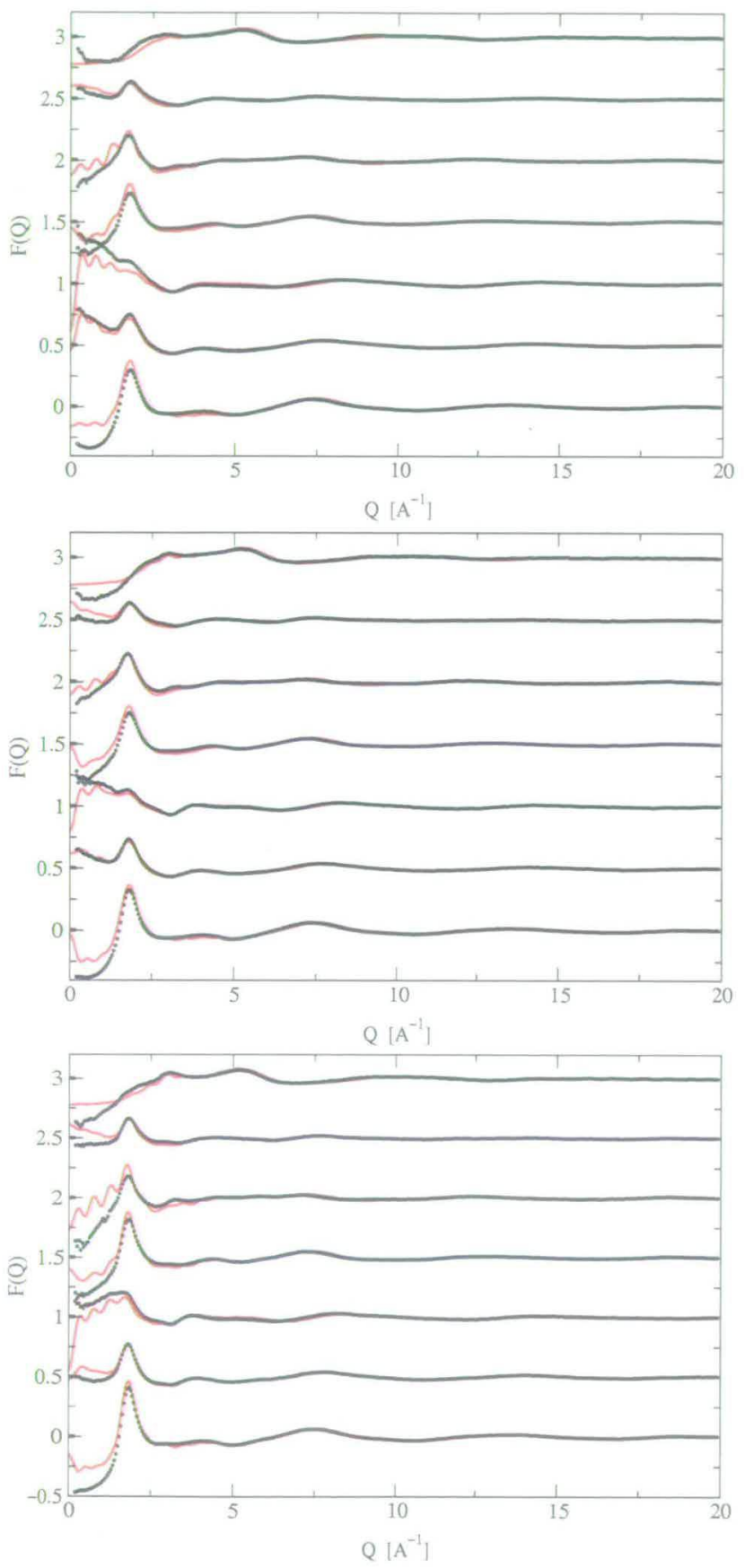

Figure 6.2: Composite partial structure factors fits (red lines) obtained by the EPSR computer simulation procedure compared to the original data (black circles) for methanol-water mixture mole fraction $x=0.54$ at $298 \mathrm{~K}$ (top), $\mathrm{x}=0.54$ at $260 \mathrm{~K}$ (middle) and $\mathrm{x}=0.54$ at $190 \mathrm{~K}$ (bottom) 

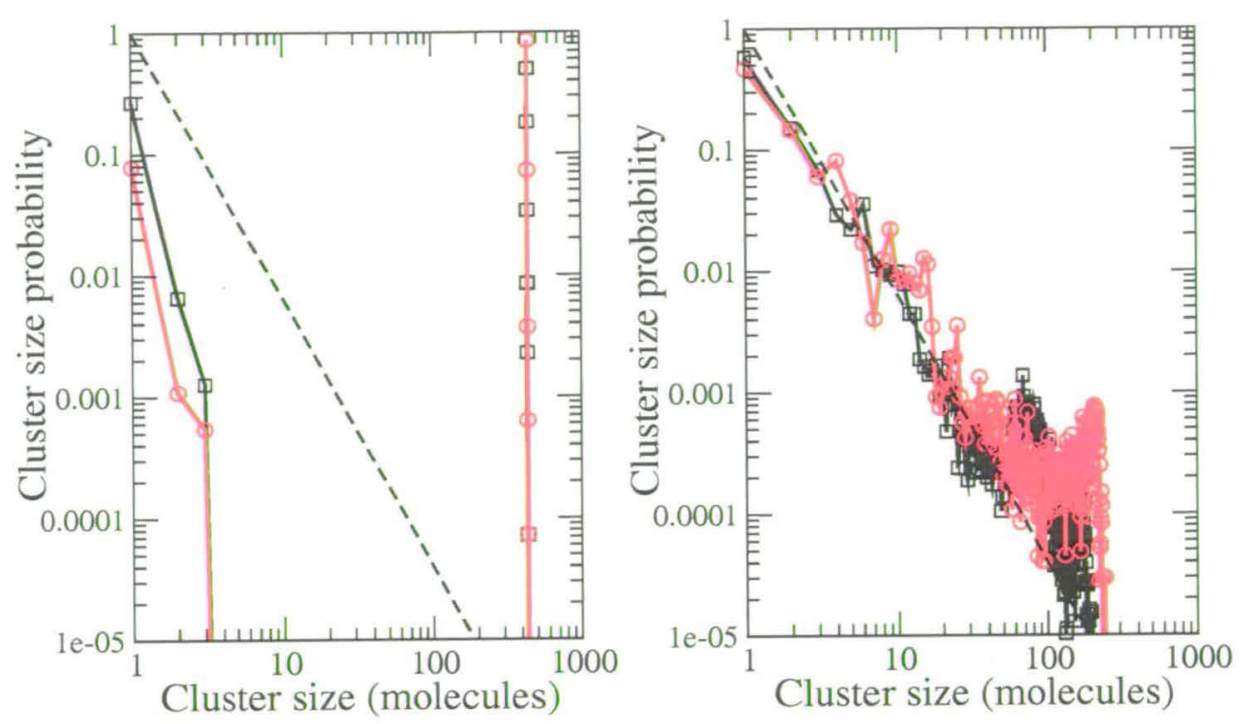

Figure 6.3: Water cluster size distributions in two different methanol-water mixtures, mole fractions $x=0.27$ (left) and $x=0.54$ (right). The black line indicates the system at ambient temperature while the red line indicates the cooled system. The dashed lines show the predicted cluster size distribution at the percolation threshold[92]
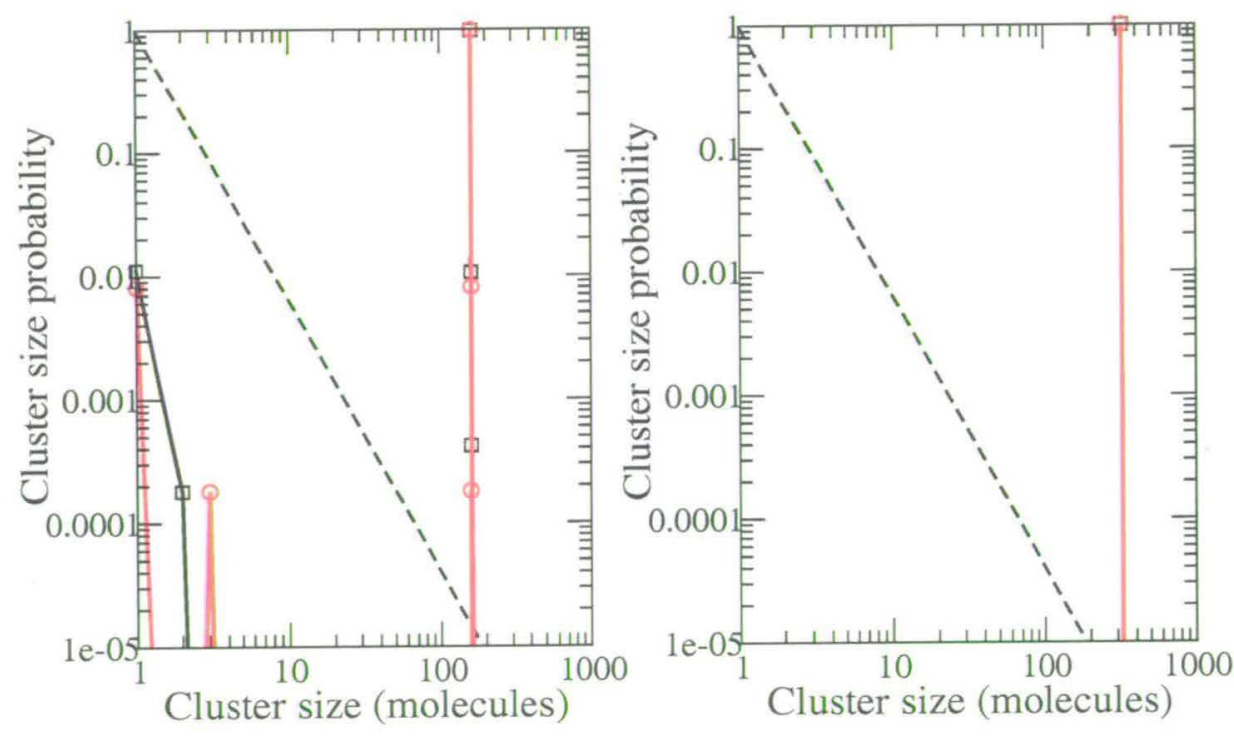

Figure 6.4: Methanol cluster size distributions in two different methanol-water mixtures, mole fractions 0.27 (left) and 0.54 (right). The black line indicates the system at ambient temperature while the red line indicates the cooled system. The dashed lines show the predicted cluster size distribution at the percolation threshold[92] 
By examining the distribution of clusters in the solution it has been found that there is enhanced segregation of methanol and water as the system is cooled. There are more percolating water clusters in the solution on cooling. This enhancement of hydrogen bonding between water molecules can be visualised by producing a 'snapshot' of the methanol-water mixture. The snapshot illustrates one possible scenario or structural arrangement which satisfies the diffraction data. The coordinates of all the atoms in the snapshot are such that the structure factors (Figure 6.2) obtained from the diffraction experiment are satisfied. Figure 6.5 shows a snapshot of an experimentally-constrained EPSR model of the methanol-water mixture at $x=0.54$ at $298 \mathrm{~K}$ (left) and $190 \mathrm{~K}$ (right). Methyl groups are shown as red spheres, large blue spheres highlight the position of water molecules and small grey spheres denote methanol oxygen atoms. The snapshots clearly show clusters of both methanol and water, i.e. a micro-segregated solution.
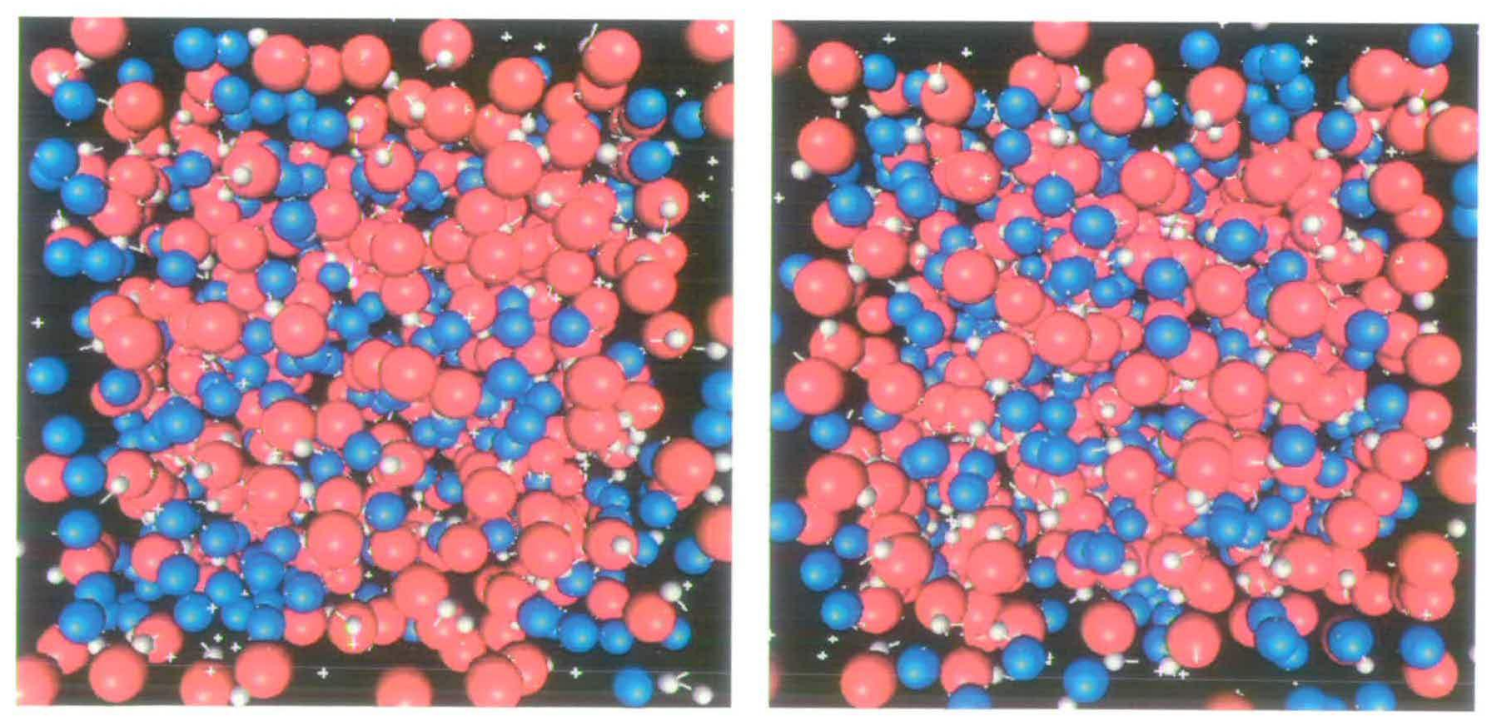

Figure 6.5: Snapshot of an experimentally-constrained EPSR model of the methanol-water mixture at $x=0.54$ at $298 \mathrm{~K}$ (left) and $190 \mathrm{~K}$ (right) showing clusters of the segregated components. Methyl groups are shown as red spheres, large blue spheres highlight the position of water molecules and small grey spheres denote methanol oxygen atoms.

For clarity Figure 6.6 shows the same snapshots of an experimentally-constrained EPSR model of the methanol-water mixture at $x=0.54$ at $298 \mathrm{~K}$ (left) and $190 \mathrm{~K}$ 
(right) where only the water molecules are shown. Again, large blue spheres highlight the position of water oxygen atoms while the small white spheres highlight the position of water hydrogen atoms. Hydrogen bonds between water molecules are illustrated by red dashed lines where the hydrogen bonding criteria was specified based on the location of peaks in the water radial distribution function $g_{O w O w}$. Inspection of the snapshot reveals that the number of hydrogen bonds between water molecules has increased as the $\mathrm{x}=0.54$ methanol water system is cooled. It can also be seen that the water molecules are 'better connected' at low temperature. As the system is cooled the hydrogen bonded network of water molecules has changed from being many small clusters of water molecules to a large inter-connected cluster which spans the simulation box. This is exactly what we would expect to see based on the percolation results presented above. Percolation of water clusters is enhanced in a methanol-water system as the temperature is reduced.
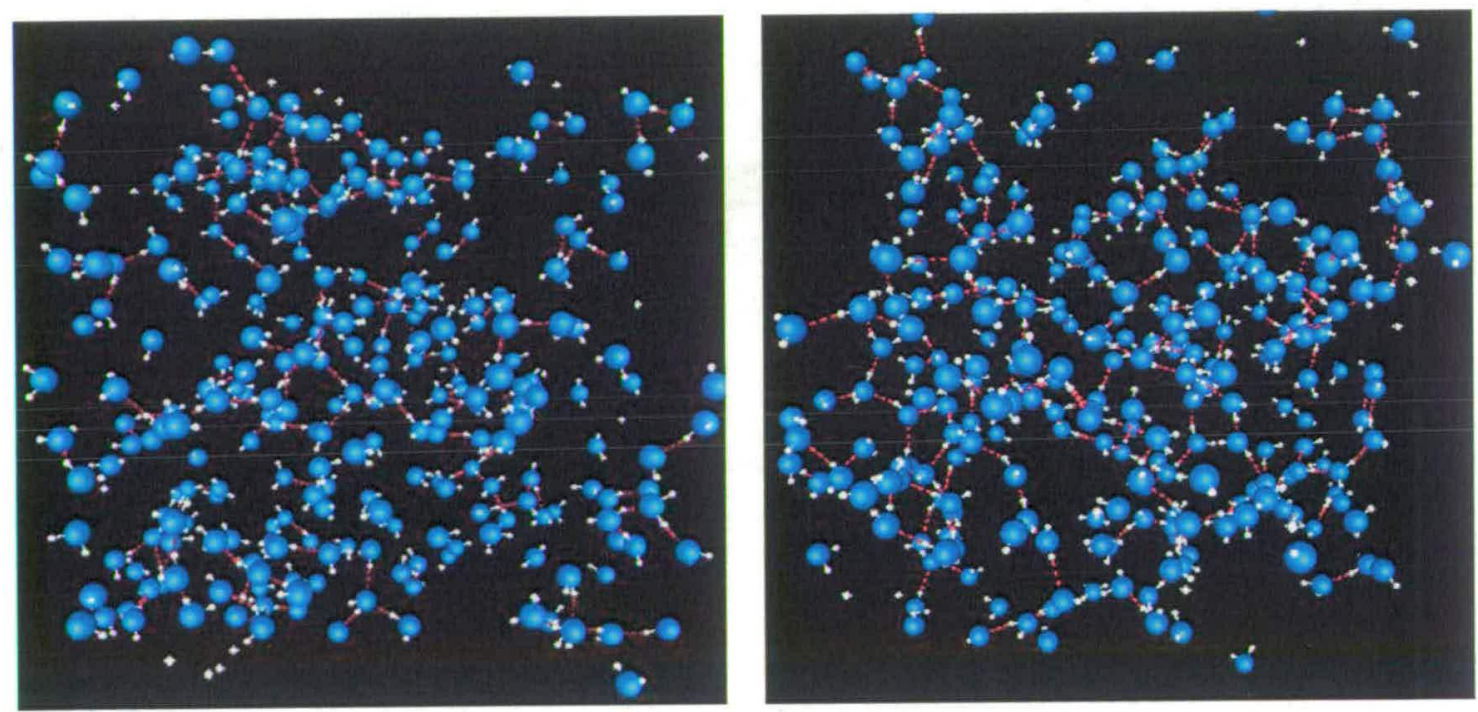

Figure 6.6: Snapshot of an experimentally-constrained EPSR model of the methanol-water mixture at $x=0.54$ at $298 \mathrm{~K}$ (left) and $190 \mathrm{~K}$ (right) were only the water molecules are shown, highlighted as large blue spheres (water oxygen) and small white spheres (water hydrogens). Hydrogen bonding between water molcules is illustrated with a dashed red line

The increased water clustering with cooling observed in the methanol water cluster distribution plots means that there is less water alcohol mixing and therefore increased 
hydrophobicity. The water is 'seeing' more of itself and less of the alcohol. From the accepted view of hydrophobicity, methyl group association is seen as a signature of hydrophobicity. Therefore, there must also be increased alcohol-alcohol contacts although this cannot be seen in the methanol cluster distributions since the alcohol clusters are already fully percolating.

Therefore, it can be concluded that on cooling the methanol-water mixture in the concentration range $x=0.27-0.54$ the hydrophobicity of the system is increasing.

\subsection{Enhanced Hydrogen Bond Interactions}

Cluster distributions have given a strong indication that micro-segregation observed in methanol-water systems is enhanced on cooling. Consider now the local structure information gained from neutron diffraction experiments where other indications of this enhancement of micro-heterogeneity might be found. Another way to learn more about the system is to consider the hydrogen bond interactions. It is important to look at the number of water molecules hydrogen bonding to methanol molecules, water molecules hydrogen bonding to water molecules and methanol molecules hydrogen bonding to methanol molecules. This can be done by considering the local structure of the molecules in the solution and examining a number of partial radial distribution functions (RDF's) and determining the coordination numbers in the surrounding coordination shell ${ }^{1}$.

Consider first the methanol-water hydrogen bonding interactions. To do this it is necessary to examine two partial radial distribution functions, $g_{O H w}(r)$ and $g_{H O w}(r)$. Figure 6.7 shows these RDF's for the two systems under investigation. On the top panel $g_{O H w}(r)$ is shown for methanol-water $\mathrm{x}=0.27$ mole fraction (left) and $\mathrm{x}=0.54$ mole fraction (right) and on the bottom panel $g_{H O w}(r)$ is shown for methanol-water $\mathrm{x}=0.27$

\footnotetext{
${ }^{1}$ The coordination number is the number of molecules which exist in a particular coordination shell
} 
mole fraction (left) and $\mathrm{x}=0.54$ mole fraction (right). The room temperature measurement is shown as a solid black line while the cooled system $(x=0.27$ at $238 \mathrm{~K}$ and $\mathrm{x}=$ 0.54 at $190 \mathrm{~K}$ ) is shown as a dashed black line).
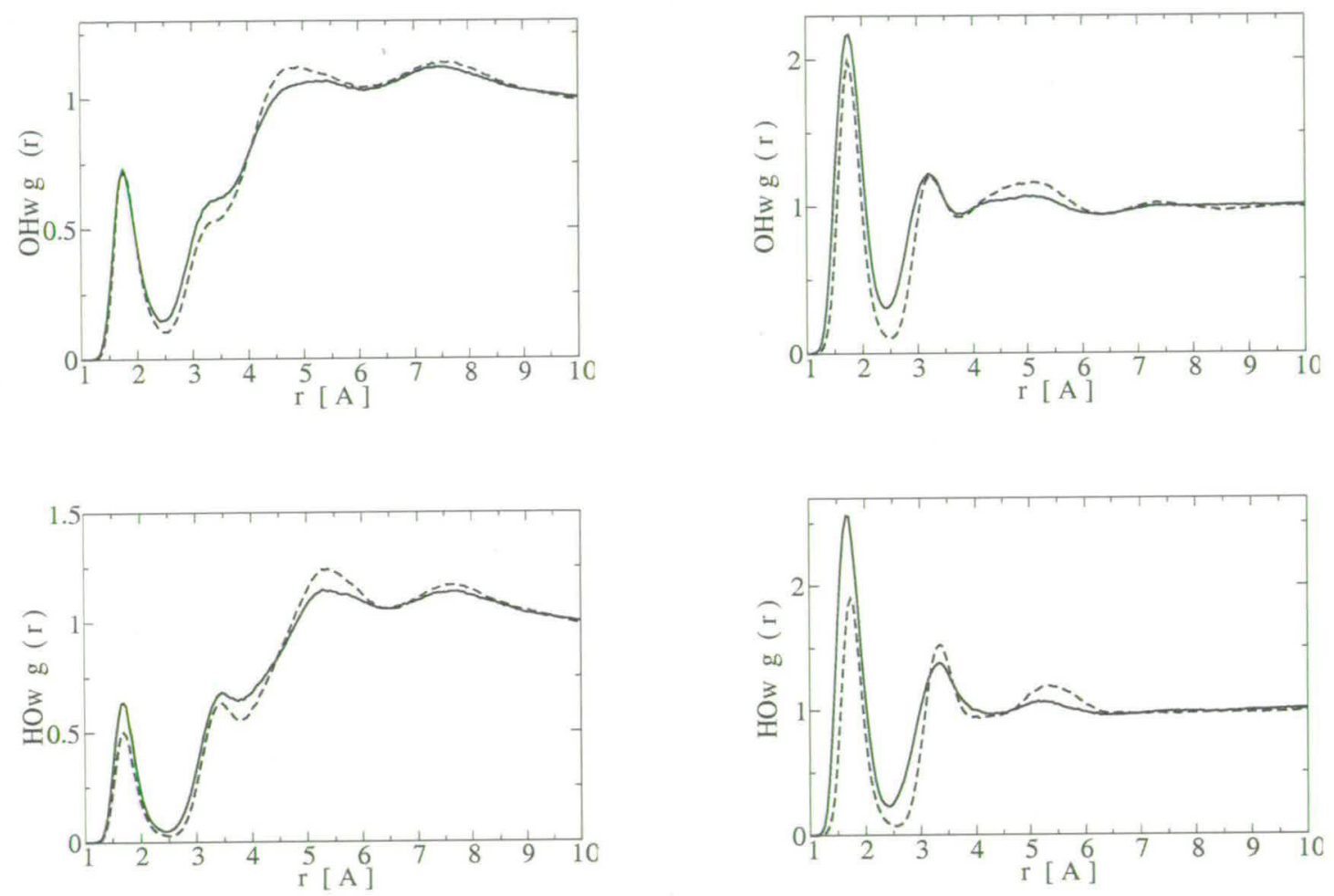

Figure 6.7: Top panel shows partial radial distribution function for $\mathrm{OHw}$ correlations in a methanol-water $x=0.27$ mole fraction mixture (left) at $293 \mathrm{~K}$ (black solid line) and mixture at $238 \mathrm{~K}$ (black dashed line) and for a methanol-water $\mathrm{x}=0.54$ mole fraction mixture (right) at $298 \mathrm{~K}$ (black solid line), mixture at $260 \mathrm{~K}$ (black dashed line) and mixture at $190 \mathrm{~K}$ (black dotted line). Lower panel shows partial radial distribution function for HOw correlations in a methanol-water $x=0.27$ mole fraction mixture (left) at $293 \mathrm{~K}$ (black solid line) and mixture at $238 \mathrm{~K}$ (black dashed line) and for a methanol-water $\mathrm{x}=0.54$ mole fraction mixture (right) at $298 \mathrm{~K}$ (black solid line), mixture at $260 \mathrm{~K}$ (black dashed line) and mixture at $190 \mathrm{~K}$ (black dotted line)

The first peak at around $1.75 \AA$ is due to hydrogen bonds between methanol molecules and water molecules. Integration under each of these peaks results in the first shell coordination number (see Table C.3 for methanol-water $\mathrm{x}=0.27$ mole fraction and Table C. 4 for $\mathrm{x}=0.54$ mole fraction). The coordination numbers show that on average there is a greater probability of a water molecule hydrogen bonding with the oxygen 
of the methanol than with the hydrogen of hydroxyl group of methanol. This trend in the methanol-water hydrogen bonding has also been observed in a more concentrated mixture of methanol-water, $x=0.70$ mole fraction[12].

Next, consider the effect of cooling on the hydrogen bonding between methanol molecules and water molecules. As the $\mathrm{x}=0.27$ system and the $\mathrm{x}=0.54$ system is cooled the coordination numbers for the first shell in the partial radial distribution functions $g_{O H w}(r)$ and $g_{H O w}(r)$ decreases. This reduction in coordination numbers implies that there is less hydrogen bonding between methanol and water when the system is cooled, a further indication of a more segregated system.

Next, consider the correlations between the oxygens of methanol molecules and water molecules. Figure 6.8 shows $g_{0 O w}(r)$ for methanol water $\mathrm{x}=0.27$ mole fraction (left) and $\mathrm{x}=0.54$ mole fraction (right). The room temperature measurement is shown as a solid black line while the cooled system $(x=0.27$ at $238 \mathrm{~K}$ and $\mathrm{x}=0.54$ at $190 \mathrm{~K})$ is shown as a dashed black line.
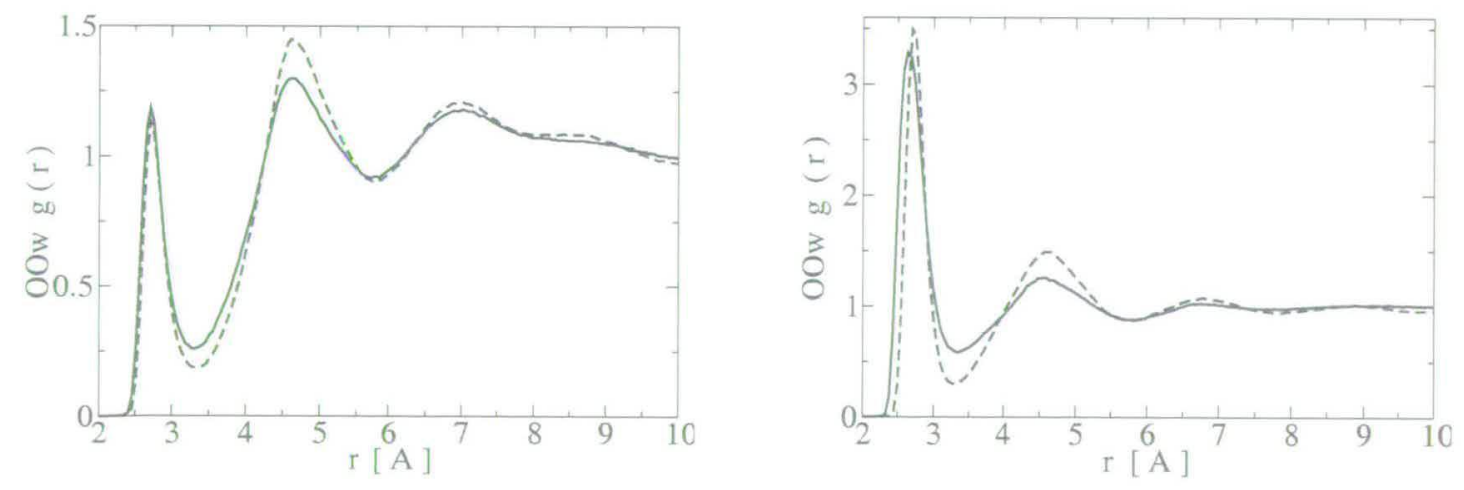

Figure 6.8: Partial radial distribution function for $00 \mathrm{w}$ correlations in a methanol-water $\mathrm{x}=$ 0.27 mole fraction mixture (left) at $293 \mathrm{~K}$ (black solid line) and mixture at $238 \mathrm{~K}$ (black dashed line) and for a methanol-water $x=0.54$ mole fraction mixture (right) at $298 \mathrm{~K}$ (black solid line), mixture at $260 \mathrm{~K}$ (black dashed line) and mixture at $190 \mathrm{~K}$ (black dotted line).

As both systems are cooled the coordination numbers for the first shell decreases. At $\mathrm{x}=0.27$ at $293 \mathrm{~K}$ the coordination number for the first shell of $g_{0 O w}(r)$ is 0.80 , while ar $238 \mathrm{~K}$ this has reduced to 0.64 . At $\mathrm{x}=0.54$ at $298 \mathrm{~K}$ the coordination number for 
the first shell of $g_{O O w}(r)$ is 1.30 while at $190 \mathrm{~K}$ this has reduced to 0.98 . Again, this indicates that there is less hydrogen bonding between methanol molecules and water molecules as the system is cooled. The maximum number of waters that the alcohol hydroxyl group can accommodate is 3 . The second peak is around $4.6 \AA$ indicating that the water molecules are strongly correlated around the hydroxyl group.

Now, consider the hydrogen bonding between methanol molecules and other methanol molecules. At $\mathrm{x}=0.27$ mole fraction and $\mathrm{x}=0.54$ mole fraction there are still some methanol-methanol hydrogen bonds. This can be seen in Figure 6.9 which shows $g_{O H}(r)$ for methanol water $\mathrm{x}=0.27$ mole fraction (left) and $\mathrm{x}=0.54$ mole fraction (right). The room temperature measurement is shown as a solid black line while the cooled system $(x=0.27$ at $238 \mathrm{~K}$ and $\mathrm{x}=0.54$ at $190 \mathrm{~K})$ is shown as a dashed black line. In both RDF's the distribution is also shown for pure methanol (red line) for comparison.
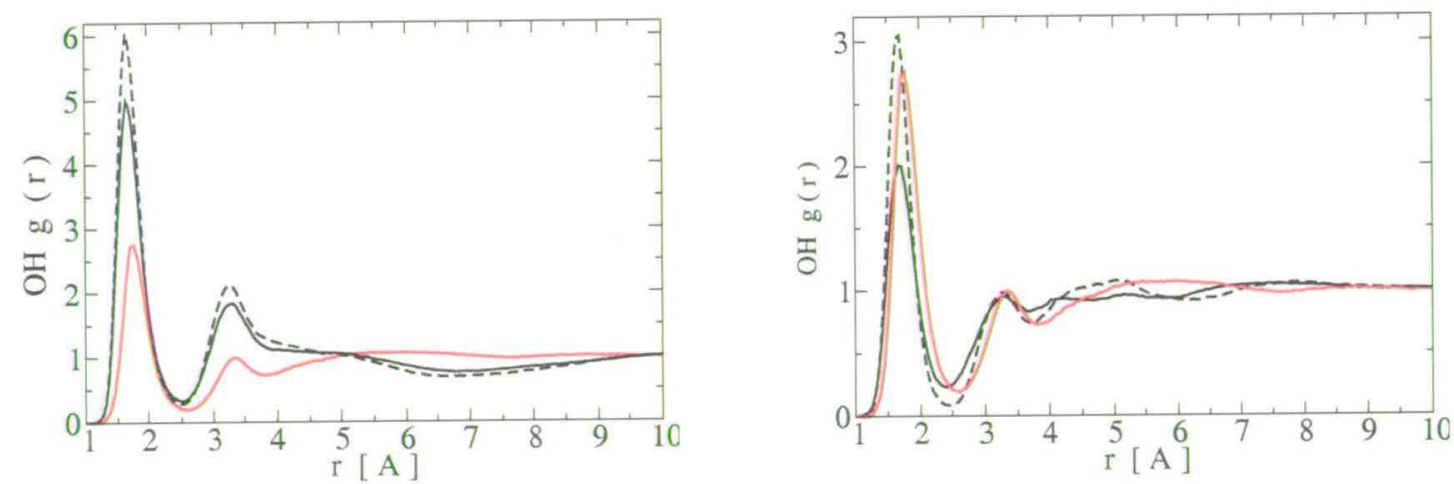

Figure 6.9: Partial radial distribution function for $\mathrm{OH}$ correlations in a methanol-water $\mathrm{x}=0.27$ mole fraction mixture (left) at $293 \mathrm{~K}$ (black solid line) and mixture at $238 \mathrm{~K}$ (black dashed line) and for a methanol-water $\mathrm{x}=0.54$ mole fraction mixture (right) at $298 \mathrm{~K}$ (black solid line), mixture at $260 \mathrm{~K}$ (black dashed line) and mixture at $190 \mathrm{~K}$ (black dotted line) In both $\mathrm{g}(\mathrm{r}) \mathrm{s}$ the distribution is also shown for pure methanol (red line) for comparison.

For pure methanol the coordination number for the first shell in $g_{O H}(r)$ is 0.9 (Table C.1[106]). With the addition of water at the two different concentrations this coordination number reduces to 0.64 for $\mathrm{x}=0.27$ mole fraction and 0.47 for $\mathrm{x}=0.54$ mole fraction. This is to be expected as there is more competition from the water molecules in 
the methanol environment. However, as the systems are cooled the coordination numbers increase to 0.76 for $\mathrm{x}=0.27$ at $238 \mathrm{~K}$ and 0.63 for $\mathrm{x}=0.54$ at $190 \mathrm{~K}$. Therefore, as the methanol water system is cooled on average the probability of methanol-methanol hydrogen bonding increases.

Two other partial distribution plots can also be examined to further explore methanolmethanol hydrogen bonding. Figures 6.10 and 6.11 show the correlations for $g_{O O}(r)$ and $g_{H H}(r)$ respectively for both $\mathrm{x}=0.27$ and $\mathrm{x}=0.54$ mole fraction. The first and second peak in the RDF's for both concentrations are seen to contract to lower $\mathrm{r}$ values compared to the distribution for pure methanol. The methanol in the methanol-water mixture appears to be associating. As the systems are cooled there are some subtle changes in $g_{O O}(r)$. At $\mathrm{x}=0.27$ there is a slight contraction of the 2 nd peak for methanol OO hydrogen bonding. At $x=0.54$ there is a very slight contraction of the 1st peak while the second peak moves to larger r values. Overall, the effect of cooling on the $\mathrm{OO}$ correlations displayed in $g_{O O}(r)$ are very small and are possibly just reflecting a sharpening of the structure in the system.
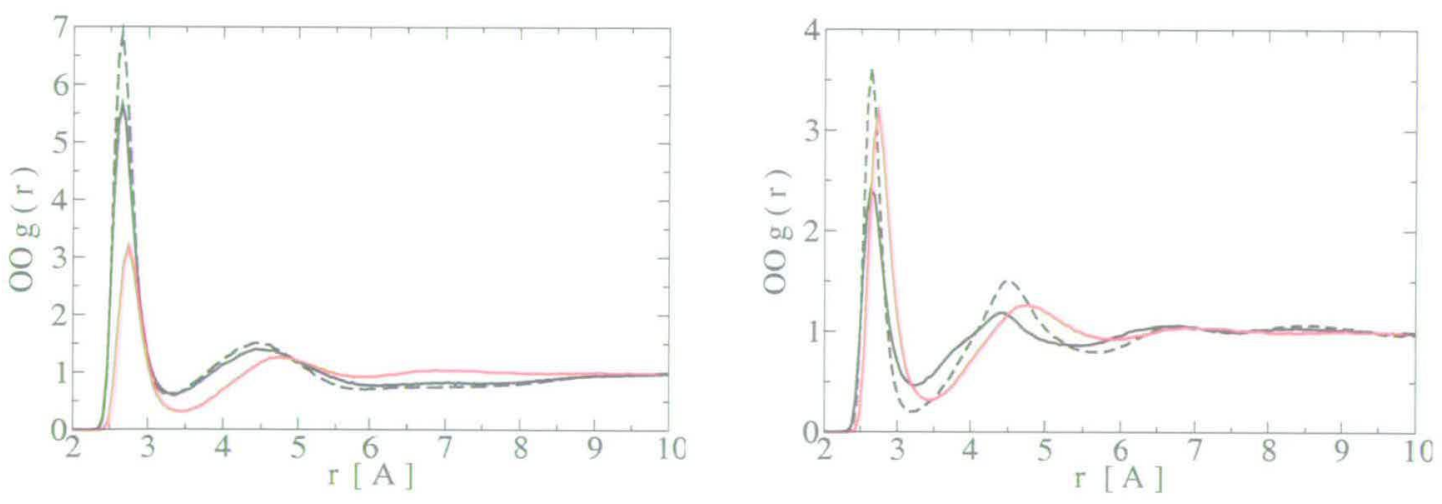

Figure 6.10: Partial radial distribution function for $O O$ correlations in a methanol-water $\mathrm{x}=$ 0.27 mole fraction mixture (left) at $293 \mathrm{~K}$ (black solid line) and mixture at $238 \mathrm{~K}$ (black dashed line) and for a methanol-water $x=0.54$ mole fraction mixture (right) at $298 \mathrm{~K}$ (black solid line), mixture at $260 \mathrm{~K}$ (black dashed line) and mixture at $190 \mathrm{~K}$ (black dotted line) In both RDF's the distribution is also shown for pure water (red line) for comparison.

For pure methanol the coordination shell for the first shell is 1.9 for $g_{O O}(r)$ and 2.1 

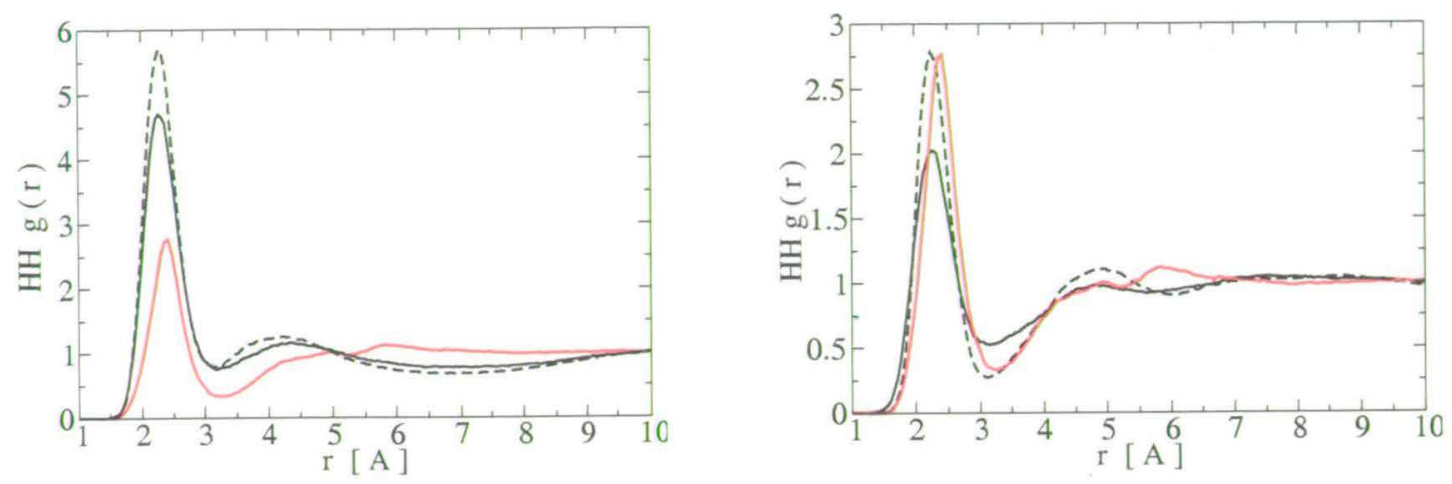

Figure 6.11: Partial radial distribution function for $\mathrm{HH}$ correlations in a methanol-water $\mathrm{x}=$ 0.27 mole fraction mixture (left) at $293 \mathrm{~K}$ (black solid line) and mixture at $238 \mathrm{~K}$ (black dashed line) and for a methanol-water $\mathrm{x}=0.54$ mole fraction mixture (right) at $298 \mathrm{~K}$ (black solid line), mixture at $260 \mathrm{~K}$ (black dashed line) and mixture at $190 \mathrm{~K}$ (black dotted line) In both RDF's the distribution is also shown for pure water (red line) for comparison.

for $g_{H H}(r)$. At $\mathrm{x}=0.27$ mole fraction this reduces to 1.55 for $g_{O O}(r)$ and 1.70 for $g_{H H}(r)$, while at $\mathrm{x}=0.54$ mole fraction this reduces to 1.29 for $g_{O O}(r)$ and 1.33 for $g_{H H}(r)$. The coordination numbers for the first shell in both of these systems at room temperature is less than the coordination number in pure methanol. This is to be expected as water-methanol hydrogen bonding will be competing with methanolmethanol bonding to solvate the alcohol hydroxyl group.

As the methanol-water system is cooled these coordination numbers increase. For $\mathrm{x}=$ 0.27 at a temperature of $238 \mathrm{~K}$ the coordination number increases to 1.79 for $g_{O O}(r)$ and 2.05 for $g_{H H}(r)$, while at $\mathrm{x}=0.54$ mole fraction and $190 \mathrm{~K}$ the coordination number increases to 1.49 for $g_{O O}(r)$ and 1.69 for $g_{H H}(r)$. There are therefore more methanol molecules solvating the methanol hydroxyl group as the temperature of the system is lowered. This would imply a more segregated system for the methanol and water.

From the previous inspection of methanol-water hydrogen bonding it appears that the methanol-methanol hydrogen bonding is increasing at the expense of methanol-water bonding in the cooled systems. If this is true then it might be expected the water-water 
hydrogen bonding to follow the same trend. This will now be explored.

Hydrogen bonding between water molecules and water molecules can be explored by examining $g_{O w H w}(r)$ and $g_{O w O w}(r)$. These RDF's are shown in Figure 6.12 and Figure 6.13 for both $\mathrm{x}=0.27$ and $\mathrm{x}=0.54$ mole fraction.
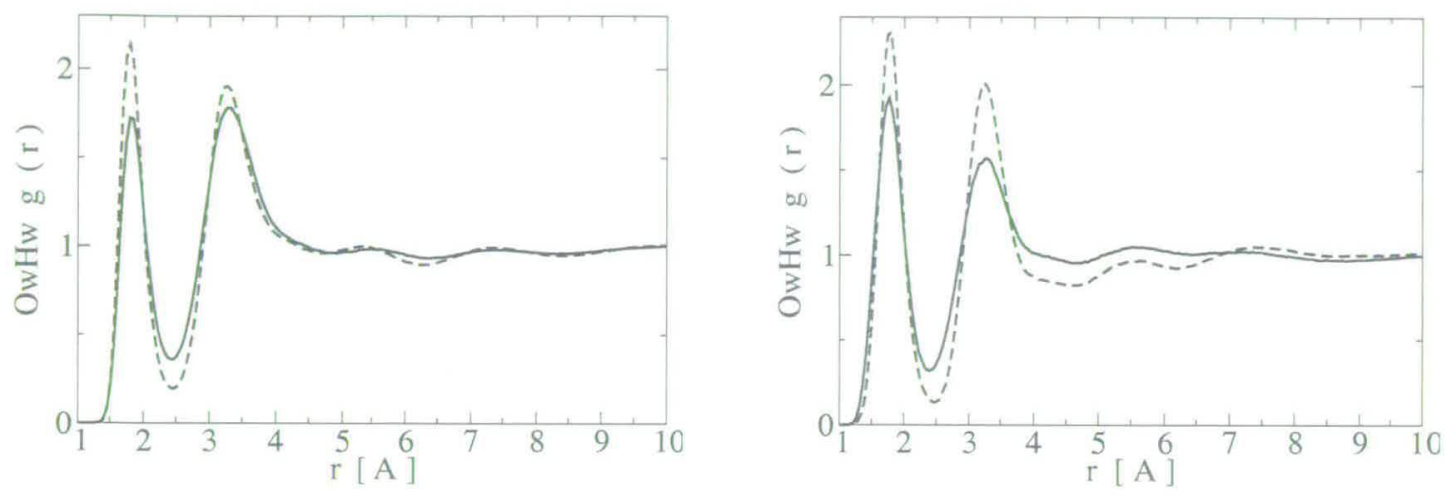

Figure 6.12: Partial radial distribution function for $\mathrm{OwHw}$ correlations in a methanol-water $\mathrm{x}=$ 0.27 mole fraction mixture (left) at $293 \mathrm{~K}$ (black solid line) and mixture at $238 \mathrm{~K}$ (black dashed line) and for a methanol-water $x=0.54$ mole fraction mixture (right) at $298 \mathrm{~K}$ (black solid line), mixture at $260 \mathrm{~K}$ (black dashed line) and mixture at $190 \mathrm{~K}$ (black dotted line) In both RDF's the distribution is also shown for pure water (red line) for comparison.

The radial distribution function for $\mathrm{OwOw}$ correlations in pure water is shown in Figure 6.13. The first peak at $2.7 \AA$ corresponds to the position of the first shell. The position of the second coordination shell at $4.5 \AA$ is taken to be evidence for the extended tetrahedral network of pure water.

At $x=0.27$ the second peak in $g_{O w O w}(r)$ has shifted in to slightly smaller $\mathrm{r}$ (4.44 $\AA$ ) with the addition of methanol. The water molecules are therefore contracting and becoming more preferentially orientated. When the temperature in the $\mathrm{x}=0.27$ system is reduced to $238 \mathrm{~K}$ the second peak in $g_{O w O w}(r)$ sharpens as expected but remains at $4.44 \AA$, i.e. still at smaller $r$ than that of pure water (Figure 6.13).

At $x=0.54$ the second peak in $g_{O w O w}(r)$ shifts out to larger $\mathrm{r}(4.88 \AA)$ on the addition of methanol (Figure 6.13). The methanol in the system (this is a more concentrated mixture) is disrupting the extended water network. As the system is cooled to $190 \mathrm{~K}$ 

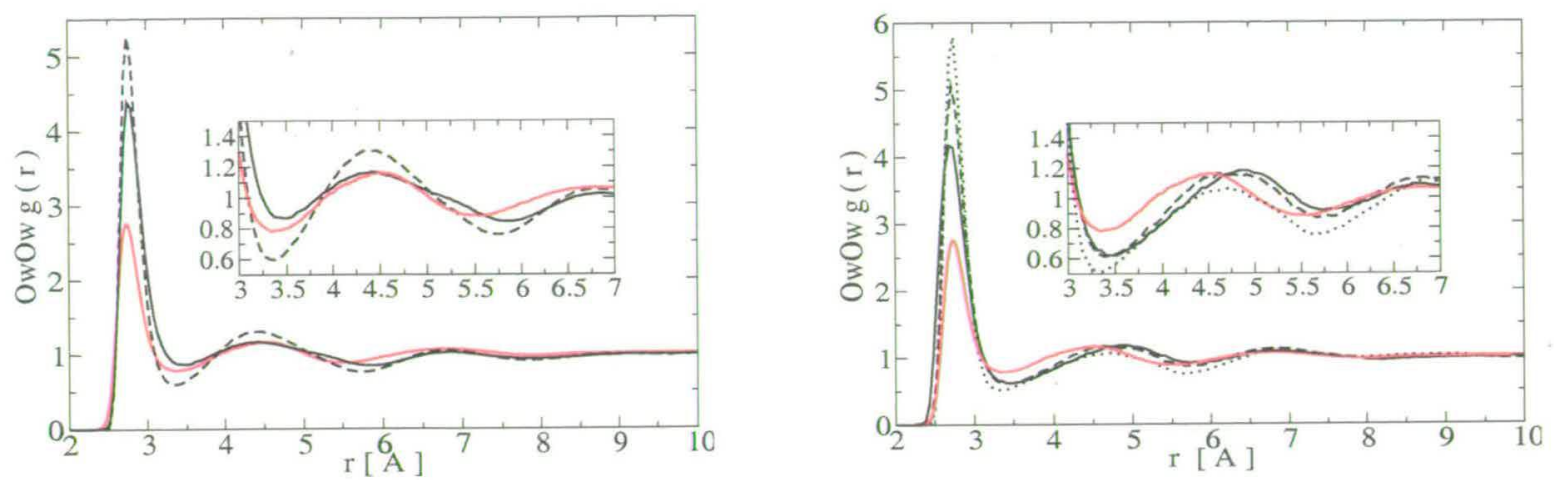

Figure 6.13: Partial radial distribution function for OwOw correlations in a methanol-water $\mathrm{x}=$ 0.27 mole fraction mixture (left) at $293 \mathrm{~K}$ (black solid line) and mixture at $238 \mathrm{~K}$ (black dashed line) and for a methanol-water $\mathrm{x}=0.54$ mole fraction mixture (right) at $298 \mathrm{~K}$ (black solid line), mixture at $260 \mathrm{~K}$ (black dashed line) and mixture at $190 \mathrm{~K}$ (black dotted line) In both RDF's the distribution is also shown for pure water (red line) for comparison.

the peak shifts back in to lower $\mathrm{r}(4.68 \AA)$. It seems that cooling somehow allows water to almost retain a tetrahedral-like network in the system.

The coordination number in the first shell has changed from 4.6 in pure water to 3.59 in $x=0.27$ at $298 \mathrm{~K}$ and 3.67 in $x=0.27$ at $238 \mathrm{~K}$. The coordination number in the second shell has changed from 18.6 in pure water to 12.09 in $x=0.27$ at $298 \mathrm{~K}$ and 12.04 in $x=0.27$ at $238 \mathrm{~K}$. At this concentration, reducing the temperature increases the coordination of water only at 1 st neighbour distance. At $x=0.54$ the coordination number in the first shell has changed from 1.93 at $298 \mathrm{~K}, 1.95$ at $260 \mathrm{~K}$ to 2.06 at 190 $\mathrm{K}$. The coordination number in the second shell has changed from 5.99 at $298 \mathrm{~K}, 5.74$ at $260 \mathrm{~K}$ to 5.13 at $190 \mathrm{~K}$. At this concentration, reducing the temperature increases the coordination only at 1 st neighbour distance.

Therefore, as both systems are cooled the coordination numbers for the first shell in $g_{O w H w}(r)$ and $g_{O w O w}(r)$ increase. Therefore, cooling the system increases the amount of hydrogen bonding between water-water molecules to first neighbour level.

The local water structure can be further explored by considering the distribution of bond angles between neighbouring water molecules. This is done by examining the 


\begin{tabular}{|c|c|c|c|}
\hline $\begin{array}{c}\text { Concentration } \\
x\end{array}$ & $\begin{array}{c}\text { Temp. } \\
/ K\end{array}$ & $\begin{array}{c}\text { angle 1 } \\
/{ }^{\circ}\end{array}$ & $\begin{array}{c}\text { angle 2 } \\
{ }^{\circ}\end{array}$ \\
\hline 0.00 & 298 & 55.30 & 100.70 \\
\hline 0.27 & 293 & 57.33 & 102.80 \\
\hline 0.27 & 238 & 57.77 & 103.80 \\
\hline 0.54 & 298 & 57.10 & 118.46 \\
\hline 0.54 & 260 & 56.43 & 110.63 \\
\hline 0.54 & 190 & 55.53 & 109.23 \\
\hline
\end{tabular}

Table 6.2: Triplet bond angle distributions for $O_{W} O_{W} O_{W}$ for pure water, methanol-water $x=$ 0.27 and $x=0.54$ at a number of different temperatures after 10000 iterations

triplet bond angle distributions (illustrated in inset of Figure 6.14). This distribution is obtained by calculating all of the included angles between triplets of oxygen atoms $O_{w}$ in water. A program can be used to calculate the distribution of included angles of a triplet of atoms separated by specified distances determined from $g_{O w O w}$. Atoms are specified in triplets and the angle calculated is the included angle formed by the middle atom of the triplet (Figure 6.14).

Figure 6.14 shows the triplet bond angle distribution for OwOwOw, three oxygens of water molecules, for methanol-water $\mathrm{x}=0.27$ (left) and $\mathrm{x}=0.54$ (right) at both room temperature and under cooling. The triplet bond angle distribution for pure water is also shown for comparison (red line). Cooling the methanol-water system increases the frequency of the triplet bond angle distribution, i.e peak heights increase as the mixture is cooled. This is expected as the liquid becomes more structured. At $x=0.54$ mole fraction there is a very clear change in the peak position of the bond angle. As this mixture is cooled the structure of the water is clearly becoming more tetrahedrallike, moving to $110^{\circ}$ (very close to the tetrahedral value of $109.5^{\circ}$ which is illustrated on Figure 6.14 by a vertical blue dashed line). 

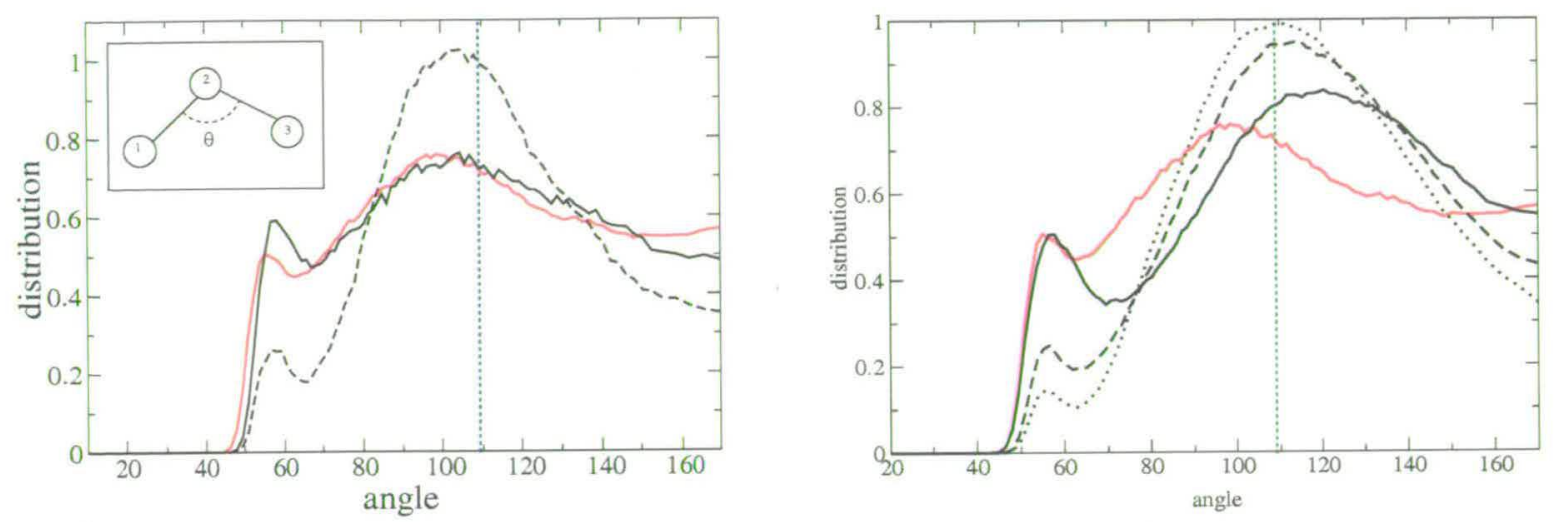

Figure 6.14: The triplet bond angle distribution for $O_{W}-O_{W}-O_{W}$ is shown on the left for methanol-water $x=0.27$ at $293 \mathrm{~K}$ (solid black line) and 238K (dashed black line) and on the right for methanol-water $x=0.54$ at $298 \mathrm{~K}$ (solid black line), 260K (dashed black line) and $190 \mathrm{~K}$ (dotted black line). The triplet bond angle distribution for $O_{W}-O_{W}-O_{W}$ for pure water at $298 \mathrm{~K}$ is also shown on both graphs(solid red line). The vertical line illustrates the position of the tetrahedral angle $109.5^{\circ}$

Therefore, the overall picture is clear. As the methanol-water system is cooled at $\mathrm{x}$ $=0.27$ and $x=0.54$ mole fraction the hydrogen bonding interactions change significantly. At room temperature there is a network of hydrogen bonds which are mixed, with interactions between methanol molecules, water molecules as well as between methanol and water molecules. When these systems are cooled the hydrogen bonding interactions change. The hydrogen bonding interactions between like molecules (methanol-methanol and water-water) is enhanced at the expense of hydrogen bonding interactions between unlike molecules (methanol-water). This local structure information provides further evidence that there is enhanced micro-segregation on cooling. 


\subsection{Cooling Enhances Hydrophobic Interaction}

Consider how the presence of water effects the non-polar interactions of methanol molecules. The hydrophobic effect is thought to drive non-polar species to associate and therefore expel water from their hydration layer. Therefore, it is essential to understand the behaviour of the non-polar species in these solutions. Since the carbon atom has been used to define the methanol molecular centre, $g_{C C}(r)$ can provide information on inter-methanol interactions. Figure 6.15 shows the radial distribution function $g_{C C}(r)$ for methanol-methanol correlations for the two systems under investigation. On the left, $\mathrm{x}=0.27$ mole fraction at $293 \mathrm{~K}$ (solid black line) and $238 \mathrm{~K}$ (dashed black line) and on the right $\mathrm{x}=0.54$ mole fraction at $298 \mathrm{~K}$ (solid black line), $260 \mathrm{~K}$ (dashed black line) and $190 \mathrm{~K}$ (dotted black line). The $g_{C C}(r)$ for pure methanol (solid red line) is also shown for comparison[106].
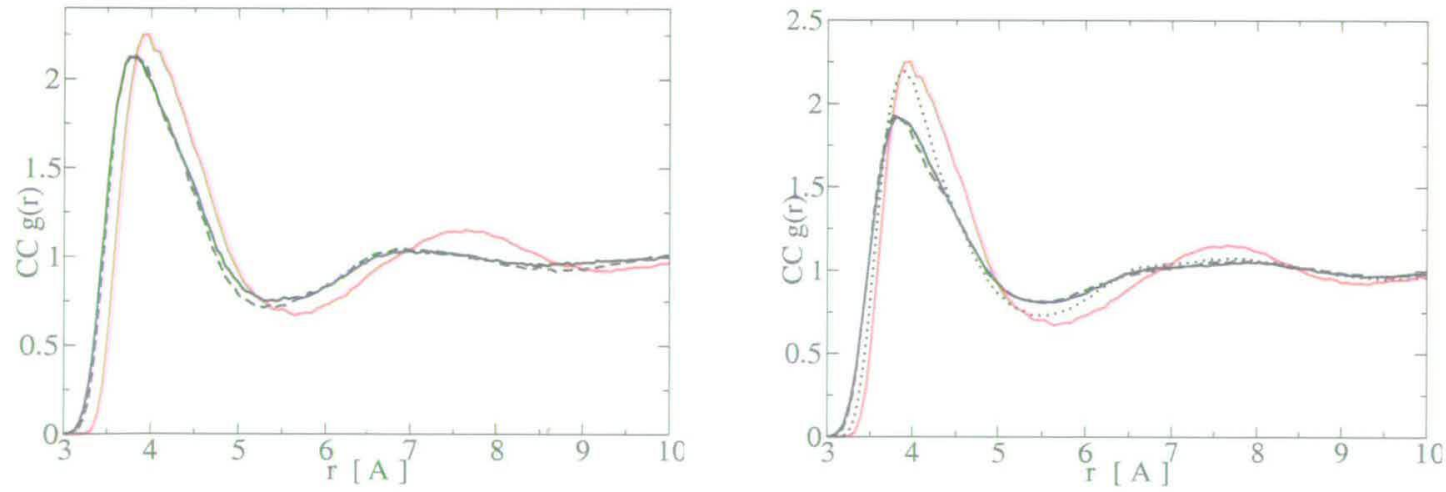

Figure 6.15: Partial radial distribution function for CC correlations in a methanol-water $\mathrm{x}=$ 0.27 mole fraction mixture (left) at $298 \mathrm{~K}$ (black solid line) and mixture at $238 \mathrm{~K}$ (black dashed line) and for a methanol-water $x=0.54$ mole fraction mixture (right) at $298 \mathrm{~K}$ (black solid line), mixture at $260 \mathrm{~K}$ (black dashed line) and mixture at $190 \mathrm{~K}$ (black dotted line). Pure methanol at $298 \mathrm{~K}$ is also shown for comparison(red line).

A distinct and broad first coordination shell is observed indicating solute molecular contacts centred on this distance. At room temperature, $x=0.27$ the methanolmethanol CC contact distance has contracted to lower $\mathrm{r}, \sim 3.77 \AA$, compared to a distance of $\sim 3.90 \AA$ in pure methanol. This contraction of $g_{C C}(r)$ has also been ob- 
served in a more concentrated methanol-water solution $x=0.70$ mole fraction and is believed to be illustrative of an enhancement of the non-polar groups[12]. In the presence of water the methanol methyl groups are packing closer together and therefore the contact distances (seen in $g_{C C}(r)$ ) are decreasing. Reducing the temperature of the $x=0.27$ mole fraction mixture does not appear to have any significant effect on the distribution function $g_{C C}$. At room temperature, $x=0.54$ the $\mathrm{CC}$ contact distance also contracts to lower $r$ values, $\sim 3.80 \AA$ (Figure 6.15 (right)) but when the temperature of the system is lowered from $298 \mathrm{~K}$ to $190 \mathrm{~K}$ this peak shifts back out slightly to $3.88 \AA$. However, the effect is not big. The coordination numbers for the 1 st shell changes from 10.8 in pure methanol to 4.45 in $x=0.27$ at $298 \mathrm{~K}$ and 4.34 in $x=0.27$ at $238 \mathrm{~K}$. i.e. at this concentration as the temperature is reduced the coordination number for methanol decreases. The coordination numbers for the $x=0.54$ mixture have changed from 7.21 at $298 \mathrm{~K}, 7.36$ at $260 \mathrm{~K}$ and 7.38 at $190 \mathrm{~K}$. At this concentration, as the temperature reduces the coordination number for methanol increases.

Therefore, the overall picture is that there are very subtle differences in the behaviour of the methanol-methanol interactions at the two different concentrations under investigation. At $x=0.27$ there is a contraction of the methyl group contact distance which is not changed on cooling. In the slightly more concentrated system, $x=0.54$ the methyl group contact distance also contracts but this effect is reversed (if only by small amount) when the system is cooled. This is also illustrated in the coordination numbers which increase slightly on cooling. Therefore, non-polar group association in terms of CC contact distances shows no dramatic indications of a change in solute association with cooling. For the two systems studied, at these particular state points, the hydrophobic interaction does not play a strong role for these intermolecular contacts. Perhaps it is elsewhere that evidence can be found for enhanced solute association.

Figure 6.16 shows the radial distribution function $g_{C O}(r)$ for methanol-methanol correlations for the two systems under investigation. On the left, $\mathrm{x}=0.27$ mole fraction at $293 \mathrm{~K}$ (solid black line) and $238 \mathrm{~K}$ (dashed black line) and on the right $\mathrm{x}=0.54$ mole 
fraction at $298 \mathrm{~K}$ (solid black line), $260 \mathrm{~K}$ (dashed black line) and $190 \mathrm{~K}$ (dotted black line). $g_{C C}(r)$ for pure methanol (solid red line) is also shown for comparison[106].
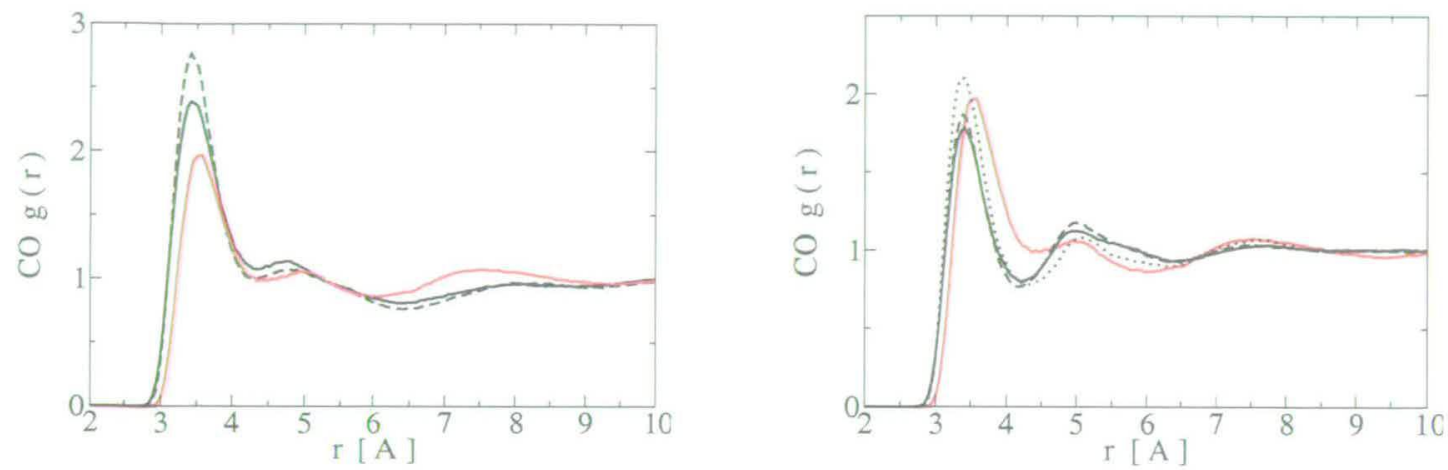

Figure 6.16: Partial radial distribution function for $\mathrm{CO}$ correlations in a methanol-water $\mathrm{x}=0.27$ mole fraction mixture (left) at 298K (black solid line) and mixture at 238K (black dashed line) and for a methanol-water $\mathrm{x}=0.54$ mole fraction mixture (right) at $298 \mathrm{~K}$ (black solid line), mixture at $260 \mathrm{~K}$ (black dashed line) and mixture at 190K (black dotted line). Pure methanol at 298K is also shown for comparison(red line).

The first peak of $g_{C O}(r)$ can be attributed to inter molecular contacts of two interacting methanol molecules via the hydroxyl group while the second peak comes from interactions via the methyl end of a methanol molecule. In Figure 6.16 the first peak in $g_{C O}(r)$ is observed to contract at both $\mathrm{x}=0.27$ and $\mathrm{x}=0.54$ mole fraction. The increased association between the methanol molecules at room temperature noted from the carbon-carbon correlations is also observed in the carbon-oxygen correlations. The preferential interaction is enhanced as these systems are cooled, as seen by the further contraction of the first peak in $g_{C O}(r)$. The coordination numbers of the first shell in $g_{C O}(r)$ increase as the methanol-water system is cooled (Tables C.3 and C.4). Figure 6.17 shows the radial distribution function $g_{C M}(r)$ for methanol-methanol correlations between carbon and the hydrogen of the methanol methyl group for the two systems under investigation. Again, compared to pure methanol, the first shoulder of $g_{C M}(r)$ moves to a lower $\mathrm{r}$ value at both $\mathrm{x}=0.27$ and $\mathrm{x}=0.54$ mole fraction. In the more concentrated system $(x=0.54)$ the distribution is much better defined in the mixture. Adding 45 mole percent of water to methanol results in an enhancement of the contact 
between the non-polar groups of methanol. There appears to be an enhancement in the packing of the methyl groups induced by adding water. When these systems are cooled the distributions become even better defined but no further contraction is observed.
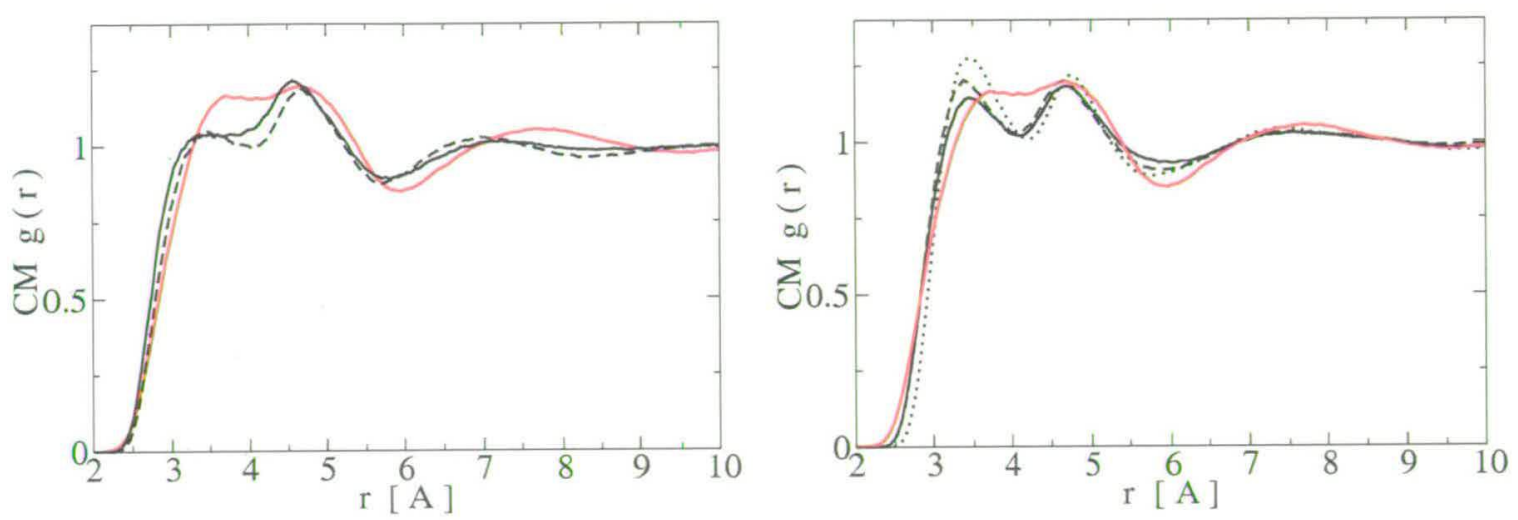

Figure 6.17: Partial radial distribution function for $\mathrm{CM}$ correlations in a methanol-water $\mathrm{x}=0.27$ mole fraction mixture (left) at $298 \mathrm{~K}$ (black solid line) and mixture at $238 \mathrm{~K}$ (black dashed line) and for a methanol-water $\mathrm{x}=0.54$ mole fraction mixture (right) at $298 \mathrm{~K}$ (black solid line), mixture at $260 \mathrm{~K}$ (black dashed line) and mixture at $190 \mathrm{~K}$ (black dotted line). Pure methanol at $298 \mathrm{~K}$ is also shown for comparison(red line). 


\subsection{Methanol-Water Correlations}

In a mixture of methanol and water there are micro-segregated regions of water and of methanol. However, there will also be an interface between the methanol and water where the non-polar species of methanol will be in the vicinity of water molecules. Consider now how this interface changes at different concentrations under cooling. If the interface is changing then the entropy or disorder of the system must also be changing and thus the thermodynamics will be different.

To learn about the organisation of water molecules in the neighbourhood of a methanol molecule the partial radial distribution function $g_{C O w}(r)$ can be examined which gives the correlation between carbon in methanol and oxygen in water. Figure 6.18 shows $g_{C O w}(r)$ for $\mathrm{x}=0.27$ mole fraction system (left) and $g_{C O w}(r)$ for $\mathrm{x}=0.54$ mole fraction system (right). The well defined peak at $\sim 3.45 \AA$ for the $\mathrm{x}=0.27$ system has shifted to $\sim 3.40 \AA$ for the $\mathrm{x}=0.54$ system. In previous studies of a more concentrated solution, $\mathrm{x}=0.70$, this peak was observed at $\sim 3.30 \AA$, while for a more dilute solution, $\mathrm{x}=$ 0.05 the peak was observed at $\sim 3.61 \AA$. Therefore, the overall picture seems to be that as more water is added to the methanol the first peak in $g_{C O w}(r)$ moves to larger values of $\mathrm{r}$.

At $x=0.27$ the first peak in $g_{C O w}(r)$ shifts outwards slightly to larger $\mathrm{r}$ as the temperature is decreased from $293 \mathrm{~K}$ to $238 \mathrm{~K}$ (Figure 6.18 (left)). This is also the case at $x=0.54$, as the temperature is reduced from $298 \mathrm{~K}$ to $190 \mathrm{~K}$ the first peak $g_{C O w}(r)$ increases slightly to larger r, changing from $3.36 \AA$ to $3.47 \AA$ (Figure 6.18 (right)). Therefore, as the methanol-water solution is cooled the carbon of methanol and oxygen of water are becoming more widely separated. This observation is consistent with the picture of an enhancement between like-like species on cooling. As the temperature is lowered methanol is becoming more segregated from the water and thus the microheterogeneity is increasing. This enhancement is also observed by considering the coordination numbers for both methanol-water systems. For the methanol-water mole 
fraction $x=0.27$ mole fraction system, increases in the coordination number with cooling were observed for the majority of like-like correlations. There was a decrease in the coordination number for correlations between methanol and water species, in particular for for hydrogen bonding correlations. Therefore it seems that the effect of cooling the $x=0.27$ mixture is to enhance correlations between most methanolmethanol contacts and water-water contacts at the expense of inter methanol-water hydrogen bonding correlations. This trend was also observed in the methanol-water $x=0.54$ mole fraction system.
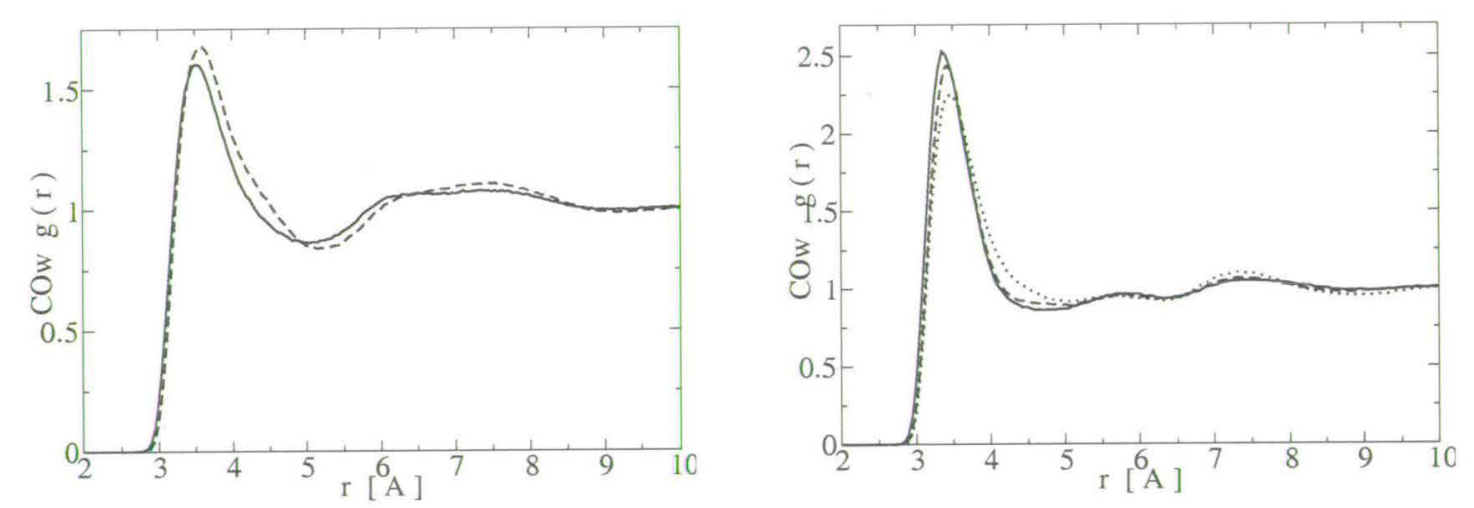

Figure 6.18: Partial radial distribution function for COw correlations in a methanol-water $\mathrm{x}=$ 0.27 mole fraction mixture (left) at $293 \mathrm{~K}$ (black solid line) and mixture at $238 \mathrm{~K}$ (black dashed line) and for a methanol-water $\mathrm{x}=0.54$ mole fraction mixture (right) at $298 \mathrm{~K}$ (black solid line), mixture at $260 \mathrm{~K}$ (black dashed line) and mixture at $190 \mathrm{~K}$ (black dotted line).

Therefore, from investigation of the local structure there are a number of strong indications that micro-segregation in the methanol-water system, is enhanced on cooling. The evidence included; enhanced hydrogen bonding interactions between methanolmethanol molecules and water-water molecules, a reduction in inter-molecule hydrogen bonding interactions and an enhancement of association for some non-polar group correlations. All of this evidence is consistent with the result from cluster distribution analysis where it was found that the frequency of percolating water clusters was enhanced on cooling. 


\subsection{Discussion}

A series of methanol-water solutions have been investigated by neutron diffraction over a range of concentrations and temperatures. The diffraction data were analysed using the EPSR technique. A general conclusion of these studies is that lowering the temperature has the effect of enhancing the degree of micro-segregation between methanol and water that occurs in these systems.

This conclusion of enhanced micro-segregation is drawn from the following experimental evidence:

- More frequent existence of larger water clusters in comparison to room temperature data.

- An enhancement of intra-species hydrogen bonding interactions

- A reduction in inter-species hydrogen bonding interactions

- An enhancement of methanol non-polar interactions correlations.

- Water network becoming more extended and structured, approaching tetrahedrallike network

This is the kind of behaviour that would be expected on a microscopic scale if the system were moving toward a phase boundary, characterised by an upper critical solution temperature. Partial miscibility is a common feature of binary liquid phase equilibria in which a mixture separates into two phases of different compositions[107] depending on temperature and pressure. Phase separation is a consequence of a system minimising its free energy. Typically, an immiscible region terminates at an upper critical solution temperature (UCST), above which the mixture is fully miscible. In some hydrogen-bonded systems, however, further cooling leads to re-entrant miscibility and a closed-loop gap in the phase diagram appears $[108,109,110]$. Such immiscibility has 
not been observed in the methanol-water system. This may be because the intervening solid phase precludes access to any possible two-fluid region in methanol-water mixtures. The clustering behaviour at low temperature provides a consistent framework within which to interpret the observed variations in local structure, particularly $g_{\text {OwOw }}(r)$. As the temperature is lowered the formation of larger clusters leads to increased connectivity of the water domains. Within these growing water clusters the local structure evolves toward that of bulk water. The effect is most obvious with the methanol-rich solutions studied, $x=0.54$ mole fraction.

Consider how these observations compare with other work on aqueous systems. Neutron diffraction experiments were carried out on a tertiary butanol-water system (TBAwater) $x=0.06$ mole fraction solution at $298 \mathrm{~K}$ and $338 \mathrm{~K}$ [98]. It was found that the second shell in the water radial distribution function gowOw was 'pulled in' toward the central molecule when TBA was added to water. This provided clear evidence that the presence of TBA increases the structural order in the system at this concentration. This ordering was enhanced with the rise in temperature to $338 \mathrm{~K}$. This was paralled with an increase in the hydrophobic interaction seen in the non-polar to non-polar contacts radial distribution functions $g_{C C}$.

Therefore in the TBA-water system, a larger amphiphilic system, the hydrophobic interaction was enhanced by increasing the temperature. In contrast, the methanolwater system, a much smaller amphiphile system, the hydrophobic interaction was enhanced with decreasing temperature (seen in inward shift of 1 st peak in $g_{C O}$ and $g_{C M}$ for $x=0.54$ at $190 \mathrm{~K}$ compared to at $298 \mathrm{~K}$ ). The water structure also enhanced on cooling at the 2 nd neighbour level in $g_{O w O w}$. It seems that the difference in the size of the amphiphilic molecule and the difference between a dilute system (TBA-water) and more concentrated system (methanol-water) leads to subtleties in the positions of the peaks in the relevant radial distribution functions. Therefore it is essential that other analysis are combined with the RDF's in determining the behaviour of the structure in these systems. It is not enough to simply consider the positions of peaks in RDF's. 
Distributions of triplet bond angles, snapshots of the systems and cluster distributions are important in providing an insight into these complex systems.

Molecular dynamics simulations on a methane water system $x=0.03$ mole fraction has also explored the effects of temperature on the hydrophobic interaction[111]. This work found that at the lowest temperature of $270 \mathrm{~K}$ the non-polar methane particles repelled each other weakly. At this temperature the water in the solution appeared more highly structured than in the bulk and was energetically stable. The hydrophobic solute-solute interactions only became attractive above $300 \mathrm{~K}$, and reached a maximum at $\sim 340 \mathrm{~K}$. When the temperature was raised above $376 \mathrm{~K}$ disaggregation among solute molecules was observed. It was proposed that water molecules in the system preferred to be in the hydration shell of the non-polar molecule at lower temperatures, but in the bulk at higher temperatures.

Although this system is a non-polar species in water rather than an amphiphile in water, the experiments were carried out at some low temperatures making it an important system to compare with the methanol-water results. The weaker non-polar interactions at low temperature are also observed in the methanol-water $x=0.54$ system, observed in the outward shift of the first peak in $g_{C C}$ at $190 \mathrm{~K}$ compared to $298 \mathrm{~K}$. Therefore, lowering the temperature appears to weaken solute association slightly. However, solute association was enhanced on cooling through $g_{C O}$ and $g_{C M}$ correlations. Lowering the temperature resulted in a more ordered tetrahedral water distribution in terms of the 2nd peak in $g_{O w O w}$.

Overall the methanol-water system has proved itself to be a rich source of phenomena which may be of relevance to situations involving much larger and more complicated molecules. Methanol and water are ideally suited to the experimental diffraction and atomistic simulation methodologies due to their simple molecular forms, and their ready availability in different isotopic forms. Yet this simple model system can apparently capture much of the essence of hydrophobicity in aqueous systems in a way that 
it might occur in the much larger molecular entities, with mixed hydrophobic and hydrophilic headgroups of real biological systems. The present results should therefore help guide the search for possible mechanisms which control molecular conformation in aqueous solution. 


\section{Chapter 7}

\section{Compression Enhances}

\section{Segregation}

\subsection{Motivation}

At high temperatures and pressures it is known that the thermophysical properties of water differ greatly from those at ambient conditions and these differences have direct impact on the properties of aqueous solutions. This chapter explores the effects of compression on the structure and micro-segregation of methanol-water mixtures. Two different systems were explored, methanol-water $x=0.50$ mole fraction at $200 \mathrm{~K}$ and ambient pressure ( $1 \mathrm{bar}$ ) and methanol-water $x=0.50$ mole fraction at $200 \mathrm{~K}$ and high pressure ( $2 \mathrm{kbar}$ ). This work allows exploration of the effects of compression on local water structure and the water network and could provide an indication of the relevance of pressure denaturation on more complex biological systems. 


\subsection{Experimental Details}

Isotopically substituted, but otherwise similar mixtures of methanol and water were prepared with the correct methanol-water molecular ratio. A total of 5 samples were measured for methanol-water $x=0.50$ mole fraction, these were:

1. $C D_{3} O D$ in $D_{2} O$

2. $\mathrm{CD}_{3} \mathrm{OH}$ in $\mathrm{H}_{2} \mathrm{O}$

3. A $50: 50$ mixture of 1 and 2

4. $\mathrm{CH}_{3} \mathrm{OD}$ in $\mathrm{D}_{2} \mathrm{O}$

5. A 50:50 mixture of 1 and 4

Isotope substitution on the hydroxyl hydrogens of water and methanol in samples 1, 2, and 3 gave the correlations between hydroxyl hydrogens.

In order to learn more about methanol-methanol interactions, the solute-solute partial structure factor was obtained by isotope substitution on the methyl hydrogens using sample 1,4 , and 5 .

Samples were placed in a high pressure rig system made from a $\mathrm{Ti}-\mathrm{Zr}$ alloy that gives negligible coherent scattering. The experimental apparatus used has been described in detail in Chapter 4. Neutron diffraction measurements on a methanol-water solution mole fraction $x=0.50$ were made at two state points; at $200 \mathrm{~K}$ and ambient pressure ( $1 \mathrm{bar}$ ), and at $200 \mathrm{~K}$ and $2 \mathrm{kbar}$. As before, corrections for attenuation and multiple scattering were made using the ATLAS program suite described in Chapter 4.

These procedures lead to a structure factor $F(Q)$ having the form $F\left(S_{\mathrm{HH}}(Q), S_{\mathrm{XH}}(Q)\right.$, $S_{\mathrm{XX}}(Q)$ ) where $\mathrm{S}_{H H}(\mathrm{Q})$ gives correlations between labelled atoms and $\mathrm{S}_{X H}(\mathrm{Q})$ and 
$\mathrm{S}_{X X}(\mathrm{Q})$ are the two composite partial structure factors which give the remaining correlations between other types of atoms (X) and the labelled atom type $(\mathrm{H})$ in the form of a weighted sum of individual site-site partial structure factors. The intermolecular weights for the composite partial structure factors obtained from the neutron diffraction experiment are given in Appendix B.

All the neutron diffraction experiments that have been described were carried out by the author of this thesis. All of the modelling and analysis was carried out using the Empirical Potential Structural Refinement procedure. 


\begin{tabular}{|c|c|c|c|c|c|c|}
\hline $\begin{array}{c}\text { Mole frac. } \\
x\end{array}$ & $\begin{array}{c}\text { Temp. } \\
/ K\end{array}$ & $\begin{array}{c}\text { Pressure } \\
\text { kbar }\end{array}$ & $\begin{array}{c}\text { No. Methanol } \\
\text { molecules }\end{array}$ & $\begin{array}{c}\text { No. Water } \\
\text { molecules }\end{array}$ & $\begin{array}{c}\text { No. density } \\
/ \text { atoms } / \AA^{3}\end{array}$ & $\begin{array}{c}\text { Box Size } \\
/ \AA\end{array}$ \\
\hline 0.50 & 200 & 0.001 & 300 & 300 & 0.1026 & 29.74 \\
\hline 0.50 & 200 & 2 & 300 & 300 & 0.1158 & 28.57 \\
\hline
\end{tabular}

Table 7.1: Parameters of the methanol-water mixtures used in the Empirical Potential Structural Refinement.

\subsection{Structure Factor Fits}

EPSR produces an ensemble of three-dimensional molecular configurations of the mixture that are consistent with the available diffraction data. As before a computer model was set-up using known constraints about the system such as its density and molecular geometry of the molecules. The values for the density and box size used in the EPSR refinement are shown in Table 7.1. A comparison between the experimentally measured partial structure factors (black circles) and those generated from the ensemble averaged EPSR procedure (red line) with 10000 configurations are shown in Figure 7.1. The upper figure is for the system methanol-water $x=0.50$ at $200 \mathrm{~K}$ and 1 bar, while the lower figure is for the system methanol-water $x=0.50$ at $200 \mathrm{~K}$ and 2 kbar.

\subsection{Compression Enhances Micro-segregation}

Methanol and water form a micro-segregated solution on mixing. Consider how this segregation is influenced by the introduction of pressure to the system. The dependence of cluster distributions on compression was investigated for two different solutions of methanol-water. Firstly, methanol mole fraction $x=0.50$ at $200 \mathrm{~K}$ and 1 bar, and methanol mole fraction $x=0.50$ at $200 \mathrm{~K}$ and 2 kbar. For water molecules the hydrogen-bond definition was used to designate which molecules belong to a given water cluster. The criterion used is that two water molecules are said to be hydrogen- 

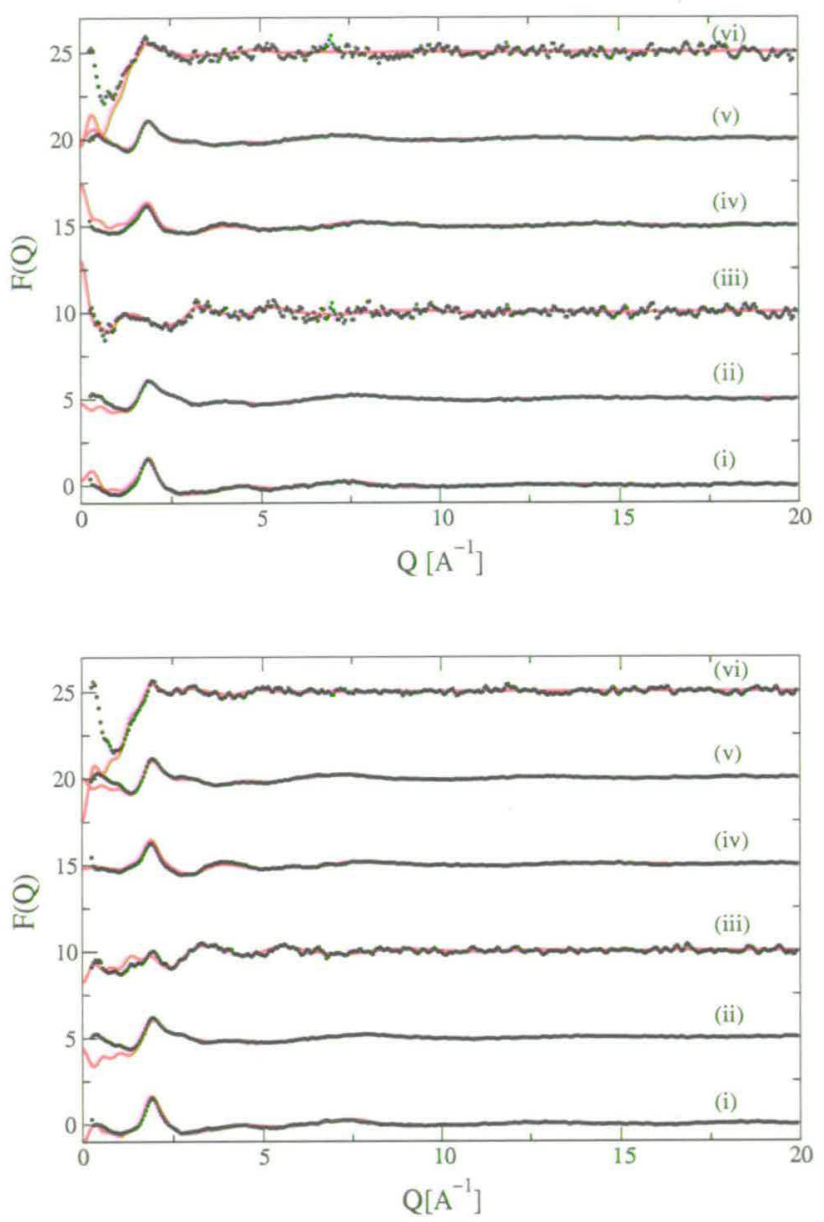

Figure 7.1: Composite partial structure factors fits (red lines) obtained by the EPSR computer simulation procedure compared to the original data (black circles) for methanol-water mixture $x=0.50$ mole fraction at $200 \mathrm{~K}$ and ambient pressure (upper) and $\mathrm{x}=0.50$ mole fraction at $200 \mathrm{~K}$ and $2 \mathrm{kbar}$ (lower).

bonded if their constituent oxygen atoms are less than $g_{O_{W} O_{W}}$ apart, where $g_{O_{W} O_{W}}$ is determined as the minimum following the first peak in the water oxygen-water oxygen pair correlation function (approximately $3.5 \AA$ for EPSR-fitted functions).

For methanol clusters the $g_{C C}$ distance definition was used where two methanol molecules are assigned to the same cluster if the $g_{C C}$ distance is less than the minimum following the first peak determined from the methanol carbon-methanol carbon pair correlation function (which is approximately $5.7 \AA$ ). The methanol cluster size distributions as 

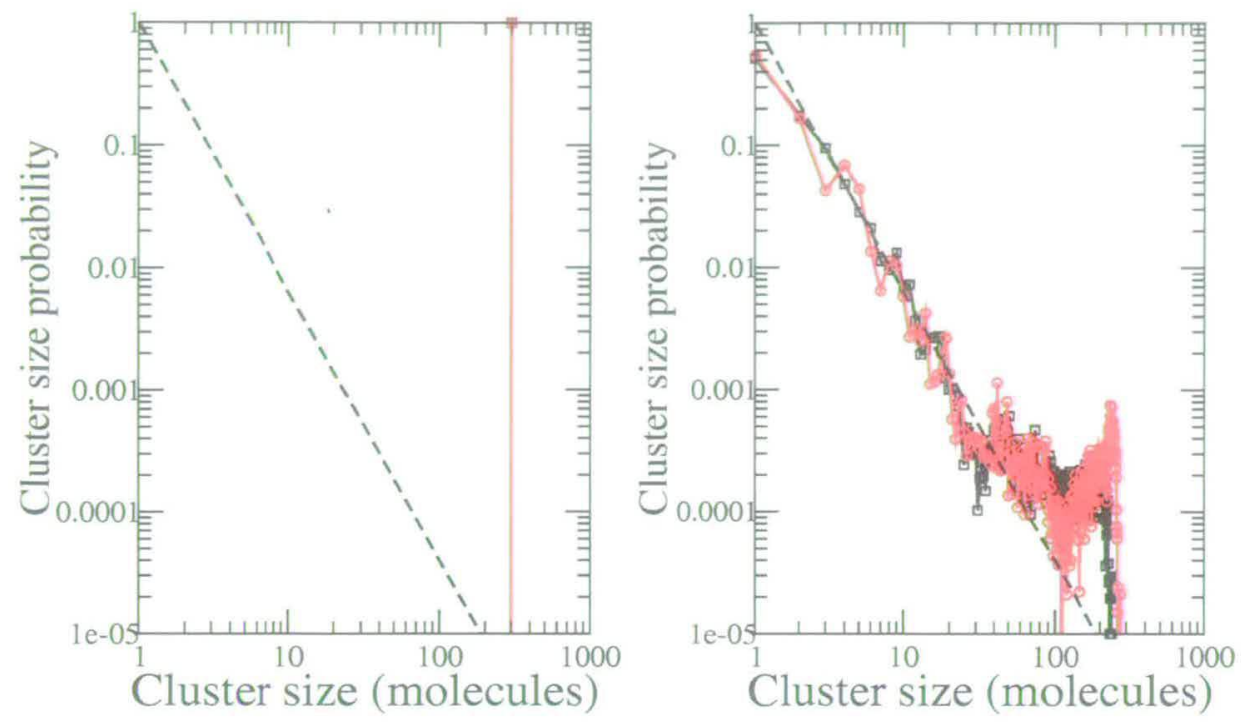

Figure 7.2: Cluster distribution plots for methanol-water $x=0.50$ mole fraction mixture. Graph shows mixture at $200 \mathrm{~K}$ and ambient pressure (black line) and mixture at $200 \mathrm{~K}$ and $2 \mathrm{kbar}$ (red line) for methanol CC clusters (left) and water clusters (right) as defined in the text. The dashed lines show the predicted cluster size distribution at the percolation threshold[92].

obtained from the EPSR ensembles (for $x=0.50$ ) are shown on the left of Figure 7.2. At this concentration the methanol in the solution is fully percolating.

The cluster size distributions as obtained from the EPSR ensembles (for $x=0.50$ ) are shown on the right of Figure 7.2 for water clusters. The predicted power law $n_{s} \approx s^{-2.2}$ for random percolation on a 3-d cubic lattice[92] is also shown on Figure 7.2 , illustrated as a dashed line. The number of clusters containing $s$ molecules is plotted as a fraction of total number of clusters, $n(s) / N$ (where $N=\sum_{s} n(s)$ ) against the cluster size $s$. The water cluster distributions show an enhanced probability of the largest water clusters on compression. The system exhibits larger water clusters and these clusters are more frequently present upon compression. There are fewer small, non-percolating clusters as the system is compressed. This is consistent with increased segregation of the two components upon compression.

By examining the distribution of clusters in the solution it has been found that there is enhanced segregation of methanol and water as the system is compressed. There 
are more percolating water clusters and fewer non-percolating water clusters in the solution on compression. Now the local structure of the system will be explored to determine if there are any further indications of enhanced micro-heterogeneity.

\subsection{The Hydrophobic Effect Under Pressure}

The hydrophobic effect is thought to drive the association of non-polar species and in doing so 'releasing' water that would otherwise be in the hydration shell of each non-polar species. In associating, this 'released' hydration water should enable the water network to become more extended or more disorderd. Now, let us consider the structural behaviour of the non-polar species in the methanol-water solution. Examine first the correlations between methanol carbon atoms, where a carbon atom can be thought of as the molecular centre of the alcohol methyl group. Figure 7.3 shows the radial distribution function $g_{C C}$ for $x=0.50$ at $200 \mathrm{~K}$ and 1 bar (black solid line), $x=0.50$ at $200 \mathrm{~K}$ and $2 \mathrm{kbar}$ (black dashed line) and pure methanol (red solid line) for comparison.

From the previous chapter it is known that adding water to methanol at room temperature results in a contraction of the first peak in $g_{C C}$. This has also been observed in other solutions of methanol and water at different mole fraction $(x=0.70[12]$ and $x=0.05[14])$. This contraction of the first peak is believed to be an indication of nonpolar group association in the mixture, where the methyl groups are coming closer together. In the previous chapter, solute association observed for $x=0.54$ at room temperature was seen to reverse on cooling. Figure 7.3 again illustrates that cooling a methanol water systems results in a reverse of this solute association. The first peak position has shifted inward slightly to $\sim 3.87 \AA$ compared to $\sim 3.95 \AA$ for pure methanol. Adding water to methanol at this concentration at low temperature causes a contraction of the non-polar species compared to pure methanol, while it shows re- 
traction of the non-polar species compared to the system at room temperature.

This value for the peak position agrees very well with the results from the previous chapter. In Chapter 7 the system was methanol-water $x=0.54$ mole fraction at 298 $\mathrm{K}$ and $190 \mathrm{~K}$. At the low temperature of $190 \mathrm{~K}$ the first peak position in $g_{C C}$ was $\sim 3.88 \AA$. This is very close to the value obtained for the system described in this chapter, $x=0.50$ at $200 \mathrm{~K}$. Therefore, two completely separate neutron diffraction experiments on two methanol-water solutions at similar state points are showing very good agreement on local structure information. This gives great confidence in the experimental procedure and data analysis technique.

When the pressure is increased to $2 \mathrm{kbar}$ this retraction is reversed and the first peak position in $g_{C C}$ shifts inward to $\sim 3.74 \AA$. Therefore, the effect of increasing the pressure from ambient to $2 \mathrm{kbar}$ is competing with the effect of lowering the temperature. Adding water to methanol enhances the non-polar group association. Cooling the methanol-water system at this concentration causes a retraction of this association. Finally, compression competes with cooling to again enhance non-polar group association. A further insight into the molecular arrangement can be achieved by considering the coordination numbers of the first shell in $g_{C C}$. Integration under the first peak in Figure 7.3 yields a coordination number of 8.19 for $x=0.50$ at $200 \mathrm{~K}$ and ambient pressure and 9.01 for $x=0.50$ at $200 \mathrm{~K}$ and $2 \mathrm{kbar}$. Therefore, increasing the pressure on this cooled methanol-water system has resulted in more methyl group contact in the first coordination shell.

This interesting behaviour is for first neighbour distances in the methanol-methanol correlations. Consider what is happening at greater distances from the first coordination shell. For $x=0.50$ at $200 \mathrm{~K}$ and ambient pressure the second shell peak position in $g_{C C}$ is not changed much compared to $\sim 7.69 \AA$ in pure methanol (Figure 7.3). As the pressure increases to 2 kbar the second peak position in $g_{C C}$ shifts inward significantly to $\sim 6.96 \AA$. This indicates that there is enhanced methanol association in the 


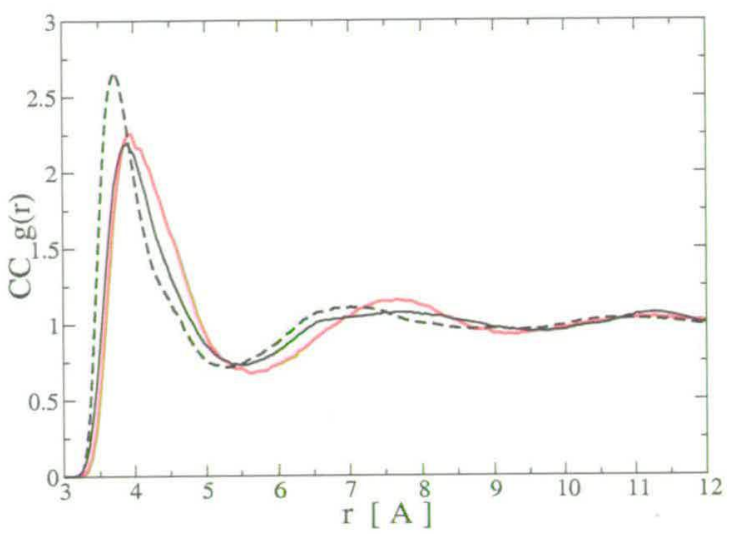

Figure 7.3: Partial radial distribution function for $\mathrm{CC}$ correlations in a methanol-water $\mathrm{x}=0.50$ mole fraction mixture. Graph shows mixture at $200 \mathrm{~K}$ and ambient pressure (black solid line), mixture at $200 \mathrm{~K}$ and $2 \mathrm{kbar}$ (black dashed line) and pure methanol at $298 \mathrm{~K}$ (red line).

mixture to second neighbour level when the system is compressed.

Therefore, compression appears to have two major effects on non-polar correlations. At first neighbour level, compression reverses the effects of lowering the temperature. Cooling a methanol water mixture leads to enhanced association of non-polar groups compared to pure methanol but reduced association compared to the mixture at room temperature, while compression leads to further enhancement of solute association of non-polar groups. The effect of pressure on solute association is influenced more by pressure than by the addition of water. At second neighbour level, compression further enhances the association of non-polar groups. This would suggest that the hydrophobic effect is increasing under pressure. It seems that methanol is soaking up the pressure in this system. In fact, in proteins pressure is thought to decrease the hydrophobic interaction. This will be discussed further at the end of this chapter.

\subsection{Water Under Pressure}

Consider now what happens to the structure of water under pressure. This can be explored by looking at the correlations between water oxygen atoms, where an oxygen 


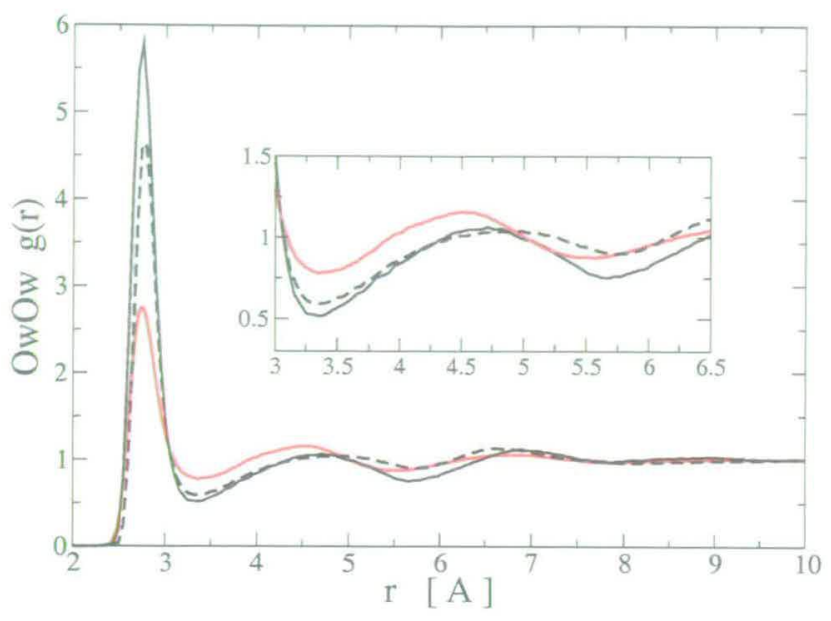

Figure 7.4: Partial radial distribution function for OwOw correlations in a methanol-water $\mathrm{x}=$ 0.50 mole fraction mixture. Graph shows mixture at $200 \mathrm{~K}$ and ambient pressure (black solid line), mixture at $200 \mathrm{~K}$ and $2 \mathrm{kbar}$ (black dashed line) and pure methanol at $298 \mathrm{~K}$ (red line).

atom can be thought of as the molecular centre of the water molecule. Figure 7.4 shows the radial distribution function $g_{O w O w}$ for $x=0.50$ at $200 \mathrm{~K}$ and 1 bar (black solid line), $x=0.50$ at $200 \mathrm{~K}$ and $2 \mathrm{kbar}$ (black dashed line) and pure methanol (red solid line) for comparison.

The position of the first peak in $g_{O w O w}$ remains unchanged. It is the second peak which shows interesting behaviour. In pure water the second peak position of $g_{O w O w}$ is at $\sim$ $4.50 \AA$. In the previous chapter it was found that the addition of methanol to water at $x=0.54$ resulted in a shift of the second peak to larger $r$ values $(\sim 4.88 \AA)$. The methanol in the system is disrupting the water network. On cooling this peak returned to a lower $r$ value of $\sim 4.80 \AA$. For the system of interest in this chapter, $x=0.50$ at $200 \mathrm{~K}$, the second peak position is at $\sim 4.70 \AA$ in very good agreement with the $x=0.54$ system.

The additional constraint of compression yields a very subtle change in the second peak position of $g_{O w O w}$. The peak position shifts back outwards to larger $\mathrm{r} \sim 4.88$ $\AA$. Therefore, compression appears to have the reverse effect to cooling at the second neighbour level in water structure. The position of the second peak in the methanolwater $x=0.50$ is interesting for another reason. This large value of $r$ is greater 
Portial $g(r)$ 's for LDA 80K

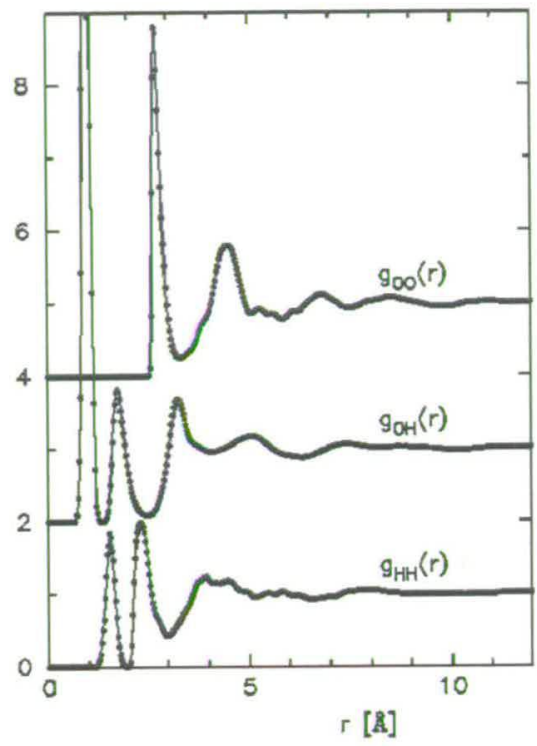

Figure 7.5: Partial radial distribution functions for Low Density Amorphous (LDA) Ice. Top distribution is $\mathrm{OO}$ correlations, middle distribution shows $\mathrm{OH}$ correlations and lower distribution shows $\mathrm{HH}$ correlations, where $O$ represents water oxygen atoms and $H$ represents water hydrogen atoms.

than that of low density water (LDW). Neutron diffraction experiments have provided structural information on the partial radial distribution functions of water in its low density water (LDW) and high density water (HDW) form. Figure 7.5 shows the partial radial distribution functions for Low Density Water (LDW). The top distribution is OO correlations, the middle distribution shows $\mathrm{OH}$ correlations and the lower distribution shows $\mathrm{HH}$ correlations, where $O$ represents water oxygen atoms and $H$ represents water hydrogen atoms. In the case of LDW, the second coordination shell or peak position in $g_{O O}$ is found at $\sim 4.75 \stackrel{\AA}{A}$. This corresponds to a very open water network. In the methanol-water $x=0.50$ system at $200 \mathrm{~K}$ and $2 \mathrm{kbar}$ the second coordination shell or peak position in $g_{O w O w}$ is found at $\sim 4.88 \AA$

Interestingly, there is no significant change in the radial distribution function of interspecies contacts, $g_{C O w}$ with increased pressure (Figure 7.6). The first peak position shifts outwards to larger $\mathrm{r}$ very slightly. This suggests that it is the methanol component 


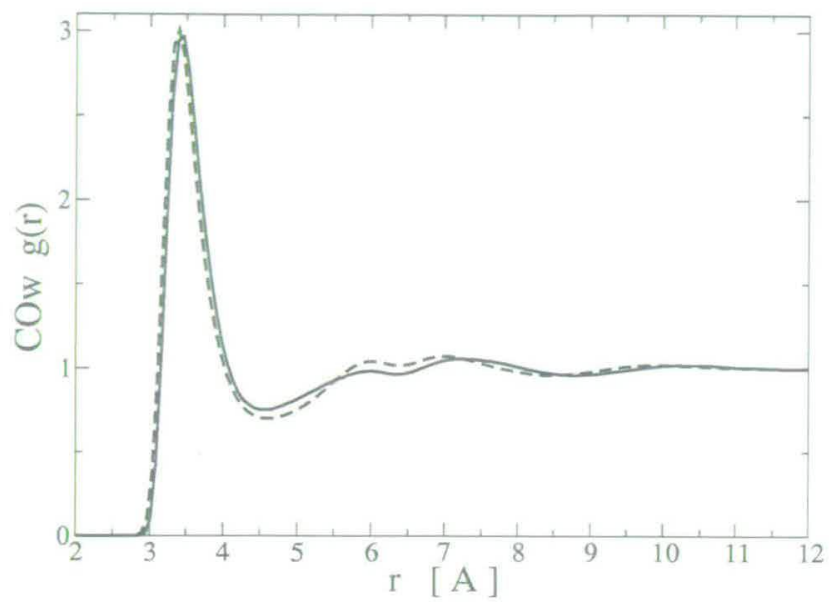

Figure 7.6: Partial radial distribution function for $\mathrm{CO}$ correlations in a methanol-water $\mathrm{x}=0.50$ mole fraction mixture. Graph shows mixture at $200 \mathrm{~K}$ and ambient pressure (black solid line) and mixture at $200 \mathrm{~K}$ and $2 \mathrm{kbar}$ (black dashed line).

of the system which is 'soaking-up' all the pressure.

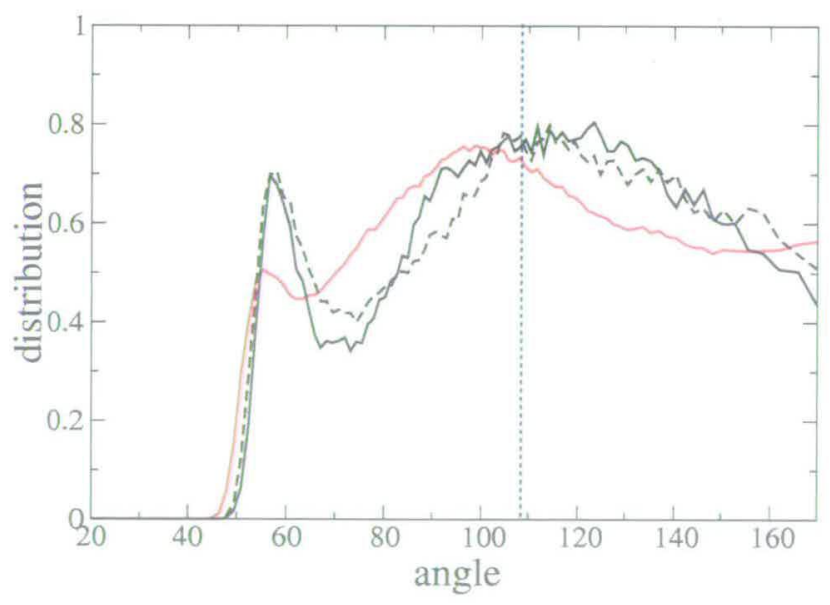

Figure 7.7: The triplet bond angle distribution for $O_{W} O_{W} O_{W}$ for pure water at $298 \mathrm{~K}$ (solid red line), methanol-water $x=0.50$ at $200 \mathrm{~K}$ and ambient pressure (solid black line) and $200 \mathrm{~K}$ and 2 kbar (dashed black line). 


\subsection{Discussion}

Compression of the aqueous alcohol solution leads to the same effect on the mediumrange order of the system, that is to enhance segregation by formation of larger water clusters. However, the local structure of water shows little if any change in the positions of the first peak or coordination shell in $g_{O w O w}(r)$. It is the second coordination shell which suggests a more open, extended water network, one which is comparable to that of the low density form of water. In contrast, the corresponding RDF for methanol carbon atoms in systems at elevated pressure is displaced to lower $r$. It would seem therefore that the topology of the larger clusters formed by enhanced pressure is different to those formed by lowered temperature. The water contained within them does not show a significant trend in the RDF back toward that of bulk water (as was the case for lowered temperature). Therefore, it seems that while there is enhanced segregation under compression, the behaviour of the water is different, perhaps indicating a different topology of the water clusters. The position of the 2 nd peak in $g_{O w O w}(r)$ might suggest that this new topology is such that the water network is much more open and extended, allowing it to penetrate the mixture much better. This will be explored further in the next chapter.

This enhanced segregation at elevated pressure is in contrast to the results of molecular dynamics simulations of Hummer et al [112][113] who have concluded that pressure destabilises the contact configuration of non-polar molecular groups, relative to a solvent-separated configuration. Figure 7.8 shows a snapshot from molecular dynamics simulations of an aqueous solution of 10 hydrophobic solutes (methane) in 508 waters at $1 \mathrm{~atm}$ (top panel) and $8000 \mathrm{~atm}^{1}$ (lower panel)[113]. They found that approximately 7-9 solutes at $1 \mathrm{~atm}$ aggregate to form a hydrocarbon assembly similar to that observed by Wallqvist[114]. At 8000 atm, the solutes sample contact configurations but no aggregate is observed. These authors then assert that pressure denat-

\footnotetext{
${ }^{1}$ Where 1 atm (atmosphere) is $\sim 1.01$ bar
} 
uration of proteins proceeds by a similar mechanism, that is solvent penetration into a hydrophobic core. With increasing pressure, the transfer of water into the protein interior becomes key to the pressure denaturation process, leading to the dissociation of close hydrophobic contacts and subsequent swelling on the hydrophobic protein interior through insertions of water molecules. The protein core continues to swell until the protein unfolds. In contrast to this the results from diffraction measurements and from recent molecular dynamics simulations [115] indicate the hydrophobic groups get pushed closer together with pressure. This difference may be due to the consequence of having an amphiphile in solution rather than a simple hydrophobe. It also seems strange that water want to go into the hydrophobic core.

Interestingly, in both this work and the methane water simulations[112] the effect of pressure is to enhance the water structure. It might have been expected that pressure would break up the water structure. Instead, in these mixtures[112] and the methanolwater system the effect of pressure is to enhance the water structure. Water is able to penetrate into the clusters better at high pressure. This would indicate a change in the topology of the water clusters. It appears that pressure is somehow stabilising the micro-segregation observed in methanol-water systems, allowing it to extend further. 

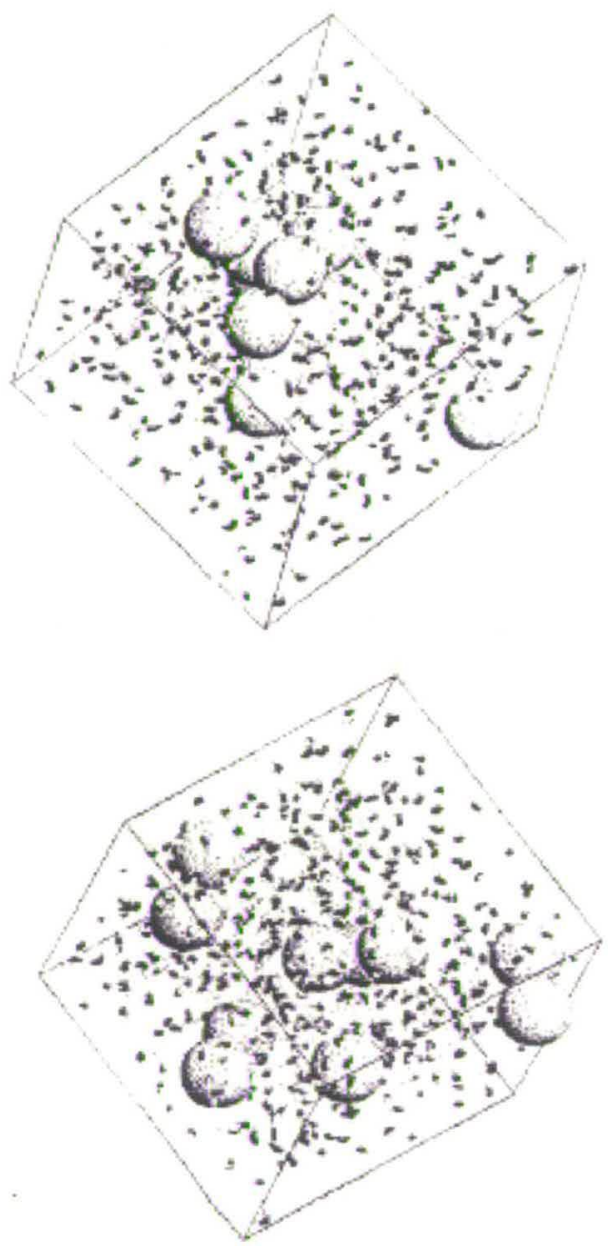

Figure 7.8: Snapshot from molecular dynamics simulations of an aqueous solution of 10 hydrophobic solutes (methane) in 508 waters at 1 atm (top panel) and 8000 atm (lower panel)[113]. 


\section{Chapter 8}

\section{A Simple Model to Explain}

\section{Observed Entropy}

\subsection{Motivation}

In spite of the considerable work devoted to the study of the hydrophobic effect, there is still no general agreement about the validity of the Frank and Evans 'iceberg' model[10]. Alternative models have been proposed[23],[116],[117] but a complete understanding of the hydrophobic phenomenom has not yet been achieved. In particular, no structural enhancement is observed in the experimentally determined radial distribution functions of water in the presence of non-polar groups[26], [12], [89], [27], [14] and [75]. Early simulations seemed to support the idea of water structure enhancement, however, newer models and simulations were not able to find any evidence of the structure of water becoming more ordered. In particular, the effect of the solute on the structure of the solvent[118], [119], [120] and the aggregation of solutes in water[111],[121] have received a great deal of attention.

This chapter proposes that the excess entropy in such solutions can be understood in terms of a simple excluded volume argument, where the molecular scale segregation in 


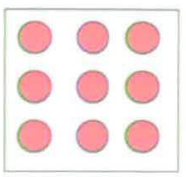

Liquid A

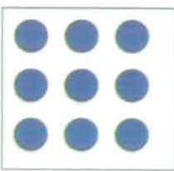

Liquid B

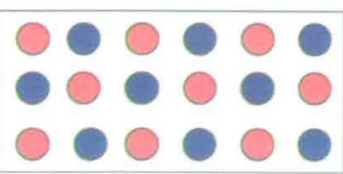

Liquid A + B

Figure 8.1: Schematic of ideal mixing of two liquids $A$ and $B$ to form a homogeneous mixture

these systems is sufficient to explain the observed excess entropies. This chapter begins with a reminder of the meaning negative excess entropy. It then proceeds by estimating the entropy assuming complete demixing of the two components at the microscopic level. It then develops this simple model to approach micro-segregation in methanolwater systems.

\subsection{Calculating the Excess Entropy}

For an ideal solution, when two liquids $A$ and $B$ are mixed the Gibbs free energy of mixing is given by

$$
\triangle G_{\operatorname{mix}}=R T\left(x_{A} \ln x_{A}+x_{B} \ln x_{B}\right)
$$

where $x_{A}$ is the mole fraction of liquid $A$ and $x_{B}$ the mole fraction of liquid $B$.

Ideal systems are defined as those for which intermolecular interactions (enthalpic contributions) are negligible. In ideal systems there are interactions, but the average A-B interactions in the mixture of liquid A and B are the same as the average A-A and B-B interactions in the pure liquids. Therefore the driving force for mixing is the increasing entropy of the system as the molecules mingle and the enthalpy of mixing is zero. In ideal systems homogeneous mixing is always favoured where the properties of the solution are the same for all regions within the occupied volume (Figure 8.1).

The ideal entropy of mixing of the two liquids $\mathrm{A}$ and $\mathrm{B}$ is then: 


$$
\triangle S_{m i x}=-R\left(x_{A} \ln x_{A}+x_{B} \ln x_{B}\right)
$$

or

$$
\triangle S_{m i x}=-k N \sum_{j} x_{j} \ln x_{j}
$$

Since for any mixture the mole fraction of component $j, x_{j}=\frac{N_{j}}{N}$, will be less than unity the entropy given by equation 8.3 must always be positive compared to either of the pure components on their own.

If the number of molecules of component $j$ in a mixture is $N_{j}$, the volume of the mixture is $V$, and each component is randomly distributed throughout the volume, the local density per unit volume of component $A$ in the mixture is equal to the mean density of that component.

$$
\rho_{j}=\bar{\rho}_{j}=\frac{N_{j}}{V}
$$

Then, in terms of the density, the entropy of this ideal mixture is given by

$$
\triangle S_{m i x}=-k \sum_{j} \int_{V} \bar{\rho}_{j} \ln \frac{\bar{\rho}_{j}}{\bar{\rho}}
$$

The mean density of all the molecules in the mixture $\bar{\rho}$ is given by

$$
\bar{\rho}=\frac{N}{V}
$$

and the number of molecules in the mixture is $N=\sum_{j} N_{j}$.

It has been known for some time that the observed excess entropies of mixing molecules with hydrophobic headgroups with water are significantly less than their ideal values[10]. 


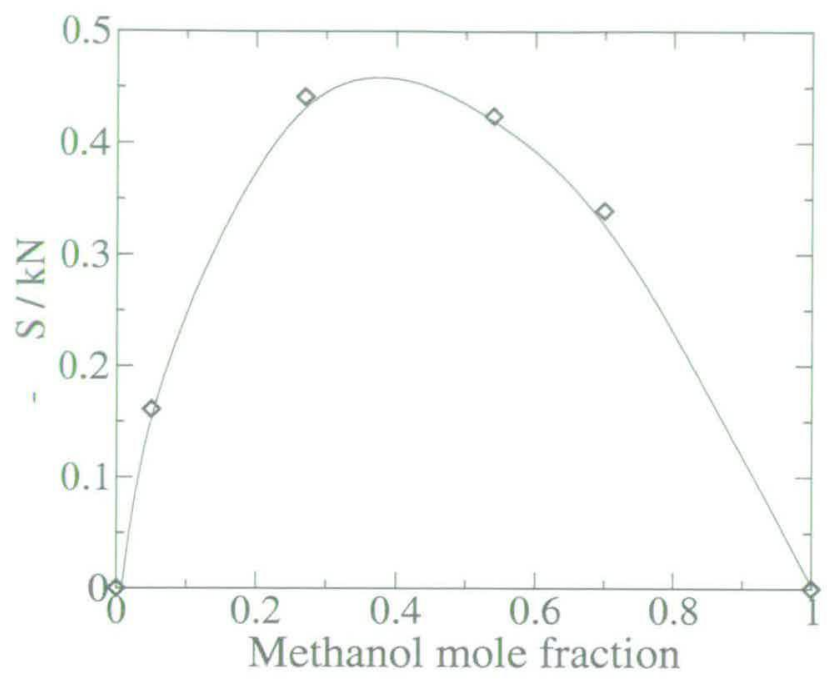

Figure 8.2: Experimentally measured negative excess entropies, $-\frac{\Delta S}{k N}$ for methanol-water solutions at a range of methanol mole fractions[71]

Figure 8.2 shows the experimentally observed excess entropy for methanol-water solutions as a function of methanol mole fraction[71].

Now consider a system which is made up of two components which are completely demixed at the microscopic level. In other words, there is a sharp boundary between water regions and methanol regions (Figure 8.3). In this case, the local density of $j$ molecules $\rho_{j}$ is either $\sim \frac{1}{v_{j}}$, where $v_{j}$ is the molar volume of component $j$ in the pure form, or zero. The molar volume of a liquid is the volume taken up by one mole. According to equation 8.5 and 8.3 regions of zero density[122] will not contribute to the entropy. Writing $\bar{\rho}=\frac{1}{\bar{v}}$, where $\bar{v}=\sum_{j} x_{j} v_{j}$ is the mean molar volume of the mixture, then the excess entropy relative to ideal mixing becomes

$$
\Delta S=-k\left\{\sum_{j}\left[\int d V \rho_{j} \ln \frac{\rho_{j}}{\bar{\rho}}\right]-N \sum_{j} x_{j} \ln x_{j}\right\}
$$

which can be written as

$$
\Delta S=-k N \sum_{j} x_{j} \ln \left(\frac{\bar{v}}{x_{j} v_{j}}\right)
$$




\begin{tabular}{|c|c|c|c|c|c|c|c|}
\hline$x_{m}$ & $\begin{array}{c}\bar{v} \\
{\left[\AA^{3}\right]}\end{array}$ & $\frac{\bar{v}}{x_{m} v_{m}}$ & $\frac{\bar{v}}{x_{w} v_{w}}$ & $\begin{array}{c}-\frac{T \Delta S_{\text {excess }}}{n} \\
{[\mathrm{~kJ} / \mathrm{mole}]}\end{array}$ & $\begin{array}{c}\text { Experiment } \\
{[\mathrm{kJ} / \mathrm{mole}]}\end{array}$ & $\begin{array}{c}-\frac{\Delta S}{k N} \\
\text { units }\end{array}$ & $\begin{array}{c}\text { Experiment } \\
\text { units }\end{array}$ \\
\hline 0.00 & 30.0 & - & 1.000 & 0.000 & 0.000 & 0.000 & 0.000 \\
0.05 & 31.9 & 9.382 & 1.119 & 0.542 & 0.399 & 0.22 & 0.16 \\
0.27 & 40.3 & 2.193 & 1.838 & 1.626 & 1.092 & 0.66 & 0.44 \\
0.54 & 50.5 & 1.376 & 3.661 & 1.906 & 1.050 & 0.77 & 0.42 \\
0.70 & 56.6 & 1.189 & 6.289 & 1.667 & 0.840 & 0.67 & 0.34 \\
1.00 & 68.0 & 1.000 & - & 0.000 & 0.000 & 0.000 & 0.000 \\
\hline
\end{tabular}

Table 8.1: Calculation of the negative excess entropy of methanol-water solutions on the basis of a simple model that assumes all the molecules of each component occur in completely segregated clusters of the same density as the pure liquid counterparts. The experimentally observed are also shown for reference[71]

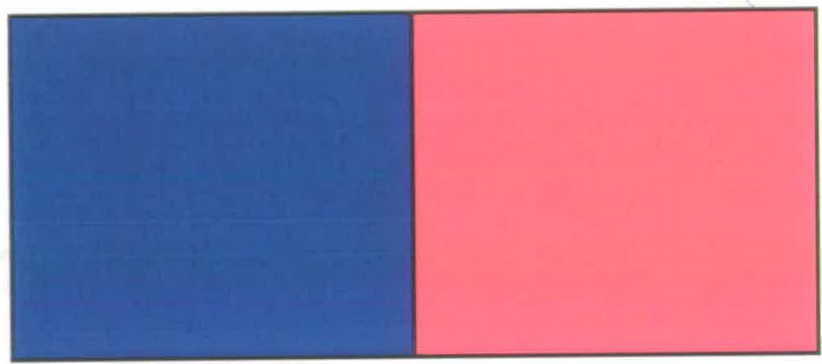

Figure 8.3: Schematic illustrating the case of complete demixing of methanol (shown in red) and water (shown in blue)

For the pure liquids the molar volume of water $v_{w} \sim 30 \AA^{3}$ at $298 \mathrm{~K}$, while for methanol the molar volume is $v_{m} \sim 68 \AA^{3}$ at $298 \mathrm{~K}$. In the mixture these values are not appreciably different[123], [124], [125] from the pure liquid values.

These values for the molar volumes of the two liquids can be used to calculate the excess entropy, $\triangle S$, given by equation 8.8 at a range of different compositions of mole fraction. These values for $\triangle S$ are shown in Table 8.1 and the results are compared with the measured excess entropies for methanol-water. It can be seen that the qualitative trend is reproduced, but quantitatively the results are too large by a factor of $\sim 2$. Therefore, the negative excess entropies produced from using equation 8.8 are very 


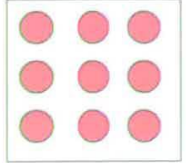

Liquid A

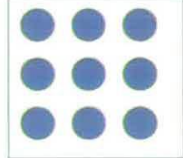

Liquid B

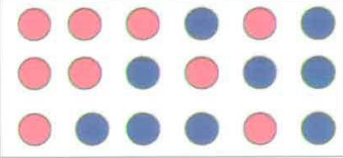

Liquid A + B

Figure 8.4: Schematic of non-ideal mixing of two liquids $A$ and $B$ to form an inhomogeneous mixture

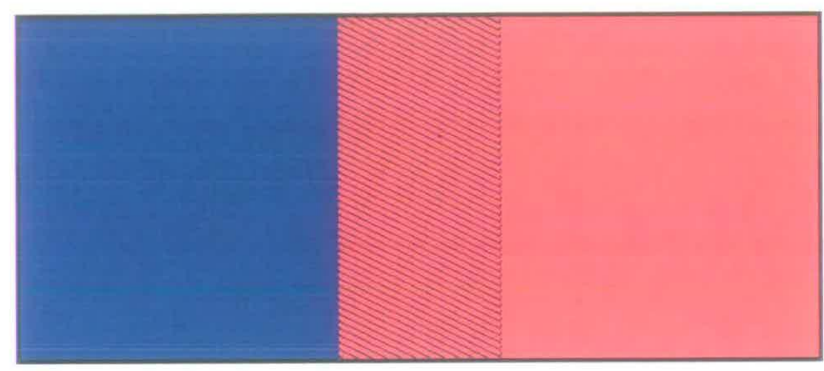

Figure 8.5: Schematic illustrating a methanol (shown in red) and water (shown in blue) mixture which includes an interfacial region of methanol and water (blue and red hatched region)

large and can be thought of as upper limits.

Real solutions are composed of particles for which A-A, B-B and A-B interactions are all different. Therefore there will be an enthalpy change $\left(\triangle H_{m i x}\right)$ when the liquids are mixed. There will also be an additional contribution to the entropy arising from the way in which the molecules of one type might cluster together instead of mingling freely with the others (Figure 8.4).

Equation 8.8 makes no reference to the detailed nature of the clustering, nor to any structural changes that may take place in methanol and water. The only assumption is that there is a sharp boundary between water and alcohol clusters i.e. no mixing at the atomic level. In practise the interface between alcohol and water regions will be more diffuse, comprising regions in which the two species intermingle via hydrogen bonding, creating the equivalent of random mixing in this region (Figure 8.5). These interface regions will therefore contribute little or nothing to the excess entropy.

If, of the $N_{j}$ molecules of type $j$ a fraction $f_{j}$ are found, by some criterion to be specified, to be interfacial molecules then the excess entropy function becomes 


$$
\triangle S=-k N\left\{\sum_{j} x_{j}\left(1-f_{j}\right) \ln \left(\frac{\bar{v}}{v_{j}}\right)+\sum_{j} x_{j} f_{j} \ln x_{j}-\sum_{j} x_{j} \ln x_{j}\right\}
$$

and therefore

$$
\triangle S=-k N \sum_{j} x_{j}\left(1-f_{j}\right) \ln \left(\frac{\bar{v}}{x_{j} v_{j}}\right)
$$

The overall entropy in the system is therefore raised towards the random mixing level (i.e. away from complete demixing), leading to less negative excess entropy.

The fraction of interfacial molecules $f_{j}$ can be obtained experimentally through the combined procedures of neutron diffraction (with isotope substitution) and Empirical Potential Structural Refinement (EPSR). The EPSR procedure has been explained in detail in Chapter 4. This combination of techniques provides access to all the structural correlations, from the experimentally constrained EPSR ensembles. Molecules are then assigned to be either within a cluster or in the interface between clusters, using simple distance criteria. These molecules in the interface are then the interfacial molecules which contribute to the fraction of molecules involved in random mixing.

Methanol and water clusters are defined in the same way as was described in the cluster analysis distribution section. To recap, methanol molecules are identified by the requirement that any two carbon atoms separated by $5.6 \AA$ or less are assumed to be in the same cluster. This is the position of the first minimum in the carbon-carbon radial distribution function, $g_{C C}$. Two water molecules are assumed to be in the same cluster if their oxygen atoms are $3.5 \AA$ or less apart.

To estimate the fraction of molecules in the interfacial region, three different criteria are adopted. These criteria are:

(a) Based on carbon, $C$, to water oxygen, $O w$ distance in radial distribution function $r_{C O w}$ 
(b) Based on either the methanol oxygen, $\mathrm{O}$, to water hydrogen, $\mathrm{Hw}$, in radial distribution function $r_{\mathrm{OH} w}$ or the methanol hydroxyl hydrogen, $\mathrm{H}$ to water oxygen, $\mathrm{Ow}$, in radial distribution function $r_{H O w}$

(c) Based on either the $g_{C O w}$ distance or $r_{O H w}$ distance or $r_{H O w}$ distance in radial distribution function

Criterion $(a)$ therefore represents a general specification for the distance of approach of a water molecule to a methanol molecule for the two molecules to be assigned to the interfacial region. Criterion $(b)$ refers specifically to water hydrogen bonding to a methanol molecule, either through the water hydrogen atom or the methanol hydrogen atom. Criterion $(c)$ as a combination of these two criteria.

Specific distances that were tried for criterion $(a)$ were $3.20,3.25$ and $3.30 \AA$ for $r_{C O w}$, for criterion $(b) 1.75,1.80$ and $1.85 \AA$ for both $r_{O H w}$ and $r_{H O w}$, and for criterion (c) $1.70,1.75$ and $1.80 \AA$ for both $r_{O H w}$ and $r_{H O w}$, with $r_{C O w}$ set to $3.1 \AA$.

All three sets of distances are close to or slightly below the position of the first peak in the corresponding radial distribution functions, indicating that the overall thickness of the interfacial region is about the mean separation of a pair of molecules.

\subsection{Excess Entropy of Methanol-Water Mixtures}

The fraction of molecules in the interfacial region was found using the specified criteria and from this the excess entropy was calculated using equation 8.10. Table 8.2 shows the surface fractions and derived excess entropies (tabulated as $-\frac{\Delta S}{k N}$ ) for one of criterion $(a)$ for a number of different compositions of methanol and water; mole fraction $x=0.05, x=0.27, x=0.54$ and $x=0.70$. The table also shows the experimentally observed excess entropy for these concentrations[71]. These results are shown in Figure. It is clear that this simple method is readily able to explain the 


\begin{tabular}{|c|c|c|c|c|c|c|c|c|c|}
\hline $\begin{array}{c}r_{C O w} \\
{[\AA]}\end{array}$ & $\begin{array}{c}\text { Methanol } \\
\text { mole fraction }\end{array}$ & $\begin{array}{c}0.05 \\
\mathrm{Cal}\end{array}$ & $\begin{array}{c}0.05 \\
\text { Expt }\end{array}$ & $\begin{array}{c}0.27 \\
\mathrm{Cal}\end{array}$ & $\begin{array}{c}0.27 \\
\text { Expt }\end{array}$ & $\begin{array}{c}0.54 \\
\mathrm{Cal}\end{array}$ & $\begin{array}{c}0.54 \\
\text { Expt }\end{array}$ & $\begin{array}{c}0.70 \\
\mathrm{Cal}\end{array}$ & $\begin{array}{c}0.70 \\
\text { Expt }\end{array}$ \\
\hline 3.30 & $f_{m}$ & 0.798 & & 0.593 & & 0.405 & & 0.285 & \\
& $f_{w}$ & 0.077 & & 0.268 & & 0.466 & & 0.553 & \\
\hline & $-\frac{\Delta S}{k N}$ & 0.122 & 0.161 & 0.485 & 0.441 & 0.421 & 0.424 & 0.333 & 0.339 \\
\hline
\end{tabular}

Table 8.2: Estimated surface fractions and modified excess entropy of methanol-water solutions, after correcting for the fraction of methanol $\left(f_{m}\right)$ and water $\left(f_{w}\right)$ molecules that occur in the interfacial regions of the respective clusters.

observed excess entropies both qualitatively as well as quantitatively.

The calculation relies on the fact that the system is clustered, and the result will depend on the size and shape of the clusters. A few large clusters of globular shape would give rise to relatively few interfacial molecules. On the other hand, sheet-like clusters or a large number of small clusters would have a much higher percentage of surface molecules, leading to less negative excess entropy. In this case the system would be more disordered and therefore the entropy would be greater than in the example of the demixed situation. Therefore the nature of the clustering has a direct impact on the observed excess entropy. At the same time the values of the separation distances (only one example is shown in Table 8.2) needed to achieve the correct entropy values probably don't have much intrinsic significance, except that they do correspond to distances near the main peak of the corresponding radial distribution function.

Criterion $(b)$ and $(c)$ which are based on the hydrogen bonding between methanol and water, give the best overall agreement in the concentration dependence of the excess entropy. The primary driver behind the observed clustering is believed to be the readiness of water molecules to form hydrogen bonds with the methanol hydroxyl group. 


\subsection{Influence of Cooling on Excess Entropy}

Now consider the influence of cooling methanol-water systems on the observed excess entropy. Tables 8.3 and 8.4 show the fraction of interfacial molecules and derived excess entropies (presented as $-\frac{\Delta S}{k N}$ for clarity) for criterion $(a),(b)$ and $(c)$. The change in the excess entropy with cooling is shown in Figure 8.6. Experimentally measured values for the excess entropy are shown with black line and diamonds, values from the model with complete demixing are shown as red line with squares, values from model using $C O_{W}=3.30 \AA$ definition of clusters are shown as green line with circles and the low temperature measurements are shown as orange triangles. Two different systems were studies; methanol-water $x=0.27$ mole fraction at $293 \mathrm{~K}$ and $238 \mathrm{~K}$ and methanol-water $x=0.54$ mole fraction at $298 \mathrm{~K}, 260 \mathrm{~K}$ and $190 \mathrm{~K}$.

Consider first the $x=0.27$ mole fraction solution. As the mixture is cooled the fraction of interfacial molecules for both methanol and water decreases. Therefore, there are more methanol molecules which are members of methanol clusters and more water molecules which are members of water clusters. Next, consider the derived negative excess entropy. For the methanol water $x=0.27$ system at $293 \mathrm{~K}$ the $-\frac{\Delta S}{k N} \sim 0.52$. This is a less negative value than that obtained from a completed demixed solution (Table 8.1) as expected, since some of the methanol and water will be in interfacial regions. Consider what happens when the system is cooled. The excess entropy values for the $x=0.27$ methanol-water mixture increases as the mixture is cooled, i.e. $-\frac{\Delta S}{k N}$ becomes larger, $\sim 0.56$ at $238 \mathrm{~K}$. Therefore, as the mixture is cooled the reduction of molecules in the interface leads to greater micro-segregation of the 2 components and therefore $-\frac{\Delta S}{k N}$ starts to approach the 'demixed' value calculated previously.

This is consistent with the results from previous work. From the cluster distribution analysis it was clear that cooling the methanol-water $x=0.27$ mixture increased the segregation of the mixture. The frequency of the probability of percolating clusters increased and cluster size increased with cooling. This would imply that as the clus- 


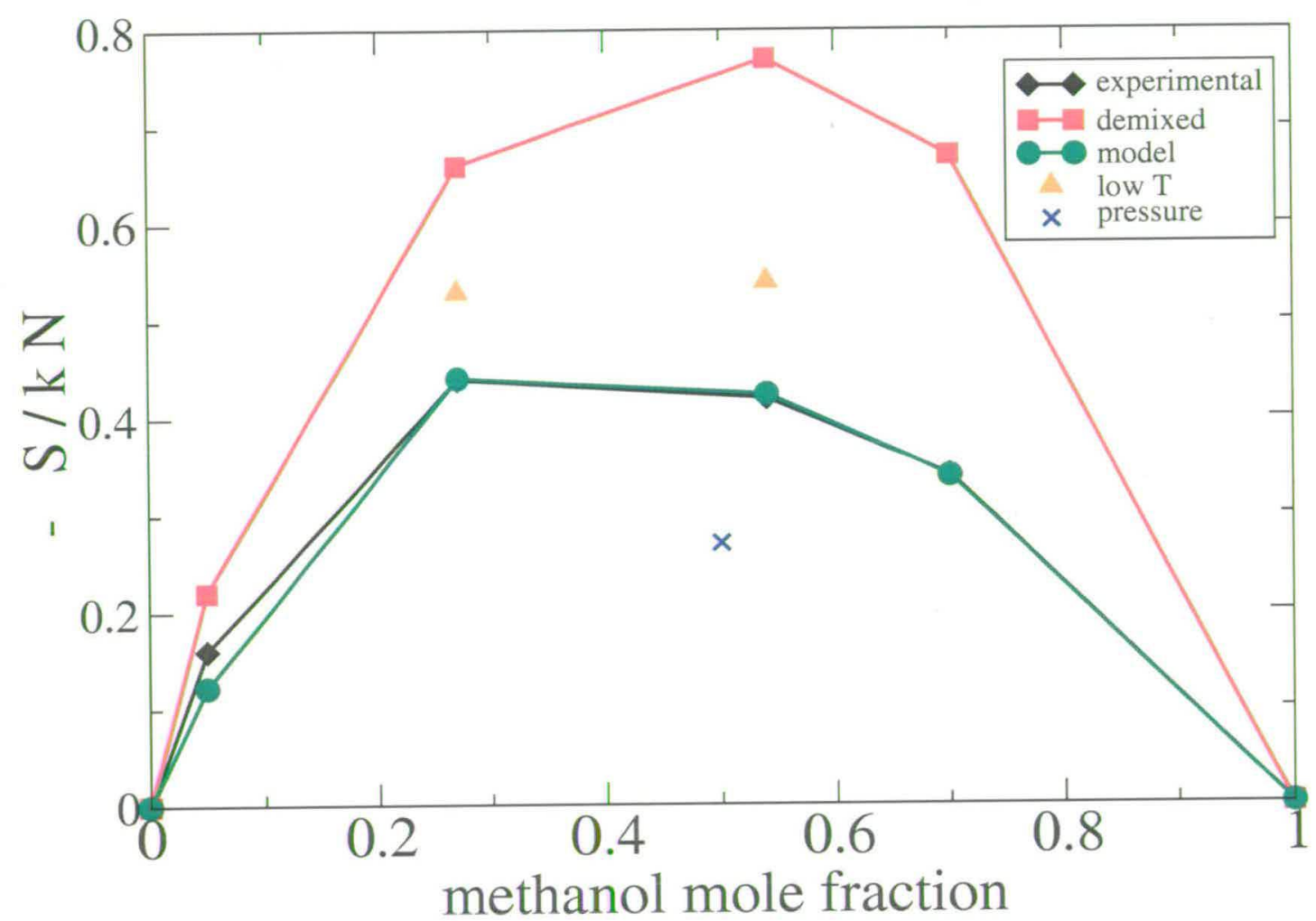

Figure 8.6: Excess entropy for a range of different methanol water mixtures from experimentally measured values (black line with diamonds), from model with complete demixing (red line with squares), from model using $C O_{W}=3.30 \AA$ definition of clusters (green line with circles), for low temperature measurements (orange traingles) and low temperature and high pressure (blue cross) 
ters were more enhanced with cooling, then the liquid structure was becoming more ordered As a result of this clustering or micro-segregation, there are less interfacial molecules in the solution. This reduction in the random mixing of the interfacial region results in a reduction in the entropy, seen in the larger negative values for $-\frac{\Delta S}{k N}$. For the methanol-water $x=0.54$ mole fraction system similar trends are observed (Table 8.4). The fraction of interfacial molecules, $f$ decreases as the system is cooled. The negative excess entropy, $-\frac{\Delta S}{k N}$, becomes more negative with cooling changing from $\sim 0.41$ to $\sim 0.53$. The system is again moving towards the demixed state value of excess entropy. On cooling there is more order in the system since there is less disorder or random mixing in interfacial region.

\subsection{Influence of Compression on Excess Entropy}

Finally, consider the influence of pressure on the excess entropy of a methanol-water system. Table 8.5 shows the fraction of interfacial molecules and derived excess entropies (presented as $-\frac{\Delta S}{k N}$ for clarity) for criterion $(a),(b)$ and $(c)$. The change in the excess entropy with compression is shown in Figure 8.6. Experimentally measured values for the excess entropy are shown with black line and diamonds, values from the model with complete demixing are shown as red line with squares, values from model using $C O_{W}=3.30 \AA$ definition of clusters are shown as green line with circles and the low temperature and high pressure measurement is shown as a blue cross. The system studied was methanol-water $x=0.50$ at $200 \mathrm{~K}$ and ambient pressure (1 bar) and methanol-water $x=0.50$ at $200 \mathrm{~K}$ and 2 kbar

With compression the following trends are observed (Table 8.5). The fraction of interfacial molecules $f$ increases when the cooled system is compressed. There are more water molecules and more methanol molecules in the interface. Is this change in $f$ an indication that the structure in the methanol-water system is changing with pres- 
sure? It is known from methanol and water cluster distributions [115] that compressing a cooled methanol-water system leads to enhanced segregation. The frequency of percolating clusters is observed to increase. However, the values of $f$ indicate that the fraction of molecules in the interface of methanol and water is increasing. This may be an indication that the topology of the micro-segregated system changes under compression. There are more surface molecules, which would indicate that the clusters are becoming even more string like or sheet like (would also increase the area of interfacial molecules) hence more surface available for interface.

The negative excess entropy $-\frac{\Delta S}{k N}$ decreases when the cooled system is compressed. The entropy is rising in the system under cooling and compression. This indicates that there is more disorder in the system due to the change in the interfacial region.

\subsection{Summary and Discussion}

Experimental and computational studies have shown that water in the presence of alcohols and other molecules tends to form clusters, rather than being randomly mixed. In this chapter a simple excluded volume argument has been proposed to understand the observed excess entropies in these solutions. The molecular scale segregation in these systems is sufficient to explain the observed excess entropy and there is no need to invoke the 'standard' Frank and Evans iceberg model discussed in Chapter 2.

Using a simple excluded volume argument the excess entropy at extreme conditions can be probed. As a methanol-water mixture is cooled the number of interfacial molecules decreases. This suggests that there are more molecules in clusters and hence more order in the system. The excess entropy of the system falls (becomes more negative) as the solution is becoming more clustered. When a cooled methanol-water mixture is compressed the number of interfacial molecules increases and the excess entropy rises (becomes less negative). It appears that pressure is somehow stabilising 
the micro-segregation observed in methanol-water systems, making it extend further. At the same time it is changing the topology from something closer to $3 \mathrm{D}$ clusters at ambient pressure to more like sheet or chain-like at high pressure. Thus there is a sense in which water has penetrated the clusters, by increasing the fraction of interfacial molecules. It should be noted that the definition of the clusters used in this analysis takes no account of the proximity of neighbouring molecules of the opposite kind. Therefore, the dimensionality of the clusters is not specifically identified.

At the high pressure investigated for this system (2 kbar), no break-up for non-polar clusters were observed, as was in the case of Hummer et al[112] discussed in the previous chapter. It is possible that the break-up of clusters may happen at a still higher pressure in the methanol-water system. This may be a neutron diffraction experiment which is achievable in the near future. This difference in pressure may provide part of the confirmation or extension of Hummer's ideas. Experimentally, this would be very difficult to see in methane itself, since it is difficult to get much methane into solution at ambient temperature and pressure. 


\begin{tabular}{|c|c|c|c|c|c|}
\hline $\begin{array}{c}r_{C O w} \\
{[\AA]}\end{array}$ & $\begin{array}{c}r_{H O w} \\
{[\AA]}\end{array}$ & $\begin{array}{c}r_{O H w} \\
{[\AA]}\end{array}$ & $\begin{array}{c}\text { Methanol } \\
\text { mole fraction }\end{array}$ & $\begin{array}{l}0.27 \\
293 K\end{array}$ & $\begin{array}{l}0.27 \\
238 \mathrm{~K}\end{array}$ \\
\hline \multirow[t]{3}{*}{3.20} & & & $f_{m}$ & 0.30 & 0.19 \\
\hline & & & $f_{w}$ & 0.12 & 0.07 \\
\hline & & & $-\frac{\Delta S}{k N}$ & 0.54 & 0.59 \\
\hline \multirow[t]{3}{*}{3.25} & & & $f_{m}$ & 0.40 & 0.25 \\
\hline & & & $f_{w}$ & 0.16 & 0.09 \\
\hline & & & $-\frac{\Delta S}{k N}$ & 0.50 & 0.56 \\
\hline \multirow[t]{12}{*}{3.30} & & & $f_{m}$ & 0.46 & 0.35 \\
\hline & & & $f_{w}$ & 0.21 & 0.13 \\
\hline & & & $-\frac{\Delta S}{k N}$ & 0.47 & 0.53 \\
\hline & 1.75 & 1.75 & $f_{m}$ & 0.27 & 0.26 \\
\hline & & & $f_{w}$ & 0.11 & 0.10 \\
\hline & & & $-\frac{\Delta S}{k N}$ & 0.55 & 0.56 \\
\hline & 1.80 & 1.80 & $f_{m}$ & 0.32 & 0.32 \\
\hline & & & $f_{w}$ & 0.13 & 0.12 \\
\hline & & & $-\frac{\Delta S}{k N}$ & 0.53 & 0.54 \\
\hline & 1.85 & 1.85 & $f_{m}$ & 0.36 & 0.37 \\
\hline & & & $f_{w}$ & 0.14 & 0.14 \\
\hline & & & $-\frac{\Delta S}{k N}$ & 0.52 & 0.53 \\
\hline \multirow[t]{3}{*}{3.10} & 1.70 & 1.70 & $f_{m}$ & 0.28 & 0.28 \\
\hline & & & $f_{w}$ & 0.12 & 0.11 \\
\hline & & & $-\frac{\Delta S}{k N}$ & 0.54 & 0.55 \\
\hline \multirow[t]{3}{*}{3.10} & 1.75 & 1.75 & $f_{m}$ & 0.35 & 0.32 \\
\hline & & & $f_{w}$ & 0.15 & 0.13 \\
\hline & & & $-\frac{\Delta S}{k N}$ & 0.52 & 0.53 \\
\hline \multirow[t]{3}{*}{3.10} & 1.80 & 1.80 & $f_{m}$ & 0.41 & 0.37 \\
\hline & & & $f_{w}$ & 0.17 & 0.15 \\
\hline & & & $-\frac{\Delta S}{k N}$ & 0.49 & 0.51 \\
\hline
\end{tabular}

Table 8.3: Estimated surface fractions and modified excess entropy of methanol-water solutions, after correcting for the fraction of methanol $\left(f_{m}\right)$ and water $\left(f_{w}\right)$ molecules that occur in the interfacial regions of the respective clusters. 


\begin{tabular}{|c|c|c|c|c|c|c|}
\hline $\begin{array}{c}r_{C O w} \\
{[\AA]}\end{array}$ & $\begin{array}{c}r_{H O w} \\
{[\stackrel{\circ}{A}]}\end{array}$ & $\begin{array}{c}r_{O H w} \\
{[\AA]}\end{array}$ & $\begin{array}{c}\text { Methanol } \\
\text { mole fraction }\end{array}$ & $\begin{array}{r}0.54 \\
298 K\end{array}$ & $\begin{array}{c}0.54 \\
260 K \\
\end{array}$ & $\begin{array}{c}0.54 \\
190 K\end{array}$ \\
\hline \multirow[t]{3}{*}{3.20} & & & $f_{m}$ & 0.22 & 0.19 & 0.19 \\
\hline & & & $f_{w}$ & 0.26 & 0.22 & 0.21 \\
\hline & & & $-\frac{\Delta S}{k N}$ & 0.58 & 0.60 & 0.61 \\
\hline \multirow[t]{3}{*}{3.25} & & & $f_{m}$ & 0.30 & 0.31 & 0.29 \\
\hline & & & $f_{w}$ & 0.37 & 0.34 & 0.31 \\
\hline & & & $-\frac{\Delta S}{k N}$ & 0.50 & 0.51 & 0.61 \\
\hline \multirow[t]{12}{*}{3.30} & & & $f_{m}$ & 0.4 & 0.43 & 0.39 \\
\hline & & & $f_{w}$ & 0.48 & 0.45 & 0.40 \\
\hline & & & $-\frac{\Delta S}{k N}$ & 0.41 & 0.43 & 0.53 \\
\hline & 1.75 & 1.75 & $f_{m}$ & 0.42 & 0.32 & 0.36 \\
\hline & & & $f_{w}$ & 0.48 & 0.37 & 0.4 \\
\hline & & & $-\frac{\Delta S}{k N}$ & 0.41 & 0.49 & 0.46 \\
\hline & 1.80 & 1.80 & $f_{m}$ & 0.47 & 0.39 & 0.45 \\
\hline & & & $f_{w}$ & 0.53 & 0.42 & 0.59 \\
\hline & & & $-\frac{\triangle S}{k N}$ & 0.37 & 0.45 & 0.47 \\
\hline & 1.85 & 1.85 & $f_{m}$ & 0.55 & 0.48 & 0.54 \\
\hline & & & $f_{w}$ & 0.61 & 0.53 & 0.58 \\
\hline & & & $-\frac{\Delta S}{k N}$ & 0.31 & 0.37 & 0.40 \\
\hline \multirow[t]{3}{*}{3.10} & 1.70 & 1.70 & $f_{m}$ & 0.4 & 0.32 & 0.27 \\
\hline & & & $f_{w}$ & 0.46 & 0.36 & 0.32 \\
\hline & & & $-\frac{\Delta S}{k N}$ & 0.43 & 0.50 & 0.53 \\
\hline \multirow[t]{3}{*}{3.10} & 1.75 & 1.75 & $f_{m}$ & 0.46 & 0.38 & 0.39 \\
\hline & & & $f_{w}$ & 0.54 & 0.43 & 0.42 \\
\hline & & & $-\frac{\Delta S}{k N}$ & 0.37 & 0.45 & 0.53 \\
\hline \multirow[t]{3}{*}{3.10} & 1.80 & 1.80 & $f_{m}$ & 0.51 & 0.44 & 0.48 \\
\hline & & & $f_{w}$ & 0.57 & 0.48 & 0.50 \\
\hline & & & $-\frac{\Delta S}{k N}$ & 0.34 & 0.41 & 0.45 \\
\hline
\end{tabular}

Table 8.4: Estimated surface fractions and modified excess entropy of methanol-water solutions, after correcting for the fraction of methanol $\left(f_{m}\right)$ and water $\left(f_{w}\right)$ molecules that occur in the interfacial regions of the respective clusters. 


\begin{tabular}{|c|c|c|c|c|c|}
\hline $\begin{array}{c}r_{C O w} \\
{[\AA]}\end{array}$ & $\begin{array}{c}r_{H O w} \\
{[\AA]}\end{array}$ & $\begin{array}{c}r_{\mathrm{OH} w} \\
{[\AA]}\end{array}$ & $\begin{array}{l}\text { Methanol } \\
\text { mole fraction }\end{array}$ & $\begin{array}{c}0.50 \\
200 \mathrm{~K}\end{array}$ & $\begin{array}{c}0.50 \\
200 \mathrm{~K}, 2 \mathrm{kbar}\end{array}$ \\
\hline \multirow[t]{3}{*}{3.20} & & & $f_{m}$ & 0.40 & 0.49 \\
\hline & & & $f_{w}$ & 0.40 & 0.48 \\
\hline & & & $-\frac{\Delta S}{k N}$ & 0.48 & 0.41 \\
\hline \multirow[t]{3}{*}{3.25} & & & $f_{m}$ & 0.56 & 0.59 \\
\hline & & & $f_{w}$ & 0.54 & 0.59 \\
\hline & & & $-\frac{\Delta S}{k N}$ & 0.37 & 0.33 \\
\hline \multirow[t]{12}{*}{3.30} & & & $f_{m}$ & 0.65 & 0.67 \\
\hline & & & $f_{w}$ & 0.63 & 0.66 \\
\hline & & & $-\frac{\Delta S}{k N}$ & 0.39 & 0.27 \\
\hline & 1.75 & 1.75 & $f_{m}$ & 0.33 & 0.44 \\
\hline & & & $f_{w}$ & 0.32 & 0.42 \\
\hline & & & $-\frac{\Delta S}{k N}$ & 0.55 & 0.46 \\
\hline & 1.80 & 1.80 & $f_{m}$ & 0.43 & 0.54 \\
\hline & & & $f_{w}$ & 0.44 & 0.51 \\
\hline & & & $-\frac{\Delta S}{k N}$ & 0.45 & 0.39 \\
\hline & 1.85 & 1.85 & $f_{m}$ & 0.52 & 0.65 \\
\hline & & & $f_{w}$ & 0.54 & 0.60 \\
\hline & & & $-\frac{\Delta S}{k N}$ & 0.37 & 0.31 \\
\hline \multirow[t]{3}{*}{3.10} & 1.70 & 1.70 & $f_{m}$ & 0.35 & 0.52 \\
\hline & & & $f_{w}$ & 0.34 & 0.48 \\
\hline & & & $-\frac{\Delta S}{k N}$ & 0.53 & 0.41 \\
\hline \multirow[t]{3}{*}{3.10} & 1.75 & 1.75 & $f_{m}$ & 0.42 & 0.61 \\
\hline & & & $f_{w}$ & 0.41 & 0.59 \\
\hline & & & $-\frac{\Delta S}{k N}$ & 0.47 & 0.33 \\
\hline \multirow[t]{3}{*}{3.10} & 1.80 & 1.80 & $f_{m}$ & 0.51 & 0.70 \\
\hline & & & $f_{w}$ & 0.51 & 0.65 \\
\hline & & & $-\frac{\Delta S}{k N}$ & 0.39 & 0.27 \\
\hline
\end{tabular}

Table 8.5: Estimated surface fractions and modified excess entropy of methanol-water solutions, after correcting for the fraction of methanol $\left(f_{m}\right)$ and water $\left(f_{w}\right)$ molecules that occur in the interfacial regions of the respective clusters. 


\section{Chapter 9}

\section{Conclusions and Future Work}

\subsection{Conclusions}

A thorough investigation of methanol-water mixtures across the concentration range using neutron diffraction experiments combined with Empirical Structural Refinement Analysis (EPSR) has shown that these mixtures exhibit extended structures in solution despite the components being fully miscible in all proportions. In this system, there is a concentration region (methanol mole fraction between 0.27 and 0.54 ) where both methanol and water appear to form separate, percolating networks. Interestingly, it is within this concentration range where many transport properties and thermodynamic excess functions reach extremal values. Therefore, it may be that the observed concentration dependence of these functions in methanol-water solutions have a structural origin.

Studies of methanol-water mixtures across a wide concentration range as a function of temperature and pressure have revealed significantly enhanced segregation. This evolution toward greater molecular heterogeneity in the mixture accounts for the observed changes in the water-water radial distribution function and there are indications also of a change in the topology of the water clusters. The observed behaviour is consistent 
with an approach to an upper critical solution point. Such a point would appear to be 'hidden' below the freezing line, thereby precluding observation of the two-fluid region.

The negative excess entropy of mixing characteristic of aqueous lower alcohols can be understood quantitatively in terms of molecular-scale segregation of the components. A simple model can be applied and allows understanding of the behaviour of methanol-water solutions under extreme conditions. Using molecular clustering data from neutron diffraction the model obviates the need to invoke other restructuring concepts which, though well-known, are unsupported by recent experiments.

\subsection{Future Work}

Aqueous alcohols systems have proved very resourceful model systems for exploring the complexities of the hydrophobic effect, hydrogen bonding, molecular structure and dynamics. It is now essential that the next step is taken and that the techniques and interrogation methods developed here are used to further explore these important processes. Therefore, the next step could be to extend the study of the lower alcohols to that of small peptide fragments. Protein molecules consist of one, or a small number, of polypeptide chains each of which is a linear polymer of up to several hundred amino acids. The amino acids in the chain are joined together by a peptide bond. A peptide bond involves the linkage between the amino group of one amino acid and the carboxyl group of another amino acid.

N-methylacetamide (NMA) is a minimal model of peptide linkage and has the chemical formula $\mathrm{C}_{3} \mathrm{H}_{7} \mathrm{NO}$. This molecule has been the focus of numerous computational investigations to understand the hydration structure and examine local and medium range structure of NMA. However, no real experimental structural information exists for this molecule. In order to investigate computer simulation predictions, and to fully 
appreciate the subtleties of this molecule, real experimental tests on NMA are essential. A neutron diffraction study on this important model peptide fragment, similar to the one described in this thesis, could provide unambiguous data to provide further insight into the complexities of biologically complex macromolecules. 


\section{Appendix A}

\section{Published Papers}

- L.Dougan, A.K.Soper, J.Crain and J.L.Finney, Mixing in aqueous methanol at extreme conditions: Excess entropy from a simple clustering model J. Phys. Chem., submitted, 2005.

- A.K.Soper, L.Dougan, J.Crain and J.L.Finney, Excess entropy in alcohol-water solutions: a simple clustering explanation J. Phys. Chem. B, accepted, 2005.

- L.Dougan, R.Hargreaves, S.P.Bates, J.L.Finney, V.Réat, A.K.Soper and J.Crain, Segregation in aqueous methanol enhanced by cooling and compression. $J$. Chem. Phys., 122, 174514-17421, 2005.

- L.Dougan, S.P.Bates, R.Hargreaves, J.P.Fox, J.Crain, J.L.Finney, V.Réat and A.K.Soper. Methanol-water solutions: A bi-percolating liquid mixture. J. Chem. Phys. , 121, 6456-6462, 2004.

- L.Dougan, J.Crain, H.Vass and S.W.Magennis. Probing the liquid state structure and dynamics of aqueous solutions using fluorescence spectroscopy J. Fluorescence, 14, 91-97 2004.

- J.Crain, S.P.Bates, S.W.Magennis and L.Dougan. Recent research developments 


\section{Appendix B}

\section{Intermolecular Weights}




\begin{tabular}{|c|ccccc|}
\hline HH Weights & M & & & & \\
\hline M & 1 & & & & \\
& & & & & \\
\hline XH Weights & C & O & H & Ow & Hw \\
\hline M & 0.09376 & 0.08187 & 0.09411 & 0.22135 & 0.50891 \\
& & & & & \\
\hline XX Weights & C & O & H & Ow & Hw \\
\hline C & 0.00879 & & & & \\
O & 0.01535 & 0.00670 & & & \\
H & 0.01765 & 0.01541 & 0.00886 & & \\
Ow & 0.04151 & 0.03624 & 0.04166 & 0.04899 & \\
Hw & 0.09543 & 0.08333 & 0.09579 & 0.22529 & 0.25899 \\
\hline
\end{tabular}

Table B.1: Intermolecular weights for $\mathrm{x}=0.27$ mole fraction methanol water mixture where substitutions are made on the methyl hydrogens. The atom labels $\mathrm{M}, \mathrm{C}, \mathrm{O}$ and $\mathrm{H}$ refer to methanol methyl hydrogen, carbon, oxygen and hydroxyl hydrogen respectively. The atom labels $O_{W}$ and $H_{W}$ refer to water oxygen and hydrogen respectively. In the weights notation $\mathrm{H}$ corresponds to the labelled hydrogen and $\mathrm{X}$ corresponds to the remaining unlabelled atoms. 


\begin{tabular}{|c|cccc|}
\hline HH Weights & H & Hw & & \\
\hline H & 0.02434 & & & \\
Hw & 0.26342 & 0.71222 & & \\
& & & & \\
\hline XH Weights & C & O & M & Ow \\
\hline H & 0.02154 & 0.01881 & 0.06487 & 0.05085 \\
Hw & 0.11648 & 0.10171 & 0.35076 & 0.27498 \\
& & & & \\
\hline XX Weights & C & O & M & Ow \\
\hline C & 0.01905 & & & \\
O & 0.03327 & 0.01452 & & \\
M & 0.11473 & 0.10018 & 0.17274 & \\
Ow & 0.08995 & 0.07854 & 0.27085 & 0.10617 \\
\hline
\end{tabular}

Table B.2: Intermolecular weights for $\mathrm{x}=0.27$ mole fraction methanol water mixture where substitutions are made on the methanol hydroxyl hydrogens and water hydroxyl hydrogens. The atom labels $\mathrm{M}, \mathrm{C}, \mathrm{O}$ and $\mathrm{H}$ refer to methanol methyl hydrogen, carbon, oxygen and hydroxyl hydrogen respectively. The atom labels $O_{W}$ and $H_{W}$ refer to water oxygen and hydrogen respectively. In the weights notation $\mathrm{H}$ corresponds to the labelled hydrogen and $\mathrm{X}$ corresponds to the remaining unlabelled atoms. 


\begin{tabular}{|c|ccc|}
\hline HH Weights & M & H & Hw \\
\hline M & 0.10170 & & \\
H & 0.06780 & 0.01130 & \\
Hw & 0.36661 & 0.12220 & 0.33040 \\
& & & \\
\hline XH Weights & C & O & Ow \\
\hline M & 0.07532 & 0.06577 & 0.17781 \\
H & 0.02512 & 0.02192 & 0.05927 \\
Hw & 0.13576 & 0.11854 & 0.32050 \\
& & & \\
\hline XX Weights & C & O & Ow \\
\hline C & 0.05578 & & \\
O & 0.09742 & 0.04253 & \\
Ow & 0.26339 & 0.22998 & 0.31090 \\
\hline
\end{tabular}

Table B.3: Intermolecular weights for $\mathrm{x}=0.27$ mole fraction methanol water mixture where substitutions are made on all the hydrogens (methyl hydrogens, methanol hydroxyl hydrogens and water hydroxyl hydrogens). The atom labels $\mathrm{M}, \mathrm{C}, \mathrm{O}$ and $\mathrm{H}$ refer to methanol methyl hydrogen, carbon, oxygen and hydroxyl hydrogen respectively. The atom labels $O_{W}$ and $H_{W}$ refer to water oxygen and hydrogen respectively. In the weights notation $\mathrm{H}$ corresponds to the labelled hydrogen and $\mathrm{X}$ corresponds to the remaining unlabelled atoms. 


\begin{tabular}{|c|c|c|c|c|c|c|}
\hline 14394tot1 & $\mathrm{C}$ & $\mathrm{O}$ & M & $\mathrm{H}$ & $O_{W}$ & $H_{W}$ \\
\hline $\mathrm{C}$ & 0.00603 & & & & & \\
\hline $\mathrm{O}$ & 0.01054 & 0.00460 & & & & \\
\hline M & 0.03634 & 0.03173 & 0.05472 & & & \\
\hline $\mathrm{H}$ & 0.01211 & 0.01058 & 0.03648 & 0.00608 & & \\
\hline$O_{W}$ & 0.00898 & 0.00784 & 0.02703 & 0.00901 & 0.00334 & \\
\hline$H_{W}$ & 0.02064 & 0.01802 & 0.06215 & 0.02072 & 0.01535 & 0.01765 \\
\hline 14394tot2 & $\mathrm{C}$ & $\mathrm{O}$ & M & $\mathrm{H}$ & $O_{W}$ & $H_{W}$ \\
\hline $\mathrm{C}$ & 0.00603 & & & & & \\
\hline $\mathrm{O}$ & 0.01054 & 0.00460 & & & & \\
\hline M & -0.02037 & -0.01779 & 0.01719 & & & \\
\hline $\mathrm{H}$ & 0.01211 & 0.01058 & -0.02045 & 0.00608 & & \\
\hline$O_{W}$ & 0.00898 & 0.00784 & -0.02703 & 0.00901 & 0.00334 & \\
\hline$H_{W}$ & 0.02064 & 0.01802 & -0.06215 & 0.02072 & 0.01535 & 0.01765 \\
\hline 14394tot3 & $\mathrm{C}$ & $\mathrm{O}$ & $\mathrm{M}$ & $\mathrm{H}$ & $O_{W}$ & $H_{W}$ \\
\hline $\mathrm{C}$ & 0.00603 & & & & & \\
\hline $\mathrm{O}$ & 0.01054 & 0.00460 & & & & \\
\hline $\mathrm{M}$ & 0.00799 & 0.00697 & 0.00264 & & & \\
\hline $\mathrm{H}$ & 0.01211 & 0.01058 & 0.00802 & 0.00608 & & \\
\hline$O_{W}$ & 0.00898 & 0.00784 & 0.00594 & 0.00901 & 0.00334 & \\
\hline$H_{W}$ & 0.02064 & 0.01802 & 0.01366 & 0.02072 & 0.01535 & 0.01765 \\
\hline
\end{tabular}

Table B.4: Intermolecular weights for methanol-water $x=0.54$ mole fraction mixture where substitutions are made on the methanol methyl hydrogens (note. it is only the $\mathrm{M}$ correlation weights which change between different weights files) The atom labels $\mathrm{M}, \mathrm{C}, \mathrm{O}$ and $\mathrm{H}$ refer to methanol methyl hydrogen, carbon, oxygen and hydroxyl hydrogen respectively. The atom labels $O_{W}$ and $H_{W}$ indicate water oxygen and water hydrogen. 


\begin{tabular}{|c|c|c|c|c|c|c|}
\hline 14417tot1 & $\mathrm{C}$ & $\mathrm{O}$ & $\mathrm{M}$ & $\mathrm{H}$ & $O_{W}$ & $H_{W}$ \\
\hline $\mathrm{C}$ & 0.00603 & & & & & \\
\hline $\mathrm{O}$ & 0.01054 & 0.00460 & & & & \\
\hline M & 0.03634 & 0.03173 & 0.05472 & & & \\
\hline $\mathrm{H}$ & 0.01211 & 0.01058 & 0.03648 & 0.00608 & & \\
\hline$O_{W}$ & 0.00898 & 0.00784 & 0.02703 & 0.00901 & 0.00334 & \\
\hline$H_{W}$ & 0.02064 & 0.01802 & 0.06215 & 0.02072 & 0.01535 & 0.01765 \\
\hline 14417 tot 2 & $\mathrm{C}$ & $\mathrm{O}$ & M & $\mathrm{H}$ & $O_{W}$ & $H_{W}$ \\
\hline $\mathrm{C}$ & 0.00603 & & & & & \\
\hline $\mathrm{O}$ & 0.01054 & 0.00460 & & & & \\
\hline M & 0.03634 & 0.03173 & 0.05472 & & & \\
\hline $\mathrm{H}$ & -0.00679 & -0.00593 & -0.02045 & 0.00191 & & \\
\hline$O_{W}$ & 0.00898 & 0.00784 & 0.02703 & -00505 & 0.00334 & \\
\hline$H_{W}$ & 0.02064 & 0.01802 & 0.06215 & 0.00651 & 0.01535 & 0.01765 \\
\hline 14417 tot 3 & $\mathrm{C}$ & $\mathrm{O}$ & M & $\mathrm{H}$ & $O_{W}$ & $H_{W}$ \\
\hline $\mathrm{C}$ & 0.00603 & & & & & \\
\hline $\mathrm{O}$ & 0.01054 & 0.00460 & & & & \\
\hline M & 0.03634 & 0.03173 & 0.05472 & & & \\
\hline $\mathrm{H}$ & 0.00266 & 0.00232 & 0.00802 & 0.00029 & & \\
\hline$O_{W}$ & 0.00898 & 0.00784 & 0.02703 & 0.00198 & 0.00334 & \\
\hline$H_{W}$ & 0.02064 & 0.01802 & 0.06215 & 0.00100 & 0.01535 & 0.01765 \\
\hline
\end{tabular}

Table B.5: Intermolecular weights for methanol-water $x=0.54$ mole fraction mixture where substitutions are made on methanol hydroxyl hydrogens and water hydroxyl hydrogens (note. it is only the $\mathrm{H}$ correlation weights which change between different weights files) The atom labels $\mathrm{M}, \mathrm{C}, \mathrm{O}$ and $\mathrm{H}$ refer to methanol methyl hydrogen, carbon, oxygen and hydroxyl hydrogen respectively. The atom labels $O_{W}$ and $H_{W}$ indicate water oxygen and water hydrogen. 


\begin{tabular}{|c|c|c|c|c|c|c|}
\hline 14439tot 1 & $\mathrm{C}$ & $\mathrm{O}$ & M & $\mathrm{H}$ & $O_{W}$ & $H_{W}$ \\
\hline $\mathrm{C}$ & 0.00603 & & & & & \\
\hline $\mathrm{O}$ & 0.01054 & 0.00460 & & & & \\
\hline M & 0.03634 & 0.03173 & 0.05472 & & & \\
\hline $\mathrm{H}$ & 0.01211 & 0.01058 & 0.03648 & 0.00608 & & \\
\hline$O_{W}$ & 0.00898 & 0.00784 & 0.02703 & 0.00901 & 0.00334 & \\
\hline$H_{W}$ & 0.02064 & 0.01802 & 0.06215 & 0.02072 & 0.01535 & 0.01765 \\
\hline 14439tot2 & $\mathrm{C}$ & $\mathrm{O}$ & $\mathrm{M}$ & $\mathrm{H}$ & $O_{W}$ & $H_{W}$ \\
\hline $\mathrm{C}$ & 0.00603 & & & & & \\
\hline $\mathrm{O}$ & 0.01054 & 0.00460 & & & & \\
\hline M & -0.0204 & -0.01779 & 0.01719 & & & \\
\hline $\mathrm{H}$ & -0.00679 & -0.00593 & 0.01146 & 0.00191 & & \\
\hline$O_{W}$ & 0.00898 & 0.00784 & -0.1515 & -0.00505 & 0.00334 & \\
\hline$H_{W}$ & 0.02064 & 0.01802 & 0.0195 & 0.00651 & 0.01535 & 0.01765 \\
\hline 14439tot3 & $\mathrm{C}$ & $\mathrm{O}$ & M & $\mathrm{H}$ & $O_{W}$ & $H_{W}$ \\
\hline $\mathrm{C}$ & 0.00603 & & & & & \\
\hline $\mathrm{O}$ & 0.01054 & 0.00460 & & & & \\
\hline M & 0.007986 & 0.00697 & 0.00264 & & & \\
\hline $\mathrm{H}$ & 0.00266 & 0.00232 & 0.00176 & 0.00029 & & \\
\hline$O_{W}$ & 0.00898 & 0.00784 & 0.00594 & 0.00198 & 0.00334 & \\
\hline$H_{W}$ & 0.02064 & 0.01802 & 0.00300 & 0.00100 & 0.01535 & 0.01765 \\
\hline
\end{tabular}

Table B.6: Intermolecular weights for methanol-water $x=0.54$ mole fraction mixture where substitutions are made on all hydrogens (note. it is only the $\mathrm{M}$ and $\mathrm{H}$ correlation weights which change between different weights files) The atom labels $\mathrm{M}, \mathrm{C}, \mathrm{O}$ and $\mathrm{H}$ refer to methanol methyl hydrogen, carbon, oxygen and hydroxyl hydrogen respectively. The atom labels $O_{W}$ and $H_{W}$ indicate water oxygen and water hydrogen. 


\begin{tabular}{|c|c|c|c|c|c|}
\hline HH Weights & $\mathrm{M}$ & & & & \\
\hline $\mathrm{M}$ & 1 & & & & \\
\hline XH Weights & $\mathrm{C}$ & $\mathrm{O}$ & $\mathrm{H}$ & $O_{W}$ & $H_{W}$ \\
\hline $\mathrm{M}$ & 0.17368 & 0.15165 & 0.17434 & 0.15165 & 0.34867 \\
\hline XX Weights & $\mathrm{C}$ & $\mathrm{O}$ & $\mathrm{H}$ & $O_{W}$ & $H_{W}$ \\
\hline $\mathrm{C}$ & 0.03017 & & & & \\
$\mathrm{O}$ & 0.05267 & 0.02300 & & & \\
$\mathrm{H}$ & 0.06056 & 0.05288 & 0.03039 & & \\
$O_{W}$ & 0.05267 & 0.04600 & 0.05288 & 0.02300 & \\
$H_{W}$ & 0.1211 & 0.10575 & 0.12157 & 0.10575 & 0.12157 \\
\hline
\end{tabular}

Table B.7: Intermolecular weights for the 50:50 methanol-water mixture where substitutions are made on the methyl hydrogens. The atom labels $\mathrm{M}, \mathrm{C}, \mathrm{O}$ and $\mathrm{H}$ refer to methanol methyl hydrogen, carbon, oxygen and hydroxyl hydrogen respectively. The atom labels $O_{W}$ and $H_{W}$ indicate water oxygen and water hydrogen. 


\begin{tabular}{|c|c|c|c|c|}
\hline HH Weights & $\mathrm{H}$ & $H_{W}$ & & \\
\hline $\begin{array}{c}H_{W} \\
\mathrm{H}\end{array}$ & & 0.44444 & & \\
\hline XH Weights & $\mathrm{H}$ & $H_{W}$ & $O_{W}$ & \\
\hline $\mathrm{C}$ & 0.05789 & 0.11579 & & \\
$\mathrm{O}$ & 0.05055 & 0.10110 & & \\
$\mathrm{M}$ & 0.17434 & 0.34867 & & \\
$\mathrm{H}$ & & & 0.05055 & \\
$H_{W}$ & & & 0.10110 & \\
\hline XX Weights & $\mathrm{C}$ & $\mathrm{O}$ & $\mathrm{M}$ & $O_{W}$ \\
\hline $\mathrm{C}$ & 0.03017 & & & \\
$\mathrm{O}$ & 0.05267 & 0.02300 & & \\
$\mathrm{M}$ & 0.18167 & 0.15863 & 0.27354 & \\
$O_{W}$ & 0.05267 & 0.04600 & 0.15863 & 0.02300 \\
\hline
\end{tabular}

Table B.8: Intermolecular weights for the 50:50 methanol-water mixture where substitutions are made on the methanol hydroxyl hydrogen and the water hydroxyl hydrogens. The atom labels $\mathrm{M}, \mathrm{C}, \mathrm{O}$ and $\mathrm{H}$ refer to methanol methyl hydrogen, carbon, oxygen and hydroxyl hydrogen respectively. The atom labels $O_{W}$ and $H_{W}$ indicate water oxygen and water hydrogen. 


\section{Appendix C}

\section{Coordination Numbers}




\begin{tabular}{|c|c|c|c|l|}
\hline Correlation & $\begin{array}{c}\text { Atomic density } \\
\rho\end{array}$ & $\begin{array}{c}R_{\min } \\
\AA\end{array}$ & $\begin{array}{c}R_{\max } \\
\AA\end{array}$ & $\begin{array}{l}\text { Coordination No. } \\
\text { at } 298 \mathrm{~K} \text { (atoms) }\end{array}$ \\
\hline \hline$C C$ & 0.0147 & 3.0 & 5.66 & $10.8 \pm 0.28$ \\
\hline$C O$ & 0.0147 & 2.5 & 4.45 & $4.84 \pm 0.13$ \\
\hline$C O$ & 0.0147 & 4.45 & 6.02 & $7.61 \pm 0.17$ \\
\hline$O O$ & 0.0147 & 2.0 & 3.48 & $1.92 \pm 0.12$ \\
\hline$O H$ & 0.0147 & 1.0 & 2.62 & $0.92 \pm 0.06$ \\
\hline$H H$ & 0.0147 & 1.5 & 3.28 & $2.1 \pm 0.1$ \\
\hline
\end{tabular}

Table C.1: Coordination numbers for pure methanol obtained from integration of the peaks observed in the partial pair distribution functions at 298K obtained from the EPSR analysis[106].

\begin{tabular}{|c|c|c|c|l|}
\hline Correlation & $\begin{array}{c}\text { Atomic density } \\
\rho\end{array}$ & $\begin{array}{c}R_{\min } \\
\AA\end{array}$ & $\begin{array}{c}R_{\max } \\
\AA\end{array}$ & $\begin{array}{l}\text { Coordination No. } \\
\text { at } 298 \mathrm{~K} \text { (atoms) }\end{array}$ \\
\hline \hline$O_{W} O_{W}$ & 0.033 & 2.0 & 3.4 & $4.6 \pm 0.1$ \\
\hline$O_{W} O_{W}$ & 0.033 & 3.4 & 5.58 & $18.6 \pm 0.3$ \\
\hline$O_{W} H_{W}$ & 0.033 & 1.0 & 2.4 & $1.8 \pm 0.06$ \\
\hline
\end{tabular}

Table C.2: Coordination numbers for pure water obtained from the integration of the peaks observed in the partial pair distributions functions at 298K obtained from the EPSR analysis[126] 


\begin{tabular}{|c|c|c|c|c|c|}
\hline Correlation & $\begin{array}{c}\text { Temp. } \\
/ \mathrm{K}\end{array}$ & $\begin{array}{c}R_{\min } / \AA \\
/ \AA\end{array}$ & $\begin{array}{c}R_{\max } \\
/ \AA\end{array}$ & Coordination No. & error \\
\hline $\mathrm{CC}$ & 298 & 3.00 & 5.66 & 4.96 & 0.51 \\
\hline $\mathrm{CC}$ & 238 & 3.00 & 5.66 & 4.90 & 0.35 \\
\hline $\mathrm{CO}$ & 298 & 2.00 & 4.45 & 2.74 & 0.38 \\
\hline $\mathrm{CO}$ & 238 & 2.00 & 4.45 & 2.89 & 0.40 \\
\hline$C O_{W}$ & 298 & 2.00 & 5.04 & 8.47 & 0.54 \\
\hline$C O_{W}$ & 238 & 2.00 & 5.04 & 8.66 & 0.77 \\
\hline $\mathrm{OO}$ & 298 & 2.00 & 3.48 & 1.55 & 0.14 \\
\hline $\mathrm{OO}$ & 238 & 2.00 & 3.48 & 1.79 & 0.11 \\
\hline $\mathrm{OH}$ & 298 & 1.50 & 2.50 & 0.64 & 0.14 \\
\hline $\mathrm{OH}$ & 238 & 1.50 & 2.50 & 0.76 & 0.15 \\
\hline$O O_{W}$ & 298 & 2.00 & 3.10 & 0.80 & 0.08 \\
\hline$O O_{W}$ & 238 & 2.00 & 3.10 & 0.64 & 0.07 \\
\hline$O H_{W}$ & 298 & 1.50 & 2.50 & 0.66 & 0.02 \\
\hline$O H_{W}$ & 238 & 1.50 & 2.50 & 0.59 & 0.02 \\
\hline $\mathrm{HH}$ & 298 & 1.50 & 3.10 & 1.70 & 0.16 \\
\hline $\mathrm{HH}$ & 238 & 1.50 & 3.10 & 2.05 & 0.19 \\
\hline$H O_{W}$ & 298 & 1.50 & 2.50 & 0.22 & 0.01 \\
\hline$H O_{W}$ & 238 & 1.50 & 2.50 & 0.11 & 0.01 \\
\hline$H H_{W}$ & 298 & 1.50 & 3.10 & 1.63 & 0.19 \\
\hline$H H_{W}$ & 238 & 1.50 & 3.10 & 1.24 & 0.17 \\
\hline$O_{W} O_{W}$ & 298 & 2.00 & 3.40 & 3.66 & 0.16 \\
\hline$O_{W} O_{W}$ & 238 & 2.00 & 3.40 & 3.54 & 0.18 \\
\hline$O_{W} H_{W}$ & 298 & 1.50 & 2.40 & 1.48 & 0.19 \\
\hline$O_{W} H_{W}$ & 238 & 1.50 & 2.40 & 1.55 & 0.14 \\
\hline$H_{W} H_{W}$ & 298 & 1.50 & 3.00 & 4.04 & 0.25 \\
\hline$H_{W} H_{W}$ & 238 & 1.50 & 3.00 & 4.04 & 0.26 \\
\hline$O_{W} O_{W}$ & 298 & 3.40 & 5.58 & 10.80 & 0.34 \\
\hline$O_{W} O_{W}$ & 238 & 3.40 & 5.58 & 11.04 & 0.36 \\
\hline
\end{tabular}

Table C.3: Coordination numbers for methanol -water solution mole fraction $x=0.27$ at (i) 298 $\mathrm{K}$ and (ii) $238 \mathrm{~K}$ 


\begin{tabular}{|c|c|c|c|c|c|}
\hline Correlation & $\begin{array}{c}\text { Temp. } \\
\text { /K }\end{array}$ & $\begin{array}{c}R_{\min } \\
/ \AA\end{array}$ & $\begin{array}{c}R_{\max } \\
/ \AA\end{array}$ & Coordination No. & Error \\
\hline $\mathrm{CC}$ & 298 & 3.00 & 5.66 & 7.86 & 0.35 \\
\hline $\mathrm{CC}$ & 260 & 3.00 & 5.66 & 8.04 & 0.30 \\
\hline $\mathrm{CC}$ & 190 & 3.00 & 5.66 & 8.17 & 0.38 \\
\hline $\mathrm{CO}$ & 298 & 2.00 & 4.45 & 3.15 & 0.27 \\
\hline $\mathrm{CO}$ & 260 & 2.00 & 4.45 & 3.23 & 0.21 \\
\hline $\mathrm{CO}$ & 190 & 2.00 & 4.45 & 3.73 & 0.27 \\
\hline$C O_{W}$ & 298 & 2.50 & 5.04 & 4.96 & 0.16 \\
\hline$C O_{W}$ & 260 & 2.50 & 5.04 & 5.12 & 0.15 \\
\hline$C O_{W}$ & 190 & 2.50 & 5.04 & 5.30 & 0.19 \\
\hline $\mathrm{OO}$ & 298 & 2.00 & 3.48 & 1.29 & 0.21 \\
\hline $\mathrm{OO}$ & 260 & 2.00 & 3.48 & 1.36 & 0.19 \\
\hline $\mathrm{OO}$ & 190 & 2.00 & 3.48 & 1.49 & 0.20 \\
\hline $\mathrm{OH}$ & 298 & 1.50 & 2.50 & 0.47 & 0.02 \\
\hline $\mathrm{OH}$ & 260 & 1.50 & 2.50 & 0.55 & 0.03 \\
\hline $\mathrm{OH}$ & 190 & 1.50 & 2.50 & 0.63 & 0.05 \\
\hline$O O_{W}$ & 298 & 2.00 & 3.10 & 1.30 & 0.19 \\
\hline$O O_{W}$ & 260 & 2.00 & 3.10 & 1.18 & 0.22 \\
\hline$O O_{W}$ & 190 & 2.00 & 3.10 & 0.98 & 0.20 \\
\hline$O H_{W}$ & 298 & 1.50 & 2.50 & 1.00 & 0.21 \\
\hline$O H_{W}$ & 260 & 1.50 & 2.50 & 0.84 & 0.19 \\
\hline$O H_{W}$ & 190 & 1.50 & 2.50 & 0.72 & 0.21 \\
\hline $\mathrm{HH}$ & 298 & 1.50 & 3.10 & 1.33 & 0.14 \\
\hline $\mathrm{HH}$ & 260 & 1.50 & 3.10 & 1.50 & 0.13 \\
\hline $\mathrm{HH}$ & 190 & 1.50 & 3.10 & 1.69 & 0.11 \\
\hline$H O_{W}$ & 298 & 1.50 & 2.50 & 0.51 & 0.01 \\
\hline$H O_{W}$ & 260 & 1.50 & 2.50 & 0.38 & 0.01 \\
\hline$H O_{W}$ & 190 & 1.50 & 2.50 & 0.27 & 0.02 \\
\hline$H H_{W}$ & 298 & 1.50 & 3.10 & 2.12 & 0.18 \\
\hline$H H_{W}$ & 260 & 1.50 & 3.10 & 2.16 & 0.21 \\
\hline$H H_{W}$ & 190 & 1.50 & 3.10 & 1.68 & 0.17 \\
\hline
\end{tabular}




\begin{tabular}{|c|c|c|c|c|c|}
\hline Correlation & $\begin{array}{c}\text { Temp. } \\
/ \mathrm{K}\end{array}$ & $\begin{array}{c}R_{\min } / \AA \\
/ \AA\end{array}$ & $\begin{array}{c}R_{\max } \\
/ \AA\end{array}$ & Coordination No. & Error \\
\hline$O_{W} O_{W}$ & 298 & 2.00 & 3.40 & 1.85 & 0.14 \\
\hline$O_{W} O_{W}$ & 260 & 2.00 & 3.40 & 1.91 & 0.16 \\
\hline$O_{W} O_{W}$ & 190 & 2.00 & 3.40 & 2.07 & 0.15 \\
\hline$O_{W} H_{W}$ & 298 & 1.50 & 2.40 & 0.87 & 0.03 \\
\hline$O_{W} H_{W}$ & 260 & 1.50 & 2.40 & 0.83 & 0.02 \\
\hline$O_{W} H_{W}$ & 190 & 1.50 & 2.40 & 0.93 & 0.02 \\
\hline$H_{W} H_{W}$ & 298 & 1.50 & 3.00 & 2.01 & 0.14 \\
\hline$H_{W} H_{W}$ & 260 & 1.50 & 3.00 & 2.17 & 0.15 \\
\hline$H_{W} H_{W}$ & 190 & 1.50 & 3.00 & 2.38 & 0.12 \\
\hline$O_{W} O_{W}$ & 298 & 3.40 & 5.58 & 5.36 & 0.25 \\
\hline$O_{W} O_{W}$ & 260 & 3.40 & 5.58 & 5.32 & 0.23 \\
\hline$O_{W} O_{W}$ & 190 & 3.40 & 5.58 & 5.04 & 0.27 \\
\hline
\end{tabular}

Table C.5: Coordination numbers for methanol -water solution mole fraction $x=0.54$ at (i)298 $\mathrm{K}$ and (ii) $260 \mathrm{~K}$ and (iii) $190 \mathrm{~K}$ 


\begin{tabular}{|c|c|c|c|c|c|}
\hline Correlation & $\begin{array}{c}\text { Pressure } \\
\text { /kbar }\end{array}$ & $\begin{array}{c}R_{\min } \\
/ \AA\end{array}$ & $\begin{array}{c}R_{\max } \\
/ \AA\end{array}$ & Coordination No. & Error \\
\hline $\mathrm{CC}$ & $a m b$ & 3.00 & 5.66 & 8.19 & 0.31 \\
\hline $\mathrm{CC}$ & $2 \mathrm{kbar}$ & 3.00 & 5.66 & 9.01 & 0.39 \\
\hline $\mathrm{CO}$ & $\mathrm{amb}$ & 2.00 & 4.45 & 3.64 & 0.25 \\
\hline $\mathrm{CO}$ & $2 \mathrm{kbar}$ & 2.00 & 4.45 & 3.97 & 0.29 \\
\hline$C O_{W}$ & $\mathrm{amb}$ & 2.00 & 5.04 & 5.84 & 0.26 \\
\hline$C O_{W}$ & $2 \mathrm{kbar}$ & 2.00 & 5.04 & 6.54 & 0.23 \\
\hline $\mathrm{OO}$ & $\mathrm{amb}$ & 2.00 & 3.48 & 1.02 & 0.14 \\
\hline $\mathrm{OO}$ & $2 \mathrm{kbar}$ & 2.00 & 3.48 & 1.08 & 0.17 \\
\hline $\mathrm{OH}$ & $\mathrm{amb}$ & 1.50 & 2.50 & 0.45 & 0.03 \\
\hline $\mathrm{OH}$ & $2 \mathrm{kbar}$ & 1.50 & 2.50 & 0.44 & 0.05 \\
\hline$O O_{W}$ & $\mathrm{amb}$ & 2.00 & 3.10 & 1.38 & 0.18 \\
\hline$O O_{W}$ & $2 \mathrm{kbar}$ & 2.00 & 3.10 & 1.54 & 0.19 \\
\hline$O H_{W}$ & $a m b$ & 1.50 & 2.50 & 0.99 & 0.05 \\
\hline$O H_{W}$ & $2 \mathrm{kbar}$ & 1.50 & 2.50 & 1.07 & 0.09 \\
\hline $\mathrm{HH}$ & $a m b$ & 1.50 & 3.10 & 1.06 & 0.11 \\
\hline $\mathrm{HH}$ & $2 \mathrm{kbar}$ & 1.50 & 3.10 & 1.18 & 0.09 \\
\hline$H O_{W}$ & $a m b$ & 1.50 & 2.50 & 0.52 & 0.01 \\
\hline$H O_{W}$ & $2 \mathrm{kbar}$ & 1.50 & 2.50 & 0.54 & 0.01 \\
\hline$H H_{W}$ & $a m b$ & 1.50 & 3.10 & 2.43 & 0.19 \\
\hline$H H_{W}$ & $2 \mathrm{kbar}$ & 1.50 & 3.10 & 2.70 & 0.18 \\
\hline$O_{W} O_{W}$ & $\mathrm{amb}$ & 2.00 & 3.400 & 2.05 & 0.16 \\
\hline$O_{W} O_{W}$ & $2 \mathrm{kbar}$ & 2.00 & 3.40 & 2.10 & 0.19 \\
\hline$O_{W} H_{W}$ & $a m b$ & 1.50 & 2.40 & 0.90 & 0.02 \\
\hline$O_{W} H_{W}$ & $2 \mathrm{kbar}$ & 1.50 & 2.40 & 0.85 & 0.01 \\
\hline$H_{W} H_{W}$ & $a m b$ & 1.50 & 3.00 & 2.16 & 0.19 \\
\hline$H_{W} H_{W}$ & $2 \mathrm{kbar}$ & 1.50 & 3.00 & 2.19 & 0.21 \\
\hline$O_{W} O_{W}$ & $a m b$ & 3.40 & 5.58 & 6.15 & 0.38 \\
\hline$O_{W} O_{W}$ & $2 \mathrm{kbar}$ & 3.40 & 5.58 & 6.79 & 0.43 \\
\hline
\end{tabular}

Table C.6: Coordination numbers for methanol -water solution mole fraction $x=0.50$ at (i) 200 $\mathrm{K}$ and ambient pressure and (ii) $200 \mathrm{~K}$ and $2 \mathrm{kbar}$ 


\section{Bibliography}

[1] P. Ball. $\mathrm{H}_{2} \mathrm{O}$ : A Biography of Water. Pheonix Paperback, UK, (1999).

[2] J.A. Rupley and G. Careri. Protein hydration and function. Adv. Protein Chem., 41:37, (1991).

[3] J.A. Kornblatt and M.J. Kornblatt. Water as it applies to the function of enzymes. Int. Rev. Cytol., 215:49, (2002).

[4] Y. Pocker. Water in enzyme reactions:biophysical aspects of hydrationdehydration processes. Cell. Mol. Life Sci., 57:1008, (2000).

[5] S. Zhang. Emerging biological materials through molecular self-assembly. Biotechnology Advances, 20:321, 2002.

[6] S. Garde, G. Hummer, A.E. Garcia, L. Pratt, and M.E. Paulaitis. Hydrophobic hydration: Inhomogeneous water structure near non-polar molecular solutes. Phys. Rev. E, 53:4310, 1996.

[7] L. Pratt and A. Pohorille. Hydrophobic effects and modeling of biophysical aqueous solution interfaces. Chem. Rev, 102:2671, 2002.

[8] D. Svergun, S. Richard, M.H.J. Koch, Z. Sayers, S. Kuprin, and G. Zaccai. Protein hydration in solution: Experimental observation by x-ray and neutron scattering. Proc. Natl. Acad. Sci., 95:2267, 1998.

[9] M. Levitt and R. Sharon. Accurate simulation of protein dynamics in solution. Proc. Natl. Acad. Sci., 85:7557, 1988.

[10] H.S. Frank and M.W. Evans. Free volume and entropy in condensed systems. $J$. Chem. Phys., 13:507, 1945.

[11] Y.-K. Cheng and P. Rossky. Surface topography dependence of biomolecular hydrophobic hydration. Nature, 392:696, 1998. 
[12] S. Dixit, J. Crain, W.C.K. Poon, J.L. Finney, and A.K. Soper. Molecular segregation observed in a concentrated alcohol-water solution. Nature, 416:829, 2002.

[13] S. Dixit, A.K. Soper, J.L. Finney, and J. Crain. Water structure and solute association in dilute aqueous methanol. Europhys. Lett., 59:377, 2002.

[14] S. Dixit, W.C.K. Poon, and J. Crain. Hydration of methanol in aqueous solutions:a raman spectrscopy study. J. Phys. Condensed Matter, 12:L323, 2000.

[15] O. Collet. Warm and cold denaturation in the phase diagram of a protein lattice model. Europhys. Lett., 53:93, 2001.

[16] M. Peyrard. Glass transition in protein hydration water. Phys. Rev. E, 64:11109, 2001.

[17] G. Careri and G. Consolini. Dielectric 1/f noise of proton glass on a hydrated protein surface. Phys. Rev, E, 62:4454, 2001.

[18] K. Ito, C.T. Moynihan, and A. Angell. Thermodynamic determination of fragility in liquids and a fragile-to-strong liquid transition in water. Nature, 398:492, 1999.

[19] C. A. Angell et al. Liquid fragility and the glass transition in water and aqueous solutions. Chem. Rev, 102:2627, 2002.

[20] S. Sastry, P.G. Debenedetti, F. Sciortino, and H.E. Stanley. Singularity-free interpretation of the thermodynamics of supercooled water. Phys. Rev. E, 53:6144, 1996.

[21] T. Koop, B. Luo, A. Tsias, and T. Peter. Water activity as the determinant for homogeneous ice nucleation in aqueous solutions. Nature, 406:611, 2000.

[22] T. Chwartz. Chemistry in Context. Willian Brown Publishers, USA, (1991).

[23] C. Tanford. The hydrophobic Effect:Formation of Micelles and Biological Membranes. Krieger Publishing Company, USA, (1991).

[24] J. Israelachvili. Intermolecular and Surface Forces. Academic Press, USA, (1991).

[25] V. Chihaia, S. Adams, and W. F. Kuhs. Influence of water molecules arrangement on the structure and stability of 512 and 51262 buckyball water clusters. a theoretical study. Chem. Phys., 297:271, 2004.

[26] J.L. Finney and A.K. Soper. Hydration of methanol in aqueous solution. Phys. Rev. Lett., 71:4346, 1993. 
[27] D.T. Bowron, J. L. Finney, and A. K. Soper. Structural investigation of solutesolute interactions in aqueous solutions of tertiary butanol. J. Phys. Chem. B., 102:3551, 1998.

[28] J. Turner, A.K. Soper, and J.L. Finney. Water structure in aqueous solutions of tetraamethylammonium chloride. Molecular Physics, 77:411, (1992).

[29] J.Turner and A.K. Soper. The effect of apolar solutes on water structure-alcohols and tetramethylammonium ions. J. Chem. Phys., 101:6116, (1994).

[30] B. Guillot. A reappraisal of what we have learnt during three decades of computer simulations on water. Journal of Molecular Liquids, 101:219-260", 2002.

[31] T.M. Raschke, J. Tsai, and M. Levitt. Quantification of the hydrophobic interaction by simulations of the aggregation of small hydrophobic solutes in water. Proc. Natl. Acad. Sci. USA, 98(11):5965-5969, 2001.

[32] S. Okazaki, H. Touhara, and K. Nakanishi. Computer experiments of aqueous solutions. v. monte carlo calculation on the hydrophobic interaction in $5 \mathrm{~mol} \%$ methanol solution. J. Chem. Phys, 81(2):890-894, 1984.

[33] G. Bolis, C. Corongui, and E. Clementi. Methanol in water solution at 300k. Chem. Phys. Lett, 86(3):299, 1982.

[34] G. Palinkas, E. Hawlicka, and K. Heinzinger. Molecular dynamics simulations of water-methanol mixtures. Chem. Phys., 158:65-76, 1991.

[35] M. Ferrario, M. Haughney, I.R. McDonald, and M.L. Klein. Moleculardynamics simulation of aqueous mixtures: Methanol, acetone and ammonia. J. Chem. Phys., 93(7):5156-5166, 1990.

[36] H. Tanaka and K.E. Gubbins. Structure and thermodynamic properties of water-methanol mixtures: Role of the water-water interaction. J. Chem. Phys., 97(4):2626-2634, 1992.

[37] W.L. Jorgensen and J.D. Madura. Quantum and statistical studies of liquids. solvation and conformation of methanol in water. J. Am. Chem. Soc., 105(6):14071413, 1983.

[38] J. Fidler and P.M. Rodger. Solvation structure around aqueous alcohols. J. Phys. Chem. B, 103:7695-7703, 1999.

[39] E.C. Meng and P.A. Kollman. Molecular dynamics studies of the properties of water around simple organic solutes. J. Phys. Chem., 100:11460-11470, 1996.

[40] A. Laaksonen, P.G. Kusalik, and I.M. Svishchev. Three dimensional structure in water-methanol mixtures. J. Phys. Chem. A, 101:5910-5918, 1997. 
[41] T.S. van Erp and E.J. Meijer. Ab initio molecular dynamics study of aqueous solvation of ethanol and ethylene. J. Chem. Phys., 118(9):8831-8840, 2003.

[42] E. Ruckenstein, I. Shulgin, and J. Tilson. Treatment of dilute clusters of methanol and water by ab initio quantum mechanical calculations. J. Phys. Chem. A, 107:2289, 2003.

[43] J.R. Lakowicz. Principles of Fluorescence Spectroscopy. Plenum Press, New York, (1983).

[44] L. Stryer. The interaction of a naphthalene dye with apomyoglobin and apohemoglobin: A fluorescent probe of non-polar binding sites. J. Mol. Biol, 13:482, (1965).

[45] G.W. Robinson, R.J. Robbins, G.R. Fleming, J.M. Morris, A.E.W. Knight, and R.J.S. Morrison. Fluorescence probe molecule anilinonaphthalenesulfonic acid. American Chemical Society, 100:146, (1978).

[46] Edinburgh Instruments. F900 Software. EdInst, UK, 2001.

[47] N. Periasamy. Analysis of fluorescence decay by the nonlinear least-squares method. Biophys. J., 54:961, (1988).

[48] J.N. Demas. Excited State Lifetime Measurements. Academic Press, New York, (1983).

[49] J. Slavik. Anilinonaphthalene sulfonate as a probe of membrane composition and function. Biochem. Biophys. Acta., 694:1, (1982).

[50] E.M. Kosower. Intramolecular donor-acceptor systems. Acc. Chem. Res., 15:259, (1982).

[51] R.P. DeToma, J.H. Easter, and L. Brand. Dynamic interactions of the fluorescence probe with solvent environment. J. Am. Chem. Soc., 98:5001, (1976).

[52] E. Lippert. Spectroscopic determination of the dipole moments of aromatic hydrocarbons in the first excited singlet state. Z. Electrochem, 61:962, (1957).

[53] N.G. Bakhshiev. The spectroscopy of intermolecular interactions. Opt. Spectrosc.USSR, 16:821, (1964).

[54] T.W. Ebbesen and C.A. Ghiron. Role of specific solvation in the fluorescence sensitivity of 1,8-ans to water. J. Phys. Chem., 93:(7139), (1989).

[55] H. Nakamura and J. Tanaka. Temperature dependence of fluorescence lifetimes of 8-anilini-1naphthanlene sulfonate and solvent isotope effect. Chem. Phys. Lett., 78:57, (1981). 
[56] S.K. Chakrabarti and W.R. Ware. Nanosecond time-resolved emission spectroscopy of 1-anilino-8-naphthalene sulfonate. J. Chem. Phys., 55:5494, (1971).

[57] K. Itoh and T. Azumi. Shift of the emission band upon excitation at the long wavelength absorption edge. ii. importance of the soulte-solvent interaction and solvent reorientation relaxation process. J. Chem. Phys., 62:3431, (1975).

[58] T. Azumi, K. Itoh, and H. Shiraishi. Shift of the emission band upon excitation at the long wavelength absorption edge. iii. temperature dependence of the shift and correlation with the time dependent spectral shift. J. Chem. Phys., 65:2550, (1976).

[59] P.J. Sadkowski and G.R. Fleming. The influence of solvent-solute interaction on radiationless processes: Excited state dynamics of 1,8-anilinonapthalene sulphonate and related molecules. Chem. Phys., 54:79, (1980).

[60] W.R. Laws and L. Brand. Analysis of two-state excited state reactions. the fluorescence decay of 2 naphthol. J. Phys. Chem, 83:795, (1979).

[61] A.S.R. Koti and N. Periasamy. Solvent exchange in excited-state relaxation in mixed solvents. J. Fluorescence, 10:177, (2000).

[62] S.K. Pal, J. Peon, and A.H. Zewail. Ultrafast surface hydration dynamics and expression of protein functionality: alpha-chymotrypsin. Proc. Natl. Acad. Sci. USA, 99:15297, (2002).

[63] R.M. Stratt and M. Maroncelli. Nonreactive dynamics in solution:the emerging molecular view of solvation dynamics and vibrational relaxation. J. Phys. Chem., 100:12981, (1996).

[64] J. Gardecki and M. Marconcelli. Solvation and rotational dynamics in acetonitrile/proplylene carbonate mixture:a binary system for use in dynamical solvent effect studies. Chem. Phys. Lett., 301:571, (1999).

[65] J.A.B. Ferreira and S.M.B. Costa. Non-markovian effects in the radiationless decay of rhodamine $3 b+$ in water-ethanol mixtures. Phys. Chem. Chem. Phys., 5:1064, (2003).

[66] S.R. Meech and D. Phillips. Time-resolved fluorescence of pdimethylaminobenzonitrile in mixed solvents. J. Chem. Soc. Faraday Trans., 83:1941, (1987).

[67] T. Molotsky and D. Huppert. Site specific solvation dynamics of coumarin dyes in hexane-methanol mixtures. J. Phys. Chem. A, 107:2769, (2003). 
[68] D. Huppert, H. Kanety, and E.M. Kosower. Kinetic studies on intramolecular electron transfer in solution. Chem. Phys. Lett., 84:48, (1981).

[69] M.M.G. Krishna. Excited-state kinetics of the hydrophobic probe nile red in membranes and micelles. J. Phys. Chem. A, 103:3589, (1999).

[70] Z.R. Grabowski, K. Rotkiewicz, and W. Rettig. Structural changes accompanying intramolecular electron transfer: Focus on twisted intramolecular chargetransfer states and structures. Chem. Rev., 103:3899, (2003).

[71] R. F. Lama and B. C.-Y.Lu. Excess thermodynamic properties of aqueous alcohol solutions. J. Chem. Eng. Data, 10:216-219, 1965.

[72] H. Vass, D. Edington, and J. Crain. Optical spectroscopy of simple aqueous solutions under extreme conditions. J. Chem. Phys., 118:11066, (2003).

[73] R. Zana and M.J. Eliebari. Fluorescence probing investigation of selfassociation of alcohols in aqueous solutions. J. Phys. Chem., 97:11134, (1993).

[74] B. Kirchner, J. Stubbs, and D. Marx. Fast anomalous diffusion of small hydrophobic species in water. Phys. Rev. Lett, 89:215901, (2002).

[75] L.Dougan, S.P.Bates, R.Hargreaves, J.P.Fox, J.Crain, J.L.Finney, V.Réat, and A.K.Soper. Methanol-water solutions: A bi-percolating liquid mixture. J. Chem. Phys, 121:6456-6462, 2004.

[76] J.C.G. Pereira, C.R.A. Catlow, and G.D. Price. Molecular dynamics simulation of liquid h2o, meoh, etoh, si(ome)4, and si(oet)4, as a function of temperature and pressure. J. Phys. Chem. A, 105:1909-1925, 2001.

[77] M. Levitt, M. Hirschberg, R. Sharon, K.E. Laidig, and V. Daggett. Calibration and testing of a water model for simulation of the molecular dynamics of proteins and nucleic acids in solution. J. Phys. Chem. B, 101:5051-5061, 1997.

[78] M. Kumbar and V.T Maddaiah. A conformational study of n-phenyl-1naphthylamine and 1-anilino-8-naphthalene sulfonate by the empirical method. Biochem. Biophys. Acta, 497:707, (1977).

[79] P.Postorino, M.A.Ricci, and A.K.Soper. Water above its boiling point: Study of the temperature and density dependence of the partial pair correlation functions. i. neutron diffraction experiment. J. Chem. Phys, 101:4123-4132, 1994.

[80] B.H. Bransden and C.J. Joachain. Introduction to Quantum Mechanics. Longman Scientific and Technical, UK, (1989).

[81] C.G. Windsor. Pulsed Neutron Scattering. Taylor and Francis, London, UK, 1981. 
[82] L.V. Hove. Correlations in space and time and born approximation scattering in systems if interacting particles. Phys. Rev., 95:249, (1954).

[83] J.E.Enderby and G.W.Neilson(edited by F.Franks). Water: A Comprehensive Treatise. Plenum Press, New York, (1979).

[84] A.K. Soper and A. Luzar. A neutron diffraction study of dimethyl sulphoxidewater mixture. J. Chem. Phys., 97:1320, 1992.

[85] A.K. Soper, W.S. Howells, and A.C. Hannon. Atlas-analysis of time of flight diffraction data from liquid and amorphous samples. RAL Report, RAL, (1989).

[86] M.P. Allen and D.J. Tildesley. Computer Simulation of Liquids. Oxford University Press, UK, (1987).

[87] H. J. C. Berendsen, J. R. Grigera, and T. P. Straatsma. The missing term in effective pair potentials. J. Phys. Chem, 91:6269, 1987.

[88] M. Haughney, M. Ferrario, and I. R. MacDonald. Molecular-dynamics simulation of liquid methanol. J. Phys. Chem, 91:4934, 1987.

[89] D.T. Bowron, A. K. Soper, and J. L. Finney. Temperature dependence of the structure of a 0.06 mole fraction tertiary butanol-water solution. J. Chem. Phys., 114:6203, 2001.

[90] D. Stauffer. Introduction to Percolation Theory. Taylor and Francis, London, (1994).

[91] M. Sahimi. Applications of Percolation Theory. Taylor and Francis, London, (1994).

[92] N. Jan. Large lattice random site percolation. Physica A, 266:72, 1999.

[93] R. E. Gibson. The compressions and specific volumes of aqueous solutions of resorcinoland methanol at $25^{\circ}$ and the behavior of water in these solutions. $J$. Am. Chem. Soc., 57:1551-1557, 1935.

[94] H. Schott. Hydration of primary alcohols. J. Chem. Eng. Data, 14:237-239, 1965.

[95] T.W. Yergovich, G.W. Swift, and F. Kurata. Density and viscosity of aqueous solutions of methanol and acetone from freezing point to $10^{\circ} \mathrm{c}$. J Chem. Eng. Data, 16:222, 1971.

[96] M.L. McGlashan and A.G. Williamson. Isothermal liquid-vapor equilibriums for system methanol-water. J Chem. Eng. Data, 21:196, 1976. 
[97] S.L. Randzio and I. Tomaszkiewicz. Excess enthalpy in the methanol water system at $278.15 \mathrm{k}, 298.15 \mathrm{k}$ and $323.15 \mathrm{k}$ under pressures of $0.1,20$ and 39 mpa. experimental results and their analytical presentation. Thermochimica Acta, 103:281, 1986.

[98] J.L.Finney, D.T.Bowron, R.M.Daniel, P.A.Timmins, and M.A.Roberts. Molecular and mesoscale structures in hydrophobically driven solutions. Biophys Chem, 105:391-398, 2003.

[99] J.-W. Handgraaf, T.S. van Erp, and E.J. Meijer. Ab initio molecular dynamics study of liquid methanol. Chem. Phys. Lett., 367:617-624, 2003.

[100] S.K. Allison, R. Hargreaves, J.P. Fox, and S.P. Bates. Clustering and microimmiscibility in alcohol-water mixtures: Evidence from molecular dynamics simulations. Phys. Rev. B, 71:24201, 2005.

[101] N. Yoshii and S. Okazaki. A large scale and long-time molecular dynamics study of supercritical lennard-jones fluid. an analysis of high temperature clusters. $J$. Chem. Phys., 107:2020, 1997.

[102] A.G. Kalinichev and S.V. Churakov. Thermodynamics and structure of molecular clusters in supercritical water. Fluid Phase Equilibria, 183-184:271, 2001.

[103] D.L. Bergman and A. Laaksonen. Topological and spatial structure in the liquidwater-acetonitrile mixture. Phys. Rev. E, 58:4706, 1998.

[104] A. Oleinikova, I. Brovchenko, A. Geiger, and B. Guillot. Percolation of water in aqueous solution and liquid-liquid immiscibility. J. Chem. Phys., 117:3296, 2002.

[105] I. Brovchenko, A. Geiger, and A. Oleinikova. Clustering of water molecules in aqueous solutions: Effect of water-solute interaction. Phys. Chem. Chem. Phys, 6:1982-1987, 2004.

[106] T. Yamaguchi, K. Hidaka, and A.K. Soper. Erratum: The structure of methanol revisited: a neutron diffraction experiment at $-80^{\circ} \mathrm{c}$ and $+25^{\circ} \mathrm{c}$. Mol. Phys, 97:603, (1999).

[107] G.M. Schneider. Aqueous solutions at pressures up to 2 gpa: Gas-gas equilibria, closed loops, high-pressure immiscibility, salt effects and related phenomena. Phys. Chem. Chem. Phys., 4:845, 2002.

[108] G. Jackson. Theory of closed loop liquid liquid immiscibility in mixtures of molecules with directional attractive forces. Mol. Phys., 72:1365, 1991. 
[109] L.A. Davies, G. Jackson, and L.F. Rull. Simulation study of the link between molecular association and reentrant miscibility for a mixture of molecules with directional interactions. Phys. Rev. Lett., 82:5285, 1999.

[110] K. Marsh and F. Kohler. Thermodynamic properties of associated solutions. $J$. Mol. Liq., 30:13, 1985.

[111] N. T. Skipper. Computer simulation of methane-water solutions. evidence of a temperature-dependent hydrophobic attraction. Chem. Phys. Lett., 270:424, 1993.

[112] G. Hummer, S. Garde, A. E. Garćia, M. Paulaitis, and L. Pratt. The pressure dependence of hydrophobic interactions is consistent withthe observed pressure denaturation of proteins. Proc. Natl. Acad. Sci, 95:1552, 1998.

[113] T. Ghosh, A. E Garćia, and S. Garde. Molecular dynamics simulations of pressure effects on hydrophobic interactions. J. Am. Chem. Soc, 123:10997, 2001.

[114] A. Wallqvist. Pressure dependence of methane solvation in aqueous mixtures and the relation to the structure of liquid water. J. Chem. Phys, 96:1655, 1991.

[115] L.Dougan, R.Hargreaves, S.P.Bates, J.L.Finney, V.Réat, A.K.Soper, and J. Crain. Segregation in aqueous methanol enhanced by cooling and compression. J. Chem. Phys, 122:174514, 2005.

[116] P. L. Privalov and S. J. Gill. Stability of protein structure and hydrophobic interaction. Adv. Protein Chem., 39:191, 1988.

[117] P. L. Privalov and S. J. Gill. The hydrophobic effect-a reappraisal. Pure Applied Chem., 61:1097, 1989.

[118] B. Guillot, Y. Guissani, and S. Bratos. A computer simulation study of hydrophobic hydration of rare gases and of methane. i. themodynamic and structural properties. J. Chem Phys., 95:3643, 1991.

[119] R. L. Mancera and A. D. Buckingham. Temperature effects on the hydrophobic hydration of ethane. J. Phys. Chem., 99:14632, 1995.

[120] J. Hernández Cobos and I. Ortega-Blake. Hydrophobic hydration in methanol aqueous solutions. J. Chem Phys., 103:9261, 1995.

[121] N. T. Skipper, C. H. Bridgeman, A. D. Buckingham, and R. L. Mancera. Computer simulation studies of the hydration and aggregation of simple hydrophobic molecules. Faraday Discussions, 103:141, 1996.

[122] J.N. Murrell and A.D. Jenkins. Properties of Liquids and Solutions, 2nd Edition. Wiley, UK, (1991). 
[123] R.C. Weast. Handbook of Chemistry and Physics, 55th Edition. CRC Press, UK, (1974).

[124] P. Hyncica. Partial molar volumes of organic solutes in water. xii. methanol(aq), ethanol(aq), 1-propanol(aq), and 2-propanol(aq) at t= (298 to 573)k and at pressures up to 30mpa. J. Chem. Thermodynamics, 36:1095, 2004.

[125] M. Sakurai. Densities of dilute solutions of water in benzene and in methanol at $278.15,288.15,298.15,308.15$, and $318.15 \mathrm{k}$. partial molar volumes vw and values of $\mathrm{dvw} / \mathrm{dt}$ for water in benzene and in methanol. J. Chem. Thermodynamics, 14:269, 1982.

[126] A. K. Soper and M. A. Ricci. Structures of high-density and low-density water. Phys. Rev. Lett, 84:2881, 2000. 